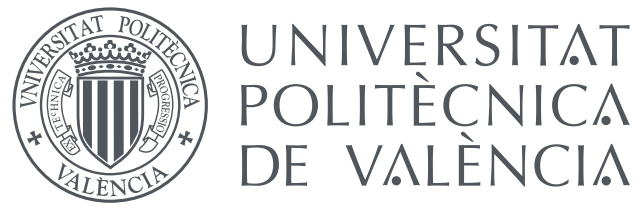

PhD Thesis

\title{
MULTIDISCIPLINARY TECHNIQUES FOR THE SIMULATION OF THE CONTACT BETWEEN THE FOOT AND THE SHOE UPPER IN GAIT: VIRTUAL REALITY, COMPUTATIONAL BIOMECHANICS, AND ARTIFICIAL NEURAL NETWORKS
}

\author{
By \\ María José Rupérez Moreno \\ Supervised by \\ Dr. Carlos Monserrat Aranda \\ Dr. Eugenio Giner Maravilla and \\ Dr. José David Martín Guerrero
}

DEPARTAMENTO DE INGENIERÍA MECÁNICA Y DE MATERIALES

Valencia - July, 2011 

Multidisciplinary Techniques for the Simulation of the Contact between the Foot and the Shoe Upper in Gait: Virtual Reality, Computational Biomechanics, and Artificial Neural Networks.

María José Rupérez Moreno, July 2011 



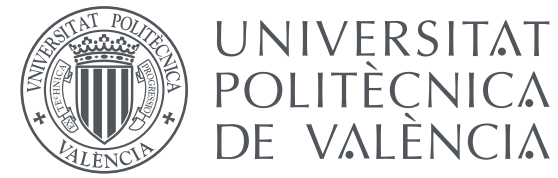

Dpt. de Ingeniería Mecánica y de Materiales.

The undersigned hereby certify that they have read and recommend to the Universidad Politécnica de Valencia for acceptance the thesis entitled Multidisciplinary Techniques for the Simulation of the Contact between the Foot and the Shoe Upper in Gait: Virtual Reality, Computational Biomechanics, and Artificial Neural Networks by María José Rupérez Moreno in partial fulfillment of the requirements for the degree of Doctor of Philosophy. Dated: July, 2011.

Carlos Monserrat Aranda Eugenio Giner Maravilla José D. Martín Guerrero

D. CARLOS MONSERRAT ARANDA, Doctor en Ingeniería Informática, Profesor Titular del Departamento de Sistemas Informáticos y Computación de la Universidad Politécnica de Valencia.

D. EUGENIO GINER MARAVILLA, Doctor en Ingeniería Industrial, Profesor Titular del Departamento de Ingeniería Mecánica y de Materiales de la Universidad Politécnica de Valencia.

D. JOSÉ DAVID MARTÍN GUERRERO, Doctor por la Universidad de Valencia, Profesor Titular del Departamento de Ingeniería Electrónica de la Universidad de Valencia. 

PhD Thesis: MULTIDISCIPLINARY TECHNIQUES FOR THE

SIMULATION OF THE CONTACT BETWEEN

THE FOOT AND THE SHOE UPPER IN GAIT:

VIRTUAL REALITY, COMPUTATIONAL

BIOMECHANICS, AND ARTIFICIAL

NEURAL NETWORKS

Author: MARÍA JOSÉ RUPÉREZ MORENO

Supervisors: Dr. CARLOS MONSERRAT ARANDA

Dr. EUGENIO GINER MARAVILLA

Dr. JOSÉ DAVID MARTÍN GUERRERO

The committee designated to examine the above mentioned doctoral thesis, composed as follows:

President:

Members:

Secretary:

Agrees to grant it the qualification of

Valencia, July 2011 



\section{Acknowledgments}

Me gustaría dar las gracias a todas las personas que de una manera u otra han contribuido a la realización de esta tesis. Especialmente a mis tres directores: Carlos, Eugenio y Jose, por la confianza depositada en mí, su dedicación y su inmensa paciencia conmigo cuando yo la perdía. Espero que estéis orgullosos del trabajo que hemos realizado.

También quería agradecer a Carlos y a Mariano Alcañiz la confianza depositada en mí al permitirme realizar esta tesis en el grupo Labhuman, donde he conocido a gente estupenda durante estos seis años que llevo trabajando en él, y a los cuales también me gustaría dar las gracias. Gracias a los que contribuyeron desde el principio en el desarrollo del motor gráfico: Juan, Eugenio y Oscar, a los que continuaron después: Salva y Moisés, a la persona que me enseñó a optimizar código (o según él a programar): José Miguel, y a los que hacen que día a día mi estancia en el grupo sea lo más agradable posible, las chicas: Patricia, Queca, Sandra, María Teresa y Miriam, y los chicos: Fran, Miguel, Rober, Fer, Juanjo, Juan Antonio y Alejandro. Gracias a mis nuevos compañeros de batalla: Carlos (otra vez) y Valery, y sobre todo, gracias a mis dos grandes amigas: Maja y María Ángeles que han pasado lo bueno y lo malo de esta tesis conmigo.

I would like to thank Christopher Nester the opportunity he gave me to do my stay in Salford where a part of this research was developed. I would also thank people from Thinklab their kind acceptance and their support during the time I spent there. Finalmente, me gustaría dar las gracias a la gente de Inescop que ha colaborado en esta investigación: Enrique, Elena y Pascual, y al resto de grupos que también han colaborado con nosotros en la realización de esta tesis. 

A Jose y a mi familia 

"The worthwhile problems are the ones you can really solve or help solve, the ones you can really contribute something to. ... No problem is too small or too trivial if we can really do something about it."

Richard Feynman. 



\section{Resumen}

Esta Tesis propone el uso de técnicas multidisciplinares como una alternativa viable a los procedimientos actuales de evaluación del calzado los cuales, normalmente, consumen muchos recursos humanos y técnicos. Estas técnicas son Realidad Virtual, Biomecánica Computacional y Redes Neuronales Artificiales. El marco de esta tesis es el análisis virtual del confort mecánico en el calzado, es decir, el análisis de las presiones de confort en el calzado y su principal objetivo es predecir las presiones ejercidas por el zapato sobre la superficie del pie al caminar mediante la simulación del contacto en esta interfaz.

En particular, en esta tesis se ha desarrollado una aplicación software que usa el Método de los Elementos Finitos para simular la deformación del calzado. Se ha desarrollado un modelo preliminar que describe el comportamiento del corte del calzado, se ha implementado un proceso automático para el ajuste pie-zapato y se ha presentado una metodología para obtener una animación genérica del paso de cada individuo. Además, y con el fin de mejorar la aplicación desarrollada, se han propuesto nuevos modelos para simular el comportamiento del corte del calzado al caminar. Por otro lado, las Redes Neuronales Artificiales han sido aplicadas en esta tesis a la predicción de la fuerza ejercida por una esfera, que simulando un hueso, empuja a una muestra de material. Además, también han sido utilizadas para predecir las presiones ejercidas por el corte del calzado sobre la superficie del pie (presiones dorsales) en un paso completo.

Las principales contribuciones de esta tesis son: el desarrollo de un innovador simulador que permitirá a los fabricantes de calzado realizar evaluaciones virtuales de las características de sus diseños sin tener que construir el prototipo real, y el desarrollo de una también innovadora herramienta que les permitirá predecir las presiones dorsales ejercidas por el calzado sobre la superficie del pie al caminar. 



\section{Resum}

Esta Tesi proposa l'ús de tècniques multidisciplinars com a alternativa viable als procediments actuals d'avaluació del confort del calcer els quals, normalment, consumixen molts recursos humans i tècnics. Estes tècniques són Realitat Virtual, Biomecànica Computacional i Xarxes Neuronals Artificials. El marc d'esta tesi es l'avaluació virtual del confort mecànic del calcer, és a dir, de les pressions relacionades amb el confort del calcer; i el seu principal objectiu és predir les pressions exercides per la sabata sobre la superfície del peu al caminar per mitjà de la simulació del contacte en esta interfaç.

En particular, s'ha desenvolupat una aplicació software que utilitza el Mètode dels Elements Finits per tal de simular la deformació del calcer. S'ha desenvolupat un model preliminar que descriu el comportament del tall del calcer, s'ha implementat un procés automàtic per a l'aliniament inicial peu-sabata i s'ha presentat una metodologia per a obtindre una animació genèrica del pas de cada individu. A més, s'han proposat nous models per a simular el comportament del tall del calcer amb l'objectiu de millorar l'aplicació desenvolupada. Per altra banda, les Xarxes Neuronals Artificials (XNA) han sigut aplicades amb èxit a la predicció de la força exercida per una esfera, que simulant un os, espenta una mostra de material. Les XNA també han sigut emprades per tal de predir les pressions exercides pel tall del calcer sobre la superfície del peu (pressions dorsals) en un pas complet.

Les principals contribucions d'esta tesi són, el desenvolupament d'un innovador simulador que permetrà als fabricants de calcer realitzar avaluacions virtuals de les característiques del seus dissenys sense haver de construir el prototip real, i el desenvolupament d'una eina també innovadora que els permetrà predir les pressions dorsals exercides pel calcer al caminar. 



\section{Abstract}

In this thesis, the use of multidisciplinary techniques is proposed as a viable alternative to the current procedures for assessment of footwear, which are costly and time consuming. These techniques are Virtual Reality, Computational Biomechanics, and Artificial Neural Networks. The framework of this research is the virtual assessment of the mechanical comfort in footwear, i.e., comfort pressures in footwear. The main goal of this thesis is to predict the pressures exerted by the shoe over the foot dorsum while walking simulating the contact at this interface. In particular, the Finite Element Method is used to develop a software application for the simulation of the footwear deformation in gait. A first model for the behavior of the shoe upper material is developed. An automatic procedure for the foot-shoe initial alignment is implemented into the application. A methodology to obtain a generic animation of the step for each individual is presented as well as new models for the shoe upper material behavior aimed to improve the developed application. On the other hand, Artificial Neural Networks are successfully applied to predict the force exerted by a sphere that, simulating a bone, pushes a shoe upper material sample. They are also used to predict the pressures exerted by a shoe on the foot dorsum (dorsal pressures) during a complete step.

The main contributions of this thesis are: The development of an innovative simulator that will allow footwear manufacturers to perform virtual evaluations of the functional and esthetic features of their designs without having to construct the real prototype, and the development of another innovative tool for the prediction of the dorsal pressures exerted by the footwear while walking. 



\section{Contents}

1 Introduction 1

1.1 Objectives ...................... 6

1.1.1 Secondary objectives . . . . . . . . . . . . . 6

1.2 Main contributions . . . . . . . . . . . . . . 8

1.3 Outline ............................. 9

2 Background literature $\quad 13$

2.1 Finite Elements in Footwear . . . . . . . . . . . . . . . 13

2.1.1 Two-Dimensional Models . . . . . . . . . . . . 13

2.1.2 Three-Dimensional Models . . . . . . . . . . . 20

2.1.3 Summary of developments in footwear FE models . . . . . 30

2.2 Artificial Neural Networks in Biomechanics . . . . . . . . . . . . . 31

3 Simucal. A footwear simulator for virtual assessment of footwear 37

3.1 Introduction . . . . . . . . . . . . . . . . . . . . . 38

3.2 Methods . . . . . . . . . . . . . . . . . 39

3.2.1 Description of the application . . . . . . . . . . . . 39

3.2.2 Physical model for the shoe upper behavior . . . . . . . . . 41

3.2.3 Description of the simulation . . . . . . . . . . 63

3.3 Results...................... . . 70

3.3.1 Validation of the numerical model . . . . . . . . . 70

3.3.2 Behavior of the materials . . . . . . . . . . 76

3.3.3 Simulation ................... . . 79 


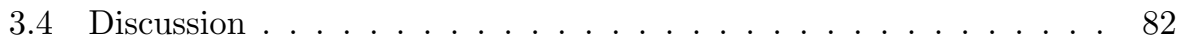

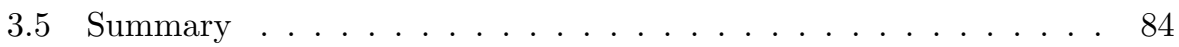

$\begin{array}{lll}4 & \text { Foot animation } & 87\end{array}$

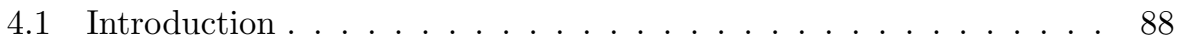

4.2 Obtaining the foot animation $\ldots \ldots \ldots \ldots \ldots$

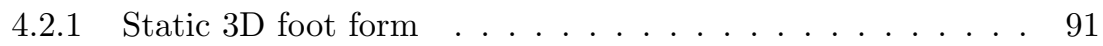

4.2 .2 Foot movement record . . . . . . . . . . . . . . . . 91

4.3 Dorsal Pressures . . . . . . . . . . . . . . . . . . . . . . 93

4.4 Discussion . . . . . . . . . . . . . . . . . . . . . . . . . . 99

4.5 Summary . . . . . . . . . . . . . . . . . . 104

5 Behavior of the shoe upper material in gait 107

5.1 Introduction . . . . . . . . . . . . . . . . . . 108

5.2 Materials and methods . . . . . . . . . . . . . . . . . . . 109

5.2 .1 Uniaxial tensile tests . . . . . . . . . . . . . . . . . . . 113

5.2 .2 Resistance to damage on lasting . . . . . . . . . . . . . 114

5.2.3 Simulation of the shoe forming process using lasts . . . 117

5.3 Results and analysis . . . . . . . . . . . . . . 120

5.3 .1 Uniaxial tensile tests . . . . . . . . . . . . . . 120

5.3 .2 Resistance to damage on lasting . . . . . . . . . . 135

5.3.3 Last forming tests . . . . . . . . . . . . . . . . . 138

5.4 Discussion . . . . . . . . . . . . . . . . . . . . . . . 141

5.5 Summary . . . . . . . . . . . . . . . . . . . . . 145

6 Artificial Neural Networks for modeling flexibility tests $\quad 147$

6.1 Introduction . . . . . . . . . . . . . . . . . . . . 147

6.2 Artificial Neural Networks . . . . . . . . . . . . . . . . . 150

6.2.1 The Multilayer Perceptron . . . . . . . . . . . . . . . . . 152

6.2 .2 Backpropagation algorithm . . . . . . . . . . . . 157

6.3 Materials and methods . . . . . . . . . . . . . . . . 167

6.4 Results . . . . . . . . . . . . . . . . . . . . 172

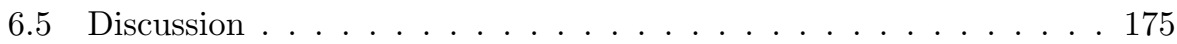




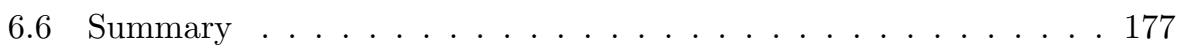

7 Artificial Neural Networks for predicting dorsal pressures over $\begin{array}{lr}\text { the foot surface } & 179\end{array}$

7.1 Introduction . . . . . . . . . . . . . . . . . 180

7.2 Materials and methods . . . . . . . . . . . . . . . 181

7.3 Results of the neural prediction . . . . . . . . . . . . 183

7.4 Discussion . . . . . . . . . . . . . . . . . . 187

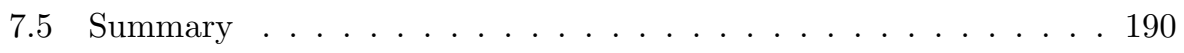

8 Conclusions $\quad \mathbf{1 9 3}$

8.1 Virtual Reality and Finite Element Methods . . . . . . . . . . . . 193

8.2 Artificial Neural Networks . . . . . . . . . . . . . . . . . . 194

8.3 Final conclusions . . . . . . . . . . . . . . 196

8.4 Future work ..................... 197

9 Conclusiones $\quad \mathbf{1 9 9}$

9.1 Realidad Virtual y Elementos Finitos . . . . . . . . . . . . . . . . 199

9.2 Redes Neuronales Artificiales . . . . . . . . . . . . . . . 200

9.3 Conclusiones finales . . . . . . . . . . . . . . . . . 202

9.4 Trabajos futuros .................... 203

Appendix I: The Lin \& Hayhurst model. UMAT subroutine 205

$\begin{array}{lr}\text { Glossary } & 229\end{array}$ 


\section{Chapter 1}

\section{Introduction}

Computer-aided engineering (CAE) has been widely used in the field of engineering to plan, design and evaluate products and manufacturing procedures. The ultimate goal of this computational approach is to provide a systematic and economical tool to ensure the robustness and performance of components and assemblies through the simulation, validation, and optimization procedures. These advances, which are being applied to an increasing number of fields, have also reached footwear design [1].

There is a growing trend among the footwear industries regarding therapeutic and functional footwear that come with various claims of performance and health benefits. Computer-aided design (CAD) approach alone, which focuses mostly on the appearance and dimensions of footwear and its manufacturing is no longer able to fulfill the needs of modern footwear industries. Footwear industry is being modernized by emerging computer technologies, and Cheung et al. [1] assert that a comprehensive CAE system considering both the human musculoskeletal and the footwear structures is expected to take an active role for the evaluation and design of footwear and footwear components. However, according to these authors, 
despite the tremendous potentials for the existing CAE approaches, there is still plenty of rooms for improvement in certain aspects including geometrical, material, contact and loading properties for the foot and the footwear structures before a reliable and accurate CAE system for predicting the foot and footwear biomechanics is possible.

Among the different CAE simulation techniques, the Finite Element (FE) Method is becoming more and more popular because of its versatility and accuracy in modeling irregular geometrical structures, complex material properties, and complicated loading and boundary conditions in both static and dynamic simulations [1]. Footwear researchers started using the FE method in search of more in-depth biomechanical information such as the internal stress and strain distributions of the modeled structures, as the FE method allows for the simulation of the foot-shoe interactions. Most of the simulation processes in footwear have been focused on the interaction between the foot and the shoe sole, even the work flow proposed by Cheung et al., in 2009 [1] for a footwear CAE system only is focused on this part of the shoe (Fig. 1.1). However, few efforts have been made in order to simulate the interaction between the shoe and the foot dorsum (upper surface of the foot), which also influence the comfort [2] and, according to Cheung et al. [1], is necessary for a complete simulation of the footwear performance.

On the other hand, artificial intelligence (AI) raises expectations for advancing $\mathrm{CAD}$ technology. New tools based on knowledge-based systems, fuzzy logic, artificial neural networks and genetic algorithms can enhance the computer-aided design (CAD) systems [3]. These tools lead to intelligent CAD systems (ICAD) and furthermore to intelligent computerintegrated manufacturing systems (ICIM) or intelligent manufacturing systems (IMS) [4]. In this context, AI can also be applied to footwear CAD 


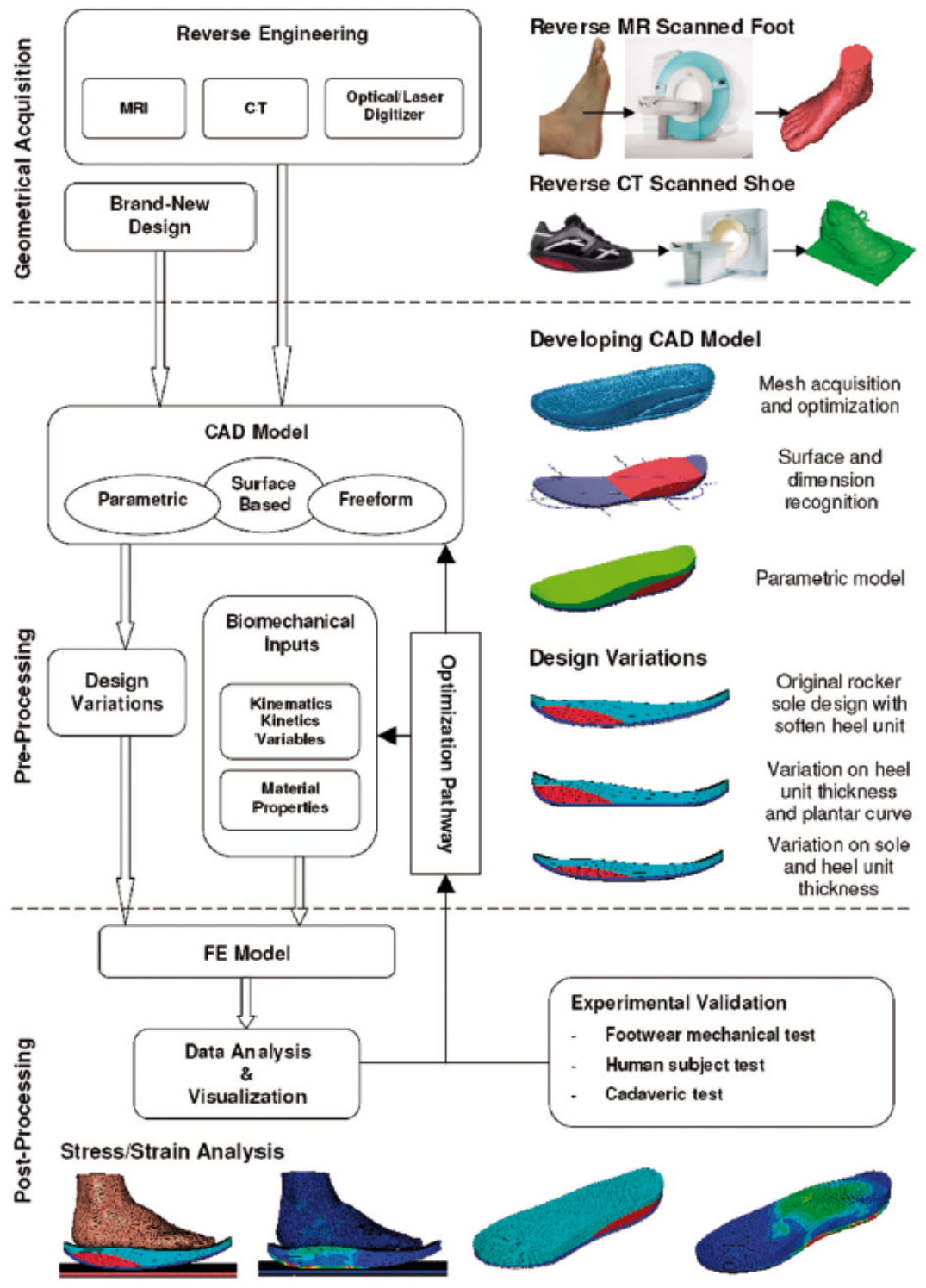

Figure 1.1: Work flow of computer-aided engineering approach for footwear design proposed by Cheung et al. in [1]. 
by introducing these innovative tools in the footwear biomechanics field. Fig. 1.2 shows an example of an ICAD system.

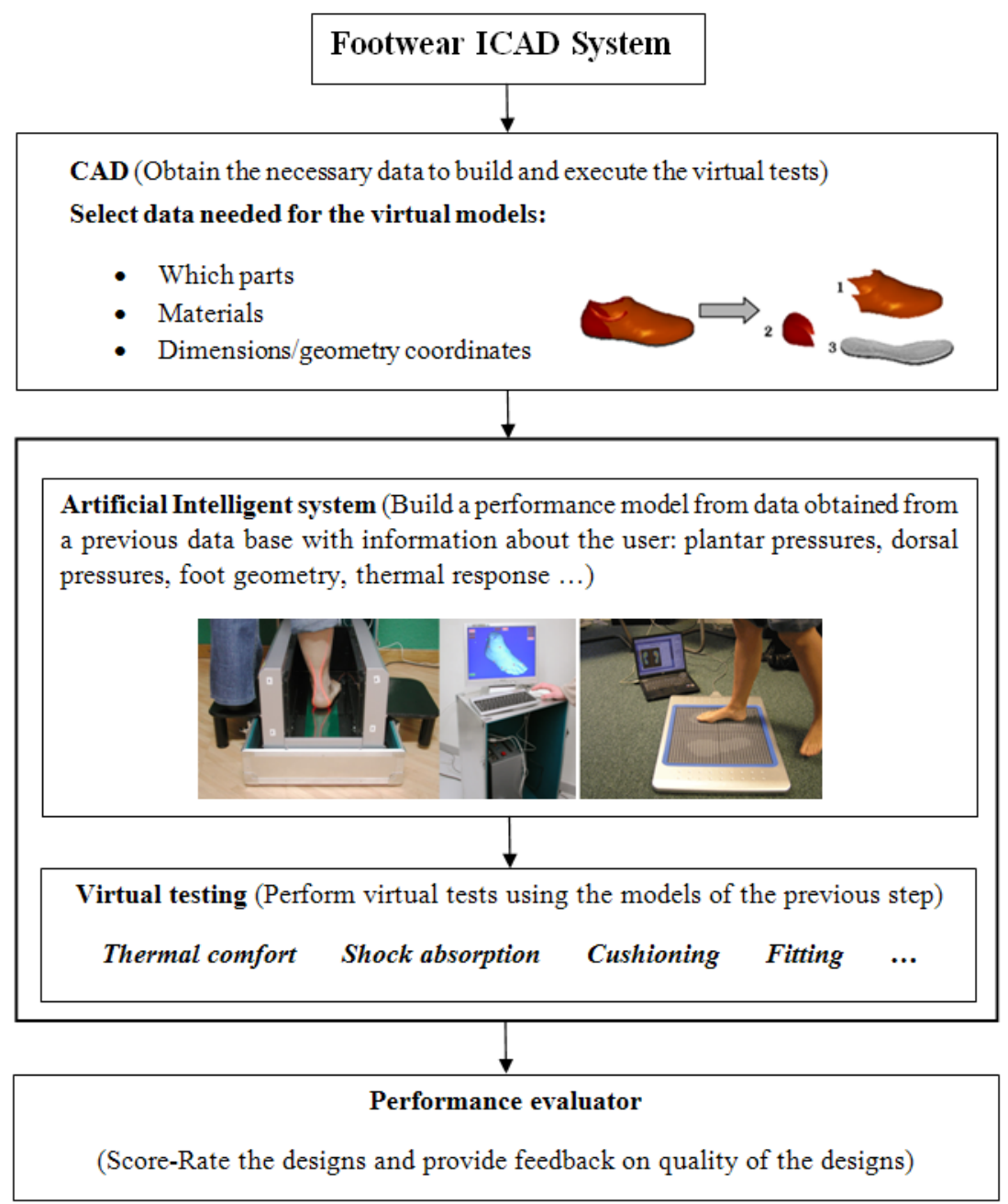

Figure 1.2: Building blocks of the proposed work flow for a footwear ICAD system. 
Among the artificial intelligence techniques, the Artificial Neural Networks (ANNs) are particulary interesting due to their capability of modeling nonlinear behaviors. They are able to perform from a simple classification procedure up to signal processing and data analysis. Therefore, ANNs could become standard methods in Clinical Biomechanics and, even although its use in Biomechanics is still very limited, they are becoming more and more popular [5]. The development of ANNs in Biomechanics has taken place in the analysis of online data; their main applications have been carried out for the classification of people's movements based on several characteristic variables and for biomechanical modeling, where the sequence of input and output variables follows common biomechanical ideas about the movement control, without having deterministic relationships among these variables like EMG (Electromyography)-Force. However, ANNs have never been used to model the interaction between foot and shoe before, thus becoming this research a novel approach in this aspect.

In this thesis, the use of new simulation techniques are proposed as a viable alternative to the procedures currently used for footwear assessment. In particular, the Finite Element Method (FEM) and the Artificial Neuronal Networks (ANNs) are used to simulate the contact between the foot and the shoe upper, and to predict the pressures exerted on these contact areas while walking. The challenge of this thesis is to fill the existing gap about these issues in Footwear Biomechanics. Benefits and drawbacks of both tools, FEM and ANNs, are presented aimed at showing up the difficulties of performing a complete virtual assessment of the footwear performance. 


\section{$1.1 \quad$ Objectives}

The main goal of this thesis is to simulate the interaction between the foot and the shoe upper while walking. For that, different techniques have been applied. These techniques are: Virtual Reality, Computational Biomechanics, and Artificial Neural Networks. The framework of this research is the virtual assessment of the comfort pressures in footwear (mechanical comfort) by means of their prediction during a complete step. The aim of knowing how to predict these pressures is to complete a CAE tool able to perform the entire simulation of the footwear performance.

\subsubsection{Secondary objectives}

The design phase is the most suitable time to predict the mechanical comfort in footwear since performing this prediction from CAD will eliminate the need to make real prototypes to be tested by users, thereby reducing the time and money required to launch new products into the market [6]. With this aim, a software application will be developed, as shown in Chapter 3. This software application will allow a functional analysis of the footwear, assessing the pressures exerted on the foot surface by the shoe. This application will also allow an aesthetic evaluation of the deformation of the footwear.

The next items are required for the software application:

1. A model for the behavior of the footwear.

2. A procedure to simulate the initial alignment between the foot and the shoe.

3. A simulation of the foot movement during a step. 
The research performed in this thesis will aim at achieving these items.

In the development of a model for the footwear behavior, the research will be focused on the shoe upper material. Most of the researchers who have studied the foot-shoe interaction have focused on the shoe sole or insole and their results can be used for this application. Therefore, the first of the secondary objectives of this thesis will be the development of a model to simulate the behavior of the shoe upper materials in gait.

The main problem in the simulation of the gait will arise from the choice of the boundary conditions. Solving this problem will be the second secondary objective of this research.

Determining a procedure for initially aligning the foot to the shoe is a complicated problem since predicting how each individual puts their shoes on is a very difficult task, even the same individual can put different shoes on in a different way. In the application to be developed, the footwear assessment will be based on fitting a shoe to a certain quantity of feet of the same size but with different forms. Therefore, an automation of the process will be necessary, becoming this the third secondary objective of this thesis.

Regarding how the foot movement during a complete step is introduced in the application, a data base of foot animations from distinct users during a complete step will be needed. Developing a procedure to get this data base is the fourth of the secondary objectives of this research.

Finally, alternative techniques to characterize and analyze the foot-shoe upper contact should be studied in search of a more complete study about the prediction of the mechanical comfort in footwear. Alternative tools which can enhance the simulation of the footwear performance and predict the pressures on the foot surface have to be developed. This will be the 
Chapter 1. Introduction

last but not the least of the secondary objectives of this thesis.

\subsection{Main contributions}

The main contribution of this thesis is the use of multidisciplinary techniques to virtually simulate the foot-shoe upper interaction. The aim of this thesis is to fill the existing gap regarding this issue in Footwear Biomechanics. Multidisciplinary approaches are especially appealing here since they combine techniques from different fields, and that is not always an easy task. However, solutions to real problems, like the one tackled in this thesis, usually need to come from multidisciplinary approaches. Therefore, multidisciplinarity becomes a need and a especially valuable characteristic of this thesis.

Three very different techniques have been used in this thesis: Virtual Reality, Computational Biomechanics and Artificial Neural Networks.

- Virtual Reality (VR) together with Computational Biomechanics (CB) have been used to develop an innovative simulator that will allow footwear designers to perform virtual evaluations (in a virtual environment) of the functional and aesthetic features of their designs, without having to construct the real prototype. The developed application presents the subsequent novel advantages:

1. The used virtual feet will be real feet without a standard form whose steps will have been rebuilt in a complete step, so footwear manufacturers will be able to test their design with different forms of same size feet.

2. For the virtual evaluation of the foot-shoe fit, a deformable 
model that simulates the physical behavior of the shoe upper material has been developed. This is novelty of this thesis, necessary to simulate the complete footwear performance [1] since the majority of the biomechanical research related to the footwear has mainly been focused on modeling the shoe sole and insole behavior.

3. The final evaluation of the prototype will not be only static (as in previous reported works $[7,8,9]$ ), but also dynamic because in the future, the manufacturers will be able to perform it during a complete step.

- A totally new approach is presented in this thesis with the use of Artificial Neural Networks (ANNs) to characterize the contact between the foot and the shoe upper. A model to predict the foot-shoe upper contact has been developed aimed at reducing the number of tests needed in the characterization of shoe upper materials. In addition, ANNs have been successfully applied to the prediction of the dorsal pressures over the foot surface. An also innovative tool has been developed to predict these pressures. This predictor presents the advantage that will may be used in the future to predict the pressures on the whole foot surface independently of the kind of shoe used.

\subsection{Outline}

The objectives and main contributions of this thesis are presented in this chapter. Chapter 2 presents the state of the art about the use of FEM and ANNs in footwear aimed at a better understanding of the current framework of this research. 
Chapter 1. Introduction

In Chapter 3, a software application to simulate the deformation of the footwear in gait is presented. The shoe upper is considered to be a homogeneous surface of small thickness that is subjected to bending and plane stress in a 3D space. The study of the shoe upper behavior at small strain zones is considered. A linear elastic model is assumed for the shoe upper. Shell behavior is assigned to the shoe upper, and the Finite Element Method is chosen to solve the problem. Both the Finite Element Method and the solution process are programmed in $\mathrm{C}++$ as an independent module to allow changes of the model that do not affect the rest of the application. In addition, an automation of the initial alignment between the shoe and the foot is presented.

Regarding how the foot movement is introduced into the application, a data base of animations of feet from distinct users during a complete step is needed. The procedure to obtain this data base is presented in Chapter 4. Given that no biomechanical model exists for the foot that simulates its movement during a step due to its complex structure, the animation of the foot is obtained from a foot mesh collected by a scanner in a static position which is deformed using the record of the positions from the main foot anatomical points during a complete step. A study about the viability of obtaining a generic animation in this way must be performed before using this animation in the simulator. This study is carried out analyzing the dorsal pressures (contact pressures) exerted by the shoe upper in gait measured on these same anatomical points also during a complete step.

In order to reduce the assumed hypothesis for the shoe upper behavior in Chapter 3, the behavior of the leather is deeply studied in Chapter 5. The resistance to damage on lasting that some kinds of leathers used as shoe upper materials exhibit is simulated, as well as an experiment specifically designed to simulate a shoe forming process using shoe lasts. 
Both experiments were chosen because they can model the deformation of the shoe upper materials in gait. As the foot surface has little soft tissue, its behavior can be assumed to be determined by the behavior of the bones that form it [10]. In this sense, the resistance to damage on lasting tests, which consist of applying a controlled force by means of a sphere on a sample of the material, could simulate either a bone or a joint pushing the shoe upper. In the case of the simulation of the forming process with lasts, a cylindrical piece made of the same material of the shoe lasts is what pushes the sample of material. Thus, this experiment could model how the dorsum of the foot pushes the shoe upper material while walking. Both tests are simulated using the Finite Element Method. In particular, the commercial software package ANSYS® is used, and the evaluated models are a linear elastic model with an isotropic material behavior, and a linear elastic model with an orthotropic material behavior.

In Chapter 6, alternative techniques to model the foot-shoe upper contact are presented. Artificial Neural Networks (ANNs) are used to get a single model to predict the behavior of the shoe upper material when it is pushed by a sphere simulating a bone (flexibility test). The use of ANNs is proved to be a good procedure to perform this kind of modeling. The chapter shows how a procedure based on Artificial Neural Networks (ANNs) reduces the number of these tests, which can be performed to characterize the materials used as shoe uppers. Using the elastic parameters of the material as inputs to an ANN, a neural model is found which provides a single equation for the relationship between force and displacement instead of a different characteristic curve for each material. The achieved results show the suitability of the novel proposed approach.

In Chapter 7, Artificial Neural Networks (ANNs) are proposed to predict the dorsal pressure over the foot surface exerted by the shoe upper ma- 
terials while walking for four different subjects. A model based on the Multilayer Perceptron (MLP) is used, which provide a single equation to model the exerted pressure for all the materials used as shoe uppers. Five models are produced, one model for each one of the four subjects and an overall model for all the subjects. Due to their good capabilities as nonlinear function approximators, ANNs are proved to be very good predictors of this kind of pressures.

Finally, the conclusions of this thesis are presented in Chapter 8. Benefits and drawbacks of the tools, Virtual Reality, FEM, and ANNs, are benchmarked aimed to better understand the difficulties of performing a complete virtual assessment of the footwear performance. 


\section{Chapter 2}

\section{Background literature}

This chapter presents a review of the background literature related to this thesis. In the following sections, existing FE studies about foot and footwear models are reviewed along with recent FE applications in footwear industry. A review of the most relevant works in which ANNs have made real improvements is also presented, as well as their main applications in Medicine and Biomechanics.

\subsection{Finite Elements in Footwear}

\subsubsection{Two-Dimensional Models}

A great number of FE footwear models have been built for the design and evaluation of orthotic devices, shoe soles and other shoe components. The development and applications of two-dimensional (2D) models for shoe components, orthoses, sport shoes and heeled shoes are presented in the next subsections, together with some works aimed at constructing a $2 \mathrm{D}$ 
full-length model of the foot.

The first known FE analysis of foot and support was reported in 1981 by Nakamura et al. [11], who developed a 2D FE foot model of a unified bony structure of the foot, plantar soft tissue and a shoe sole. A sensitivity analysis on the shoe sole material which varied Young's modulus between 0.08-1000 MPa suggested an optimum range of 0.1-1 MPa for stress reduction in the plantar soft tissue. A further simulation using a nonlinear elastic foamed shoe sole showed similar responses in stress reduction as compared to the predictions with the optimized linearly elastic shoe material.

\section{Orthoses}

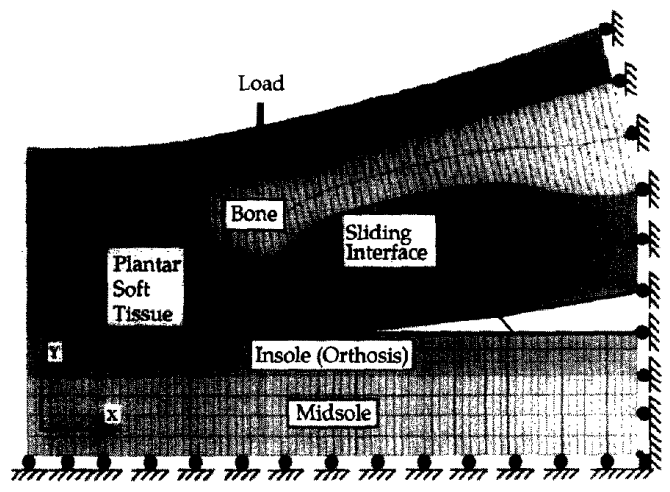

Figure 2.1: The 2D Lemmon's finite element model. Image from [12].

It was not until the late nineties that further developments of foot and footwear became popular. Lemmon el al. in 1997 [12] developed a 2D model for the second metatarsal bone and encapsulated soft tissue to investigate the metatarsal head pressure as a function of six insole thicknesses and two tissue thicknesses. The plantar soft tissue, polyurethane 
insole, and a kind of foamed midsole called by these authors as "Cloud Crepe" were defined as hyperelastic materials. Frictional contact between the foot and support was considered and a vertical load was applied to the metatarsal bone to simulate push off (Fig. 2.1). Orthosis with relatively soft material was found to reduce peak plantar pressure, which also decreased with an increase in insole thickness. The pressure reduction for a given increase of insole thickness was greater when plantar tissue layer was thinner.

A further study performed by this group [13] investigated 36 plug designs of a Microcell Puff midsole including a combination of three materials (Microcell Puff Lite, Plastazote Medium, Poron), six geometries (straight or tapered with different sizes), and two locations of placement (Fig. 2.2). Plugs that were placed according to the most pressurized area were more effective in plantar pressure reduction than those positioned based on the bony prominences. Large plugs (40 mm width) made of Microcell Puff Lite or Plastazote Medium, placed at peak pressure sites, provided the largest peak pressure reductions of up to $28 \%$.

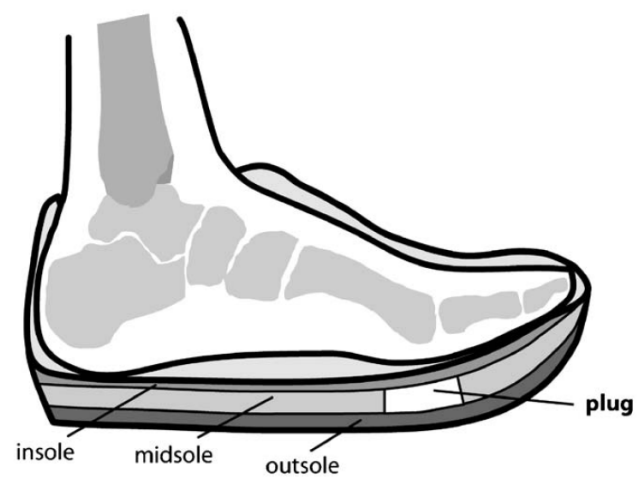

Figure 2.2: A schematic illustration of a plug inserted in the midsole of a shoe. Image from [13]. 


\section{Sport shoes}

Using a similar 2D toe model, Shorten (1998) [14] studied the effect of stud length, surface penetration and sole plate stiffness on induced tissue stress in a soccer shoe sole. Stud length and surface penetration of the stud had only minimal influence on plantar stress distribution. A partially penetrated stud caused only a $5 \%$ increase in peak plantar stress in spite of relatively high stress concentration in the stud and shoe sole. A parametric study on sole plate stiffness suggested that a stiff sole plate is important in protecting tissue above the stud position from high plantar stress with partial stud penetration. The peak plantar stress induced by a rubber sole plate was about $30 \%$ higher than the ordinary nylon plate. Above all, a double-studded condition was able to redistribute more evenly the plantar stress and reduce about $30 \%$ the peak stress.

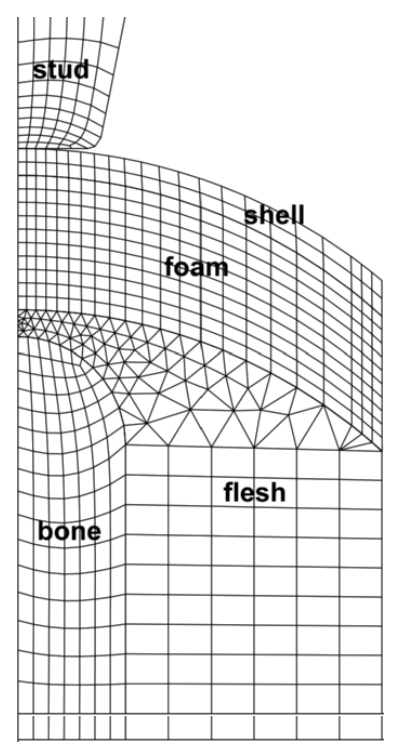

Figure 2.3: Axisymmetric mesh used for FE analysis of stud impacts on protected ankle. Image from [15]. 
In 2004, Ankrah and Milis [15] evaluated the effect of shape and material on the performance of ankle protector against compression of a soccer boot stud using a 2D FE ankle model (Fig. 2.3). By studying the pressure distributions and buckling of different protecting shells, they concluded that thicker foams of higher stiffness should be used instead of thin ethylene vinyl acetate (EVA) foam and low-density polyethylene shell to relieve the ankle from high contact pressure. Weak shell was found to buckle and cause high localized compressive stresses and strains. Shells assigned with Young's modulus of $1 \mathrm{GPa}$ or greater were able to avoid buckling and enable a more evenly distributed stress in the foam and contact pressure at the lateral malleolus.

\section{Heeled shoes}

Several studies used 2D heel models to study the loading response of plantar heel pad. In 2004, Verdejo and Mills [16] developed a 2D hyperelastic FE model of the heel to study the stress distribution in the heel pad during barefoot running and with EVA foamed midsole. The heel pad was found to have a higher order of nonlinearity but a lower initial stiffness than foam material. The predicted peak bare heel plantar pressure was about two times the pressure during shod. Goske et al. in 2006 [17] incorporated a shoe counter (a semi-rigid cup of leather forming the back of the shoe designed to fit around the heel) and a sole into a hyperelastic heel model to investigate the effect of three insole conformity levels (flat, half conforming, full conforming), three insole thickness values (6.3, 9.5, $12.7 \mathrm{~mm}$ ), and three insole materials (Poron Cushioning, Microcel Puff Lite and Microcel Puff) on pressure distribution during heelstrike (Fig. 2.4). Conformity of insole was a more important design factor than insole material in terms of peak pressure reduction. The model predicted a $24 \%$ reduction in peak 
plantar pressure compared to the barefoot condition using flat insoles while the pressure reduced up to $44 \%$ for full conforming insoles. Increasing the insole thickness provided further pressure reduction.

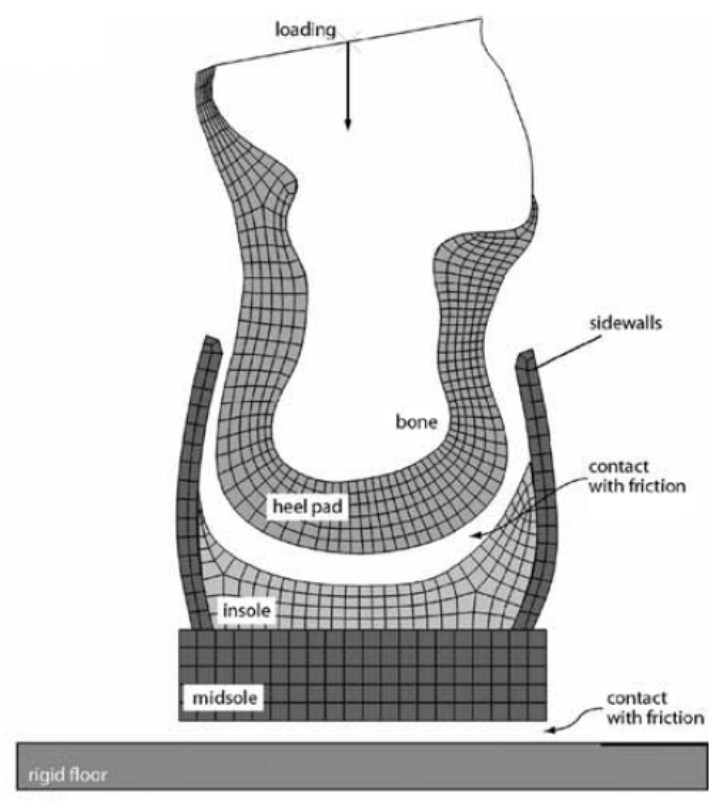

Figure 2.4: Goske's plane strain finite element model. Image from [17].

A similar 2D heel model was developed by Spears et al. in 2007 [18] to study the influence of a heel counter on the stress distribution during standing. Considering a distinction between the material properties of the fat pad and the skin rather than a unified bulk soft tissue provided a better match with the measured barefoot plantar pressure. The predicted stresses in the skin were higher and predominantly tensile in nature, whereas the stress state in the fat pad was hydrostatic. Inclusion of a heel counter to the shod model resulted in an increase in compressive stress of up to $50 \%$ and a reduction in skin tension and shear of up to $34 \%$ and $28 \%$, 
respectively. The compressive and shear stresses in the fat pad reduced up to $40 \%$ and $80 \%$, respectively, while minimal changes were found in tension. A properly fitted heel counter was suggested to be beneficial in terms of heel pad stress relief.

\section{Full-length foot models}

Full-length foot models have been developed recently to evaluate pressurerelieving insole. In 2006, Actis et al. [19] developed six subject-specific 2D FE models of the second and third metatarsal rays from Computer Tomography $(\mathrm{CT})$ images to study the plantar pressure distribution in diabetic feet during push off. The FE model considered unified rearfoot and midfoot structures, one metatarsal and three phalangeal bones connected by cartilaginous structures, flexor tendon, fascia, and encapsulated soft tissue (Fig. 2.5). A total-contact plastazote insert with a rubber shoe sole was incorporated to study its effect on forefoot pressure. Subject-specific bulk soft tissue material properties were obtained and vertical load and moment were applied at the ground support. Frictionless contact interface between the foot-sole and shoe-ground surfaces was assumed. The sensitivity analysis on bone Young's modulus showed a minimal effect on pressure distribution while the corresponding effect of cartilage had a stronger influence. An increase in fascia and flexor tendon Young's modulus produced minimal effects on pressure distribution. Peak metatarsal pressure was reduced by about $46 \%$ with the use of a total-contact insert. The authors suggested that incorporating the bony segments of the rearfoot, metatarsal, and toes, tendon and fascia with linear material properties, and surrounding bulk soft tissue with nonlinear material properties, are plausible simplified configurations for determining metatarsal head pressure distribution during push off. 


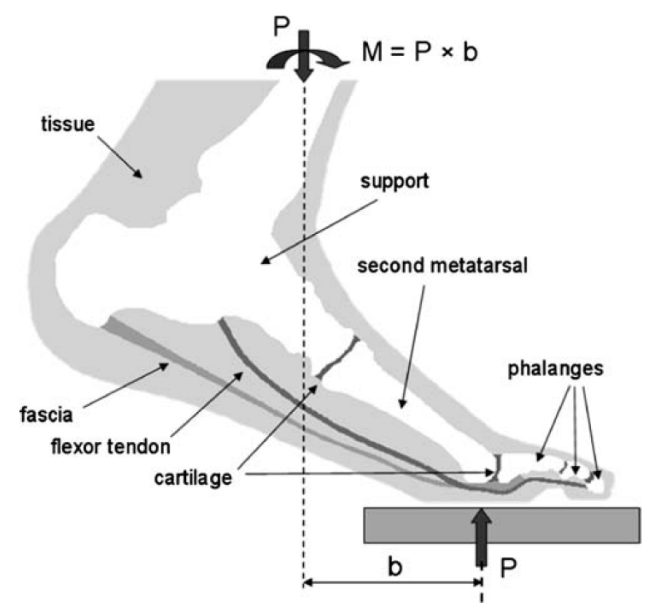

Figure 2.5: Actis' foot model. Image from [19].

A further study performed by Actis et al. in 2008 [20] investigated the effect of Poron multi-plug in a total-contact plastazote insole on forefoot plantar pressure distribution of subjects with either forefoot pain or diabetic neuropathy. Experimentally measured and FE predicted plantar pressure distributions during push off with different total-contact insole configurations (110 plug, multi-cylindrical plugs, box plug and inlay) were examined. Comparing to total-contact insole alone, addition of localized plugs was able to reduce peak plantar pressure up to 14 and $7 \%$ in experiments and FE predictions, respectively.

\subsubsection{Three-Dimensional Models}

A great number of 3D FE models, considering partial, simplified or geometrically detailed foot structures have also been reported in the literature for similar purposes. 


\section{Orthoses}

In 1995, Chu and colleagues [21] developed a linearly elastic model with simplified geometrical features of the foot and ankle to study the loading response of ankle-foot orthosis (Fig. 2.6). During heelstrike and toe off, peak compressive and tensile stresses concentrated at the heel and neck regions of the ankle-foot orthosis, respectively. The highly stressed neck region reflected the common site of orthosis break down. The peak compressive stress in the orthosis increased with increasing Achilles tendon force whereas the peak tensile stress decreased with increasing stiffness of the ankle ligaments. The stress distribution in the orthosis was more sensitive to the stiffness of orthosis than that of the soft tissue.

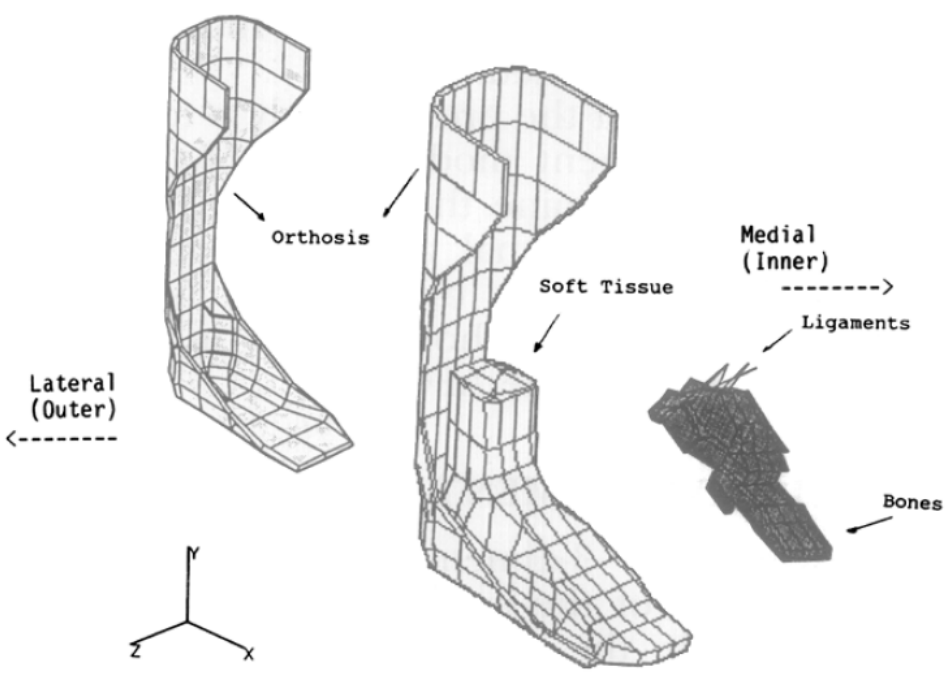

Figure 2.6: Chu's foot, ankle, and orthosis model. Image from [21].

A 3D shell model of plastic ankle foot orthosis considering geometrical and material nonlinearity was developed by Syngellakis et al. in 2000 [22] 
to study its stiffness characteristics. It was found that thickness of ankle foot orthoses had a pronounced effect on the deflection of the ankle-foot orthosis. Material and geometrical nonlinearity were found to be essential in obtaining realistic simulation of the stress distribution of ankle foot orthosis.

\section{Sport shoes}

Several computational models were built to study the performance of varying structural designs for sport surface and shoe sole components. Shorten (1999) [23] used a FE analysis to determine the stress-strain characteristics of hemispherical and hemiellipsoidal spring elements of shoe sole with respect to wall thickness and shape. Inhomogeneous and distinct cushioning characteristics can be configured varying material and geometrical prop-

erties of individual spring element. Baraud et al. (1999) [24] developed hyperelastic FE models of sport surfaces and shoes and demonstrated that an oblique surface-shoe structural configuration could enhance the energy return by more than 14 times that a conventional horizontal shoe-surface system.

\section{Heeled shoes}

Shiang in 1997 [25] introduced a 3D FE model of the shoe heel, composed of insole and midsole with measured heel pressure applied as the loading condition at the heel region. Nonlinear elasticity was defined for the soft tissue and for the insole/midsole properties. The mean peak plantar pressure of the running situation was found to be higher than that of the walking situation as predicted and that the presence of an insole provided better 
cushioning effect. The results showed that nonlinear stress/strain curve and compressibility offered by the nonlinear hyperfoam approach provided a better approximation for the behavior of footwear material because of large deflections of the structures. Addition of an insole layer especially with a contoured surface on top of the midsole was found to provide better cushioning performance for plantar pressure relief.

Even-Tzur et al. in 2006 [26] employed a 3D nonlinear viscoelastic FE model of the shoe-heel interaction to study EVA midsole viscous damping to heel pad stress and strain attenuation during heelstrike in running. Wear of EVA was defined by three different parameters separately: reducing 50 or $90 \%$ the original thickness; increasing the elastic modulus by 25 or $50 \%$; decreasing the relaxation time constants by $50 \%$. The decrease in EVA midsole thickness was found to be the most sensitive factor for heel pad stress, which increased up to $36 \%$. Increasing the elastic modulus and decreasing relaxation time resulted in up to $6 \%$ increase and $3 \%$ decrease of heel pad stress, respectively. The induced heel pad strains were affected by up to $13 \%$ only among all conditions.

\section{Full-length FE sole models}

Several 3D full-length FE sole models were developed for different applications of footwear design and evaluation. Alemany et al. (2003) [27] developed an FE model of a 6-cm high heel shoe sole to optimize shankpiece design (a steel piece used to improve the resistance of high or medium high-heeled shoes) in terms of deformation levels. The orientation angle between the shankpiece and the insole's rear axis, the shankpiece transverse position, and the longitudinal position of a joint that divides the shankpiece into two parts were optimized such that the maximum insole 
deformation was reduced and the gradient smoothed. Sun et al. (2005) [28] developed 3D models of shoe sole from five different sole tread patterns to study their performance of slip resistant on soft soil ground. Traction forces, evaluated by shoe-ground reaction were able to identify the best slip resistant capability among different sole designs. The predicted soil deformations were comparable to the experimental measurements of soil tray test.

A 3D hyperelastic FE model of insole was developed by Barani et al. in 2005 [29] to study the effect of different insole materials including silicon gel, plastazote, polyfoam, and EVA. Discrete pressures were applied at the forefoot and heel regions to simulate the loading on the insole surface during midstance. They concluded from the stress analysis of the insole that most of the materials especially Silicon Gel could be effective in plantar stress reduction. A recent study performed by Nishiwaki (2008) [30] evaluated stiffness distribution of full-length running shoe sole using an FE based on eigen vibration analysis. Three shoe sole designs of different medial and lateral sole reinforcements with l-mm thick hard and soft polyurethane plates of 50 and $20 \mathrm{MPa}$ respectively were modeled. An increase in eigenfrequency of shoe sole during simulated midfoot bending, midfoot torsion and forefoot bending was found to correlate proportionally with decreased sole deformation and propulsion impulse measured experimentally. Shoe sole with relatively stiff reinforcements in both sides produced the highest eigenfrequency and thus least sole deformations in all loading modes. Medial reinforcement was found to be more effective than lateral reinforcement in reducing sole deformation. 


\section{$3 \mathrm{D}$ accurate foot models}

Development of 3D geometrically accurate foot models has become popular in the field of biomechanics since early 2000. A number of researchers have incorporated shoe sole into FE foot model. Oda et al. in 2003 [31] developed a 3D model of foot bones, soft tissue and shoe sole to study the effect of shape and material of a sole plate on the stability and cushioning ability of shoe sole. Heel impact during running was simulated and modified shoe sole resulted in better cushioning from reduced shank acceleration but reduced stability with increased calcaneal pronation.

A 3D linear elastic ankle-foot model, consisting of the bony, encapsulated soft tissue and major plantar ligamentous structures, was developed by Chen et al. in 2003 [32] using CT images to estimate the plantar foot pressure and bone stresses. The joint spaces of the metatarsophalangeal joints and ankle joint were connected with cartilaginous structures while the rest of the bony structures were merged (Fig. 2.7). Frictional contact between the plantar foot and insole support was considered. The efficiency for stress and plantar pressure reduction and redistribution using flat and total-contact insoles with different material combinations was studied (Fig. 2.8). The predicted peak and average normal stresses were reduced in most plantar regions except the midfoot and hallux regions with total-contact insole. The percentage of pressure reduction by totalcontact insole with different combination of material varied with plantar foot regions and the difference was minimal. A further study by the same group in 2007 [33] improved the geometrical details and material models of their previously developed $\mathrm{FE}$ foot model for evaluating pressure-relieving insole. Total-contact insole was again found to reduce significantly the plantar pressure at the forefoot and rearfoot areas.

Cheung and Zhang (2005) [34] developed a linearly elastic FE ankle-foot 

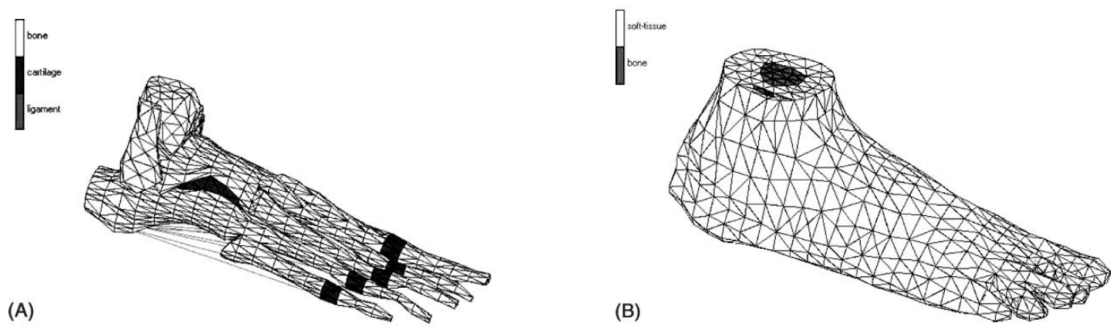

Figure 2.7: (A) Finite element models of the foot bones with cartilage and major plantar ligaments, and $(B)$ the complete model of the foot with soft tissues [32].
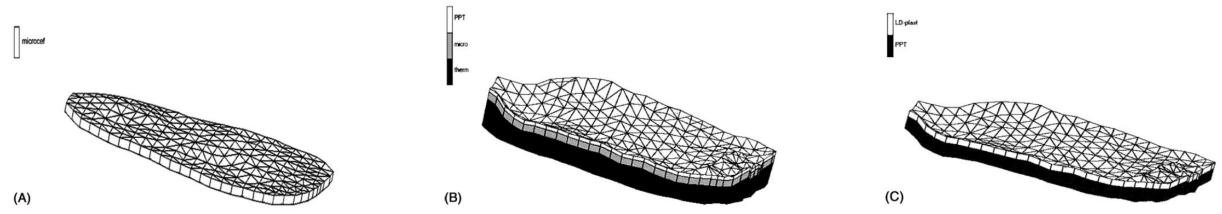

Figure 2.8: (A) Finite element models of the flat insole (consisted of Microcel Puff), (B) TCI-1 (consisted of PPT, Microcel Puff and Thermocork), and (C) TCI-2 (consisted of medium Plastazote and PPT) [32]. 
model from magnetic resonance (MR) images, which took into consideration large deformations and interfacial slip/friction conditions, consisted of 28 bony structures, 72 ligaments and the plantar fascia embedded in a volume of encapsulated soft tissue, to study the interactions between the foot and orthosis (Fig. 2.9). Custom-molded shape was found to be a more important design factor in reducing peak plantar pressure than the stiffness of orthotic material.
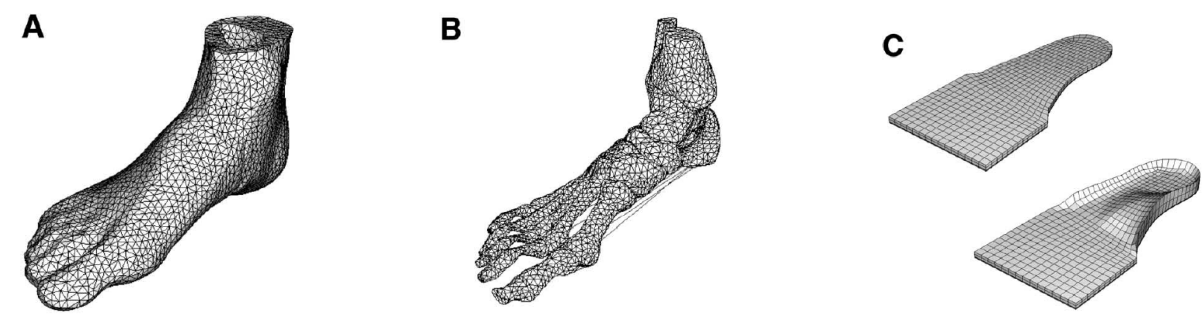

Figure 2.9: (A) The finite element mesh of soft tissues, (B) bony and ligamentous structures, and (C) flat and custom-molded insoles. Figure from [34].

A further study considering the hyperelastic material properties of the encapsulated soft tissue and shoe sole was conducted in 2008 [35] to identify the sensitivity of five design factors (arch type, insole and midsole thickness, insole and midsole stiffness) of foot orthosis assigned with four different levels on peak plantar pressure relief. The Taguchi's experimental design method, which utilizes a fractional factorial design approach to assess the sensitivity of each design factor of a system and to determine its optimal quality level, was used. The custom-molded shape was the most important design factor in reducing peak plantar pressure while the insole stiffness ranked the second most important factor. Other design factors, such as midsole thickness and sole stiffness contributed to less important 
roles in peak pressure reduction in the given order. The FE predictions suggested that custom pressure-relieving foot orthosis providing total-contact fit of the plantar foot is important in the prevention of diabetic foot ulceration. The cushioning insole layer of an orthosis should contribute to the majority of the thickness of the foot orthosis to maximize peak pressure reduction. In the latest development by Cheung et al. [36], the capability of the FE model was extended to 3D FE simulation of foot-shoe interface during walking by incorporating assembled footwear.

Using similar model development techniques, Yu et al. developed in 2008 [37] a female FE model from MR images, which incorporated high-heeled support to investigate the biomechanical effect of heel elevations on foot. Comparing the FE predictions of static standing on flat and high heeled supports, no noticeable rotation movement in transverse plane of the first metatarsophalangeal segment was found. The results implicated that heel elevation was not found to be a direct biomechanical risk factor but might contribute to progressive hallux valgus deformity.

\section{D accurate foot part models}

Several other geometrically accurate FE forefoot, foot and ankle-foot models have been developed recently for the design and evaluation of footwear. Budhabhatti et al. (2007) [38] developed a 3D hyperelastic FE model of the first ray from MR images. The orientation of the model and joint configuration were manipulated to simulate late stance instances from push off to toe off. A flat insole support of five different materials (Puff, Firm Plastazote, Puff Lite, Medium Plastazote, Poron) was modeled to investigate its effect on plantar pressure distribution. The posterior edge of the insole layer was tied to the metatarsal base to simulate the bending 
and movement of the insole with the foot. Among all the insole material, pressure reduction of at least 18 and $43 \%$ could be achieved underneath the metatarsal head and hallux, respectively. Poron insole provided the best pressure reduction by $69 \%$.

Hsu et al. (2008) [39] developed an anatomically detailed linear 3D foot FE model from CT images to investigate the effect of insole contour on the tensile stress in plantar fascia. An optimization procedure was performed with insole shape to identify the optimal insole configuration for plantar fascia stress relief. The values of 15 geometrical references on the insole surface were optimized to improve the conformity of insole to the plantar foot. Comparing to a flat insole, the optimal insole could decrease the plantar fascia stress and peak plantar pressure by 14 and $39 \%$, respectively. Antunes et al. (2008) [40] developed a 3D hyperelastic FE foot model to evaluate the capability of flat insert of cork-polyurethane gel composites for plantar pressure relief during simulated balanced standing. The high contact pressure predicted at the bony prominences was effectively reduced with the use of softer and thicker insoles. Another hyperelastic FE model of foot and assembled shoe was developed by Cui et al. (2008) [41] to study the effect of different sole material inserts, air and water pocket, and reinforcement bars of the sole on impact attenuations. To simulate shoe-ground impact, a mass of $30 \mathrm{~kg}$ and an initial velocity of $1 \mathrm{~m} / \mathrm{s}$ at $30^{\circ}$ normal to the ground were assigned for the foot-shoe model. Soft inserts and water pockets were found to reduce the peak stress and stress concentrations while reinforcement bar facilitated stress propagation to other shoe regions. 


\subsubsection{Summary of developments in footwear FE models}

As it has been described in the previous sections, many 2D and 3D problem have been faced when modeling footwear performance. The interaction of the foot with soles and insoles has been deeply studied, in particular, the interaction with the soft tissues of the foot. The effect of plugs and the influence of bones over these footwear components have also been modeled. Loads on orthoses, on heel shoes, and on different components of sport shoes have been analyzed. In addition, static models of the foot in both 2D and 3D have also been proposed. However, despite all these FE models for soles, insoles, heels, shankpieces, etc., few works about the interaction between the foot and the shoe upper can be found in the literature. Moreover, a model of the foot that simulates its dynamic behavior in gait still remains an unsolved problem.

This thesis is focussed in modeling the interaction of the foot with the shoe upper, and only recently some FE approaches have been applied to evaluate the design and performance of this shoe component.

\section{Shoe upper}

Regarding shoe upper design, Chung et al. (2008) [42] presented a method for planar development of the 3D surfaces of a shoe upper. The 3D surface is first faceted into triangular elements and then roughly laid down on a 2D plane. The nodal points of elements are repositioned by a refinement technique that minimizes the geometric errors. Even after elements have been refined by minimizing geometric errors, the resulting $2 \mathrm{D}$ shape still has some strain energy that needs to be reduced by a relaxation process. These elements are then used as an initial guess for further optimization during which the finite element inverse method is used to minimize the 
total strain energy. Thus, the authors assert that a prototype shoe can be more precisely designed and manufactured.

Regarding the performance, the results of the works presented in Chapter 3 , Chapter 4 and Chapter 5 of this thesis have been recently published in $[6$, 10, 43, 44]. These are the first works that can be found in the literature in which the deformation of the shoe upper is modeled using the FE method. In [43], Rupérez et al. (2008) presented a preliminary computational model to simulate the deformation of the shoe upper materials caused by the foot. The behavior of three different shoe upper materials under linear elastic behavior was simulated. The computer application in which this model runs, Simucal, had previously been presented in [6]. In a further work [10], Rupérez et al. (2010) introduced an automation of the alignment process between foot and shoe. It was also necessary to define a way of obtaining the movements of the foot during a complete step [44] since despite all the FE models developed for the foot, none of them is still able to simulate the dynamic behavior of the foot while walking, but they have been developed essentially to simulate the plantar pressures.

\subsection{Artificial Neural Networks in Biomechanics}

There are two main approaches to describe Artificial Neural Networks (ANNs). Some authors describe ANNs as biological models that can be applied to engineering problems [45]. However, other authors consider ANNs as mathematical models whose way of processing is inspired in some aspects of biological neurons [46, 47, 48]. These two approaches have coexisted in the theory of neural models from the very beginning, so that advances in this theory have come from both approaches. Biological models have provided the inspiration for the development of new artificial neural mod- 
els while mathematical and statistical frameworks have consolidated their practical value [49]. This thesis is focused on the mathematical approach of artificial neural models.

Artificial Neural Networks (in their mathematical approach) have been widely and successfully applied to many fields of knowledge. Basically, they have been applied to problems in which one of the following tasks must be carried out: classification, regression, clustering, or time series prediction. Their application in Medicine, for example, is particulary extensive and relatively common [50]. A reference work in this field can be found in [51], in which the capabilities of ANNs compared to NONMEN (Nonlinear Mixed Effects modeling), a software package used in population pharmacokinetics, were benchmarked. Other works in Medicine can be found in $[52,53,54]$. These work are related with the prediction of the control of the anesthesia. Diabetes is also a deeply studied field of research $[55,56,57]$. Neural networks have also been applied to the assessment of HIV immunopathology [58, 59], and to the optimization of the treatment in patients undergoing chronic renal failure [60, 61].

The development of ANNs in Biomechanics has taken place in a wide range of modalities which can be found in the analysis of online data. Their main applications have been focused on the classification of human movements based on several characteristic variables; and also on biomechanical modeling, in which the sequence of input and output variables follows common biomechanical ideas about movement control, without having deterministic relationships of these variables like EMG(Electromyography)-Force [5].

For the classification of human movement, ANNs have been used for the classification of gait data, the classification of different movement tasks, and for the classification of EMG data. In 1993, Holzreiter \& Köhle [62] classified gait pathologies by means of ground forces. Fast Fourier Trans- 
forms (FFTs) of vertical forces served as inputs to a standard supervised Multilayer Perceptron (MLP). With adequate training of the MLPs, the discrimination of healthy and pathological gait was achieved with an accuracy of up to $95 \%$. In 1997, Bishop et al. [63] investigated several forms of MLP in assigning sensed low back pain during a set of trunk motions to selected kinematic parameters that were collected with a triaxial goniometer. A neural classifier produced superior results with up to $85 \%$ accuracy on test data.

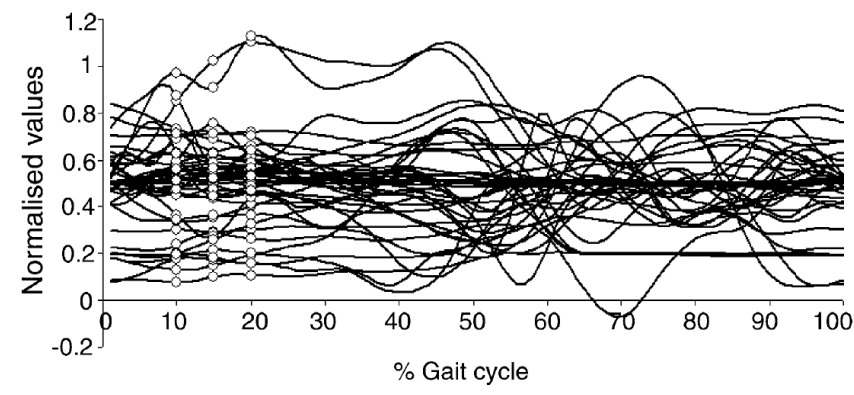

Figure 2.10: A typical graph of all curves of a patient normalized to the gait cycle (horizontal axis) and into the range 0-1 (vertical axis) using the clinical ranges of angles, moments and powers (The curves with values above one or below zero had values off the scale on the conventional plots). Image from [64].

The pilot work of Barton in 1999 [65] visualized the multi-dimensional data space from the gait analysis instrumentation on a self-organizing map (SOM) producing single curves which represented four sagittal plane joint angles. Gait pattern labels were assigned to the SOM by calibrating the map with known data. In a posterior study [66], the findings of physical examination (joint ranges of motion angles) were projected onto a selforganizing topographical map. The arrangement of patients on the map suggested a grouping that was different from the patients' initial classification. However, the groups identified by the SOM matched the classification 

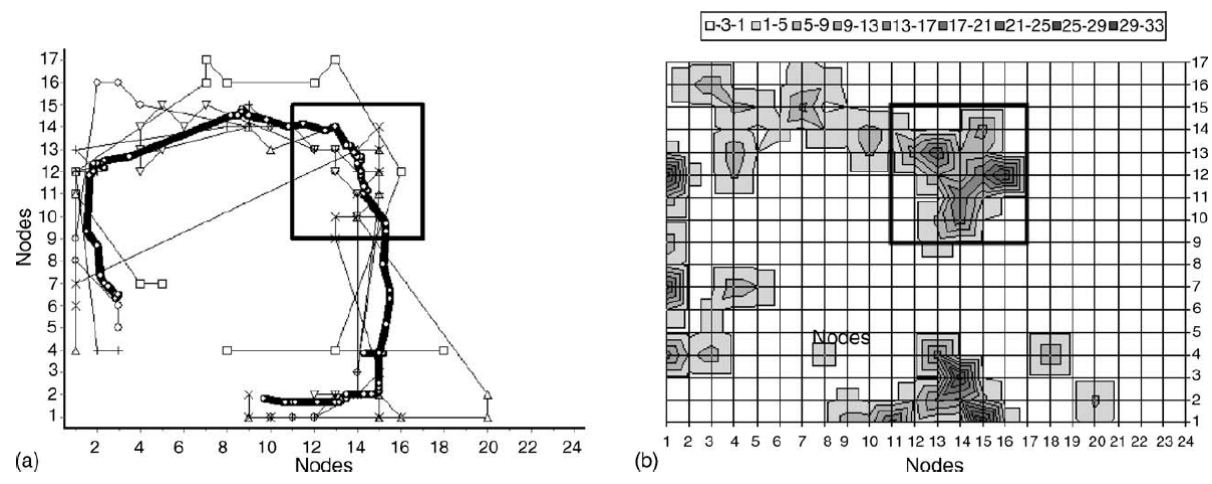

Figure 2.11: (a) Results of six patients whose SOM curves run through a cluster of nodes surrounded by the square. (b) The histogram shows the number of times each node was activated by the six curves. Image from [64].

by gait experts based on full gait analysis. This method was explored in depth in [64] where patients were positioned on the map in relation to each other in order to compare their gait patterns (Fig 2.10 shows a typical graph of all the curves of a patient normalized to the gait cycle and Fig. 2.11 shows how the results of six patients projected onto a SOM improve the analysis of their gait cycle). This method was improved in [67], where the quantization error during the gait cycle was used to quantify the quality of gait.

In the Footwear Biomechanics field, the use of the ANNs is still very limited and few works can be found in the literature. Barton and Lees (1996) [68] compared two insole materials using neural network analysis. Seven male subjects without locomotor disorders walked on a treadmill at a controlled speed and cadence wearing a common shoe and no socks, under three different conditions: two different types of insole of the same thickness and no insole. Pressure-related data from under the foot (within the shoe) were obtained by the MICRO-EMED system during walking. A neural 
network was trained with a back-propagation algorithm to associate sets of pressure-related data with the insole conditions. A neural network analysis was performed to reveal the abstract rules that govern the decision-making processes within the neural network, which were based on the synergistic interactions between the measured variables. The neural network analysis found trends in the way in which the trained neural network responded. The interpretation of those trends provided a limited description of the dynamic behavior of the insoles. It was concluded that neural network analysis could distinguish between insole behavior during use, even though these differences were not significant based on statistical tests. Previously, a work related to the prediction of the friction coefficient was developed in [69]. In this work, ANNs were used as a model to predict the slip resistance with a Normalized Mean Absolute Error (NMAE) of 0.0960 for new inputs once the neuronal model was built. In another work, [70] Kirk et al. (2006) used ANNs to model the traction of studded footwear based on stud input parameters for a single surface. The trained neural networks allowed a rapid prediction of traction forces for any combination of stud variables within the limits of the training data. The networks were trained and tested with further experimental measurements showing an average prediction accuracy of $90 \%$ compared to $64 \%$ when a linear model was implemented.

To the author's knowledge, the most recent works that can be found in the literature about the use of ANNs in Footwear Biomechanics are the works presented in Chapters 7 and 8 of this thesis. In [71], ANNs were for the first time applied to the characterization of the contact between shoe upper and foot surface. In that work, the behavior of three materials under the experimental conditions of simulation of a bone pushing the material is modeled using ANNs. ANNs provided a single model for the behavior of these materials, which can be used for the characterization of 
new materials without having to repeat experiments. In $[72,73]$, ANNs were for the first time used to predict the pressure exerted by five different shoe upper materials over the foot surface for four distinct individuals while walking. A single model is also obtained to predict the dorsal pressures from the material properties and the position of the foot anatomical points on which the pressures are determined.

In summary, practical applications of ANNs have become enormous in the last twenty years. There are applications of ANNs in many other fields different from Medicine and Biomechanics. Finances is a fruitful field for the application of ANNs [74], being especially remarkable the attempts to predict sales and the evolution of the stock market, Industry [75], Marketing [76], Communications [77] or Animal Science [78], only to mention a few of them. Basically, they have been applied to many different practical problems in which tasks of classification, of regression, of clustering, or of time series prediction were carried out. However, although they have been used in gait analysis in Footwear Biomechanics, there is no previous research about the study of the applicability of ANNs for the characterization of the contact between shoe upper and foot surface. Therefore, the current work is novel. 


\section{Chapter 3}

\section{Simucal. A footwear simulator for virtual assessment of footwear}

This chapter presents a software application called Simucal. Simucal is a footwear virtual simulator which was presented by Rupérez et al. in [6] as the first tool to provide footwear manufacturers with the capacity to value the functional features of a design virtually, without having to make real prototypes or use people with feet standard in size and form to try them. The developed application is aimed to minimize the time and the costs that the design of a new shoe collection generates. This application uses a computational model that simulates the deformation of materials in shoe uppers and that quantifies the distribution of forces on the foot surface. A preliminary linear elastic model based on the Finite Element Method (FEM) was developed for the upper. This model can be used for different feet of the same size, for sizes of the same design, and for materials with different elastic parameters [43]. The aim of this application is to provide 
Chapter 3. Simucal. A footwear simulator for virtual assessment of footwear

functional and aesthetic evaluation in CAD footwear design.

\subsection{Introduction}

Comfort is very important in footwear manufacture, because the foot is the structure of the human body that supports most weight in gait. It is also the principal shock absorber of the impact against the ground. Most of the biomechanical research related to the foot has been focused on measuring the plantar pressure distribution, since the presence of high pressures at this zone is one of the major causes of diabetic ulcerations of the foot and pain in the soft tissues $[79,80]$. To measure these pressures, foot models and shoe models have been developed; however the majority of the shoe models that are available only simulate the behavior of the soles and the behavior of the materials they are made of as shown in Chapter 2. To the author's knowledge, there was no models to simulate the deformation of materials in shoe uppers before the development of this application. However, these materials affect comfort [2] and their deformations can be the cause of pain and injury on the foot surface.

The software application presented in this thesis could be an alternative to the subjective procedures currently being used in footwear comfort tests. In these tests, prototypes are made and then evaluated by users with feet standard in size and form [81]. With this computer application, the manufacturer will be able to value functional features such as the fit and the foot-shoe interaction for the different kinds of designed shoes, using only the computer.

The model for the shoe upper developed in this application is based on the Finite Element Method (FEM). Many researchers have pointed out 
that this method has a great capability to model structures with irregular geometry and complex boundary conditions. As the material of the upper can adopt different shapes depending on the design of the shoe and since the boundary conditions change at every stage of the step, this method is regarded as being the most suitable one for providing the most realistic fit possible.

Section 3.2 presents the structure of the standard CAD application developed for the virtual evaluation of footwear. It also describes the model that was developed to simulate the physical behavior of the shoe upper, and explains the process carried out to automatically align the foot to the shoe at the initial stage. Section 3.3 shows the tests that were used to validate the implementation of this model. This validation focuses on evaluating the model and adapting it to the simulation of materials commonly used in footwear manufacture. Finally, Section 3.4 presents a discussion about some aspects of the model and of its application.

\subsection{Methods}

\subsubsection{Description of the application}

The basic structure of the software program Simucal which was developed for the virtual simulation of the footwear performance is shown in Fig. 3.1. Aligner and Simulator are the two basic programs of Simucal. Aligner is the software tool that permits the initial foot-shoe alignment and the decomposition of the shoe into sole and upper (Fig. 3.2). It allows the selection of a physical model, of the boundary conditions, and also the texture of the material. Aligner uses the commercial package 3DStudioMax as the 
base software. Simulator is a program that takes the information generated by the Aligner, the physical model, and the previously established foot movements as input and provides the force diagrams throughout the simulation period.

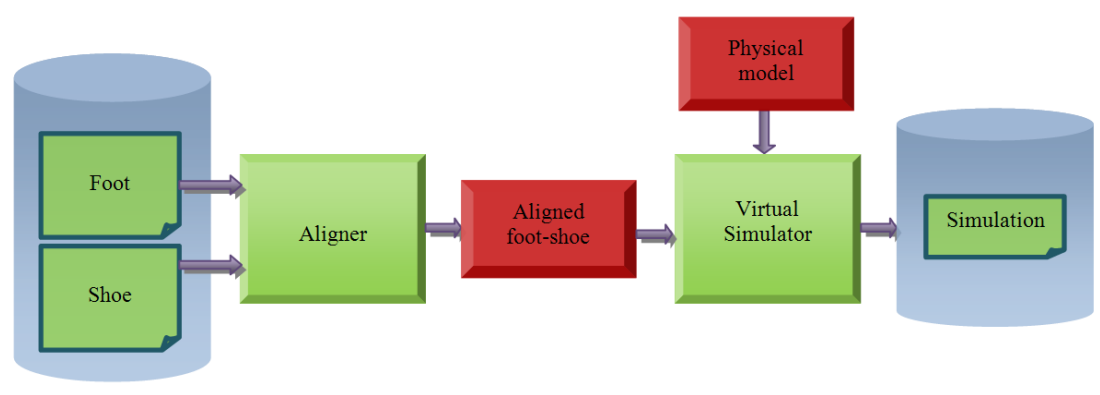

Figure 3.1: Software program structure developed for the simulator.

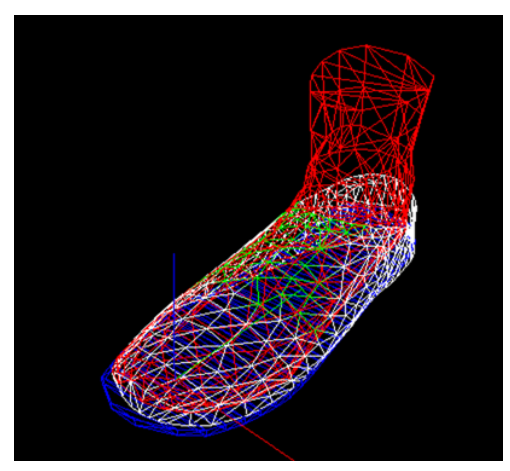

Figure 3.2: Foot, upper and sole meshes.

XSE is a graphical engine developed in language OpenGL by our group LabHuman, a group belonging to the Inter-University Research Institute for Bioengineering and Human Centered Technology (I3BH) of the Universidad Politécnica de Valencia. OpenGL (Open Graphics Library) is a standard specification that defines a cross-language and a cross-platform 
API for writing applications that produce 2D and 3D computer graphics. The interface consists of over 250 different function calls which can be used to draw complex three-dimensional scenes from simple primitives. OpenGL was developed by Silicon Graphics Inc. (SGI) in 1992 and is widely used in $\mathrm{CAD}$, virtual reality, scientific visualization, information visualization, flight simulation, and video games, where it competes with Direct3D on Microsoft Windows platforms. OpenGL is managed by Khronos Group, a non-profit technology consortium. The application Simucal was programmed in $\mathrm{C}++$ as an independent plug-in for XSE, on which the application runs. Simucal was developed as a plug-in for XSE to allow changes in the application that did not affect to the engine. It was also necessary to develop a plug-in for 3DStudioMax able to export files to XSE.

\subsubsection{Physical model for the shoe upper behavior}

A model for the shoe upper behavior was developed using the Finite Element Method (FEM). This method is based on the assumption that the behavior of a continuous body can be represented by the behavior of the body discretizated by elements of smaller size. The FE discretization is performed as follows: The continuous body is divided by lines, areas or volumes in a discrete number of finite elements. The elements are interconnected by a discrete number of points called nodes. Then, the relationship between the position of a point of the continuous body within an element and the position of these nodes is defined by a set of interpolation functions called shape functions. These shape functions are determined by the geometry of the chosen element and must represent the complete characterization of the continuous.

The starting point was the assumption of the upper as an homogeneous 
Chapter 3. Simucal. A footwear simulator for virtual assessment of footwear

surface of small thickness that is subjected to bending and plane stress in a 3D space. In this first version of Simucal, a preliminary linear elastic behavior was assigned to the upper so only three parameters were necessary: Thickness, Young's modulus, and Poisson's ratio. Since the upper is a curved surface of small thickness, the so-called Kirchhoff Thin Shell Theory was applied, and a combination of the three-node triangle for plane stress [82] and the three-node DKT plate element $[83,84]$ was used to make the discretization.

The first model developed for Simucal only simulates the initial moment in which the shoe is fitted to the foot, i.e. when individuals put their shoes on. At this initial stage, the deformations that the shoe suffers can be considered small. Thus, the small strain theory is applied to the problem as a first preliminary approximation to the real behavior of the shoe upper.

In the next subsection, the formulation of the physical model implemented in this thesis for Simucal is presented.

\section{Finite element formulation}

Given an element $e$ defined by its three nodes, the true displacement $\mathbf{u}$ of any point within the element is approximated to a vector $\tilde{\mathbf{u}}$ as in Eq. 3.1, where the components of $\mathbf{N}$ are the shape functions of the suitable element, $\mathbf{a}^{e}$ is the vector of nodal displacements, and $e$ stands for element.

$$
\mathbf{u} \approx \tilde{\mathbf{u}}=\sum_{i=1}^{3} N_{i} a_{i}^{e}
$$


This type of interpolation is repeated for all the elements in the domain. Then, an assembly process is followed and the finite number of unknowns $a_{i}^{e}$ is solved for all the elements. Once all the node displacements are calculated, the strains $\varepsilon$ can be obtained as Eq. 3.2 shows.

$$
\varepsilon=\mathbf{S} \cdot \tilde{\mathbf{u}}
$$

In this equation, $\mathbf{S}$ is an appropriate linear operator of first derivatives that relates the strain with the displacements as Eqs. 3.3 and 3.4 show.

$$
\varepsilon=\mathbf{B}^{e} \cdot \mathbf{a}^{e}
$$

$$
\mathbf{B}^{e}=\mathbf{S} \cdot \mathbf{N}^{e}
$$

Assuming a linear elastic behavior, the relationship between stresses and strains is given by the generalized Hooke law (Eq. 3.5).

$$
\sigma=\mathbf{D}\left(\varepsilon-\varepsilon_{0}\right)+\sigma_{0}
$$

where $\mathbf{D}$ is the material stiffness matrix, $\varepsilon_{0}$ are the initial strains, and $\sigma_{0}$ are the initial stresses. By minimizing the total potential energy, or 
applying the Principle of the Virtual Work, or the Weighted Residuals Method to the linear elastic problem, the algebraic Eq. 3.6 is derived [82].

$$
\mathbf{K}^{e} \cdot \mathbf{a}^{e}=\mathbf{r}^{e}-\mathbf{f}^{e}
$$

In this equation, $\mathbf{K}^{e}$ is the stiffness matrix of the element that can be obtained as shown in Eq. 3.7, $\mathbf{f}^{e}$ is the internal force set (Eq. 3.8), and $\mathbf{r}^{e}$ is the external force set applied at the nodes. The internal forces have been separated in two sets: $\mathbf{f}_{1}^{e}$ and $\mathbf{f}_{2}^{e}$. In $\mathbf{f}_{1}^{e}$ (Eq. 3.9), the first integral are body forces (e.g. forces due to the weight) and the second integral are the forces on the boundary due to surface tractions $\overline{\mathbf{t}}$. In $\mathbf{f}_{2}^{e}$ (Eq. 3.10), the first integral are the forces due to the initial strains and second integral are the forces due to the initial stresses.

$$
\mathbf{K}^{e}=\int_{v} \mathbf{B}^{\mathrm{T}} \mathbf{D B} \mathrm{d} V
$$

$$
\mathbf{f}^{e}=\mathbf{f}_{1}^{e}+\mathbf{f}_{2}^{e}
$$

$$
\mathbf{f}_{1}^{e}=-\int_{v} \mathbf{N}^{\mathrm{T}} \mathbf{b} \mathrm{d} V-\int_{A} \mathbf{N}^{\mathrm{T}} \overline{\mathbf{t}} \mathrm{d} A
$$




$$
\mathbf{f}_{2}^{e}=-\int_{v} \mathbf{B}^{\mathrm{T}} \mathbf{D} \varepsilon_{\mathbf{0}} \mathrm{d} V+\int_{v} \mathbf{B}^{\mathrm{T}} \sigma_{0} \mathrm{~d} V
$$

To obtain the complete solution, a is built containing all the degrees of freedom (DOFs) of all the nodes of the mesh, (Eq. 3.11) and the stiffness matrix is obtained assembling the $\mathbf{K}^{e}$ matrices of the elements as Eq. 3.12 shows. Eq. 3.12 is the system of equations to solve, where $e$ represents the element and takes values from 1 to $m$, the number of elements in the mesh.

$$
\begin{gathered}
\mathbf{a}=\left(\begin{array}{c}
a_{1} \\
\vdots \\
a_{n}
\end{array}\right) \\
\mathbf{r}_{i}=\left(\sum_{e=1}^{m} \mathbf{K}_{i 1}^{e}\right) \cdot \mathbf{a}_{1}+\left(\sum_{e=1}^{m} \mathbf{K}_{i 2}^{e}\right) \cdot \mathbf{a}_{2}+\cdots+\sum_{e=1}^{m} \mathbf{f}_{i}^{e}
\end{gathered}
$$

\section{Stiffness of a plane element in local coordinates}

Let be a plane element simultaneously subjected to two actions of membrane and bending (Fig. 3.3). According to the small strain hypothesis and assuming an isotropic behavior, the Kirchhoff shell theory, which assumes the thickness of the body much smaller than the other dimensions 
and forbids transverse shear deformations, allows decoupling of the bending and the plane stress (membrane) [85]. Given an element located in the $x y$ plane, the strain in the plane is expressed as a function of the displacements along the $x$ and $y$ axis, named $u$ and $v$ respectively, for each node $i$ of the element (Eq. 3.13). The equilibrium equations are obtained minimizing the total potential energy, which provides the nodal forces $\mathbf{f}^{e p}$ as Eq. 3.14 shows, where $e$ stands for element, $p$ for plane stress, and $\mathbf{K}^{e p}$ is the plane stress stiffness matrix of the element.

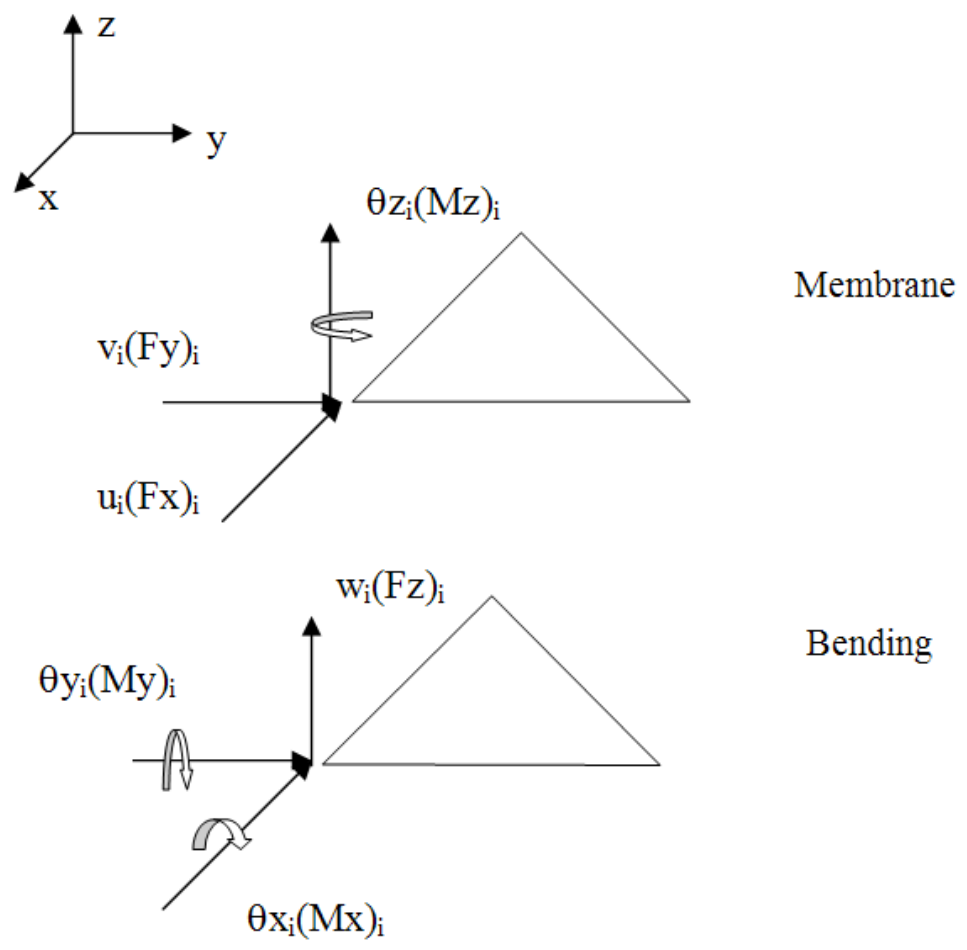

Figure 3.3: Membrane loading and bending on a plane element. 


$$
\mathbf{a}_{i}^{p}=\left(\begin{array}{c}
u_{i} \\
v_{i}
\end{array}\right) ; \mathbf{f}_{i}^{p}=\left(\begin{array}{c}
F_{x_{i}} \\
F_{y_{i}}
\end{array}\right)
$$

$$
\mathbf{f}^{e p}=\mathbf{K}^{e p} \mathbf{a}^{p}
$$

When bending is considered, the strain state is expressed as a function of the displacement along the $z$ axis and the $x$ and $y$ rotations: $w, \theta_{x}$ and $\theta_{y}$ respectively (Eq. 3.15). The nodal forces $\mathbf{f}^{e b}$ are expressed as shown in Eq. 3.16, where $e$ stands for element, $b$ for bending, and $\mathbf{K}^{e b}$ is the bending matrix of the element.

$$
\begin{gathered}
\mathbf{a}_{i}^{b}=\left(\begin{array}{c}
w_{i} \\
\theta_{\mathbf{x}_{\mathbf{i}}} \\
\theta_{\mathbf{y}_{\mathbf{i}}}
\end{array}\right) ; \mathbf{f}_{i}^{b}=\left(\begin{array}{c}
F_{z_{i}} \\
M_{x_{i}} \\
M_{y_{i}}
\end{array}\right) \\
\mathbf{f}^{e b}=\mathbf{K}^{e b} \mathbf{a}^{b}
\end{gathered}
$$

Before combining these stiffnesses, it is important to highlight that under the hypothesis of isotropic (or quasi-isotropic material), the displacements due to the membrane forces do not affect the deformation due to the bending and viceversa. Thus, the total stiffness matrix of an element in local 
coordinates will have the form shown in Eq. 3.17 and the vector of DOFs the form shown in Eq. 3.18.

$$
\begin{aligned}
\mathbf{K}_{r s}^{e} & =\left[\begin{array}{cc}
\mathbf{K}_{r s}^{p} & 0 \\
0 & \mathbf{K}_{r s}^{b}
\end{array}\right] \\
\mathbf{a}_{i} & =\left(\begin{array}{c}
\mathbf{a}_{i}^{p} \\
\mathbf{a}_{i}^{b}
\end{array}\right)
\end{aligned}
$$

The deduced stiffness matrices use a local coordinate system since the membrane and flexion effects are more easily derivable in this system. To assemble the elements and to write the appropriate equations of equilibrium, a transformation from local coordinates to a common global coordinate system will be necessary.

Plane stress stiffness matrix of the element in local coordinates

Let be a triangular element in local axes with its three nodes $i, j, m$ counter-clockwise placed (Fig. 3.4). To calculate the plane stress stiffness matrix of the element, $\mathbf{K}_{r s}^{p}$, the starting point is the easiest interpolation of the displacements i.e., a function of first grade polynomials (Eq. 3.19 and Eq. 3.20) [82].

$$
u=\alpha_{1}+\alpha_{2} x+\alpha_{3} y
$$




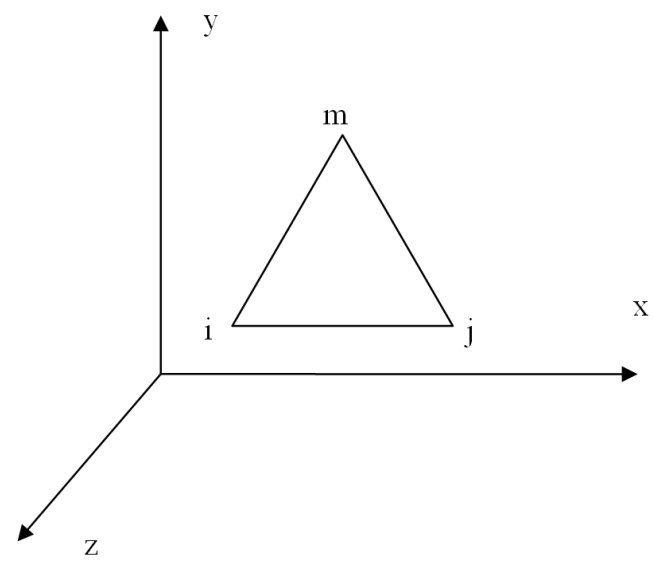

Figure 3.4: Plane triangular element in local coordinates.

$$
v=\alpha_{4}+\alpha_{5} x+\alpha_{6} y
$$

From this representation and using the nodal coordinates, the shape functions are deduced and have the form shown in Eq. 3.21, where $N_{i}$ is the shape function associate with node $i$ and is calculated as Eq. 3.22 shows, where $A$ is the area of the element, and the $a_{i}, b_{i}$, and $c_{i}$ coefficients are given by Eq. 3.23, Eq. 3.24, and Eq. 3.25, respectively.

$$
\begin{gathered}
\mathbf{N}_{i}=N_{i} \mathbf{I}_{2 x 2} \\
N_{i}=\frac{a_{i}+b_{i} x+c_{i} y}{2 A}
\end{gathered}
$$




$$
a_{i}=x_{j} y_{m}-x_{m} y_{j}
$$

$$
b_{i}=y_{j}-y_{m}
$$

$$
c_{i}=x_{m}-x_{j}
$$

$N_{j}$ and $N_{m}$, associated with nodes $j$ and $m$ respectively, are calculated in the same way, with their coefficients $a, b$ and $c$ obtained by cyclic permutations of the subindexes $i, j$ and $m$.

The total strains at any point of the element can be defined by its three components as Eq. 3.26 shows [82].

$$
\varepsilon=\left(\begin{array}{c}
\varepsilon_{x} \\
\varepsilon_{y} \\
\gamma_{x y}
\end{array}\right)=\left[\begin{array}{cc}
\frac{\partial}{\partial x} & 0 \\
0 & \frac{\partial}{\partial y} \\
\frac{\partial}{\partial y} & \frac{\partial}{\partial x}
\end{array}\right] \cdot\left(\begin{array}{l}
u \\
v
\end{array}\right)=\mathbf{S u}
$$

On the other hand, using the FE interpolation, $\varepsilon$ can also be written as Eq. 3.27 shows. 


$$
\varepsilon=\mathbf{B a}^{\mathbf{e}}=\left[\begin{array}{lll}
\mathbf{B}_{i} & \mathbf{B}_{j} & \mathbf{B}_{m}
\end{array}\right]\left(\begin{array}{c}
\mathbf{a}_{i} \\
\mathbf{a}_{j} \\
\mathbf{a}_{m}
\end{array}\right)
$$

Being $\mathbf{B}_{i}$, the matrix given by Eq. 3.28 associated with node i. The same procedure is followed to obtain $\mathbf{B}_{j}$ and $\mathbf{B}_{m}$. Thus, $\mathbf{B}$ matrix is explicitly defined.

$$
\mathbf{B}_{i}=\mathbf{S N}_{i}=\left[\begin{array}{cc}
\frac{\partial \mathbf{N}_{i}}{\partial x} & 0 \\
0 & \frac{\partial \mathbf{N}_{i}}{\partial y} \\
\frac{\partial \mathbf{N}_{i}}{\partial y} & \frac{\partial \mathbf{N}_{i}}{\partial x}
\end{array}\right]=\frac{1}{2 A}\left[\begin{array}{cc}
b_{i} & 0 \\
0 & c_{i} \\
c_{i} & b_{i}
\end{array}\right]
$$

Once the $\mathbf{B}$ matrices are calculated, the stiffness matrix of the element can be obtained using Eq. 3.7. As it was pointed out before, a preliminary linear elastic behavior was assigned to the upper so only three parameters were necessary, the thickness, the Young's modulus, and the Poisson's ratio. In our case, a constant thickness is assumed for the upper and due to the assumption of the upper as homogenous, $\mathbf{D}$ matrix is also constant [82]. Taking into account that $\mathbf{B}$ is constant too since $\mathbf{B}$ matrix is independent of the position of the point within the element for the linear interpolation used here (Eq. 3.28), Eq. 3.7 leads to Eq. 3.29, where $t$ stands for the thickness and $A$ for the area of the element. Thus, the plane stress stiffness matrix is totally defined by this equation where the stiffness matrix of the material D, is given by Eq. 3.30 [82], where $E$ represents the Young's modulus and 
$\nu$ the Poisson's coefficient.

$$
\begin{gathered}
\mathbf{K}_{r s}^{p}=t \cdot A \cdot \mathbf{B}_{r}^{T} \mathbf{D B}_{s} \\
\mathbf{D}=\frac{E}{1-\nu^{2}}\left(\begin{array}{ccc}
1 & \nu & 0 \\
\nu & 1 & 0 \\
0 & 0 & \frac{1-\nu}{2}
\end{array}\right)
\end{gathered}
$$

\section{Bending stiffness matrix of the DKT element in local coordinates}

For the calculation of the bending stiffness of the element, the plate theory establishes that the displacements are given as in Eq. 3.31 [85]. In the shell approximation, $z$ tends to zero, thus $u$ and $v$ displacements are independent of the bending [85].

$$
u=-\theta_{x} \cdot z ; \quad v=-\theta_{y} \cdot z ; \quad w=w(x, y)
$$

When bending is considered, the deformation state is univocally determined by the displacement of the nodes in $z$ direction, and the rotations $\theta_{\mathbf{x}}$ and $\theta_{\mathbf{y}}$ (Eq. 3.32). The $w$ representation is a four grade polynomial, and the rotations are given by Eq. 3.33 and Eq. 3.34 [85]. 


$$
\mathbf{a}_{i}=\left(\begin{array}{c}
w_{i} \\
\theta_{\mathbf{x}_{\mathbf{i}}} \\
\theta_{\mathbf{y}_{\mathbf{i}}}
\end{array}\right)
$$

$$
\theta_{x i}=-\frac{\partial w_{i}}{\partial y}
$$

$$
\theta_{y i}=\frac{\partial w_{i}}{\partial x}
$$

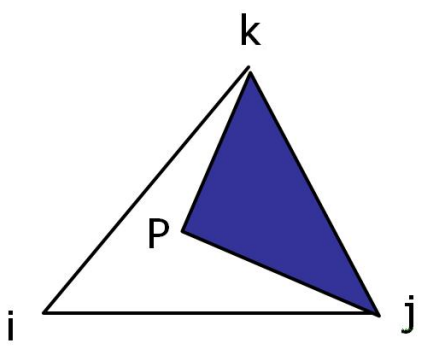

$$
L_{i}=\frac{\operatorname{area}[P j k]}{\text { area }[i j k]}
$$

Figure 3.5: Area coordinates.

The chosen element has three nodes and it is referred to as DKT (Discrete Kirchhoff Triangular) element $[83,84]$. Defining the shape functions of this 
element is necessary to calculate the bending stiffness matrix. The shape functions of this element are functions of the area coordinates $L_{1}, L_{2}, L_{3}$. The area coordinates of a point $P$ that belongs to a triangle are the relative areas of the triangles defined by $P$ and the sides of the principal triangle (Fig. 3.5).

For the DKT element, the shape functions have the form shown in Eq. 3.35.

$$
\left(\begin{array}{c}
f_{1} \\
f_{2} \\
f_{3} \\
f_{4} \\
f_{5} \\
f_{6}
\end{array}\right)=\left(\begin{array}{c}
L_{1}\left(2 L_{1}-1\right) \\
L_{2}\left(2 L_{2}-1\right) \\
L_{3}\left(2 L_{3}-1\right) \\
4 L_{2} L_{3} \\
4 L_{3} L_{1} \\
4 L_{1} L_{2}
\end{array}\right)
$$

The shape functions allow to construct the $\mathbf{H}$ matrices used to calculate the $B$ matrices (Eqs. 3.36 and 3.37).

$$
\mathbf{H}_{x}\left(L_{2}, L_{3}\right)=\left[\begin{array}{c}
1.5\left(a_{6} f_{6}-a_{5} f_{5}\right) \\
b_{5} f_{5}+b_{6} f_{6} \\
f_{1}-c_{5} f_{5}-c_{6} f_{6} \\
1.5\left(a_{4} f_{4}-a_{6} f_{6}\right) \\
b_{4} f_{4}+b_{6} f_{6} \\
f_{2}-c_{4} f_{4}-c_{6} f_{6} \\
1.5\left(a_{5} f_{5}-a_{4} f_{4}\right) \\
b_{4} f_{4}+b_{5} f_{5} \\
f_{3}-c_{5} f_{5}-c_{4} f_{4}
\end{array}\right]
$$




$$
\mathbf{H}_{y}\left(L_{2}, L_{3}\right)=\left[\begin{array}{c}
1.5\left(d_{6} f_{6}-d_{5} f_{5}\right) \\
-f_{1}+e_{5} f_{5}+e_{6} f_{6} \\
-\left(b_{5} f_{5}+b_{6} f_{6}\right) \\
1.5\left(d_{4} f_{4}-d_{6} f_{6}\right) \\
-f_{2}+e_{4} f_{4}+e_{6} f_{6} \\
-\left(b_{4} f_{4}+b_{6} f_{6}\right) \\
1.5\left(d_{5} f_{5}-d_{4} f_{4}\right) \\
-\left(f_{3}+e_{5} f_{5}+e_{4} f_{4}\right) \\
-\left(b_{4} f_{4}+b_{5} f_{5}\right)
\end{array}\right]
$$

where $a_{k}, b_{k}, c_{k}, d_{k}$ and $e_{k}$ are given in Eqs. 3.38 to 3.42, for $k=4,5,6$; and for $i j=23,31,12$.

$$
\begin{gathered}
a_{k}=-\frac{x_{i j}}{l_{i j}^{2}} \\
b_{k}=\frac{3 x_{i j} y_{i j}}{4 l_{i j}^{2}} \\
c_{k}=\frac{\left(\frac{1}{4} x_{i j}^{2}-\frac{1}{2} y_{i j}^{2}\right)}{l_{i j}^{2}}
\end{gathered}
$$




$$
\begin{gathered}
d_{k}=-\frac{y_{i j}}{l_{i j}^{2}} \\
e_{k}=\frac{\left(\frac{1}{4} y_{i j}^{2}-\frac{1}{2} x_{i j}^{2}\right)}{l_{i j}^{2}} \\
l_{i j}^{2}=x_{i j}^{2}+y_{i j}^{2}
\end{gathered}
$$

Finally, B matrix is obtained as Eq. 3.44 shows.

$$
\mathbf{B}\left(L_{2}, L_{3}\right)=\frac{1}{2 A}\left[\begin{array}{c}
y_{31} \frac{\partial \mathbf{H}_{x}^{\mathrm{T}}}{\partial L_{2}}+y_{12} \frac{\partial \mathbf{H}_{x}^{\mathrm{T}}}{\partial L_{3}} \\
-x_{31} \frac{\partial \mathbf{H}_{y}^{\mathrm{T}}}{\partial L_{2}}-x_{12} \frac{\partial \mathbf{H}_{y}^{\mathrm{T}}}{\partial L_{3}} \\
-x_{31} \frac{\partial \mathbf{H}_{x}^{\mathrm{T}}}{\partial L_{2}}-x_{12} \frac{\partial \mathbf{H}_{x}^{\mathrm{T}}}{\partial L_{3}}+y_{31} \frac{\partial \mathbf{H}_{y}^{\mathrm{T}}}{\partial L_{2}}+y_{12} \frac{\partial \mathbf{H}_{y}^{\mathrm{T}}}{\partial L_{3}}
\end{array}\right]
$$

The material stiffness matrix for bending is given as shown Eq. 3.45 in $[85]$. 


$$
\mathbf{D}_{b}=\frac{E t^{3}}{12\left(1-\nu^{2}\right)}\left(\begin{array}{ccc}
1 & \nu & 0 \\
\nu & 1 & 0 \\
0 & 0 & \frac{1-\nu}{2}
\end{array}\right)
$$

Then, the stiffness matrix of the element DKT is calculated as Eq. 3.46 shows.

$$
\begin{gathered}
\mathbf{K}_{\mathrm{DKT}}^{e}=2 A \int_{0}^{1} \int_{0}^{1-L_{3}} \mathbf{B}^{\mathrm{T}} \mathbf{D}_{b} \mathbf{B d} L_{2} \mathrm{~d} L_{3} \\
\mathbf{K}_{\mathrm{DKT}}=\left(\begin{array}{lll}
\left(\mathbf{K}_{11}\right)_{3 \times 3} & \left(\mathbf{K}_{12}\right)_{3 \times 3} & \left(\mathbf{K}_{13}\right)_{3 \times 3} \\
\left(\mathbf{K}_{21}\right)_{3 \times 3} & \left(\mathbf{K}_{22}\right)_{3 \times 3} & \left(\mathbf{K}_{23}\right)_{3 \times 3} \\
\left(\mathbf{K}_{31}\right)_{3 \times 3} & \left(\mathbf{K}_{32}\right)_{3 \times 3} & \left(\mathbf{K}_{33}\right)_{3 \times 3}
\end{array}\right)
\end{gathered}
$$

$\mathbf{K}_{\mathrm{DKT}}^{e}$ is represented as Eq. 3.47 shows, where each element corresponds to a matrix $\mathbf{K}_{r s}^{b}$.

\section{Total stiffness matrix of the element in local coordinates}

The stiffness matrix of the combination of the three-node triangle of the plane stress (two DOFs per node) [82] and the three-node DKT plate element (three DOFs per node) $[83,84]$ is obtained as shown in Eq. 3.48. 


$$
\mathbf{K}^{e}=\left[\begin{array}{cccccc}
\left(\mathbf{K}_{11}^{p}\right)_{2 \times 2} & (0)_{2 \times 3} & \mathbf{K}_{12}^{p} & 0 & \mathbf{K}_{13}^{p} & 0 \\
(0)_{3 \times 2} & \left(\mathbf{K}_{11}^{b}\right)_{3 \times 3} & 0 & \mathbf{K}_{12}^{b} & 0 & \mathbf{K}_{13}^{b} \\
\mathbf{K}_{21}^{p} & 0 & \mathbf{K}_{22}^{p} & 0 & \mathbf{K}_{23}^{p} & 0 \\
0 & \mathbf{K}_{21}^{b} & 0 & \mathbf{K}_{22}^{b} & 0 & \mathbf{K}_{23}^{b} \\
\mathbf{K}_{31}^{p} & 0 & \mathbf{K}_{32}^{p} & 0 & \mathbf{K}_{33}^{p} & 0 \\
0 & \mathbf{K}_{31}^{b} & 0 & \mathbf{K}_{32}^{b} & 0 & \mathbf{K}_{33}^{b}
\end{array}\right]_{15 \times 15}
$$

\section{Coordinate transformation}

As it was pointed out before, to assemble the elements and to write the appropriate equations of equilibrium, a transformation from the local coordinate system to a common global coordinate system is needed. In addition, due to the possibility that the $\theta_{x}$ and $\theta_{y}$ rotations provide components over the $z$ axis during this transformation, the $\theta_{z}$ rotation must be introduced in the formulation of the problem. The same situation occurs with the transformation of bending moments, which requires the third nodal moment $M_{z}$ to be also introduced. Therefore, even though the $z$ rotation is not a degree of freedom in the definition of the strains, it must be introduced in the model along with its associated stiffness [86]. Thus, the submatrix of the element stiffness matrix associated with each node is written as shown in Eq. 3.49 and the vector of DOFs as shown in Eq. 3.50.

$$
\mathbf{K}_{r s}=\left(\begin{array}{ccc}
\mathbf{K}_{r s}^{p} & 0 & 0 \\
0 & \mathbf{K}_{r s}^{b} & 0 \\
0 & 0 & \mathbf{M}_{\theta_{z}}
\end{array}\right)
$$




$$
\mathbf{a}_{i}=\left(\begin{array}{c}
\mathbf{a}_{i}^{p} \\
\mathbf{a}_{i}^{b} \\
\theta_{z_{i}}
\end{array}\right)
$$

The relationship between the local and global components of the DOFs and loads are given by Eq. 3.51 and Eq. 3.52 respectively.

$$
\begin{gathered}
\mathbf{a}_{i}^{\prime e}=\mathbf{L}_{i}^{e} \mathbf{a}_{i}^{e} \\
\mathbf{f}_{i}^{\prime e}=\mathbf{L}_{i}^{e} \mathbf{f}_{i}^{e}
\end{gathered}
$$

where $a_{i}^{e}$ and $f_{i}^{e}$ are the DOF and force vectors in global axes for a node, in which the $z$ rotation and $M_{z}$ moment have been introduced (Eq. 3.53, Eq. 3.54).

$$
\mathbf{a}_{i}^{e}=\left[u_{i}, v_{i}, w_{i}, \theta_{x i}, \theta_{y i}, \theta_{z i}\right]^{\mathrm{T}}
$$




$$
\mathbf{f}_{i}^{e}=\left[f_{x i}, f_{y i}, f_{z i}, M_{x i}, M_{y i}, M_{z i}\right]^{\mathrm{T}}
$$

$\mathbf{L}_{i}^{e}$ is the transformation matrix of nodal displacements and forces from $x^{\prime}$, $y^{\prime}, z^{\prime}$ local axes to $x, y, z$ global axes, which will be constant because the chosen element is plane [85]. From the vector transformation rules, $\mathbf{L}_{i}^{e}$ is deduced (Eq. 3.55). In this equation, $\lambda_{i}^{e}$ is given by Eq. 3.56, where $\lambda_{x^{\prime} x}$ is the cosine of the angle that the axis $x$ forms with the axis $x^{\prime}$, etc. $\tilde{\lambda}_{i}^{e}$ is the rotation transformation and it is given by equation Eq. 3.57.

$$
\begin{aligned}
& \mathbf{L}_{i}^{e}=\left[\begin{array}{cc}
\left(\lambda_{i}^{e}\right)_{3 \times 3} & 0 \\
0 & \left(\tilde{\lambda}_{i}^{e}\right)_{2 \times 3}
\end{array}\right] \\
& \lambda_{i}^{e}=\left[\begin{array}{ccc}
\lambda_{x^{\prime} x} & \lambda_{x^{\prime} y} & \lambda_{x^{\prime} z} \\
\lambda_{y^{\prime} x} & \lambda_{y^{\prime} y} & \lambda_{y^{\prime} z} \\
\lambda_{z^{\prime} x} & \lambda_{y^{\prime} z} & \lambda_{z^{\prime} z}
\end{array}\right]_{i}^{e} \\
& \tilde{\lambda}_{i}^{e}=\left[\begin{array}{lll}
\lambda_{x^{\prime} x} & \lambda_{x^{\prime} y} & \lambda_{x^{\prime} z} \\
\lambda_{y^{\prime} x} & \lambda_{y^{\prime} y} & \lambda_{y^{\prime} z}
\end{array}\right]_{i}^{e}
\end{aligned}
$$

A matrix $\mathbf{T}_{i}^{e}$ can be defined, Eq. 3.58, where $n$ is the number of nodes of the element. This matrix allows the transformation of the DOF and the force vectors as shown in Eq. 3.59 and Eq. 3.60 respectively. Thus, the 
transformation for the element stiffness matrix is defined by Eq. 3.61.

$$
\mathbf{T}_{5 n \times 6 n}^{e}=\left[\begin{array}{ccc}
\mathbf{L}_{1}^{e} & & \\
& \ddots & \\
& & \mathbf{L}_{n}^{e}
\end{array}\right]
$$

$$
\mathbf{a}^{\prime} e=\mathbf{T}^{e} \mathbf{a}^{e}
$$

$$
\mathbf{f}^{\prime} e=\mathbf{T}^{e} \mathbf{f}^{e}
$$

$$
\mathbf{K}^{e}=\mathbf{T}^{e \mathrm{~T}} \mathbf{K}^{\prime} \mathbf{T}^{e}
$$

Coplanar node treatment. Alternatives to avoid the singularity of the stiffness matrix

A coplanar node is a node that has all the elements to which it belongs on the same plane. For these nodes, the $\theta_{x^{\prime}}$ and $\theta_{y^{\prime}}$ local rotations are in the same common plane. If the local system $x^{\prime}, y^{\prime}, z^{\prime}$ is chosen as the global, the projections of these rotations over the $z^{\prime}$ local axis are nulls, thus 
Chapter 3. Simucal. A footwear simulator for virtual assessment of footwear

being null the diagonal term of the stiffness matrix. This makes difficult the systematic solution of the equations system. If all the nodes were coplanar, the shell would degenerate to a plate and in this case, the stiffness matrix would be singular [85]. Therefore, if the stiffness matrix of an element with coplanar nodes is assembled in another coordinate system, a system of equations would be obtained which, although it could seem correct, would also provide a null diagonal term during the process of solving the equations since the three equations corresponding to the coplanar node would not be independent.

Of the several alternatives that exist to avoid the possible singularity of the stiffness matrix, for example, assembling in local axes, using special plate elements with rotation defined on the middle nodes of the element, or adding a fictitious rotational stiffness [85], the last one was chosen for this model. This alternative was proposed by Zienkiewicz and Taylor in [85] and it avoids the necessity to look for coplanar nodes, which considerably reduce the computational cost. The alternative introduces $M_{z}$ as a fictitious rotational stiffness and $\theta_{z}$ as an additional degree of freedom, Eq. 3.62. In this equation, the coefficient $\alpha_{n}$ and the exponent $n$ are experimental parameters, $E$ is the Young's modulus, $t$ is the thickness of the element, and $A$ is its area. Zienkiewicz and Taylor also pointed out that the values of the stiffness in this way introduced can be proportional to the values generate by the bending rotations, i.e., proportional to $t^{3}$, and that the numerical experiments proved that this scale is more insensitive to the choice of $\alpha_{n}$.

$$
\left(\begin{array}{c}
M_{z_{i}} \\
M_{z_{j}} \\
M_{z_{m}}
\end{array}\right)=\alpha_{n} E t^{n} A\left(\begin{array}{ccc}
1 & -0.5 & -0.5 \\
& 1 & -0.5 \\
\operatorname{sim} . & & 1
\end{array}\right)\left(\begin{array}{c}
\theta_{\mathbf{z}_{\mathbf{i}}} \\
\theta_{\mathbf{z}_{\mathbf{j}}} \\
\theta_{\mathbf{z}_{\mathbf{m}}}
\end{array}\right)
$$


Hence, the modified stiffness matrix used for our element is defined as next equation shows.

$$
\mathbf{K}^{e}=\left(\begin{array}{ccccccccc}
\mathbf{K}_{11}^{p} & 0 & 0 & \mathbf{K}_{12}^{p} & 0 & 0 & \mathbf{K}_{13}^{p} & 0 & 0 \\
0 & \mathbf{K}_{11}^{b} & 0 & 0 & \mathbf{K}_{12}^{b} & 0 & 0 & \mathbf{K}_{13}^{b} & 0 \\
0 & 0 & \alpha_{3} E t^{3} A & 0 & 0 & -0.5 \alpha_{3} E t^{3} A & 0 & 0 & -0.5 \alpha_{3} E t^{3} A \\
\mathbf{K}_{21}^{p} & 0 & 0 & \mathbf{K}_{22}^{p} & 0 & 0 & \mathbf{K}_{23}^{p} & 0 & 0 \\
0 & \mathbf{K}_{21}^{b} & 0 & 0 & \mathbf{K}_{22}^{b} & 0 & 0 & \mathbf{K}_{23}^{b} & 0 \\
0 & 0 & -0.5 \alpha_{3} E t^{3} A & 0 & 0 & \alpha_{3} E t^{3} A & 0 & 0 & -0.5 \alpha_{3} E t^{3} A \\
\mathbf{K}_{31}^{p} & 0 & 0 & \mathbf{K}_{32}^{p} & 0 & 0 & \mathbf{K}_{33}^{p} & 0 & 0 \\
0 & \mathbf{K}_{31}^{b} & 0 & 0 & \mathbf{K}_{32}^{b} & 0 & 0 & \mathbf{K}_{33}^{b} & 0 \\
0 & 0 & -0.5 \alpha_{3} E t^{3} A & 0 & 0 & -0.5 \alpha_{3} E t^{3} A & 0 & 0 & \alpha_{3} E t^{3} A
\end{array}\right)
$$

\subsubsection{Description of the simulation}

For this first version of Simucal, only the initial instant in which the shoe is fitted to the foot is simulated. Modeling how people introduce their feet into their shoes to put them on is very complicated. If we were able to know which the movements of the foot are when it is introduced into the shoe, it would be possible to model the behavior of the upper during this process as a forming problem in a commercial FE software. As this is an unsolved difficulty, the following procedure was chosen to model the initial foot-shoe fit.

In the initial frame (see Fig. 3.6), the meshes of the foot and shoe are aligned by the Aligner and appear superimposed in the screen. As Fig. 3.6 (left) shows, there are parts of the upper mesh that remain inside the foot mesh. These parts correspond to zones where the upper is deformed by the foot. An algorithm, explained later, that detects which nodes are inside and places them at their corresponding positions on surface was developed. 
This algorithm assumes that the foot dorsum is rigid, as the foot surface has little soft tissue, its behavior can be assumed to be determined by the behavior of the bones that form it [10]. These nodes are fundamental in the physical model because they contain all the information about the foot-shoe interaction. Their displacements are known since they are given by the difference between the position on the foot surface and the previous position inside the foot mesh.
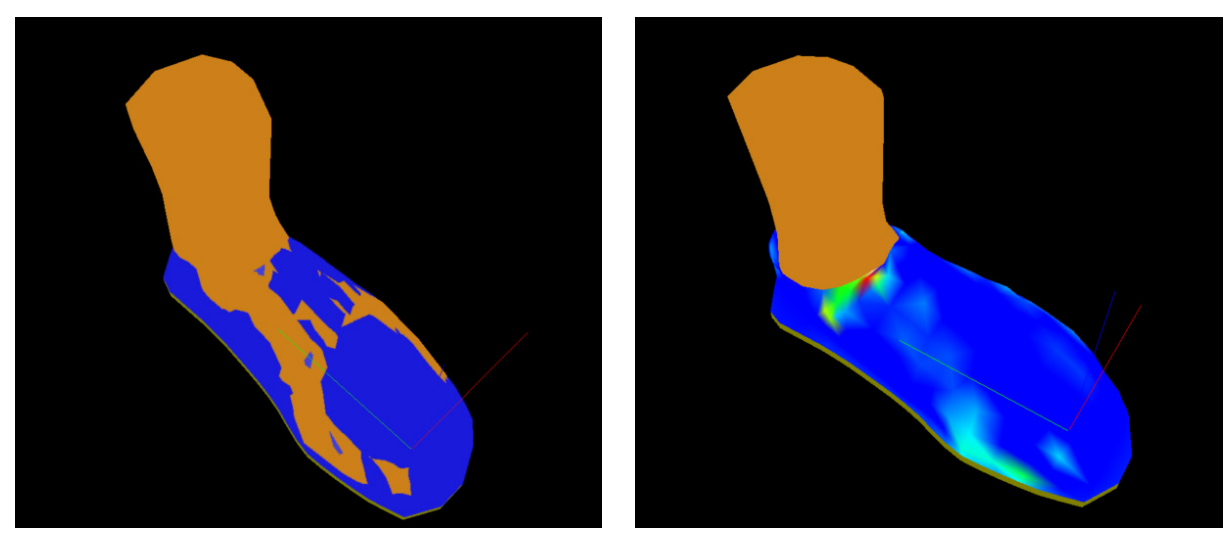

Figure 3.6: Nodes of the upper mesh inside the foot mesh in frame 0 (left). Distribution of forces normal to the upper surface in frame 0 (right).

In this initial frame, the upper deformation due to the initial fit between the foot and the shoe is compared with the upper in its non-deformed configuration. The weight of the upper is considered to be negligible because the contact between the upper and the foot are the main reasons for the deformation of the upper, thus only the external forces due to the foot-shoe interaction are taken into account. In this first version of the footwear virtual simulator, a thin sole of rubber type material was modeled and the elastic properties of this material were measured using tensile 
tests similar to those used for determining the shoe upper material properties (Section 3.3.2). Table 3.1 shows the sole properties. Although the rubber type materials usually present a hyperplastic behavior [87], in this first version of the simulator, an elastic behavior was assumed for the shoe sole as a first approximation to study its inclusion in the model.

Table 3.1: Data of the material used as shoe sole.

\begin{tabular}{ccc}
\hline Thickness (mm) & Young's modulus(MPa) & Poisson's ratio \\
\hline 4.000 & 2.231 & 0.446 \\
\hline
\end{tabular}

The plantar foot behavior cannot be assumed as the behavior of a rigid body since it is mainly formed by soft tissue. Therefore, the plantar foot should be considered as a deformable object and its deformation when it is interacting with the shoe sole should also be modeled. The fact is that this deformation is already taken into account in the model developed in this thesis because the foot will be scanned from mid-stance (when the foot leans completely on the ground), and its movement during a complete step will be rebuilt from this static position and the record of the movement of the main foot anatomical points up to push-off (see Section 4.2 in Chapter 4). During this period, the soft parts of the plantar foot that lean on the ground are already deformed by this surface in a similar way as they would be deformed leaning on a shoe sole. Therefore, this deformation is already implicit in the foot model. Thus, the nodes of the sole that remain into the foot mesh at these parts are taken out to the plantar foot following the same procedure as the nodes of the shoe upper that are inside of the foot mesh. The displacement of all these nodes are known, thus providing the boundary conditions of the problem.

Therefore, the known displacements of the upper nodes and sole nodes that 
Chapter 3. Simucal. A footwear simulator for virtual assessment of footwear

were inside the foot mesh are the boundary conditions of the problem. With these boundary conditions and taking into account that there is no interaction with the foot or with the the rest of the sole, Eq. 3.12 is applied and the rest of the node displacements are calculated. When all the displacements are determined, the loads at each node are calculated by applying Eq. 3.12 again. Thus, the diagram of the normal forces that acts on the upper surface in the position under study can be represented in the screen (Fig. 3.6 (right)).

\section{Foot-shoe initial alignment}

Fig. 3.1 has showed the basic structure of this application which was composed of two separated tools: the Aligner, whose main task is to align the virtual shoe and the scanned foot, and the Simulator, which takes the result of the Aligner as input and performs an initial fit of the virtual shoe to the scanned foot. The main task of the Simulator, the simulation process, has already been presented. In this subsection, the main task of the Aligner, the initial foot-shoe aligment, is described.

In the initial frame, the Aligner takes the design of the shoe and the mesh of the scanned foot as inputs. The Aligner assumes that the designed virtual shoe and the scanned foot both rest on the $X Y$ plane $(Z=0)$. The Aligner searches for the point of the heel of the foot, and similarly with the virtual shoe that have the minimum $x$ coordinate (see Fig. 3.7). It also looks for the middle axes of both objects, which have an angle $\theta<\epsilon$ with respect to the $X$ axis, and it projects them onto the plane $Z=0$. The middle axis of the foot is the line that goes from its minimum $x$ point to its middle point obtained using the position of the first metatarsal and the fifth metatarsal (see Fig. 3.7). However, the middle axis of the shoe is 


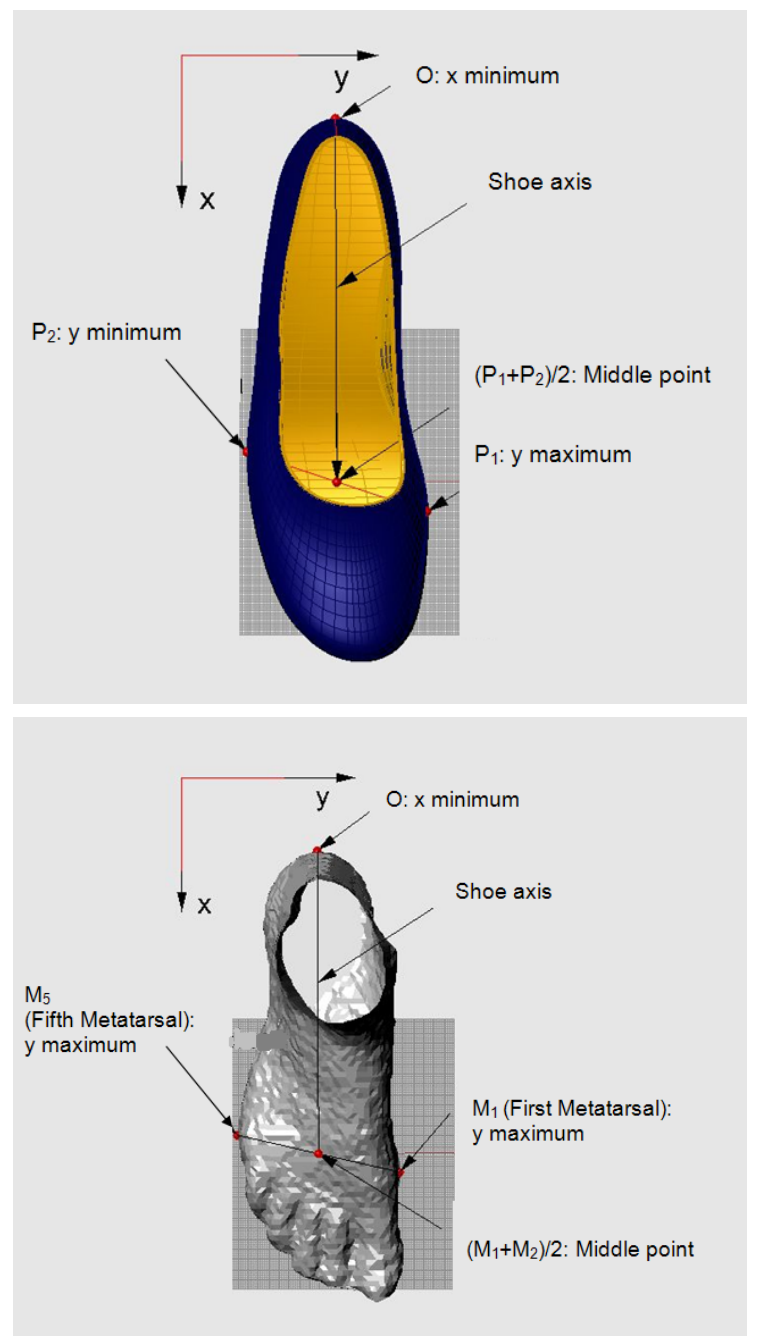

Figure 3.7: Detection of minimum $x$ coordinate point and middle axis for the shoe (top) and for the foot (bottom). Image from IBV (Institute of Biomechanics of Valencia). 
obtained with its minimum $x$ point and the middle point obtained using its minimum $y$ point and its maximum $y$ point (see Fig. 3.7). With these data, the Aligner applies to the foot a global displacement on the plane $X Y$ until the minimum $x$ point of the foot fits the minimum $x$ point of the virtual shoe. Finally, the Aligner rotates the foot, with $\mathrm{Z}$ as a rotation axis and the minimum $x$ point of the virtual shoe as the fixed point. This rotation makes the middle axes of the shoe and foot parallel. Fig. 3.8 shows an example of the initial foot-shoe alignment carried out by the Aligner.

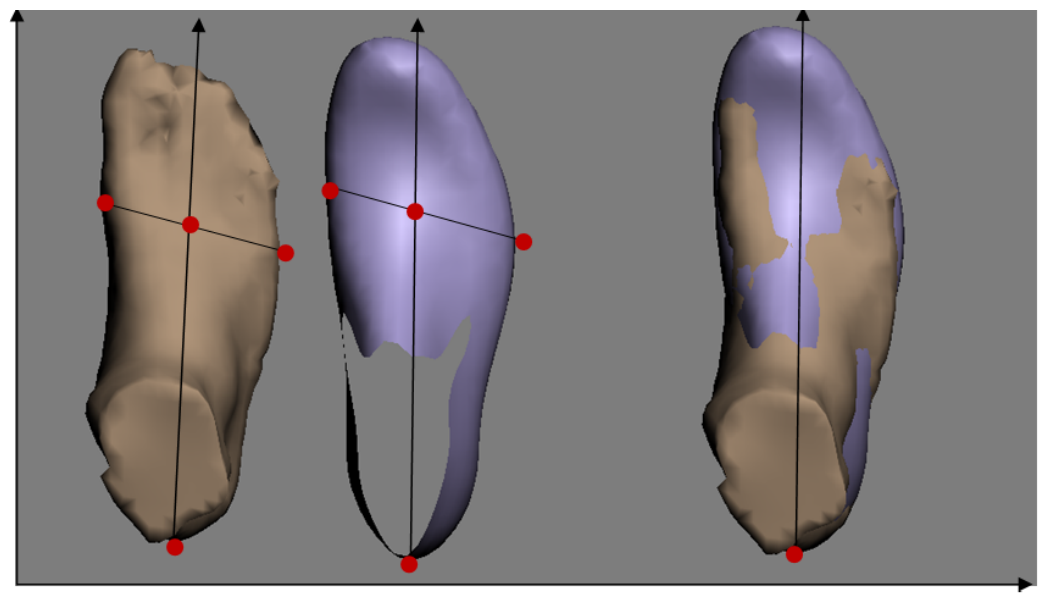

Figure 3.8: Initial foot-shoe alignment.

\section{Algorithm to extract the nodes}

The foot mesh was constructed as an enclosed surface. To detect if a node of the upper mesh is inside the foot mesh, six rays are thrown on the directions of the coordinate axis from it. Two rays are thrown on each axis, one on the positive way and another one on the negative way. If all 
the rays intersect with the foot mesh, the node is inside. To know its new position outside of the foot, it is looked for the node of the foot mesh that is closest to it and for the triangles (of the foot mesh) to which this node belongs. Several points are obtained projecting the interior node on the planes defined by these triangles. Of these points, the point that is closest and that is not out of its associate triangle is selected as the new position for the node.

With the aim of the foot mesh is not above the upper mesh, the nodes are really placed a little displaced of their new position. They are displaced on the direction of the normal vector to the upper surface. This is only for the visualization, the input to the model is always the position calculated with the procedure described above. Fig. 3.9 shows how the points from a square shell that are inside a sphere are taken outside (on the sphere surface) with this algorithm.

The described algorithm is applied iteratively since, after solving the equation system, new nodes of the upper mesh that had not been inside the foot mesh before can fall inside. In addition, some of the nodes that were taken out before solving the system can provide negative normal forces, i.e., forces that do not point toward the way in which the nodes were taken out, but towards inside the foot mesh. This means that these nodes were not taken out to a right position. Therefore, in a second iteration, the nodes that fell inside the foot mesh in the first iteration and which did not provide negative forces are kept at their current position, the new nodes that have fallen inside the foot mesh after solving the equation system are taken out, and the nodes with negative forces are released. This procedure is followed in the subsequent iterations until the convergence is reached. 
Chapter 3. Simucal. A footwear simulator for virtual assessment of footwear

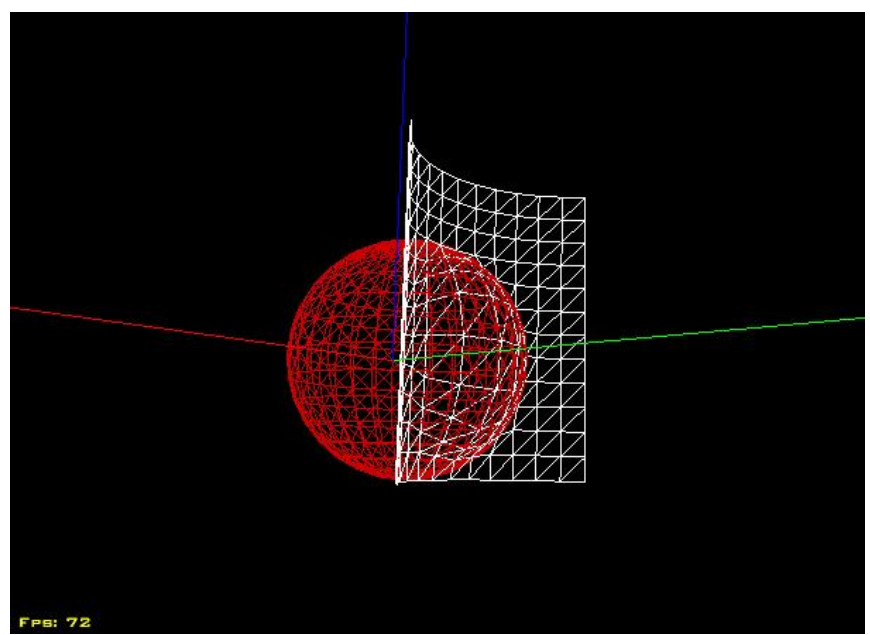

Figure 3.9: Points from a square shell that are inside a sphere, which are placed on the sphere surface with an algorithm that considers the sphere as an enclosed surface and looks for the closest point on the sphere surface to the node of the square shell.

\section{$3.3 \quad$ Results}

\subsubsection{Validation of the numerical model}

The shell model was validated with typical examples from the bibliography [88]: A clamped plate with a point load at the center (Fig. 3.10, top), the pinched hemisphere (Fig. 3.10, bottom), the pinched cylinder (Fig. 3.11, top), and the Scordelis-Lo roof (Fig. 3.11, bottom). The data of the tests are shown in Table 3.2 and the results are shown in Fig. 3.12.

In the clamped plate case, the displacement that suffers its central point when it is subjected to a vertical load of $100 \mathrm{~N}$ of magnitude was studied (Fig. 3.10, top). The theoretical displacement of this point is $0.0815 \mathrm{~m}$. In the pinched cylinder case, it was studied the deformation that suffers the 

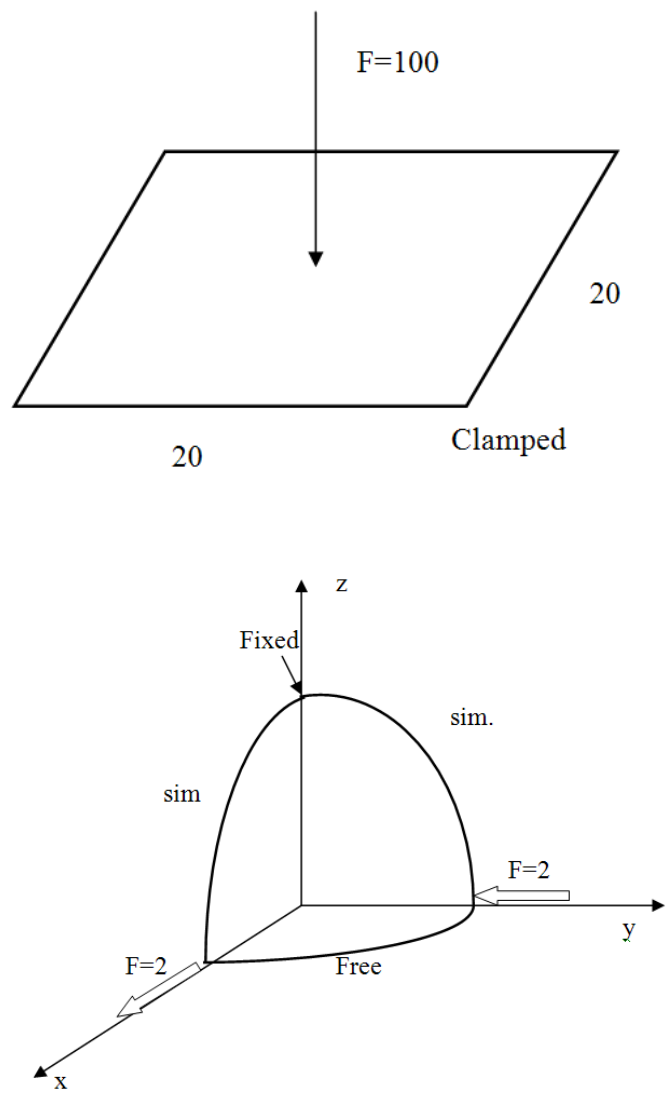

Figure 3.10: Clamped plate with a point load at the center (top) and pinched hemisphere (bottom). 

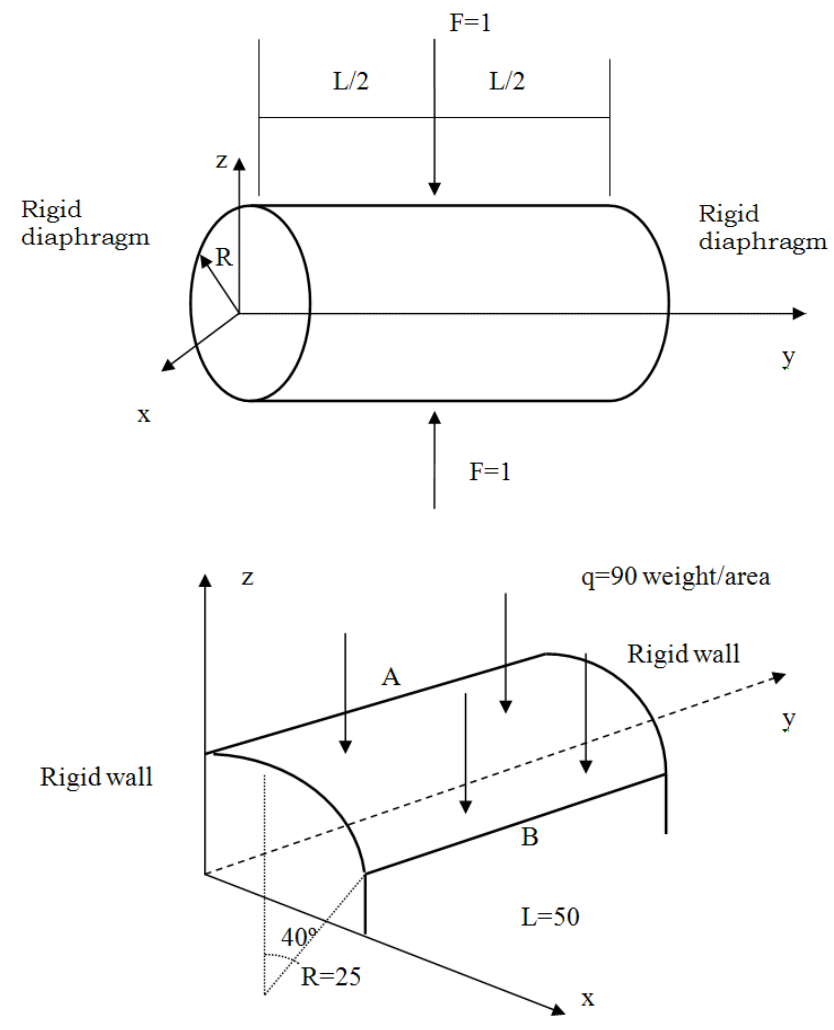

Figure 3.11: Pinched cylinder (top) and the Scordelis-Lo roof (bottom). 


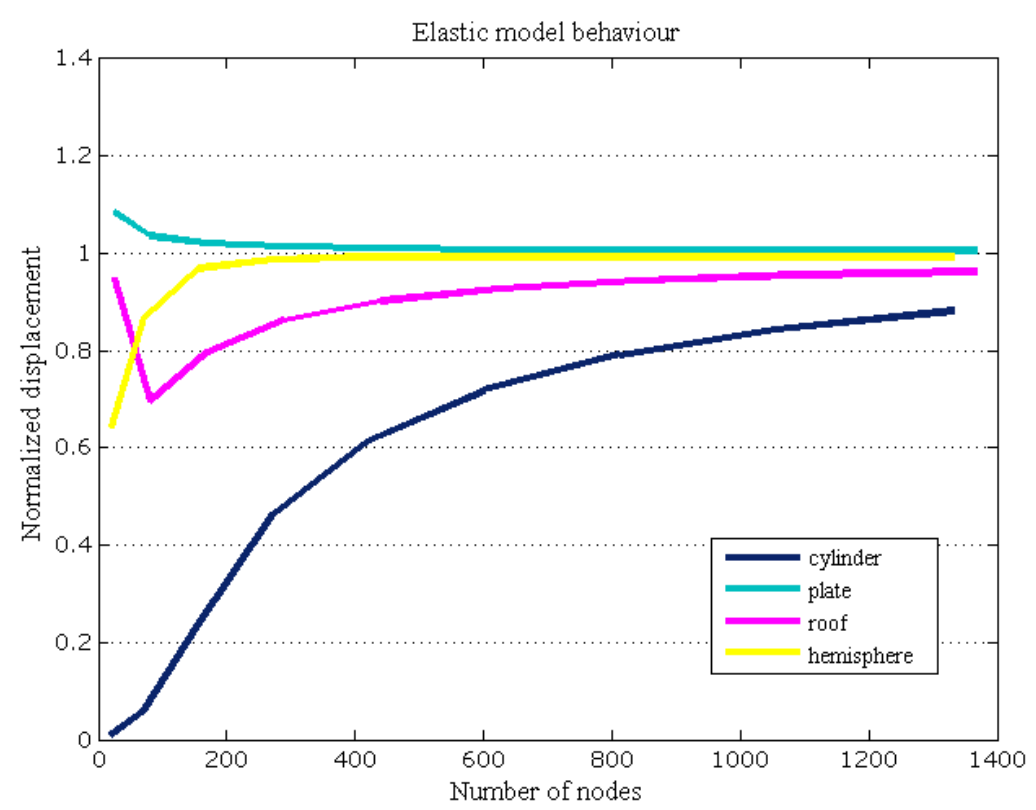

Figure 3.12: Validation of the elastic model with a clamped plate, with the pinched cylinder, with the pinched hemisphere, and with the Scordelis-Lo roof. 
Chapter 3. Simucal. A footwear simulator for virtual assessment of footwear

Table 3.2: Data of the tested meshes (S.I.).

\begin{tabular}{lc}
\hline Clamped plate & Pinched hemisphere \\
\hline Dimension $20 \times 20$ & Radius: 10 \\
Thickness: 0.1 & Thickness: 0.04 \\
Young Modulus: $30 \mathrm{e} 6$ & Young Modulus: $6.825 \mathrm{e}$ \\
Poisson ratio: 0.3 & Poisson ratio: 0.3 \\
Theoretical displacement: 0.0815 & Theoretical displacement: 0.091898 \\
\hline Pinched cylinder & Scordelis-Lo roof \\
\hline Length: 600 & Length: 50 \\
Radius: 300 & Radius: 25 \\
Thickness: 3 & Thickness: 0.25 \\
Young Modulus: 300 & Young Modulus: $4.32 \mathrm{e} 8$ \\
Poisson ratio: 0.3 & Poisson ratio: 0.0 \\
Theoretical displacement: 0.182488 & Theoretical displacement: 0.3024 \\
\hline
\end{tabular}

cylinder subjected to a couple of loads of $1 \mathrm{~N}$ of magnitude in two opposite points on one of its diameters (Fig. 3.11, top). In this case, the boundary conditions are that the cylinder is held with a rigid diaphragm. With these conditions, the theoretical displacement of these points is $0.182488 \mathrm{~m}$. The pinched hemisphere has the extreme fixed and it is subjected to a couple of forces as the cylinder but of $2 \mathrm{~N}$ of magnitude (Fig. 3.10, bottom). The theoretical solution used for normalizing the displacement is $0.091898 \mathrm{~m}$. Regarding the Scordelis-Lo roof, it represents a roof of a train coach that is subjected to its self-weight (Fig. 3.11, bottom). The boundary conditions are that the roof is joined to two rigid walls. The self-weight is 90 weight/area and the theoretical solution for the normalization of the displacements of two points on the half coach is 0.3024 . The validation of the model was done studying how the theoretical results were reached as the density of the mesh raises, i.e. as the number of nodes raises. The result obtained was divided by the real solution for normalizing the displacement, so the exact reference solution was 1 (Fig. 3.12).

The model perfectly represented the elastic behavior of the clamped plate 
and the pinched hemisphere, reaching the exact solution even with coarse meshes. The Scordelis-Lo roof solution converged early and very close to the exact solution. In the pinched cylinder case, the solution converged very slowly, approaching little by little to its exact solution as shown in Fig. 3.12.

A similar behavior was obtained for the error calculated in the strain energy. The strain energy, which is a scalar magnitude whose value is influenced by the displacements of all the nodes of the problem, was used as an error measure. The strain energy is given by Eq. 3.63, where $\sigma$ and $\varepsilon$ are the stress and strain tensors respectively, and $V$ is the volume of the shell. As the real value of this energy is not provided in the literature, it was estimated simulating the deformation of these shells in ANSYS®, a commercial FE package. With ANSYS v10, the strain energy was obtained using very refined meshes, so as to obtain a converged value for the strain energy. The error was then obtained following the definition of Eq. 3.64.

$$
\begin{gathered}
W=\frac{1}{2} \int_{v} \sigma \varepsilon \mathrm{d} V \\
\text { error }=\frac{\left|W_{\text {reference }}-W_{\text {real }}\right|}{W_{\text {real }}} \cdot 100
\end{gathered}
$$

In the case of the clamped plate and the pinched hemisphere, errors under $0.1 \%$ were obtained for meshes with a number of nodes close to 800 . In the case of the Scordelis-Lo roof, the convergence was reached for meshes with a number of nodes close to 1200 nodes. However, in the pinched cylinder 
Chapter 3. Simucal. A footwear simulator for virtual assessment of footwear

case, errors under $0.1 \%$ were only obtained for meshes with a number of nodes above 3000 .

To apply the physical model to these meshes, a value for $\alpha_{3}$ was needed (Section 3.2.2). For these meshes, the only case in which there are coplanar nodes is the clamped plate case. In fact, all the nodes are coplanar in this case, so an analysis of different values for $\alpha_{3}$ was carried out for this example. It was found that with the alternative proposed by Zienkiewicz and Taylor in [85], the exact result was always obtained, at least, for $\alpha_{3}$ values between 0 and 1 .

For the virtual shoes used in our experiments, a search for coplanar nodes was carried out for the upper mesh. It was found that there were no coplanar nodes in the tested meshes. These meshes were not made without coplanar nodes intentionally, but they were made following a basic design of shoe. So, taking into account the results obtained for the clamped plate mesh, the value 0.001 was chosen for $\alpha_{3}$.

\subsubsection{Behavior of the materials}

To validate the approximation to a linear elastic behavior for simulating the real behavior of the shoe upper, three materials were analyzed. Three kinds of leathers commonly used in the manufacture of shoe uppers were provided by a footwear manufacturer. Four samples of each material were subjected to tensile tests according to the UNE EN ISO 527-1 standard using a universal test machine MIB-60-AM. These tests were carried out at the Physical-Mechanical Characterization Laboratory of the Institute of Material Technology of the Universidad Politécnica de Valencia.

In order to obtain their elastic parameters, the stress versus the longitudinal strain and versus the lateral strain were measured (Fig. 3.13). Accord- 

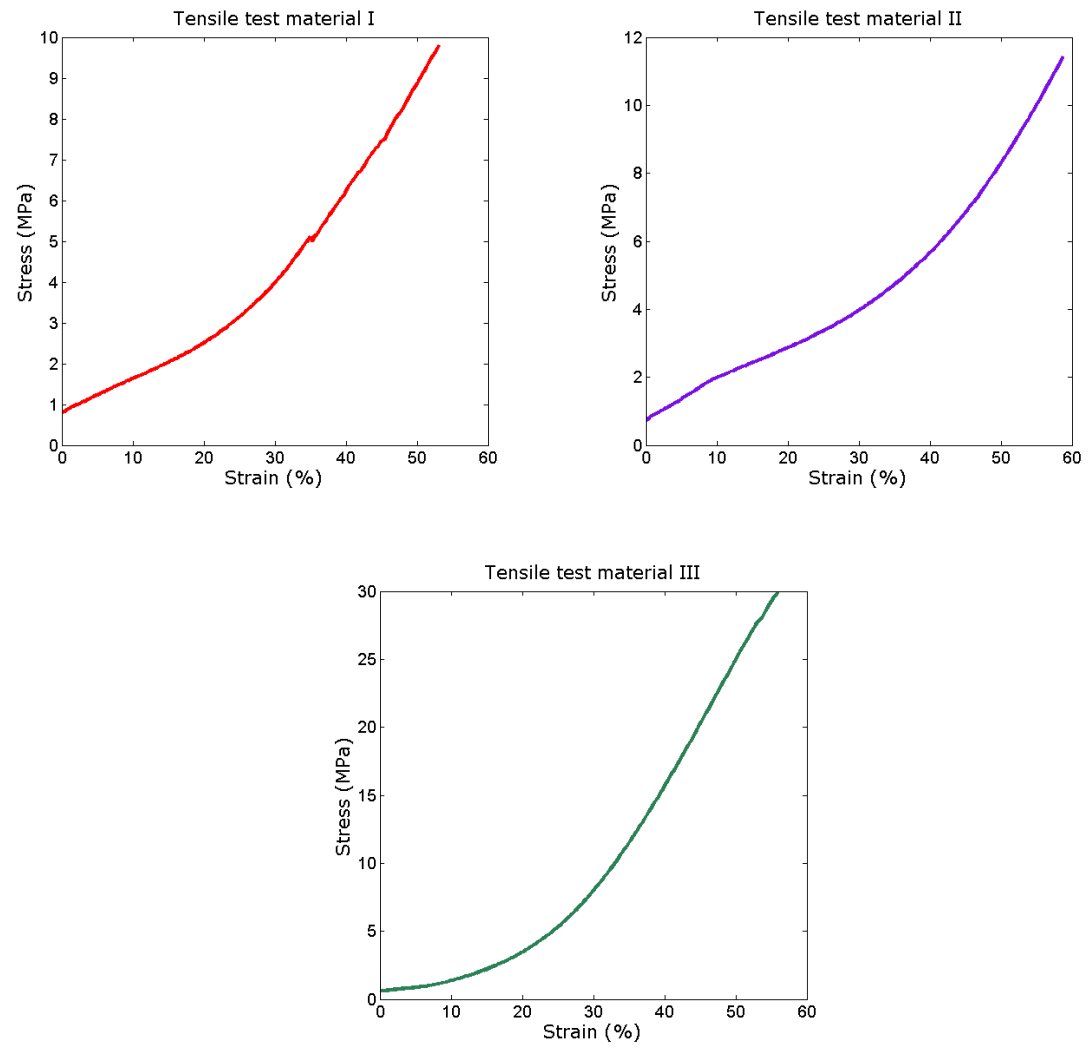

Figure 3.13: Tensile test results for three leathers used as shoe uppers in this work. 
Chapter 3. Simucal. A footwear simulator for virtual assessment of footwear

ing to Mitton [89, 90], on initial monotonic loading for a typical leather test, the stress increases proportionally with elongation up to a level of $20 \%$. Therefore, a linear fitting of the stress-strain curves was made at their beginning, thereby obtaining the Young's modulus and the Poisson's ratio of each material for these zones (Table 3.3).

Table 3.3: Data of the leathers used as shoe uppers.

\begin{tabular}{|c|c|c|c|}
\hline Material & Thickness (mm) & Young's modulus(MPa) & Poisson's ratio \\
\hline I & 0.65 & 8.489 & 0.061 \\
\hline II & 0.76 & 12.904 & 0.015 \\
\hline III & 0.77 & 5.783 & 0.056 \\
\hline
\end{tabular}

Few references about the study of the interaction between the foot and the shoe upper have been found in the literature. However, based on the data provided by the few authors who have studied these pressures, Wilson et al. [91], who obtained peak stresses of about $150 \mathrm{kPa}$ during the step, Olaso et al. [92] who obtained peak stresses of about 200kPa, Jordan et al. [2] who obtained peak stresses of about $46 \mathrm{kPa}$, and Rupérez et al. [44] who obtained peak stresses of about 400kPa (Fig. 4.7, Section 4.3, Chapter 4 ), in the present work it was assumed that the foot would exert stresses close to these values. Therefore, the study of the behavior of the upper at the linear range of these curves would be justified, especially, taking into account that a linear fitting under $20 \%$ of the elongation provides stress values around $2 \mathrm{MPa}$ in all the cases, which is much more greater than $400 \mathrm{kPa}$. It should also be pointed out that the leathers used as shoe uppers in these experiments were similar to those provided by the footwear manufacturer for the experiments described in Chapter 5, which provided peak pressures of about $400 \mathrm{kPa}$ (Section 4.3). The elastic behavior was considered to be $10 \%$ of the longitudinal strain which still provided stresses much greater than $400 \mathrm{kPa}$. 
3.3. Results

\subsubsection{Simulation}

Three test meshes were created using 3DStudioMax one for the foot, one for the sole, and another one for the upper (Fig. 3.14). The initial fit between the foot and the shoe was simulated. The position was the mid-stance, i.e. when the foot leans completely on the ground.

In this first version of the simulator, the upper mesh was not made very dense to reduce the computational cost that the model requires. This cost is approximately $N^{3}$, where $N$ is the unknown displacement number. The resolution of the system of equations is the main contribution to the computational cost, for which the free libraries CLAPACK were used.

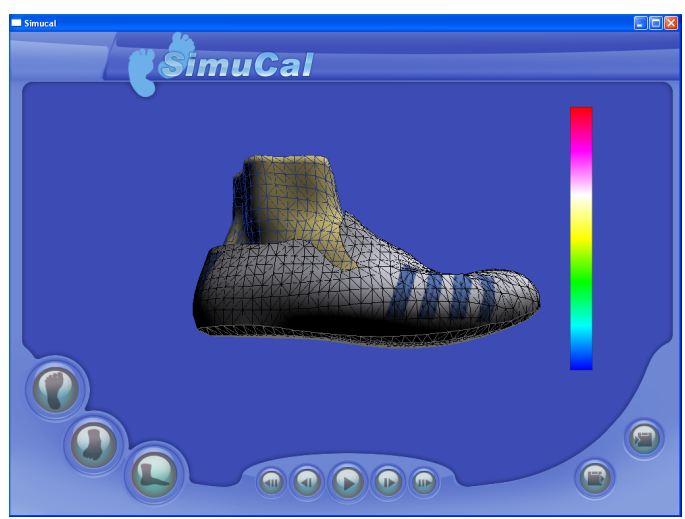

Figure 3.14: Meshes for the foot, for the shoe upper, and for the shoe sole.

After calculating the unknown displacements and placing each node at its new position of the deformed state, it was observed that there were new nodes inside the foot mesh as well as some nodes provided negative normal forces. To solve the problem that this generates, the algorithm that takes the nodes out must be applied again, forcing these new nodes and the nodes that there were previously inside the foot mesh and did not provide negative forces to go to the corresponding positions on the foot 
Chapter 3. Simucal. A footwear simulator for virtual assessment of footwear

surface, and the nodes that provide negative normal forces are released, thus displacements are solved again applying the Eq. 3.12. This process is repeated until there are no nodes inside the foot mesh and no nodes provide negative normal forces (Section 3.2.3). This process increases the computational cost to approximately $k N^{3}$, where $k$ is the number of times that the Eq. 3.12 is applied in that frame, although $N$ is reduced a little because the number of known displacement increases.

The memory cost depends mainly on the size of the matrices. The greatest of all is the stiffness matrix, its size is $n \times n$, where $n$ is the number of nodes multiplied by 6 (the number of degrees freedom of the nodes). As three matrices of that size were needed, the memory cost was $O\left(3 n^{2}\right)$.

Once the displacements are calculated, the physical model provides all the forces and momenta that act at the upper due to the interaction with the foot. However, the pressures that can really be measured with the current available sensors designed for measuring the pressures on the foot surface while walking is the pressure normal to the foot surface. Therefore, the distribution shown in the simulation is the force normal to the upper surface (Fig. 3.15, bottom). The normal pressure corresponds to the dorsal pressure, which can be measured with any kind of sensor since it will be obtained dividing this force by the sensor surface (Eq. 3.65).

$$
\text { Pressure }=\frac{F_{\text {normal }}}{S_{\text {sensor }}}
$$

Fig. 3.15 shows two images at the top corresponding to the two screens of the user interface developed for Simucal, and two views at the bottom corresponding to the simulation of the initial fit. In this first version of Simucal, the user interface allows to select the material of the upper and 
3.3. Results
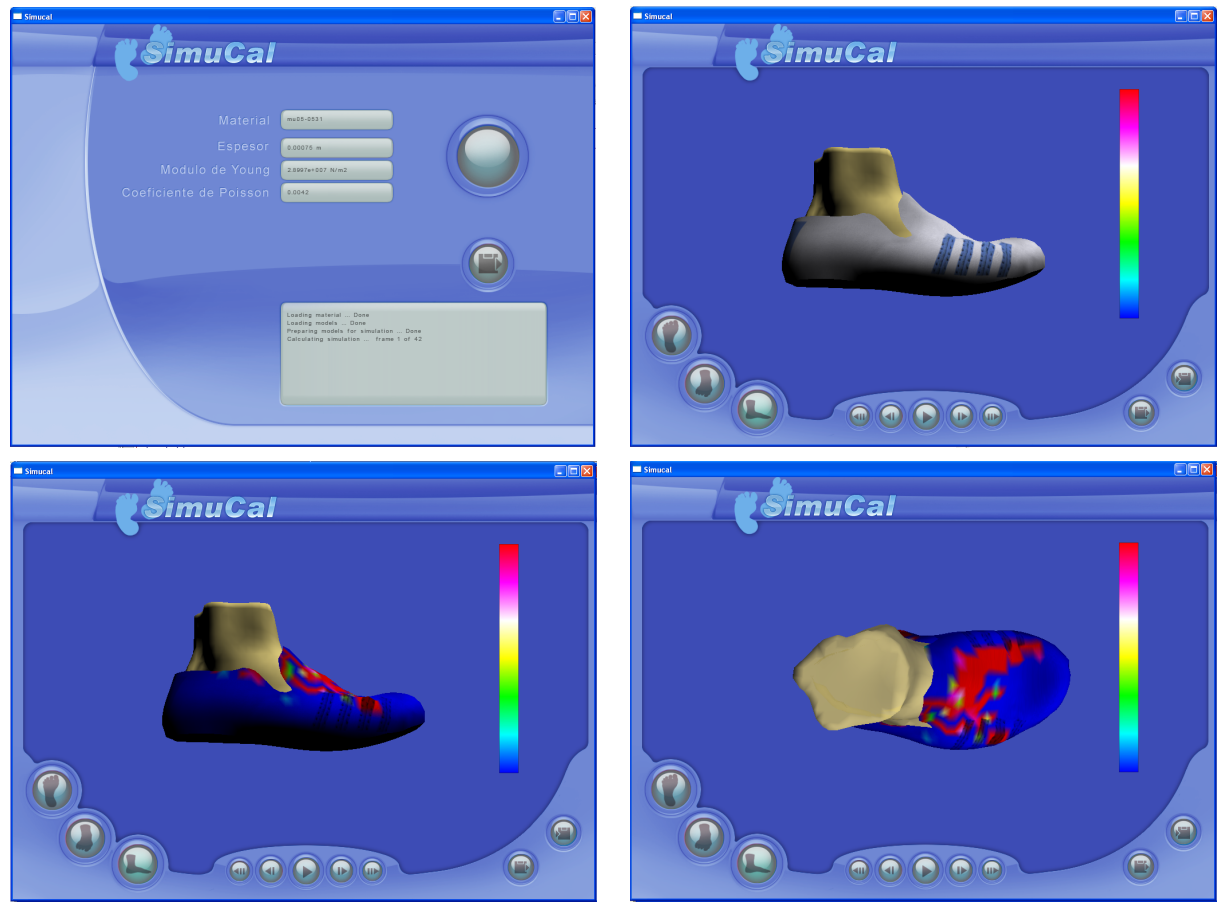

Figure 3.15: Simulation of the initial fit between the shoe and the foot for material I where the normal force distribution is shown in colors in the images at the bottom of the figure. The initial screen of the user interface is shown in the top left image, and the second screen of the user interface is shown in the top right image. 
Chapter 3. Simucal. A footwear simulator for virtual assessment of footwear

to visualize the foot from any perspective. It also allows to save and to load the simulation and to place the shoe in any position. At the bottom of this figure, it can be observed the distribution of contact forces between the foot and the upper generated in the initial frame for material I, where the color bar is an indicator of the intensity of the normal force that acts at each node.

\subsection{Discussion}

Despite the developments shown here, some issues would need to be addressed which are commented in this section. The choice of the alternative proposed by Zienkiewicz and Taylor, which introduces $\theta_{z}$ as an additional degree of freedom for all the nodes (Section 3.2.2) is considered to be the best to minimize the computational cost that the model requires. A coplanar node has all the elements to which it belongs on the same plane [85]. Analyzing whether this occurs for each node of a mesh is very costly. The coplanar nodes can introduce singularities in the model when their associated stiffness matrices are changed from local coordinates to global coordinates (Section 3.2.2). There are alternatives that propose localizing these nodes to build special matrices of change of coordinates. Associating $\theta_{z}$ as a fictitious degree of freedom for all the nodes leads to the same change matrix for all the $\mathbf{K}$ matrices [85]. Therefore, looking for the coplanar nodes is not necessary and the computational cost is reduced.

The value of $\alpha_{n}$ can be arbitrary because coplanar nodes do not exist in the tested mesh. The fact that the value of $\alpha_{n}$ can be any value reduces the computational cost. Coplanar nodes are not expected to be in the mesh of the upper of a shoe since the upper has to be curved to cover the foot surface. However, if there were coplanar nodes in the mesh, then a 
study of the results with different values of $\alpha_{3}$ would have to be done in order to choose the appropriate value.

The resolution of the system of equations is what most contributes to the computational cost. The computational cost is difficult to reduce. The cost of the majority of the algorithms that solve systems by the direct method is on the same order as the LU algorithm, $N^{3}$. The CLAPACK libraries were chosen due to its easy implementation and its fast results.

In Simucal, the footwear assessment will be based on fitting a shoe to a certain quantity of feet of the same size but with different forms. Therefore, an automatic alignment between the foot and the shoe is needed. This automatic alignment has been based on fitting first the rearfoot to the shoe, later aligning the middle axes of both objects foot and shoe. As the application developed in this thesis is the first one in simulating the physical behavior of the shoe upper, no previous alignment between the foot and the shoe that takes into account the shoe upper has been found in the literature. This approximation to the real way in which individuals put their shoes on should be validated, and its validation will represent a great difficulty when specific real cases want to be assessed because it strongly depends on the individual. However, the main approach of the application developed in this thesis is to provide footwear manufacturers with a tool which allows them to assess their designs with a great quantity of feet with different sizes and form. Thus, the automatic approximation to the real foot-shoe alignment presented in this chapter is of great value for the developed application.

The simulation could also have been solved approaching the problem from the point of view of Contact Mechanics. Contact Mechanics has been developed for solving problems of contact and impact between solids with and without friction $[93,94,95,96]$. Most of the time, the Finite Elements 
Chapter 3. Simucal. A footwear simulator for virtual assessment of footwear

Method (FEM) is a part of the development along with some methods for introducing the restrictions due to the contact [97]. Of these methods, the Lagrange multiplier method would be the most interesting to solve this problem since directly would provide the contact forces. The Lagrange multipliers are interpreted as the necessary forces for maintaining the restrictions at the contact, so they directly would provide forces and pressures at these areas.

Regarding the inclusion of the sole into the model, this first approximation to the behavior of a thin shell is needed in order to achieve a realistic simulation for the footwear performance. Different shoe sole shapes must be introduced just as their real behaviors, even shoes with high heels will be modeled in a future to complete the actual purpose of this footwear simulator.

In future work, the simulation of a complete step will be simulated. A similar procedure will be carried out for each frame but taking into account large deformations since these are the kind of deformations suffered by the upper during a complete step.

\subsection{Summary}

The present chapter has described a computational application developed to virtually assess footwear, Simucal. It also describes a preliminary computational model for the shoe upper behavior that can estimate the pressure distribution on the foot surface while walking. Although some of the assumptions are still restrictive in this first version of the simulator, Simucal will allow the footwear manufacturer to analyze virtually the functionality of a footwear design. In the future, the preliminary model developed for the 
shoe upper in the simulator will serve as the basis for the development of subsequent models, which will be able to analyze a more realistic behavior of the footwear materials, using non-linear models, considering heterogeneous materials, and simulating large deformations during a complete step. 
Chapter 3. Simucal. A footwear simulator for virtual assessment of footwear 


\section{Chapter 4}

\section{Foot animation}

Establishing the appropriate pressure exerted by the shoe upper over the foot surface is important for the design of specific footwear, although measuring these dorsal pressures can also provide important additional information. In previous chapters, a virtual simulator to perform studies of comfort and functionality in CAD-CAE footwear design was presented. This chapter describes the procedure carried out to obtain the foot animations which will be used in this simulator. The virtual feet used in the simulator will be feet without a standard form scanned in a static way. Their movements will be rebuilt from the record of movements of several foot anatomical points during a complete step. The dorsal pressures exerted by some shoe uppers on these anatomical points were measured for several subjects and used to establish the viability of the use of these animations in a virtual simulator for footwear [44]. 


\subsection{Introduction}

As it was shown up in Chapter 2, the interaction between the foot and the shoe sole has been widely studied and modeled. However, few references about the study of the interaction between the foot and the shoe upper have been found in the literature. In 1995, Jordan and Bartlett [2] performed a study using three kinds of casual shoes to establish the relationship between short-term perceived comfort and pressure distribution on the dorsal and plantar surface of the foot while walking. They suggested that the measurement of the pressure distribution at the foot-shoe interface could be a useful tool in identifying the causes of discomfort in footwear. Later in 1997, Jordan et al. [98] repeated the study with ten shoes. Their new study showed that the perceived comfort at the plantar and dorsal surfaces could be related to peak pressure and maximum force in all foot regions, but these relations varied depending on the region. In 2007, Olaso et al. [92] performed a study to analyze the pressure distribution exerted by mountain boots on the dorsal side of the foot. They concluded that dorsal pressure caused by footwear directly depended on the fit between foot and shoe as well as the activity that was being performed. However, no models that simulate the physical behavior of the shoe uppers when they come into contact with the foot had been developed before the development of Simucal, the footwear virtual simulator presented in this thesis. In the simulator, a model to simulate the deformation of the shoe uppers in the initial fit was developed using the Finite Element Method (Chapters 3). In a future, a model will be develop which enable the simulator to show how the shoe upper is deformed while walking, and therefore, the assessment of a prototype will be not only static, but also dynamic. As a consequence, the simulator will need to have access to a database of virtual feet and to have access to their corresponding movements during a complete step, i.e., 
to have access to foot virtual animations.

This chapter describes the procedure carried out to obtain the foot animations that will be used in the simulator. These animations are a fundamental tool to perform dynamic assessments of the footwear behavior. The dorsal pressures exerted by five leathers used as shoe uppers on several foot anatomical points of four subjects were measured in order to study the viability of the use of this kind of animations in a virtual footwear simulator.

Section 4.2 describes how the foot animation is obtained. Section 4.3 describes the process carried out to measure the pressures on the foot surface (dorsal pressures). Finally, Section 4.4 explains how the foot animation obtained is a viable alternative to be introduced into the simulator as the movement that the foot performs in a complete step.

\subsection{Obtaining the foot animation}

The 3D animation of the foot was obtained from four volunteers aged 2731 using the same size of shoe. The volunteers were recruited using an advertisement, gave informed consent, and were paid after performing the task. The foot animation was obtained by integrating the static information about the 3D foot form and the dynamic record of the movement of several key anatomical points of the foot during a complete step. The data collection was carried out at the IBV, Institute of Biomechanics of Valencia (http://www.ibv.org/). 
Chapter 4. Foot animation

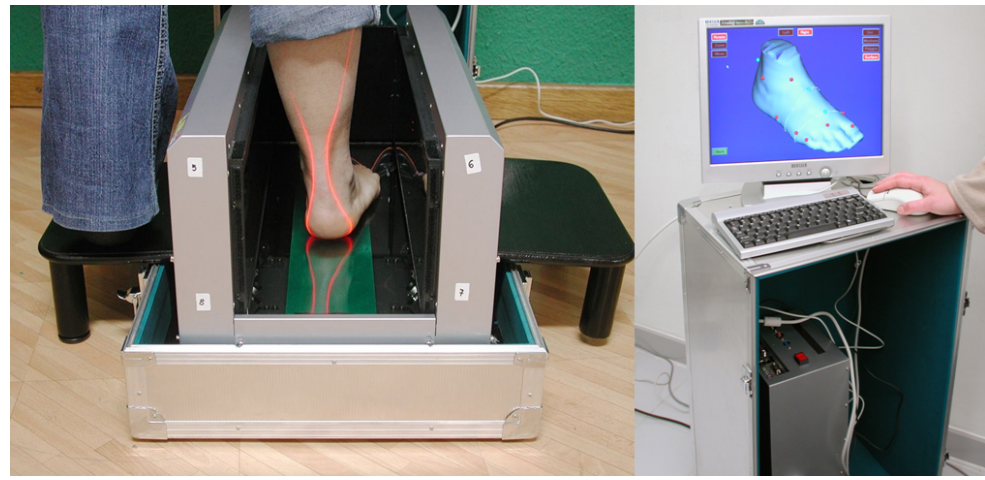

Figure 4.1: 3D static foot form record. Image from $I B V$.

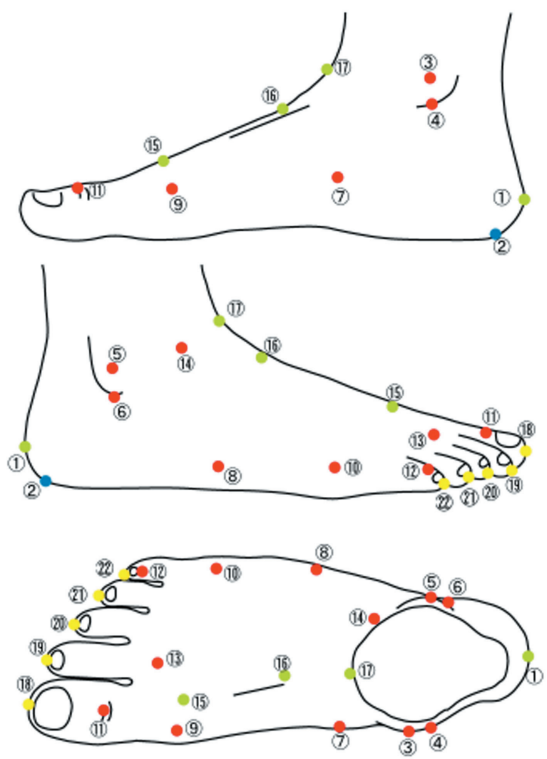

\begin{tabular}{|c|c|}
\hline 1 & Pternion \\
\hline 2 & Landing point \\
\hline 3 & $\begin{array}{l}\text { The most medial point of medial } \\
\text { malleolus }\end{array}$ \\
\hline 4 & Sphyrion \\
\hline 5 & $\begin{array}{l}\text { The most lateral point of lateral } \\
\text { malleolus }\end{array}$ \\
\hline 6 & Sphyrion fibulare \\
\hline 7 & Navicular \\
\hline 8 & Tuberosity of 5th metatarsalis \\
\hline 9 & Metatarsale tibiale \\
\hline 10 & Metatarsale fibulare \\
\hline 11 & Toe \#1 joint \\
\hline 12 & Toe \#5 joint \\
\hline 13 & Head of 2nd metatarsal bone \\
\hline 14 & Tentative junction point \\
\hline 15 & Top of Ball Girth point \\
\hline 16 & Top of Instep point \\
\hline 17 & $\begin{array}{l}\text { Junction point(Tentative point is } \\
\text { needed) }\end{array}$ \\
\hline 18 & Tip of \#1 toe \\
\hline 19 & Tip of \#2 toe \\
\hline 20 & Tip of \#3 toe \\
\hline 21 & Tip of \#4 toe \\
\hline 22 & Tip of \#5 toe \\
\hline
\end{tabular}

Figure 4.2: Key anatomic landmarks (Image from Infoot de I-ware Laboratory). 


\subsubsection{Static 3D foot form}

The 3D static foot form was obtained using the techniques provided by García-Hernández et al. in 2005 [99] to build a 3D static foot database at the IBV (Institute of Biomechanics of Valencia). It was used a scanner (Infoot of I-Ware Laboratory) that consists of two laser diodes and eight cameras. This scanner registers a point cluster that defines the foot surface when individuals are standing up and distributing their weight equally on their feet (Fig. 4.1). The scanner also makes a record of fifteen anatomical landmarks that previously are placed on the foot surface by expert anatomists. Fig. 4.2 shows a scheme of the anatomical points on which the landmarks were placed. The fifteen anatomical points are points from 1 to 14 , and point 17 . Points 15 and 16 are not placed by the anatomist, but they are calculated later from anthropometric measurements and the dimensions obtained from the cloud of points [100], as well as the tips of the toes.

\subsubsection{Foot movement record}
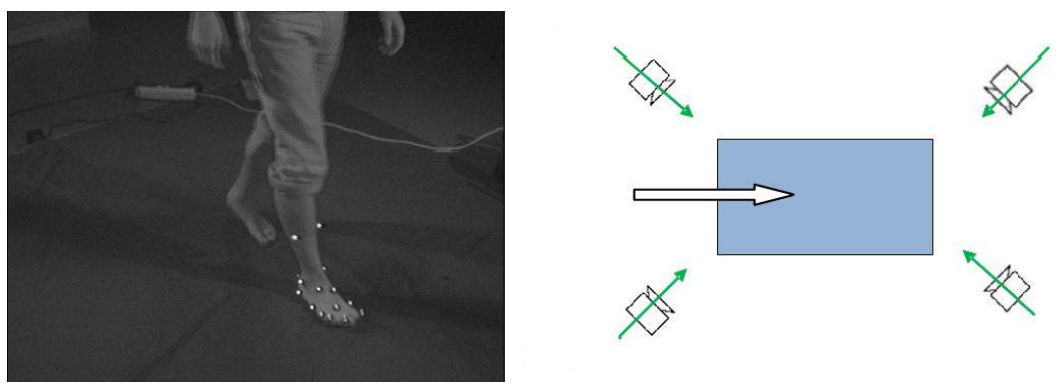

Figure 4.3: Foot movement analysis system. Image from IBV

The foot movement analysis system consisted of four cameras CCTV (b/w) 
$(\mathrm{CCD}$ of $681(\mathrm{H}) \times 582(\mathrm{~V})$ pixels, $500(\mathrm{H}) \times 400(\mathrm{~V})$ of resolution, PAL (625/50/25), 0.05cd of minimum illumination and 1/250s, 1/500s, 1/1000s of obturation speed) with a frame rate of 200 frames/second. Each camera worked together with an infrared lamp that improves the contrast of the reflecting landmarks in the dark. To build the foot movement animation during the step, the static foot model and the dynamic foot model obtained from the record of the four-camera system were integrated using a procedure similar to the procedures described by Carson et al. [101] and by MacWilliams et al. [102]. Each subject was required to walk on a platform (Fig. 4.3) and the coordinates of the fifteen landmarks during the time of the lean phases were recorded by the four-camera system. A Procrustes alignment [103] (minimization of the square-distance sum between homologous landmarks) of the static model and the dynamic model was applied using the positions of the anatomical landmarks registered in both models. After that, an exponential smoothing algorithm was implemented to deform the static foot mesh based on the movement of the fifteen anatomical points plus five more, which were added later (using the same procedure that in the static model) to define the zones that were not well defined, e.g., the toe zones (Fig. 4.2).

The foot animation during a step was obtained for each subject following the procedure described. This procedure was carried out in MATLAB and the generated animation was exported as .stl files to 3DStudioMax to apply the textures. Fig. 4.4 shows one of them. The animation simulates the gait of a subject and consists of a set of meshes, each of which represents the foot position at the corresponding stage of the step. The idea is to introduce this animation in the simulator as the generic gait for a given subject and use it to assess different kinds of shoes. In the present study, five shoes made from the same design but manufactured with five different shoe upper materials were assessed. To introduce the foot animation as 
the generic gait of a subject in the simulator, the gait cycle of that subject should be similar in all the cases i.e., wearing any of the five shoes analyzed. To assure this, the dorsal pressures obtained for each subject were analyzed.

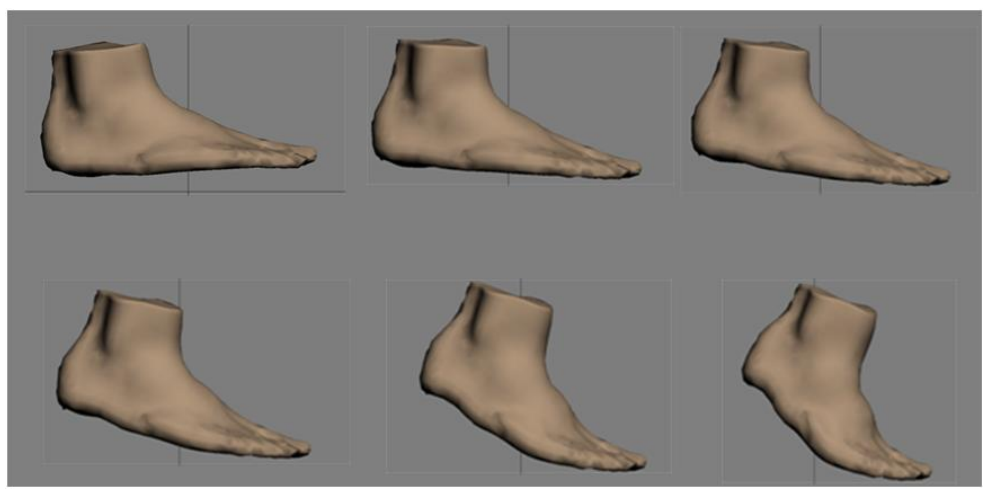

Figure 4.4: Foot animation.

\subsection{Dorsal Pressures}

The four volunteers were required to walk on the same platform used for the movement record wearing five different kinds of shoes. They followed a self-selected velocity. A footwear company manufactured five shoes from the same exact design but using a different shoe upper material for each one. Subjects wore standard socks and the fit was controlled by a strip with a hoop-and loop fastener at its end that the users fitted their selves (Fig. 4.5).

The shoes were manufactured without both reinforcements or seams since only dorsal pressures for homogeneous uppers were studied in this thesis. 


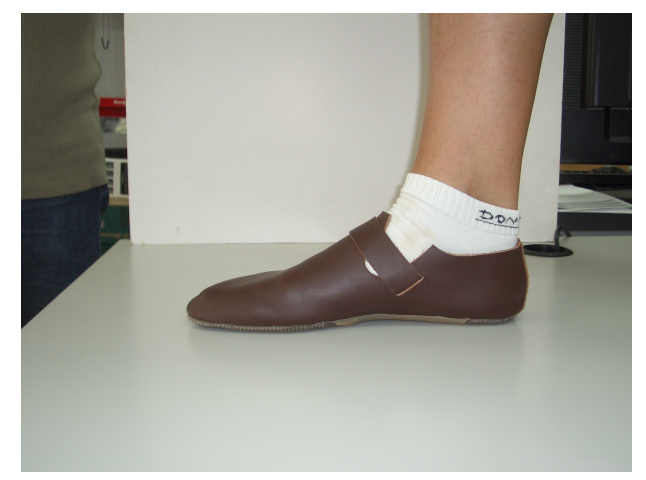

Figure 4.5: Kind of shoe used in the experiments.

Therefore, only homogeneous and isotropic materials, which were characterized by three parameters: the Young's modulus, the Poisson's ratio, and the thickness, were used to manufacture the shoes. These parameters were obtained by the IBV and they are shown in Table 4.1. The samples used to carry out these tests were cut from the same leather's piece used to manufacture the shoes. The shoes were new, there was no previous period of use, and given the short period of time during which the shoes were used in the experiment, the same shoes were used for all the participants.

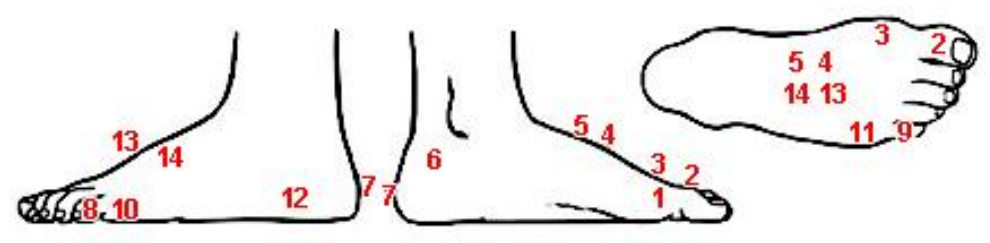

Figure 4.6: Distribution of the pressure sensors on the foot surface. Image from $I B V$.

In a separate trial, each subject was required to walk on the same platform used for the movement record wearing each one of the five shoes. The dorsal pressures were measured for each subject and each shoe by 
the IBV. Fourteen pressure sensors (TEKSCAN Flexiforceß) that gather both static and dynamic pressures were connected to a computer through wireless technology. These sensors were placed on fourteen of the twenty anatomical points used to create the animation (Fig. 4.6). The sensors were placed on the foot surface between the foot and the shoe upper on these points which are bones or joints with behavior of rigid-body that will not change in gait wearing a shoe. All the subjects performed at least two valid trials of three steps with each shoe, and the mean of the pressure collected for each sensor in each frame of each step was calculated.

Table 4.1: Mechanical properties of the leathers used as shoe upper materials. Data provided by the $I B V$.

\begin{tabular}{lccc}
\hline Material & $\begin{array}{c}\text { Thickness } \\
\text { mm }\end{array}$ & $\begin{array}{c}\text { Young's Modulus } \\
\text { MPa }\end{array}$ & Poisson's Ratio \\
\hline MU05-0531 & 0.75 & 28.997 & 0.042 \\
MU05-0532 & 0.64 & 9.297 & 0.047 \\
MU05-0533 & 0.77 & 8.616 & 0.064 \\
MU06-0279 & 1.00 & 126.085 & 0.238 \\
MU06-0531 & 2.00 & 33.929 & 0.085 \\
\hline
\end{tabular}

The dorsal pressures exerted by the shoe upper materials over the foot surface were measured for the four subjects. Firstly, the peak pressure distributions obtained by each sensor for each subject and each material were plotted (Fig. 4.7). The purpose was to study if the maximum values of these pressures were similar to the results found in the literature. They were indeed similar to those values obtained by Wilson et al. [91], who obtained peak stresses of about $150 \mathrm{kPa}$ during the step, Olaso et al. [92] who obtained peak stresses of about $200 \mathrm{kPa}$, and Jordan et al. [2] who obtained peak stresses of about $46 \mathrm{kPa}$ (Section 3.3.2, Chapter 3). In these curves, it was also observed that the distribution of the pressure peaks measured for a given subject presented a similar pattern for all the mate- 
rials, suggesting that the gait of the subject was similar wearing any of the shoes. However, these data were not conclusive since they corresponded to maximum values of the pressure, which depend on the material properties as well as on the anatomical points on which they were collected. In fact, the differences in the values of the reached pressure peaks were related to the different stiffness of each material. The stiffer the materials (higher Young modulus, Table 4.1), the higher the peaks.
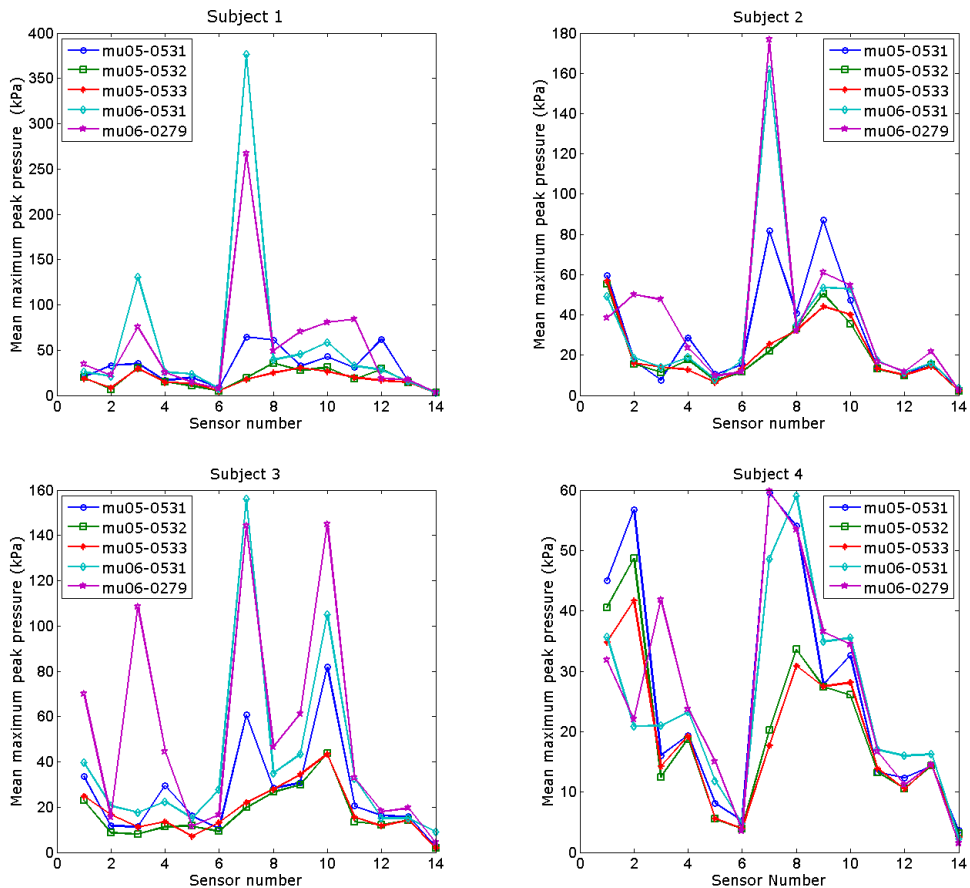

Figure 4.7: Peak pressure distributions along a complete step for all the subjects on all the sensors.

To confirm that the gait of the subject was similar wearing any of the shoes, 

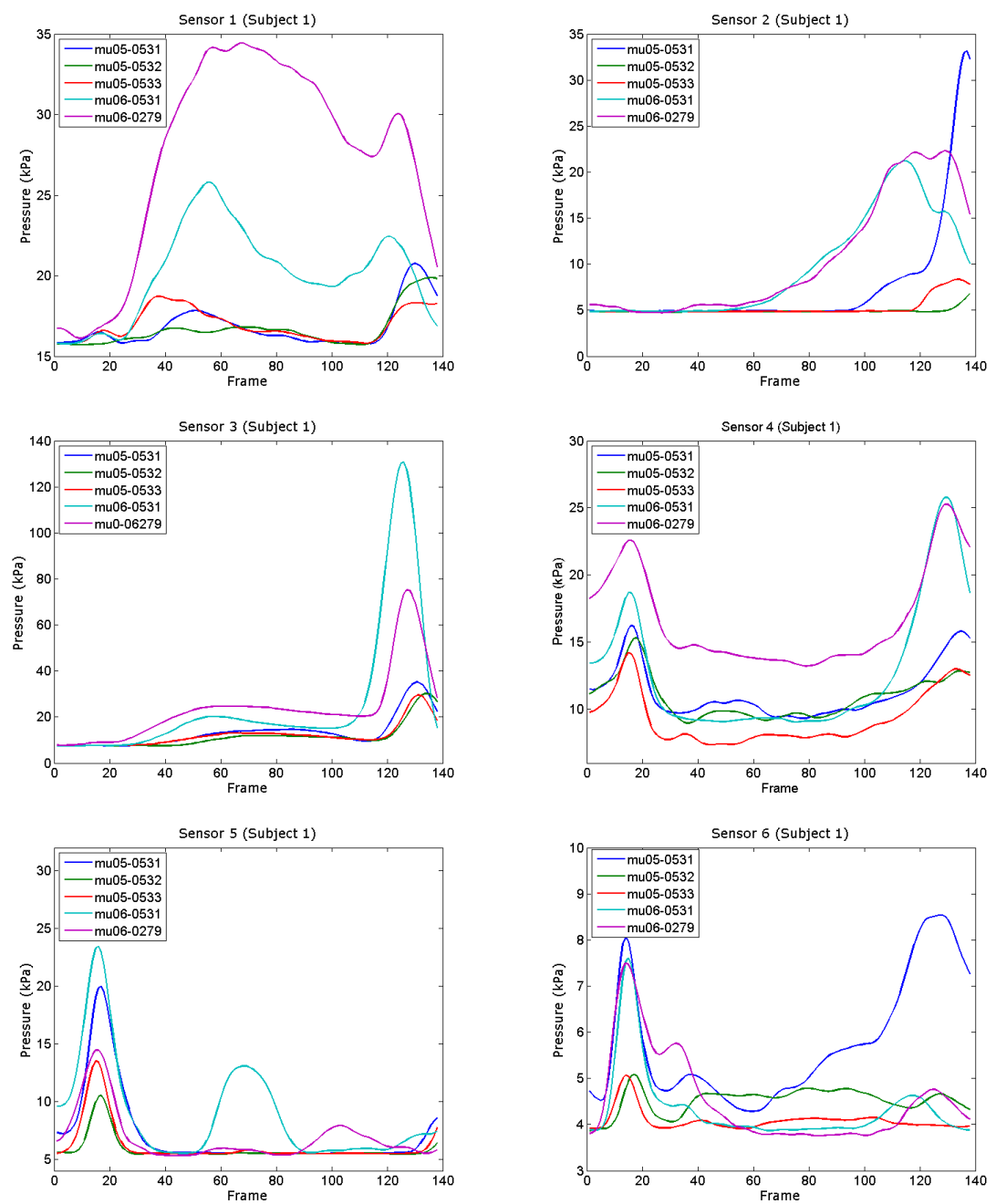

Figure 4.8: Dorsal pressure distribution for subject 1, sensor 1 to sensor 6 . 

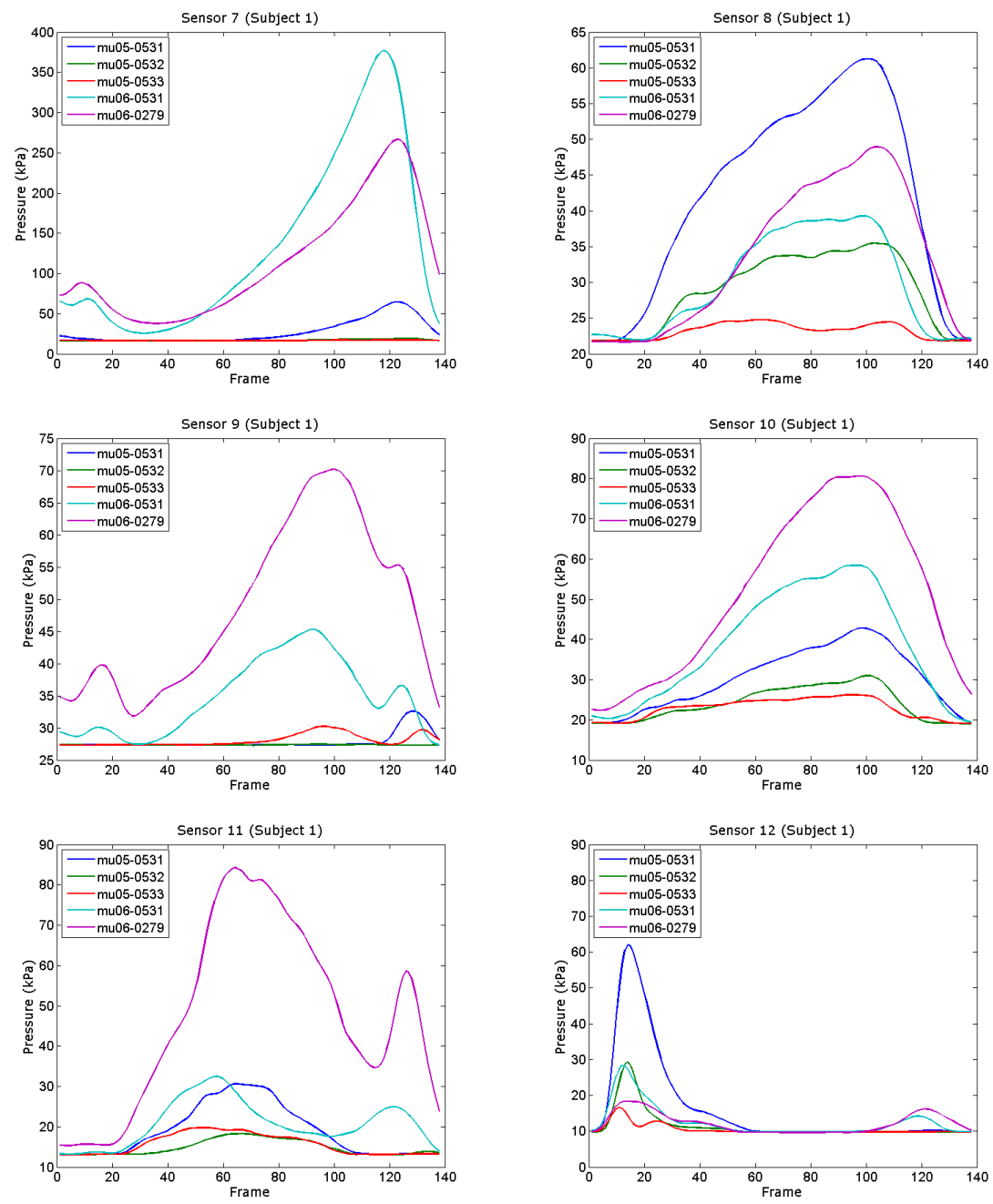

Figure 4.9: Dorsal pressure distribution for subject 1, sensor 7 to sensor 12. 

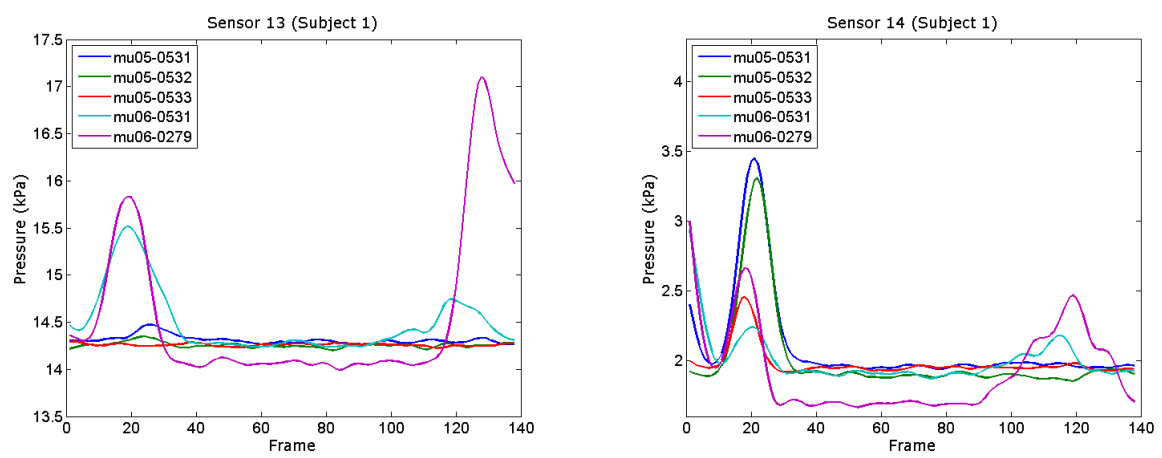

Figure 4.10: Dorsal pressure distribution for subject 1, sensors 13 and 14 .

it was necessary to analyze the pressure distribution on each anatomical point of the foot of the subject during a complete step. For each subject, a similar pattern of the pressure distribution was obtained for all the materials on each anatomical point. Fig. 4.8 to Fig. 4.10 show the pressure distribution on each sensor for one of the subjects, and Figs. 4.11 to 4.13 show the pressure distribution on each sensor for another different subject. Fig. 4.14 shows the pressure distribution on one of the sensors for each subject. Although some differences were found which could be due to little changes in the gait of the subject, the pressure curves for all the materials were similar for the majority of the sensors showing the tendency of that subject to walk in the same way using the same design of shoe.

\subsection{Discussion}

The curves of the pressure on the same foot anatomical point of a subject showed that effectively, for each subject, the gait cycle was similar 

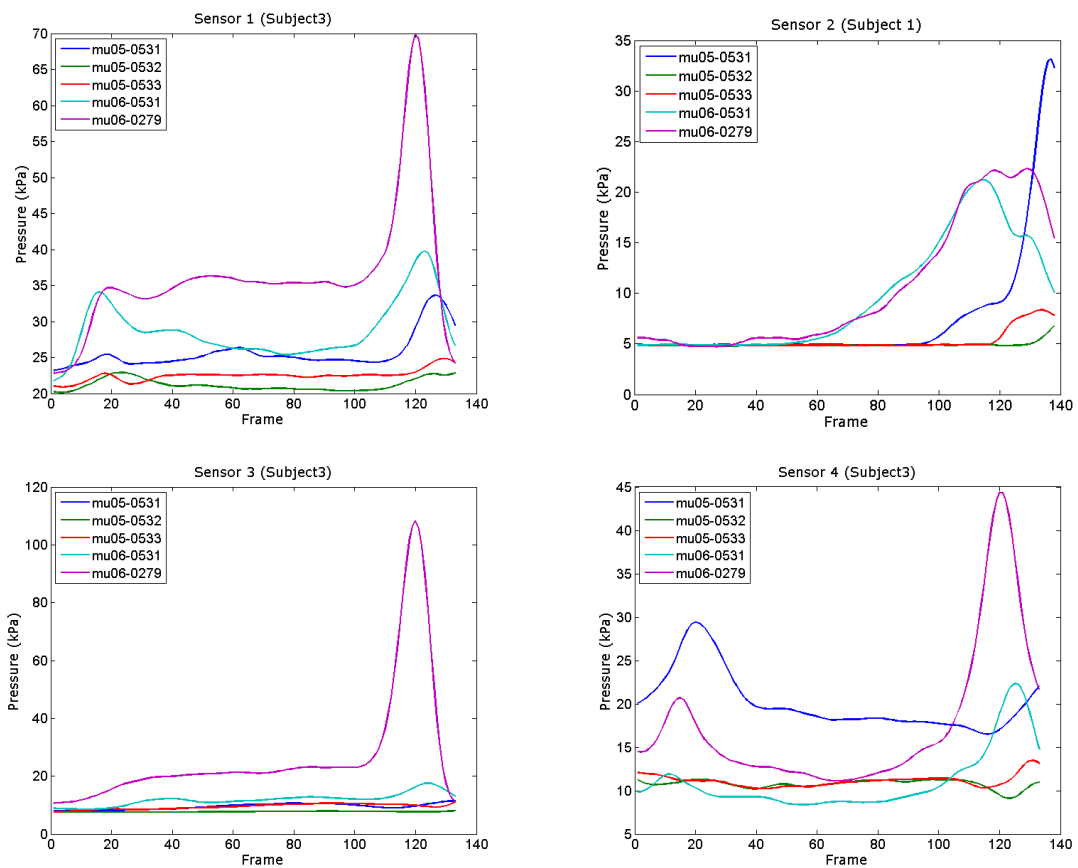

Figure 4.11: Dorsal pressure distribution for subject 3 on sensor 1 to sensor 4 . 

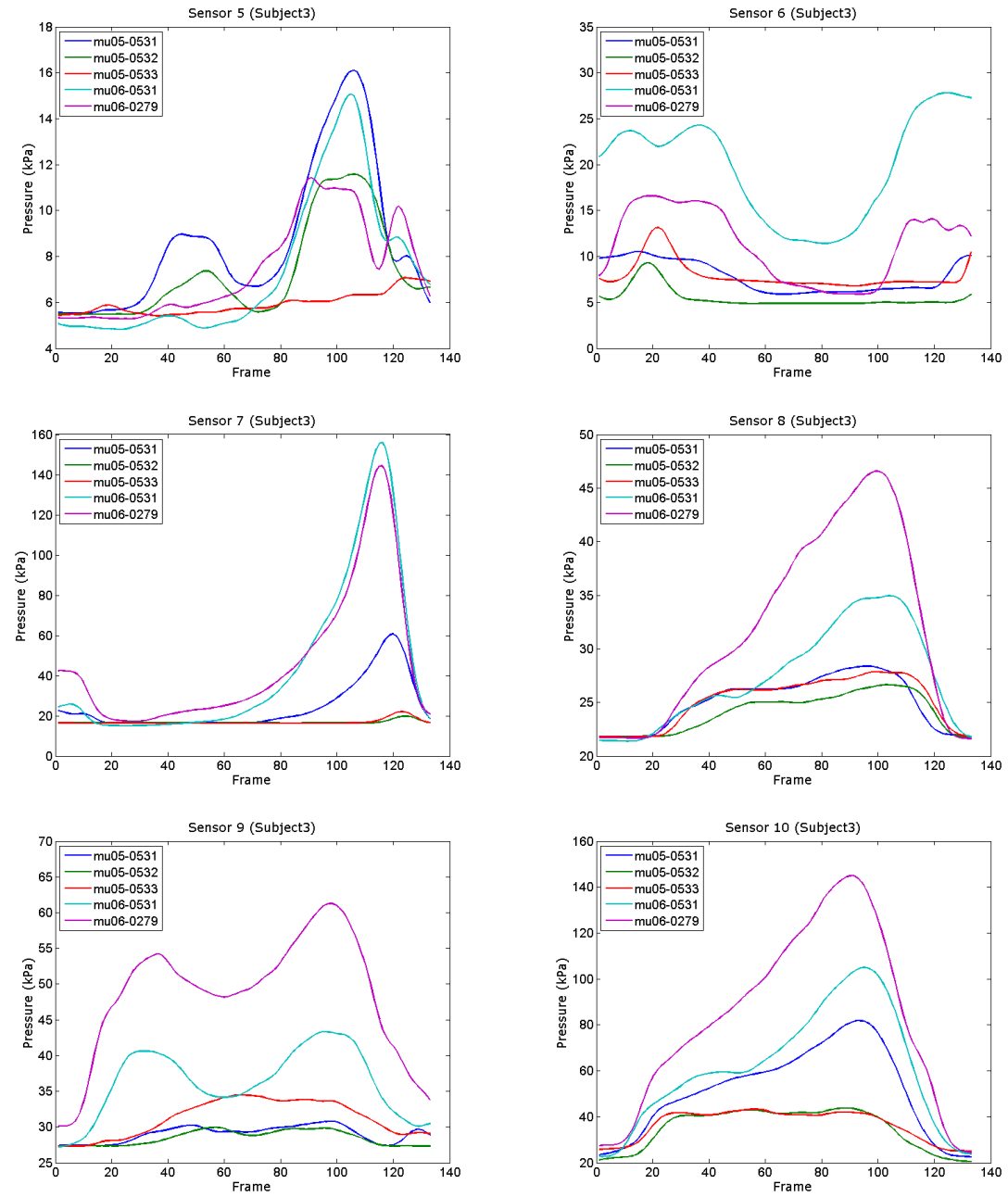

Figure 4.12: Dorsal pressure distribution for subject 3 sensor 5 to sensor 10. 

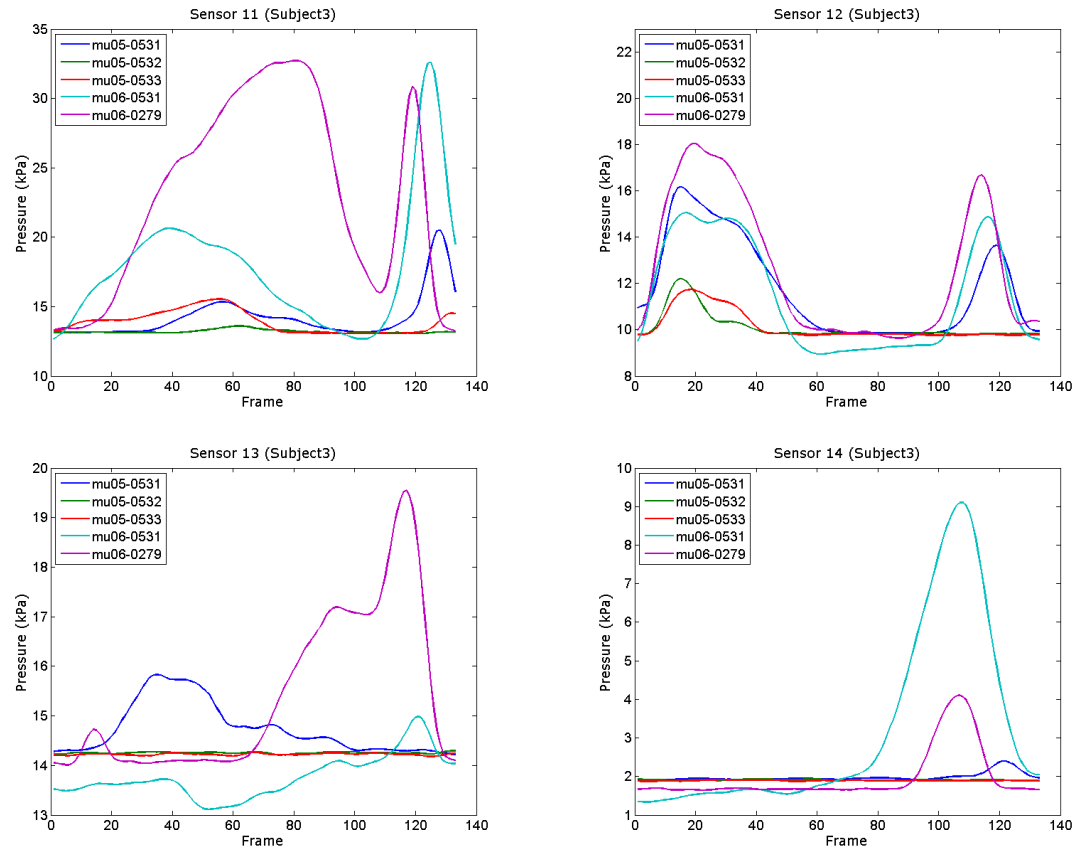

Figure 4.13: Dorsal pressure distribution for subject 3 sensor 11 to sensor 14 . 

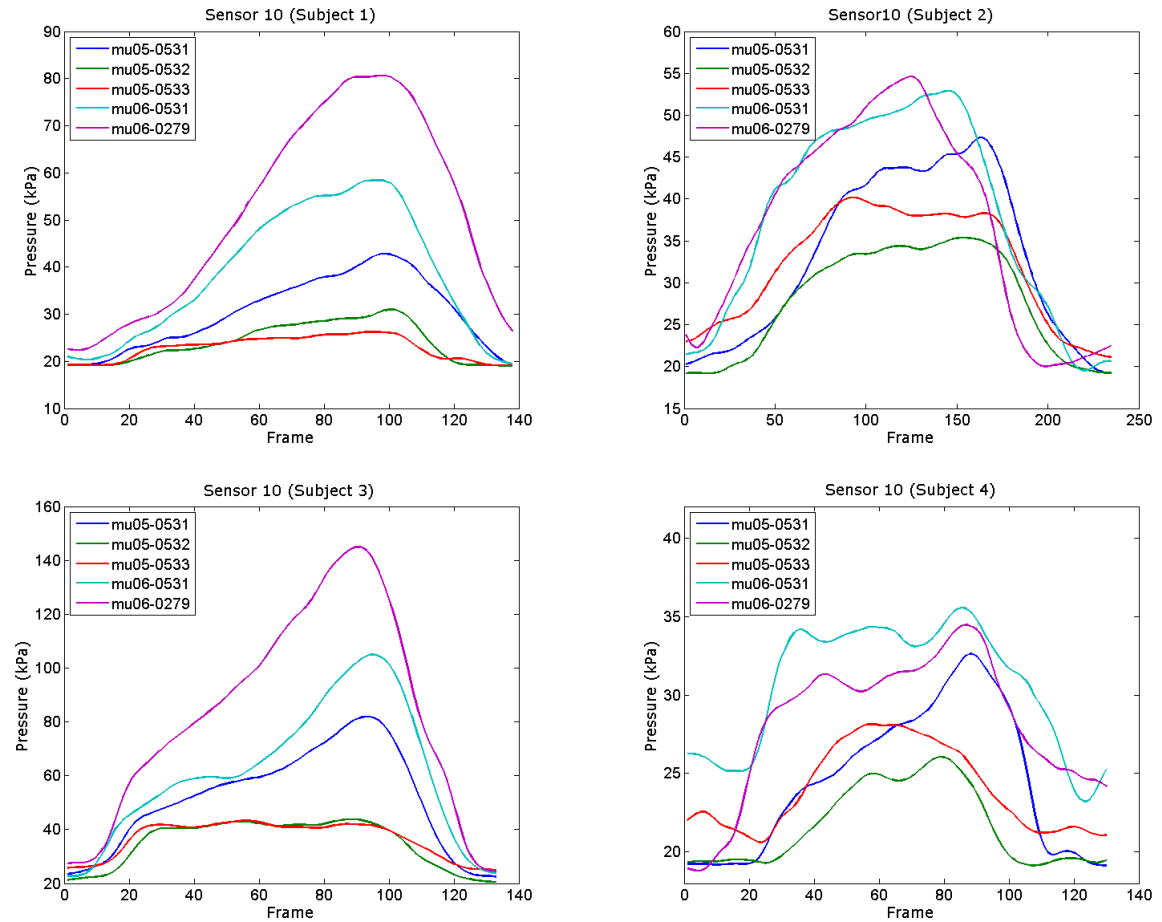

Figure 4.14: Dorsal pressure distributions on sensor 10 for each subject. 
wearing any of the shoes. This was corroborated with an analysis of crosscorrelation between the curves which provided correlation coefficient values above 0.85. Then, it is sufficient to use a single foot animation during a step to simulate a subject's gait cycle. Therefore, the animation of the foot reconstructed as described in Section 4.2 can be introduced in the virtual simulator as the generic gait of that individual [44].

The distributions of the pressures on each sensor located on the anatomical points of the foot of a subject are the basis of this chapter conclusions. On each sensor, the pressure distribution follows a similar pattern for a given individual. This suggests that the gait of a subject is similar wearing any shoe. Actually, there are some differences for any of the materials in the curves, but the pressure curves presented enough similarities to say that the gait of the user is similar. The fact is that, to the author's knowledge, there are no models that simulate the foot movement during a step due to its complex structure. This model has been constructed from the movement record of the main anatomical points of the foot. The main anatomical points were chosen by expert anatomists. These are bones or joints with behavior of rigid-body that will not change in gait wearing a shoe. Therefore, this model is a good approximation very useful for our purpose [44], which is to simulate the deformation of the shoe upper while walking.

\subsection{Summary}

It can be concluded that an animation of the foot that is reconstructed using the procedure presented in this thesis can be used as the generic gait for a subject in the footwear virtual simulator. With this kind of animation and with a more evolved FE implementation which includes large deforma- 
tions, Simucal will be able to provide a virtual simulation of the shoe-foot interaction while walking. Thus, the final evaluation of the prototype will be not only static, but also dynamic because it will be performed during a complete step. Future work will be devoted to measure the dorsal pressures for a given individual wearing other type of shoes. This will be carried out to confirm whether or not the gait of the subject is similar for any shoe to be worn. 
Chapter 4. Foot animation 


\section{Chapter 5}

\section{Behavior of the shoe upper material in gait}

The aim of the work developed in this chapter is to simulate the resistance to damage on lasting of some kinds of leathers used as shoe upper materials, as well as a shoe forming process using lasts. The framework of this work is the complete simulation of the footwear performance while walking, and these experiments have been chosen because they can model the deformation of the shoe upper materials in gait. The tests have been simulated using the Finite Element Method. In particular, the commercial software package ANSYSß has been used. The proposed model for the working range is a linear elastic orthotropic model. The results of the simulation show that, in most of the cases, the orthotropic model closely represents the real behavior of the leathers chosen for this work. 
Chapter 5. Behavior of the shoe upper material in gait

\subsection{Introduction}

To the author's knowledge, only one model that simulates the mechanical behavior of leather has been found in the literature. This model was proposed by Lin and Hayhurst in [104]. These authors proposed the expression shown in Eq. 5.1 for the strain energy density function applicable for the membrane state, where $e_{i j}$ are the components of the Lagrange (Green) strain tensor, and $J_{3}=e_{11} e_{22} e_{33}$. To simulate the shoe upper deformation, both membrane and bending behavior must be taken into account.

$$
\begin{gathered}
W=\frac{1}{2}\left(c_{11} e_{11}^{2}+c_{22} e_{22}^{2}+c_{33} e_{33}^{2}+c_{44} e_{12}^{2}\right)+ \\
c_{12} e_{11} e_{22}+c_{23} e_{22} e_{33}+c_{13} e_{11} e_{33}+c_{77} J_{3}
\end{gathered}
$$

The linear elastic model proposed in Chapter 3 of this thesis and already published in [43] considerers two behaviors for the shoe upper materials, membrane and bending. This model was developed to evaluate the deformation of the shoe upper at the initial stage, i.e., when individuals put their shoes on. To simulate this initial fit between the foot and the shoe, small strains were assumed. However, this cannot be assumed if a complete step needs to be simulated. In this case, large deformations must be considered in order to achieve more realistic simulations.

In this chapter, a new material model proposed for the simulation of the deformation of the shoe upper materials in gait is evaluated, an orthotropic model derived from the analysis of the tensile tests used for determining the elastic parameters of the leathers. The validation is carried out with the standard test ISO 17693:2004 used to measure the resistance of the leathers to damage on lasting, and with another experiment specifically designed 
5.2. Materials and methods

to simulate a shoe forming process using shoe lasts. These validations take into account large deformations and the Thin Shell Theory, which includes both, membrane and bending behavior.

As the foot surface has little soft tissue, its behavior can be assumed to be determined by the behavior of the bones that form it [10]. In this sense, the resistance to damage on lasting tests, which consist of applying a controlled force by means of a sphere on a sample of the material (Fig. 5.4), could simulate either a bone or a joint pushing the shoe upper, for example, the hallux. In the case of the simulation of the forming process with lasts, a cylindrical piece made of the same material of the shoe lasts is what pushes the sample of material (Fig. 5.7). Therefore, this experiment could model how the shoe upper material is pushed by the foot dorsum while walking. Thus, both tests can be used to validate the membrane and bending model proposed in chapter 3 .

Section 5.2 describes the experimental tests performed for the study of the leather behavior. Section 5.3 describes its simulation, and Section 5.4 analyzes the approximations assumed for the proposed model.

\subsection{Materials and methods}

The mechanical properties of the shoe upper leather are mainly dependent on composition and structure of the skin. To gain a broader appreciation of this it is necessary to examine the structure of the skins, Fig. 5.1, where $x_{1}$ and $x_{2}$ represent the directions parallel and perpendicular to the backbone respectively, and $x_{3}$ is the thickness direction. Fig. 5.1(a) shows the cross sections of the dead skin before tanning and rolling. The surface layer, known as epidermis, is a hard cellular material which protects the skin 
Chapter 5. Behavior of the shoe upper material in gait

from abrasion. The epidermis and surface hair are removed during tanning process. The layer below the epidermis is called the grain layer Fig. 5.1(b), which consists of very fine, numerous and densely packed fibre bundles. It becomes the upper surface of the finished leather, after removal of the epidermis and heavy rolling, which also reduces the leather thickness from $h_{0}$ to $h$. The part of the skin below the grain layer is called the corium and contains coarse bundles of collagen fibres and $1 \%$ of elastin fibres, which is the source of strength of leather [105] and varies greatly in thickness from one region to another [106]. The different fibres of the grain and corium layers are interwoven at the boundary line, making difficult to define the precise location of this interface. Mechanically, collagen fibres are strong in the direction of the fibre axes, and the elongation at failure is about $2 \%$ [89].

In the dead animal skin, Fig. 5.1(a), the fibres are set in a matrix of Natural Biding Agents (NBA), such as blood, etc. These agents are removed during the tanning process to create an open structure of fibres which is held together by the interwoven arrangement of the fibres. During the later stages of the tanning process the pores between the fibres are partially filled with oils and greases which determine the subsequent behavior of the leather. The partial filling of the zones between adjacent fibres produces a porous matrix structure. Under applied uniaxial loading, this type of structure enables the fibres to rotate and to slide relatively to each other. Fig. 5.1(b) shows the cross sections of the tanned and rolled leather. The thickness dimension decreases from $h_{0}$ to $h$ by heavy rolling and the fibres running along thickness direction become more parallel to the leather surface. The fibres are known to be oriented longitudinally, or parallel to the backbone, to different degrees depending upon the location on the skin. Fig. 5.1 also shows that the majority of fibres are oriented parallel to $x_{1}-x_{3}$ plane in the $x_{1}$ direction. This can be detected from differences in tensile 


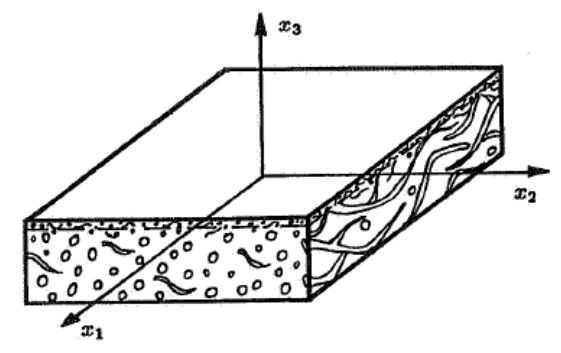

(a)

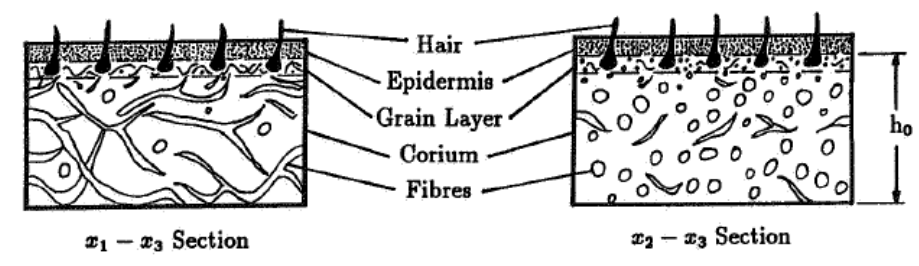

(b)

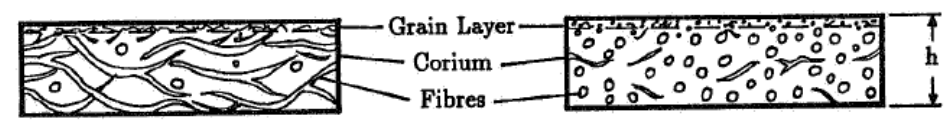

$x_{1}-x_{3}$ Section

$x_{2}-x_{3}$ Section

Figure 5.1: Schematic diagram of the structure of leather before (a) and after (b) tanning process. Figure from [104]. 
Chapter 5. Behavior of the shoe upper material in gait

strengths and strains at fracture for different directions [90]. The breaking load measured in any strength test is directly related to the number of fibres which become involved in the test prior to rupture. According to Roddy [105], the tensile stress is higher in the direction parallel to the backbone than in the direction perpendicular to it; as expected, the strains at failure have an inverse directional dependence.

Shoe upper leather when tested uniaxially has a non-linear stress-elongation relationship [107]. On initial monotonic loading for a typical test, the stress increases proportionally with elongation up to a level of $20 \%$. Subsequently, for large strains, the behavior is non-linear with stress reaching a plateau at failure with elongation in the region of $30-60 \%$. For elongation up to $20 \%$ the mechanism of deformation is due to fibre rotation. Above these elongation levels deformation takes place by the combined mechanism of fibre rotation and of slippage between adjacent fibres. This is consistent with the work of Mitton [90] who argued that the dominant strain mechanisms are fibre rotation and fibre network distortion, and no fibre elongation (the failure strain of a fibre being $2 \%$ ). Mitton [90] compared this behavior with that of a sheet of wire mesh with the difference that the fibre structure was three dimensional instead of two dimensional. When a tension stress was applied to the wire mesh net, an extension was produced which, was partly due to the elongation of the wires that formed the mesh, but which was mainly due to its change of shape (rotations).

Generally, shoe upper leather has been recognized as an orthotropic material by Mitton in [90] and Kanagy in [106]. They showed experimentally that the directions parallel and perpendicular to the backbone and the through-thickness directions may be defined as the principal directions of the leather. In addition, bi-axial experiments were carried out on rabbit skin by Lanir \& Fung in [108, 109], who verified that the mechanical properties of the skin are orthotropic. Hence, this imposes the form of 
5.2. Materials and methods

multi-axial constitutive equations required to model the behavior of the leather.

Monotonic loading tests were carried out at different strains rates by Maeser and by ASTM-ALCA Joint Committee [110] and the results showed a small dependence of the stress-strain curve on strain rate. It would therefore appear that over the wide strain rate range employed at room temperature there is not a strong time dependent effect. This was confirmed in relaxation of applied stress in specimens strained almost to failure. The stress relaxed by typically $10-15 \%$ of the initial values over a period of $10-$ 50 minutes. Maeser also showed in [110] that when a uniaxial specimen is loaded repeatedly between zero load and a constant strain amplitude, the same stress relaxation behavior is observed, and the stress-strain response becomes more linear with increasing number of cycles. Also, in cyclic loading tests carried out on rabbit skin at constant strain rates, Lanir \& Fung $[108,109]$ showed that the stress-strain relationship is essentially independent of strain rate. From these experimental studies it may be concluded that the behavior of leather at room temperature, may be considered to be time independent.

\subsubsection{Uniaxial tensile tests}

Two pieces of calfskin were used in this work. We used calfskin because it was the kind of leather used by Lin \& Hayhurst in their work [104]. Six samples from each one of the skins of the same size and characteristics that those used by these authors (Fig. 5.2) were taken. Uniaxial tensile tests under similar conditions were performed. These conditions were:

1. The whole skins were screen printed with a grid with lines of $0.2 \pm$ $0.1 \mathrm{~mm}$ of thickness, so that the lines were parallel and perpendicular 
Chapter 5. Behavior of the shoe upper material in gait

to the backbone.

2. Six samples were cut from each skin piece parallel and perpendicular to the backbone from three areas: shoulders, butt, and belly (Fig. 5.3, left).

3. To perform the tensile tests, a universal testing machine INSTRON 3366 from INSTRON® (Fig. 5.3, right) was used. The same loading velocity and load cell used in [104], $4.5 \mathrm{~mm} / \mathrm{min}$ and $500 \mathrm{~N}$ respectively, were fixed for all the tests.

4. The machine was programmed to be stopped each $5 \mathrm{~mm}$ (as in [104]) during 30 seconds. In each stop, a photo of the sample was taken with a reflex camera Canon PowerShot SX20 IS placed at $1.5 \mathrm{~m}$ from the sample. The setup of the camera was focal length $300 \mathrm{~mm}$, aperture $\mathrm{f} / 5$, shutter speed $=1 / 4 \mathrm{~s}$, ISO $=100$, and focusing $=1.5 \mathrm{~m}$.

The tests were carried out at the footwear institute INESCOP, Instituto tecnológico del calzado y conexas (http://www.inescop.es/).

\subsubsection{Resistance to damage on lasting}

From the same areas of the skin, shoulders, butt and belly, circular samples of $45 \mathrm{~mm}$ of diameter were also cut in order to perform the tests of resistance to damage following the standard test ISO 17693:2004 (Fig. 5.4). These tests were also carried out at INESCOP.

To simulate the tests of resistance to damage on lasting, the Finite Element Method was used. In particular, the simulation was performed with the commercial software package ANSYS v12.0 (Fig. 5.5). 


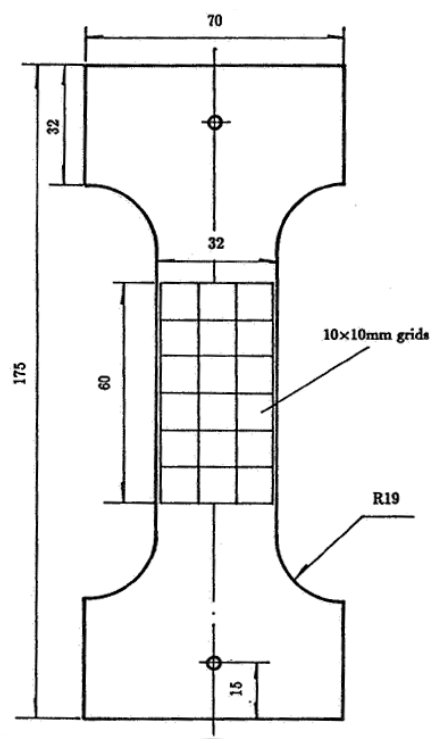

Figure 5.2: Shape and dimensions of uniaxial tensile test specimen. Dimensions in $\mathrm{mm}$ (figure from [104]).
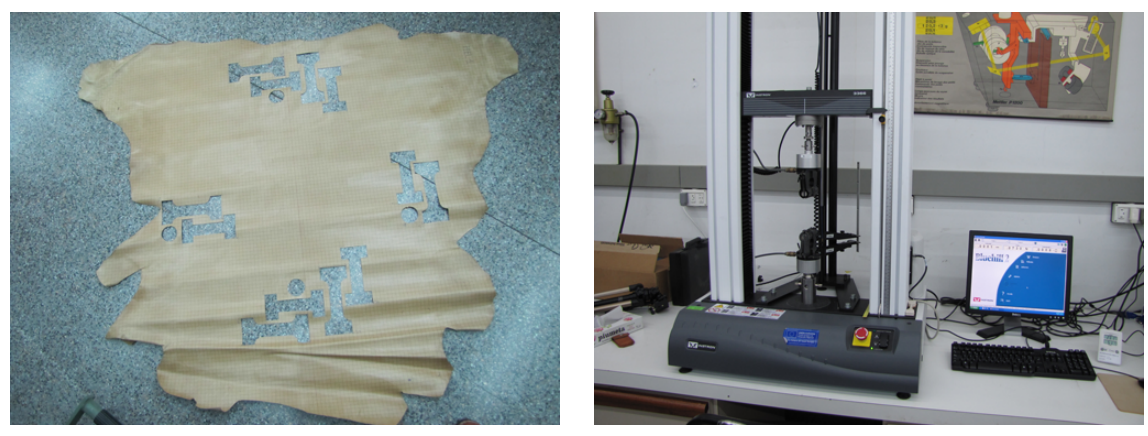

Figure 5.3: Calfskin (left). Universal testing machine (right). 
Chapter 5. Behavior of the shoe upper material in gait
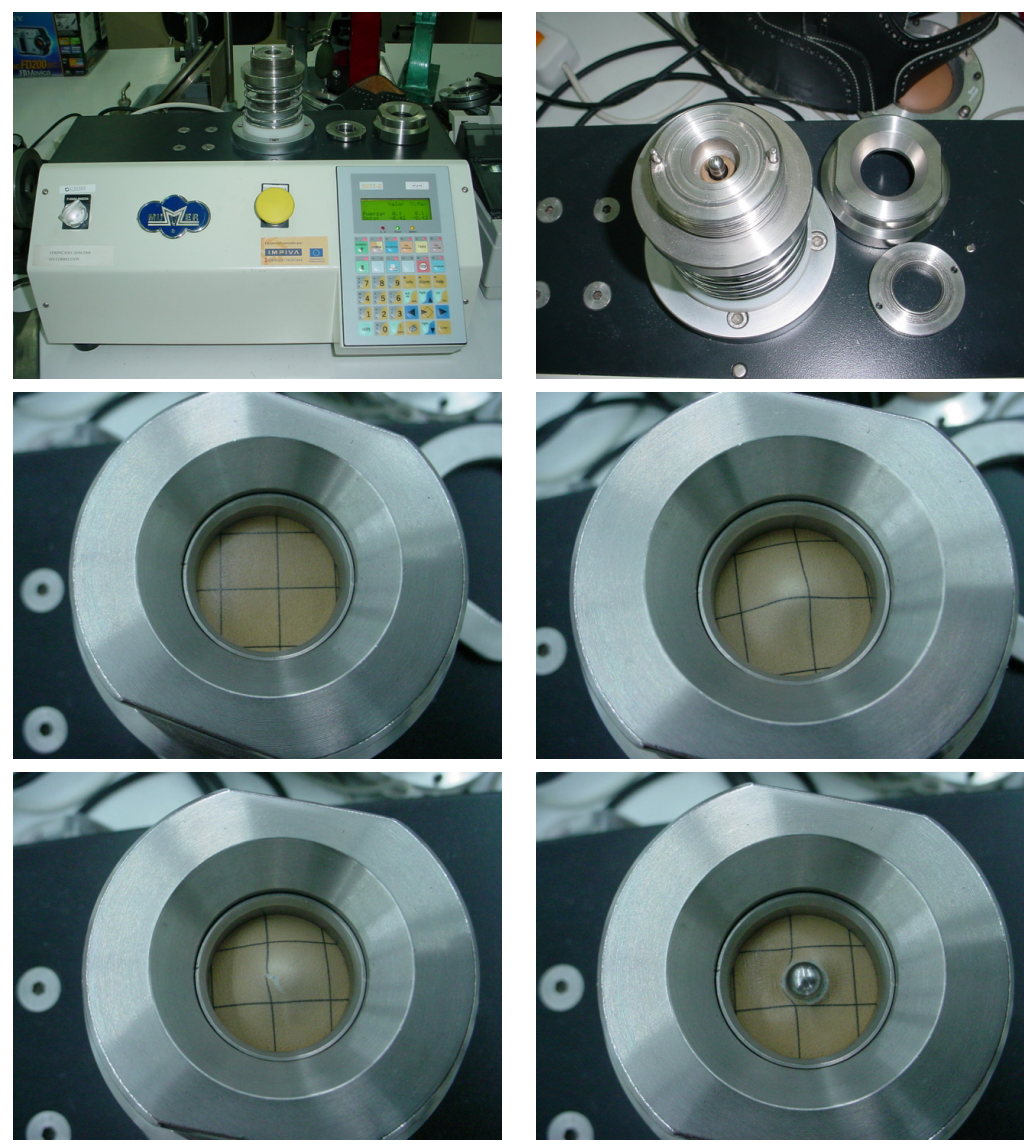

Figure 5.4: Test to measure the resistance to damage on lasting. 
The leather was meshed with shell elements (SHELL281). The mesh had 568 elements and 1185 nodes. It was refined at the center, where the contact was expected.
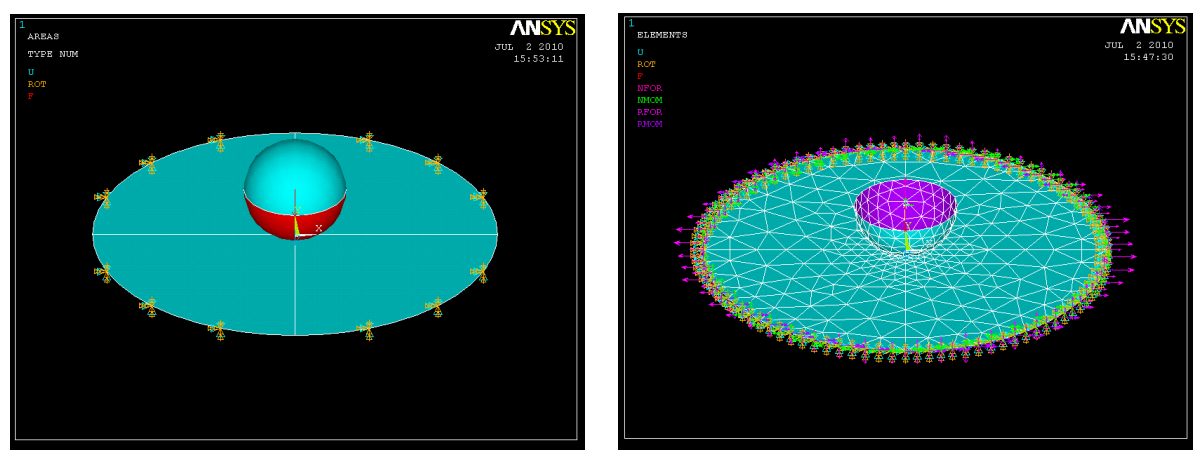

Figure 5.5: Simulation of the resistance to damage on lasting using ANSYS.

\subsubsection{Simulation of the shoe forming process using lasts}

To simulate how the shoe upper is deformed in a shoe forming process using lasts, Lin and Hayhurst designed a specific experiment in [111]. This experiment consisted of a compression test using a cylindrical piece made of a shoe last material that pushed a strip of material. Based on this experiment, a similar compression test was designed by INESCOP. A cylindrical last with radius of $40 \mathrm{~mm}$ and width of $45 \mathrm{~mm}$ was specially manufactured for this experiment. The chosen material was polypropylene (PP) a common material used for making shoe lasts. Six samples of the size and characteristics shown in Fig. 5.6 were cut from the shoulders, butt and belly of the same two skins used for the previous experiments. These samples were fixed between two serrated steel plates specially designed to 
be adapted to the universal testing machine INSTRON 3366 (Fig. 5.7). The test was performed in the same conditions that that fixed in [111] a cross-head velocity of $2.5 \mathrm{~mm} / \mathrm{min}$ and a load cell of $800 \mathrm{~N}$.

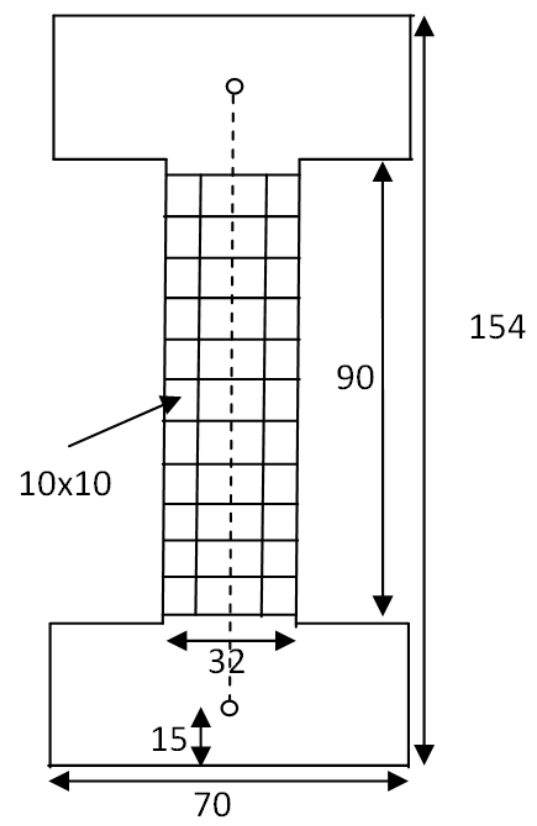

Figure 5.6: The shape and size of specimens for cylindrical last forming tests. All dimensions are in $\mathrm{mm}$.

To simulate the last forming tests, the commercial software package ANSYS v12.0 was used (Fig. 5.8). The leather was meshed with shell elements (SHELL281) and had 480 elements and 1025 nodes. Frictional contact conditions were imposed for the leather-last interface with a friction coefficient of 0.25 . This value was measured by Lin \& Hayhurst in [111]. 

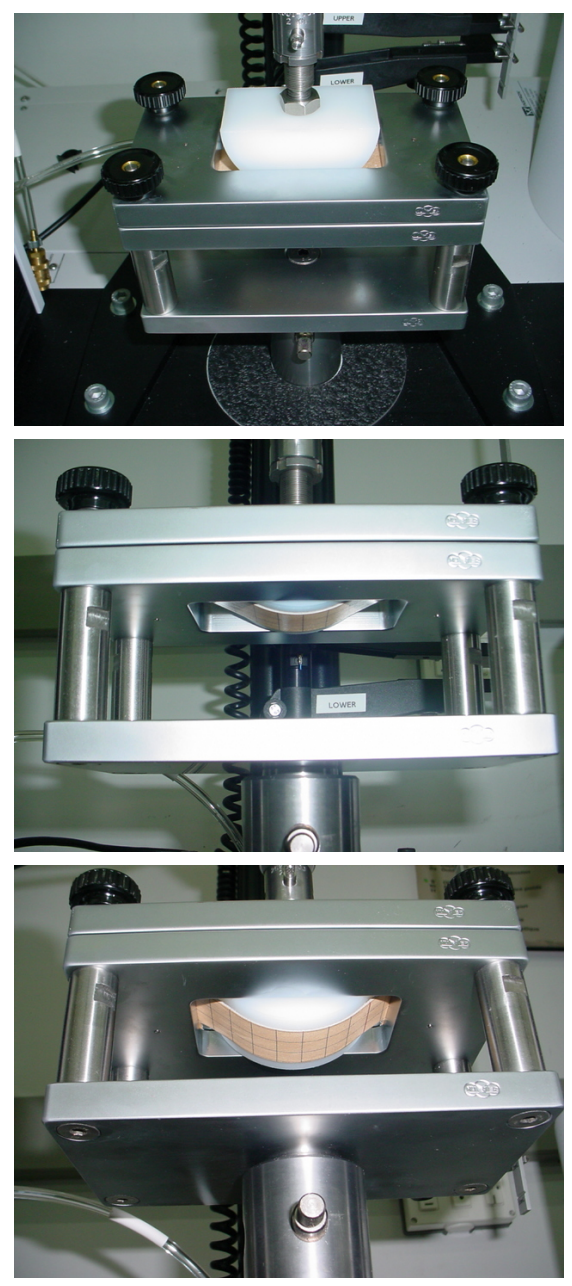

Figure 5.7: Simulation of the shoe forming process with shoe lasts. 
Chapter 5. Behavior of the shoe upper material in gait
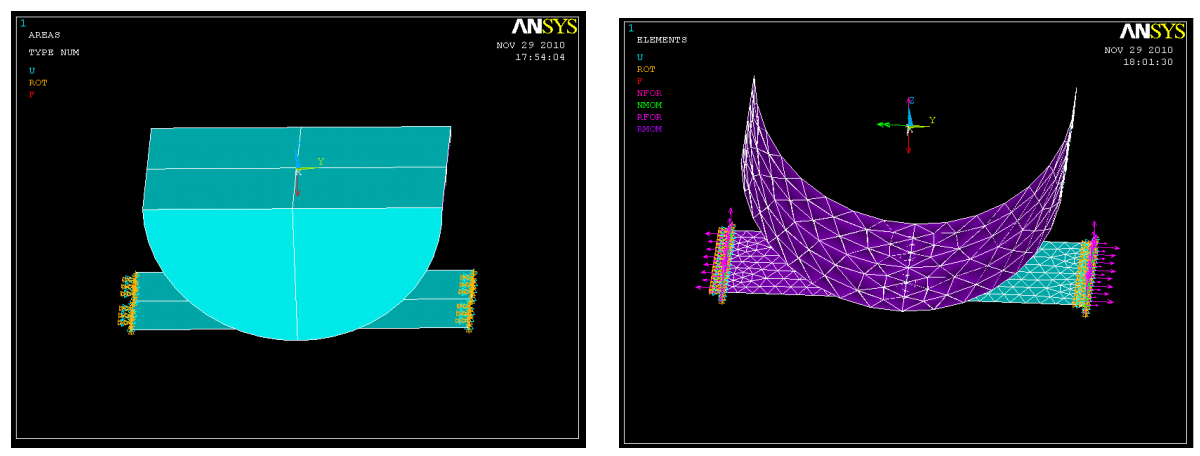

Figure 5.8: Simulation of the last forming test using ANSYS.

\subsection{Results and analysis}

\subsubsection{Uniaxial tensile tests}

The results of the uniaxial tensile tests for both skins are shown and analyzed in this section. For each area, shoulders, butt and belly, two graphs were plotted: tensile stress versus axial strain (Figs. 5.9 and 5.10), and lateral strain versus axial strain (Figs. 5.13 and 5.14).

\section{Tensile stress versus axial strain}

Figs. 5.9 and 5.10 show stress versus axial strain during the tensile test for the shoulder, butt and belly areas of the skins 1 and 2, respectively. This information was directly provided by the INSTRON software. The small peaks that can be observed pointing down in the graph mark the instants during which the machine was stopped (each $5 \mathrm{~mm}$ of axial elongation). 

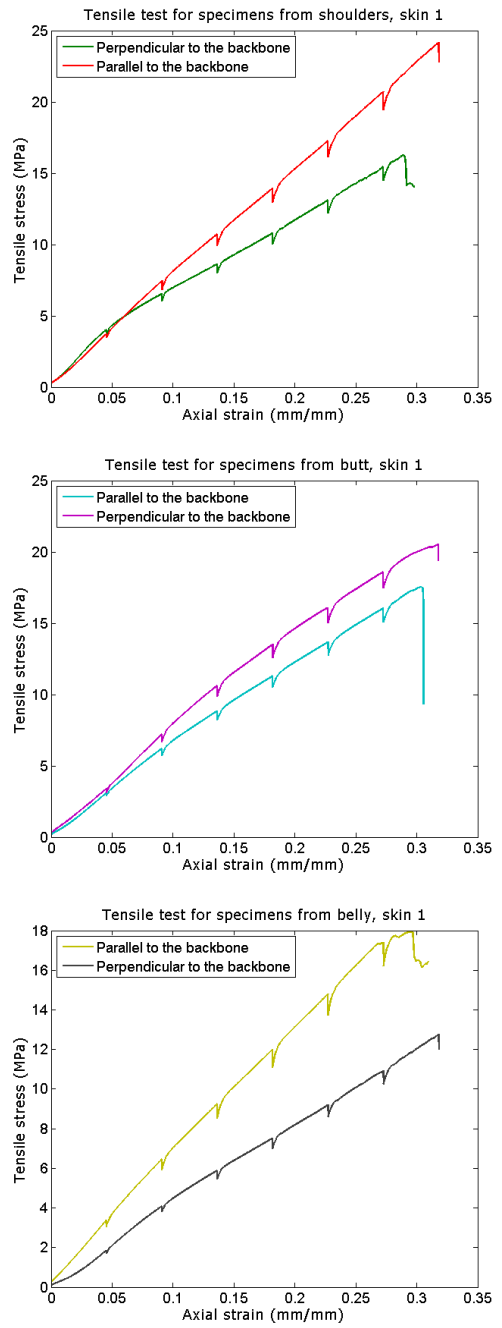

Figure 5.9: Tensile stress versus axial strain for shoulder (top), butt (middle), and belly (bottom) areas of skin 1 . 
Chapter 5. Behavior of the shoe upper material in gait
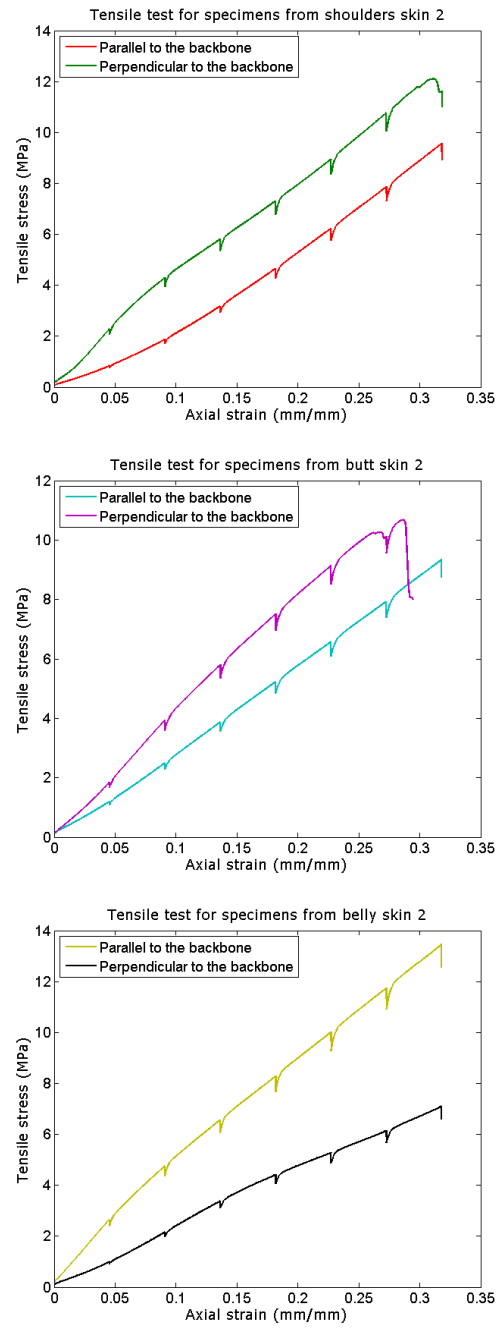

Figure 5.10: Tensile stress versus axial strain for shoulder (top), butt (middle), and belly (bottom) areas of skin 2. 
5.3. Results and analysis

The first data derived from the analysis of these graphs is that skin 1 has a greater strength than skin 2. The range in which the failure is produced for skin 1 is from 12 to $24 \mathrm{MPa}$ whilst for skin 2, it is from 7 to $14 \mathrm{MPa}$; this highlights the difference of strength between the skins. The result was expected since many authors have pointed out that these differences exist due to the differences between skins from animals of the same species.

The second data derived from the analysis is that not always the tensile stress is higher in the direction parallel to the backbone than in the direction perpendicular to it. According to Roddy [105], the tensile stress is higher in the direction parallel to the backbone than in the direction perpendicular to it; but, the strains at failure have an inverse directional dependence. Lin and Hayhurst confirmed these results with the single skin that they analyzed [104]. However, they obtained that the strain at the failure had not always inverse dependence. In our case, the results obtained in the butt area for skin 2 (Fig. 5.10, middle) contradict the affirmations of Roddy [105]. For the samples in this area, the stresses are higher in the direction perpendicular to the backbone than in the parallel direction, and the strain at failure is higher for the parallel direction than for the perpendicular. This same fact separately occurs in some of the other areas almost in $50 \%$ of the cases. For instance, the stresses are higher in the direction perpendicular to the backbone than in the parallel direction in the butt area for skin 1 (Fig. 5.9, middle), and in the butt and shoulder areas for skin 2 (Fig. 5.10, middle, and Fig. 5.10, top). Regarding the strain at failure, it is higher in the direction parallel to the backbone than in the perpendicular in the shoulder and belly areas for skin 1 (Fig. 5.9, top, and Fig. 5.9, bottom), and in the butt area for skin 2 (Fig. 5.10, middle). The possible explanation to these contradictory results is that our skins were different to those used in [104] and [105] as well as the techniques used for tanning them. Therefore, the formation and orientation of the bundles of 
Chapter 5. Behavior of the shoe upper material in gait
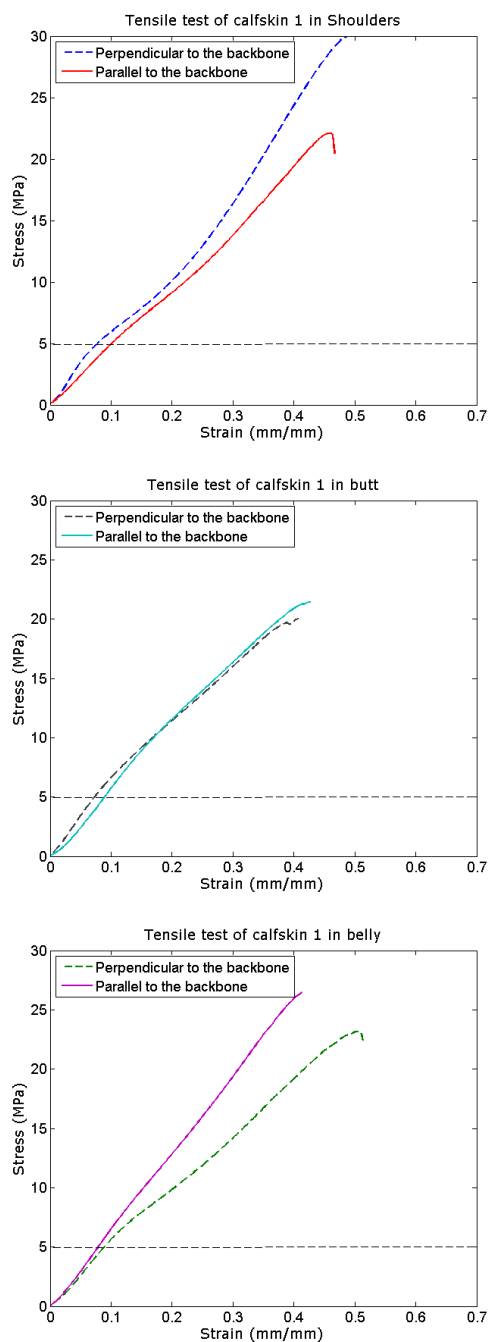

Figure 5.11: Stress versus strain from shoulder (top), butt (middle), and belly (bottom) areas of skin 1. Results of the tensile test following the standard UNEEN-ISO 3376 

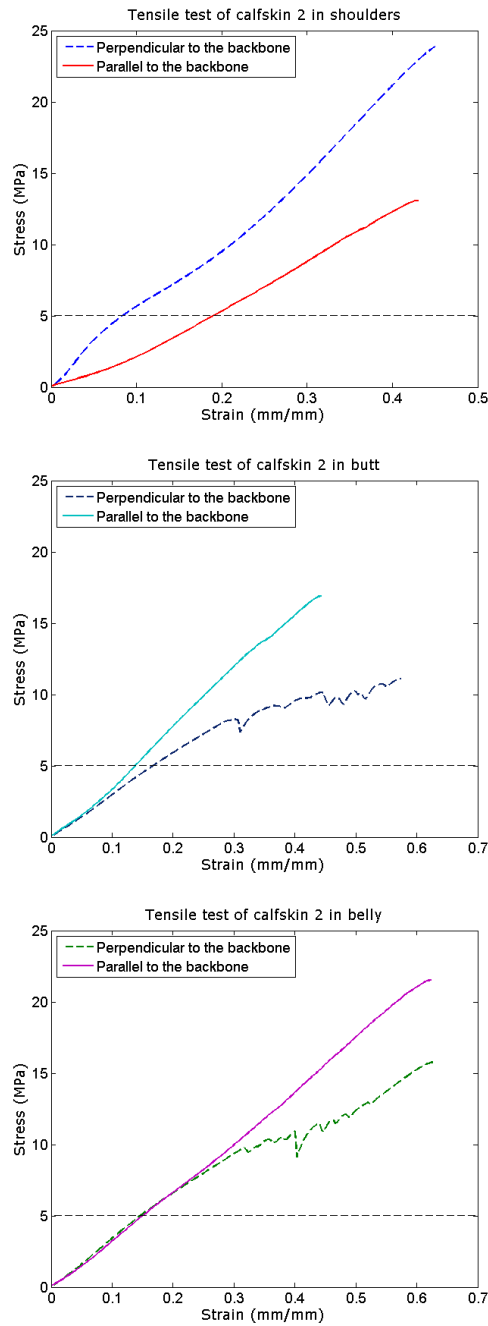

Figure 5.12: Stress versus strain from shoulder (top), butt (middle), and belly (bottom) areas of skin 2. Results of the tensile test following the standard UNEEN-ISO 3376 
Chapter 5. Behavior of the shoe upper material in gait

the collagen and elastin fibres, mainly responsible of the elastic behavior of the leathers [105], could be different to those used by these authors.

To confirm these results, tests according to the UNE-EN-ISO 3376 standard established for obtaining the elastic properties of the leathers were also carried out at INESCOP (although with samples cut in the shoulder, butt, and belly areas, in the direction parallel and perpendicular to the backbone). The main difference regarding the tests carried out following the proposal of Lin and Hayhurst is that the machine is not stopped during the elongation, the samples have different size, and they are cut throughout all the skin. As it can be observed in Figs. 5.11 and 5.12, the results are roughly the same and there is not a privileged direction in which the stress is higher than in the others. In fact, the curves obtained in the standard tests for the samples cut in the shoulder area of skin 1 (Fig. 5.11, top), and for the samples cut in butt area of skin 2 (Fig. 5.12 middle), present a inverse relationship between the stresses in the directions parallel and perpendicular to the backbone with regard to the stresses obtained in the curves for the tests based on the Lin \& Hayhurst experiment (Fig. 5.9 top, and Fig. 5.10 middle). Therefore, it is not possible to establish a conclusion about which direction, perpendicular or parallel to the backbone, presents more resistance. This could be due that the orientation of the fiber bundles in calfskins is a little more arbitrary than the previously established.

\section{Lateral strain versus axial elongation}

Figs. 5.13 and 5.14 show the lateral strain versus the axial elongation or displacement of the pincers for the shoulder, butt and belly areas of the skins 1 and 2 respectively.

To measure the lateral strain, a software application was developed. The 

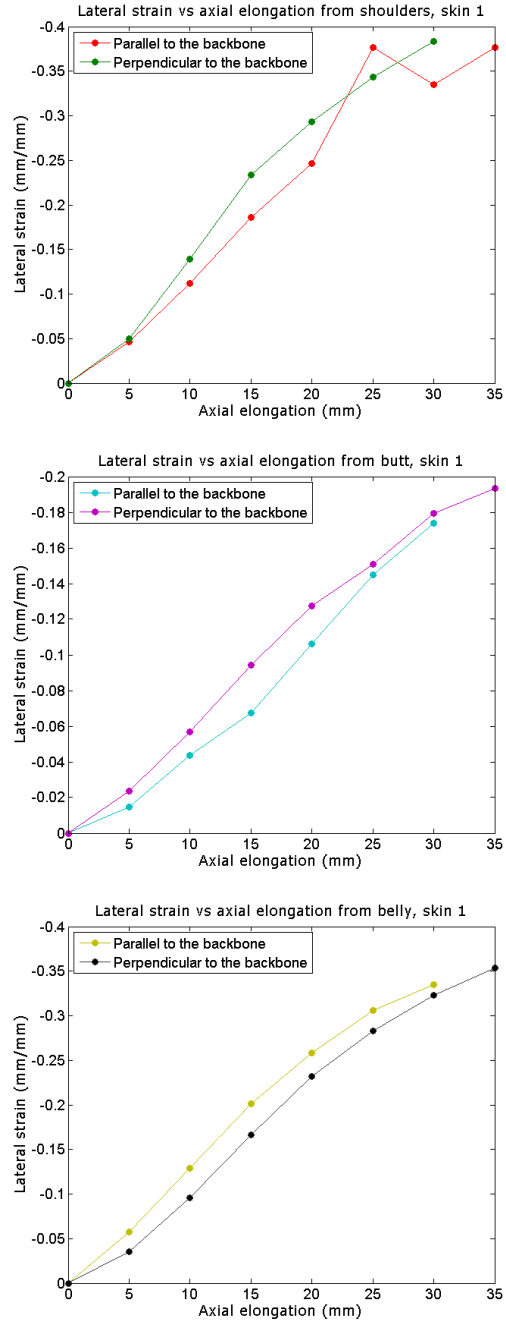

Figure 5.13: Lateral strain versus axial elongation for shoulder (top), butt (middle), and belly (bottom) areas of skin 1 . 
Chapter 5. Behavior of the shoe upper material in gait
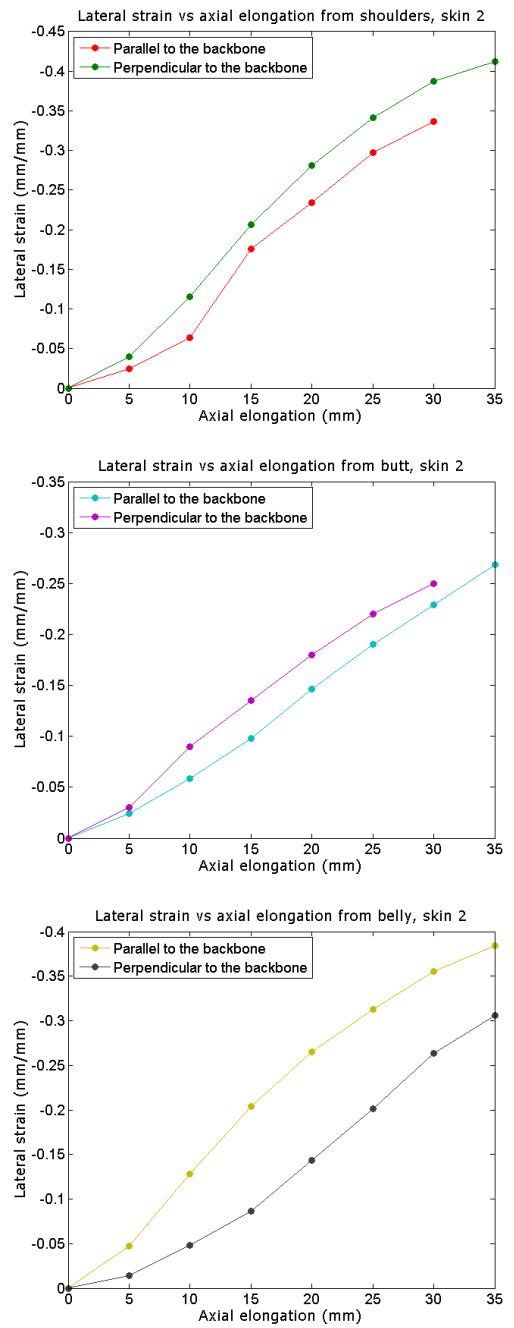

Figure 5.14: Lateral strain versus axial elongation for shoulder (top), butt (middle), and belly (bottom) areas of skin 2. 

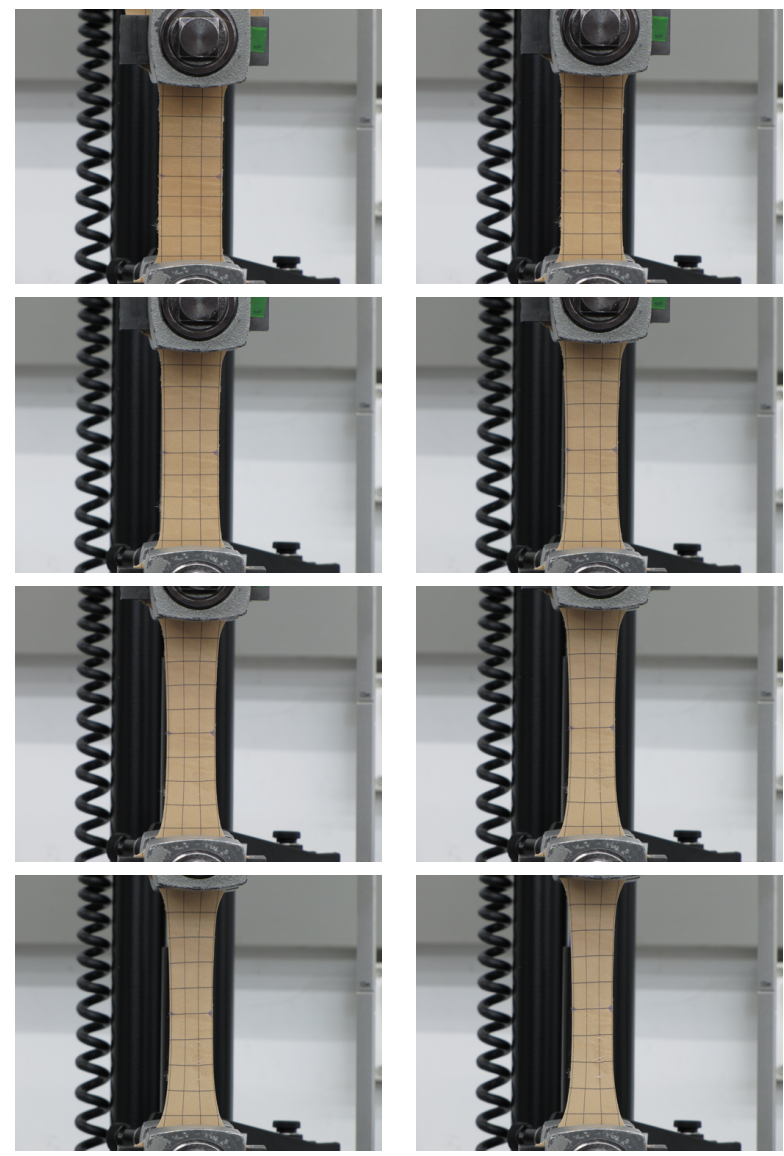

Figure 5.15: Deformation of the calfskin sample. 
Chapter 5. Behavior of the shoe upper material in gait

application was calibrated using the non-deformed sample, associating the distance between two pixels from the ends of the central grid square edge to $10 \mathrm{~mm}$. The pixels were marked picking directly on the the screen when this showed the image of the sample. A mean of the pixel distance was obtained from the central square four edges. Fig. 5.15 shows the tensile test images of a sample taken from the shoulder area of skin 1 .

For the next images of the deformed sample, the lateral strain was measured picking the pixels of the same square on the images of the deformed sample shown on the screen, and getting the mean distance between the four ends of this central square. The ratio between the lateral strain and the axial strain yields the Poisson's ratio (Eq. 5.2). The results for both skins are shown in Tables 5.2 and 5.5.

$$
\nu=-\frac{\varepsilon_{\text {lateral }}}{\varepsilon_{\text {axial }}}
$$

Summarizing, analyzing the data provided by the uniaxial tensile tests, both the tests performed following the Lin \& Hayhurst proposal and the tests performed in this thesis following the standard UNE-EN-ISO 3376, it can be established that the behavior of the leather is orthotropic as it was pointed out by Mitton in [90], Kanagy in [106], and by Lanir \& Fung in $[108,109]$.

As the maximum pressures on the foot surface are about $150-400 \mathrm{kPa}$ (Section 4.3, Chapter 4), the elongation of the leather for these values of the stress is under 20\%. Then, according to [104] the behavior of the leather can be assumed linear in this range. In fact, this behavior is confirmed by our data. Figs. 5.9, 5.10, 5.11, and 5.12 show a quasi-linear behavior; 
except for the samples cut in the perpendicular direction in the shoulder area for skins 1 and 2 (Figs. 5.11 and 5.12, top). Thus, the behavior of the leather is assumed linear and orthotropic for the numerical simulation of the resistance to damage on lasting and for the numerical simulation of the shoe forming using lasts carried out in this thesis. In addition, as up to $5 \mathrm{MPa}$ the stress-strain curves corresponding to the parallel and perpendicular to the backbone directions were very close each other, an isotropic linear elastic model was also proposed for these simulations.

\section{Materials with orthotropic behavior}

By definition, an orthotropic material has three orthogonal planes of symmetry regarding its material properties (Fig. 5.16). Such materials require nine independent variables (i.e., elastic constants) in their constitutive matrices [112]. The relationship between strain and stress for these materials is given by Eq. 5.3, which represents $\varepsilon=\mathbf{S} \sigma$, where $\mathbf{S}^{-1}$ is the stiffness matrix. The relationships shown in Eq. 5.4 are obtained from the symmetry of $\mathbf{S}$ [112].

$$
\left(\begin{array}{c}
\varepsilon_{1} \\
\varepsilon_{2} \\
\varepsilon_{3} \\
\gamma_{23} \\
\gamma_{31} \\
\gamma_{12}
\end{array}\right)=\left(\begin{array}{cccccc}
\frac{1}{E_{1}} & -\frac{\nu_{21}}{E_{2}} & -\frac{\nu_{31}}{E_{3}} & 0 & 0 & 0 \\
-\frac{\nu_{12}}{E_{1}} & \frac{1}{E_{2}} & -\frac{\nu_{32}}{E_{3}} & 0 & 0 & 0 \\
-\frac{\nu_{13}}{E_{1}} & -\frac{\nu_{23}}{E_{2}} & \frac{1}{E_{3}} & 0 & 0 & 0 \\
0 & 0 & 0 & \frac{1}{G_{23}} & 0 & 0 \\
0 & 0 & 0 & 0 & \frac{1}{G_{31}} & 0 \\
0 & 0 & 0 & 0 & 0 & \frac{1}{G_{12}}
\end{array}\right)\left(\begin{array}{c}
\sigma_{1} \\
\sigma_{2} \\
\sigma_{3} \\
\tau_{23} \\
\tau_{31} \\
\tau_{12}
\end{array}\right)
$$

Thus, the independent parameters that define the constitutive equations 
of a material with orthotropic behavior are nine, and they could be the Young's moduli in its three main directions: $E_{1}, E_{2}$, and $E_{3}$; the three Poisson's ratios: $\nu_{12}, \nu_{23}$, and $\nu_{31}$; and the three shear moduli: $G_{12}, G_{23}$, and $G_{31}$.

$$
\frac{\nu_{12}}{E_{1}}=\frac{\nu_{21}}{E_{2}} \quad \frac{\nu_{13}}{E_{1}}=\frac{\nu_{31}}{E_{3}} \quad \frac{\nu_{23}}{E_{2}}=\frac{\nu_{32}}{E_{3}}
$$

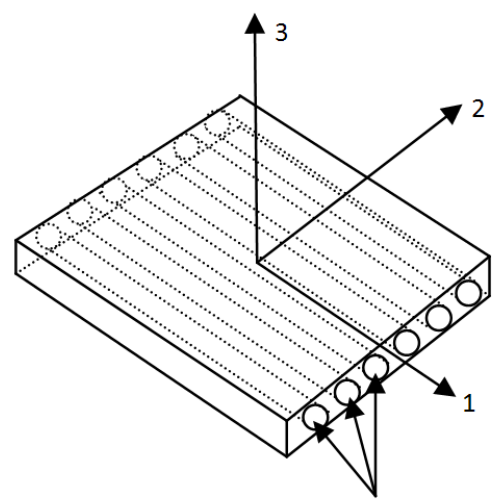

Fibers

Figure 5.16: Example of orthotropic material.

\section{Parameters of the simulation}

After the tanning process, the leather presents similar structure to orthotropic materials since the majority of the bundles of collagenous fiber are oriented in the direction parallel to the backbone (Section 5.2). Therefore, an approximation to a orthotropic behavior was assumed for the leather to estimate the Young's moduli and the Poisson's ratios. However, 
5.3. Results and analysis

due to the lack of the appropriate devices and standard tests to measure the shear moduli of the leathers $\left(G_{12}, G_{23}\right.$, and $\left.G_{13}\right)$, an average value of the isotropic estimations was assumed for them.

Two Young's moduli were obtained from a linear fitting from the stressstrain curve from the standard tests up to $5 \mathrm{MPa}$, one for the parallel direction and the other for the perpendicular direction. The highest Young's modulus was associated with the longitudinal direction $x_{1}$ since mechanically, collagen fibres are stiffer in the direction of the fibre axes [89], and the smallest value was associated with the transversal directions $x_{2}$ and $x_{3}$.

Regarding the Poisson's ratios, the Poisson's ratio $\nu_{12}$ was determined in the plane that contained both parallel and perpendicular directions. Its value was determined by the ratio between the lateral strain and the axial strain measured on the central square of the deformed samples, subjected to axial load, using the developed application for the tests based on the Lin \& Hayhurst experiment (Eq. 5.2). A linear fitting between the lateral strain and the longitudinal strain for the edges of the central square was developed to obtain this value. Considering the values obtained for the perpendicular sample and the parallel sample, which were very close, the value with the best correlation coefficient was chosen as major Poisson's ratio. Due to the lack of appropriate devices and standard tests to measure the relationship between the strain in the others directions, a value of 0.3 was assigned to the other two Poisson's ratio.

The orthotropic linear parameters for the two calfskins used in the experiments are shown in Tables 5.1 to 5.6, where 1 and 2 represent either the directions parallel and perpendicular to the backbone; 1 was assigned to the greatest value of the two Young's moduli obtained in these directions and 2 to the other one. 
Chapter 5. Behavior of the shoe upper material in gait

Table 5.1: Young's moduli for calfskin 1.

\begin{tabular}{lccc}
\hline calfskin1 & $E_{1}(\mathrm{MPa})$ & $E_{2}(\mathrm{MPa})$ & $E_{3}=E_{2}(\mathrm{MPa})$ \\
\hline Shoulders & $67.36, r^{2}=0.9945$ & $49.97, r^{2}=0.9991$ & 49.97 \\
Belly & $57.79, r^{2}=0.9941$ & $56.86, r^{2}=0.9927$ & 56.86 \\
Butt & $69.89, r^{2}=0.9983$ & $57.26, r^{2}=0.9902$ & 57.26 \\
\hline
\end{tabular}

Table 5.2: Poisson's ratios for calfskin 1.

\begin{tabular}{lccc}
\hline calfskin1 & $\nu_{12}$ & $\nu_{23}$ & $\nu_{31}$ \\
\hline Shoulders & $0.83, r^{2}=0.9978$ & 0.3 & 0.3 \\
Belly & $0.72, r^{2}=0.9993$ & 0.3 & 0.3 \\
Butt & 0.78 & 0.3 & 0.3 \\
\hline
\end{tabular}

Table 5.3: Shear moduli for calfskin 1.

\begin{tabular}{lccc}
\hline calfskin1 & $G_{12}(\mathrm{MPa})$ & $G_{23}(\mathrm{MPa})$ & $G_{13}(\mathrm{MPa})$ \\
\hline Shoulders & 15.98 & 19.21 & 22.56 \\
Belly & 16.67 & 21.87 & 22.05 \\
Butt & 17.78 & 22.02 & 24.45 \\
\hline
\end{tabular}

Table 5.4: Young's moduli for calfskin 2.

\begin{tabular}{lccc}
\hline \multicolumn{1}{c}{ calfskin2 } & $E_{1}(\mathbf{M P a})$ & $E_{2}(\mathbf{M P a})$ & $E_{3}=E_{2}(\mathbf{M P a})$ \\
\hline Shoulders & $62.17, r^{2}=0.9935$ & $26.19, r^{2}=0.9853$ & 26.19 \\
Belly & $34.91, r^{2}=0.9994$ & $33.64, r^{2}=0.9994$ & 33.64 \\
Butt & $35.48, r^{2}=0.9934$ & $30.34, r^{2}=0.9994$ & 30.34 \\
\hline
\end{tabular}

As the results from Tables 5.1 to 5.6 show, the Young's moduli were obtained with a very good correlation coefficient regarding the linear fitting confirming the assumption of linear behavior below 5MPa. The Poisson's 
Table 5.5: Poisson's ratios for calfskin 2.

\begin{tabular}{lccc}
\hline calfskin2 & $\nu_{12}$ & $\nu_{23}$ & $\nu_{31}$ \\
\hline Shoulders & $0.88, r^{2}=0.9833$ & 0.3 & 0.3 \\
Belly & $0.60, r^{2}=0.9944$ & 0.3 & 0.3 \\
Butt & 0.77 & 0.3 & 0.3 \\
\hline
\end{tabular}

Table 5.6: Shear moduli for calfskin 2.

\begin{tabular}{lccc}
\hline calfskin2 & $G_{12}(\mathbf{M P a})$ & $G_{23}(\mathbf{M P a})$ & $G_{13}(\mathbf{M P a})$ \\
\hline Shoulders & 13.22 & 10.07 & 16.99 \\
Belly & 9.93 & 12.94 & 13.18 \\
Butt & 9.27 & 11.67 & 12.66 \\
\hline
\end{tabular}

ratio correlations were also good for the shoulder and belly area. Taking into account that the butt area of both skins was very wrinkled (Fig. 5.3, left), the Poisson's ratios at this area had to be estimated as the mean between the ratios obtained for the other two areas.

In the case of the linear isotropic model, only two parameters are needed: The Young's modulus, which was estimated as the mean between the moduli obtained for the two directions (perpendicular and parallel), and the Poisson's ratio, whose value was assumed to be the highest value permitted for an isotropic model which is 0.5 (i.e., incompressible material). For two material behaviors (orthotropic and isotropic), the simulation was performed with ANSYS v12.

\subsubsection{Resistance to damage on lasting}

Fig. 5.17 shows the results of the resistance to damage on lasting for both skins (top) compared with the results of the tensile tests (bottom) already 
Chapter 5. Behavior of the shoe upper material in gait

shown in Figs. 5.11 and 5.12. In the case of the skin 1, the results of the resistance to damage on lasting are very close for all the areas (Fig. 5.17, top left). This is also what happens with the results of the tensile test below $5 \mathrm{MPa}$ (Fig. 5.17, bottom left). In fact, this is the range of pressure reached in this experiment since the maximum force was about $100 \mathrm{~N}$ and the diameter of the sphere is $25 \mathrm{~mm}$ (ISO 17693:2004). This also agrees with the Young's modulus values obtained for this skin in the three areas (Table 5.1), which are very close. Thus, a simulation of the leather behavior in these tests could be represented by a single average model of the behavior in all the areas for this case. However, in the case of skin 2, the behavior of the leather in the shoulder area (Fig. 5.17, top right) is not so close to the behavior in the other areas, a behavior that also agrees with the tensile tests below $5 \mathrm{MPa}$ (Fig. 5.17, bottom right), and with the values of the Young's moduli obtained for these areas (Table 5.4). The value of the Young's modulus for the shoulder area $(62.17 \mathrm{MPa})$ is far from the values in the other two areas $(34.91 \mathrm{MPa}$ for belly area and $35.48 \mathrm{MPa}$ for butt area). So, a specific set of parameters has to be used to model the leather behavior in each area.

The resistance to damage on lasting was simulated with ANSYS v12. Fig. 5.18 shows an example of the simulation for the shoulder area of skin 1. From the results provided by ANSYS, the force exerted by the sphere versus the displacement of the central point of the shell was measured and it is shown in Figs. 5.19 and 5.20. A load of $100 \mathrm{~N}$ was applied to the sphere. This value was chosen to be sure that it was under of the failure load.

Looking at Figs. 5.19 and 5.20, the results show that the orthotropic behavior closely represents the real behavior of the leather except in the belly area, which is better represented by the linear model for both skins (Figs. 5.19 and 5.20, bottom), although the trend of the curve is better 
5.3. Results and analysis
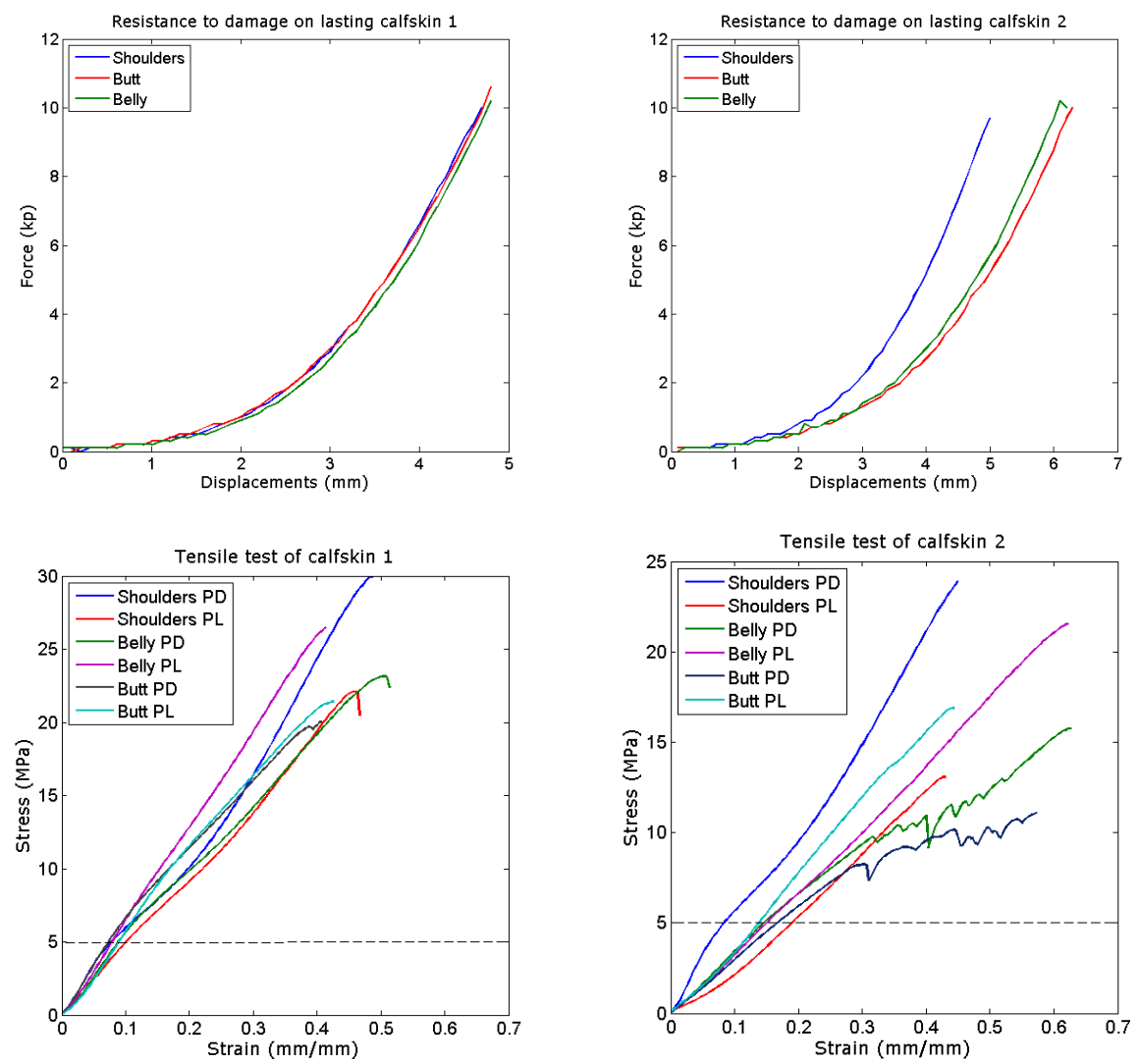

Figure 5.17: Load versus displacement of the central point of the samples cut in shoulder, butt and belly areas of skin 1 (top left) and of skin 2 (top right). Stress versus strain for the shoulder, butt and belly areas of skin 1 (bottom left) and of skin 2 (bottom right). PD and PL mean perpendicular and parallel to the backbone respectively. 
Chapter 5. Behavior of the shoe upper material in gait
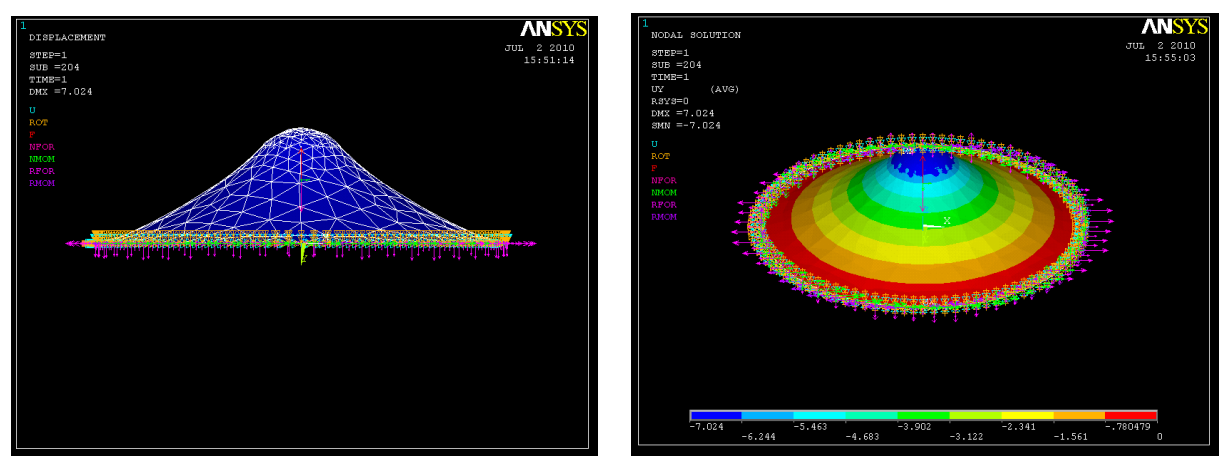

Figure 5.18: Simulation of the resistance to damage on lasting using ANSYS for the shoulder area of skin 1. Deformed mesh (left) and displacements (right).

represented by the orthotropic model. In fact, in all the cases, the trend is better represented by the orthotropic model than by the isotropic model. For the samples cut in the shoulder areas of skin 2 (Fig. 5.20, top), the orthotropic model does not represent so accurately the real behavior of the leather as in the other areas. This is due to the behavior in the perpendicular direction is less linear than the rest (Fig. 5.17, bottom right).

\subsubsection{Last forming tests}

Taking into account that the linear orthotropic model is the model that best represents the leather behavior in the simulation of the resistance to damage on lasting tests, this model was chosen to simulate the shoe forming process using lasts. These tests were simulated in ANSYS v12 (Figs. 5.21) and the results of the simulation are compared with the experimental results in Figs. 5.22 and 5.23.

Figs. 5.22 and 5.23 show how the linear orthotropic model can represent 

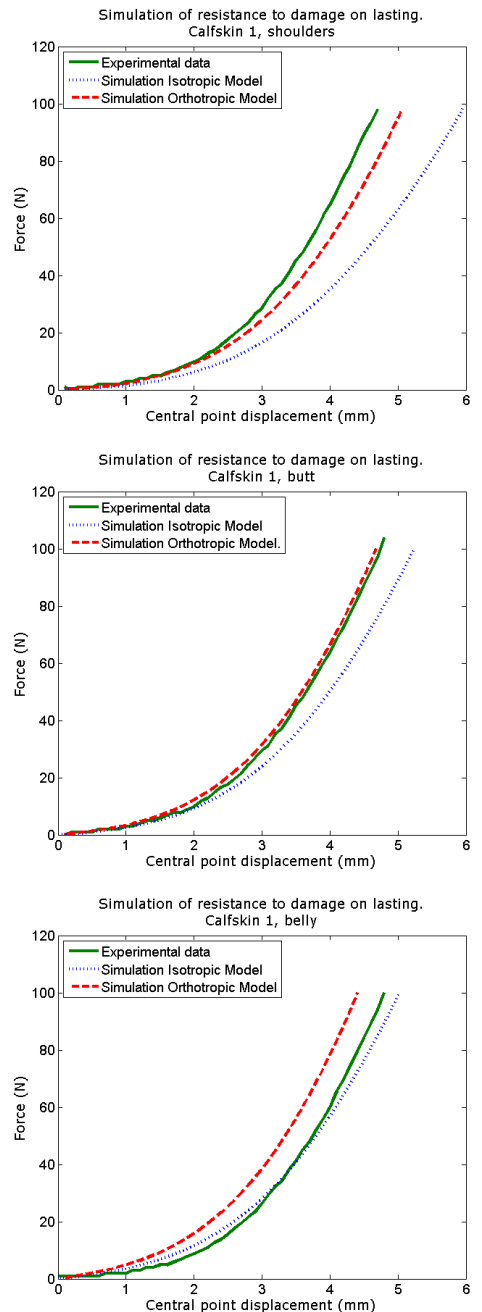

Figure 5.19: Force versus displacement of the central point of the sample in the simulation of resistance to damage on lasting for samples cut in the shoulder (top), butt (middle), and belly (bottom) areas of calfskin 1. 
Chapter 5. Behavior of the shoe upper material in gait
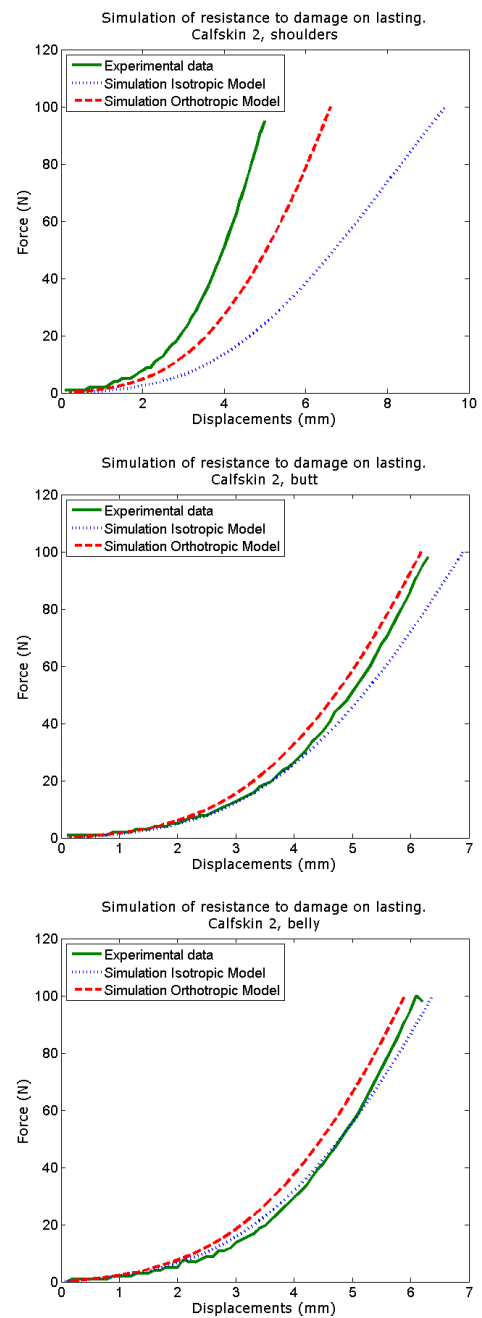

Figure 5.20: Force versus displacement of the central point of the sample in the simulation of resistance to damage on lasting for samples cut in the shoulder (top), butt (middle), and belly (bottom) areas of calfskin 2. 
the behavior for the perpendicular and parallel areas in all the cases except for the belly area of skin 2 (Fig. 5.23, bottom).
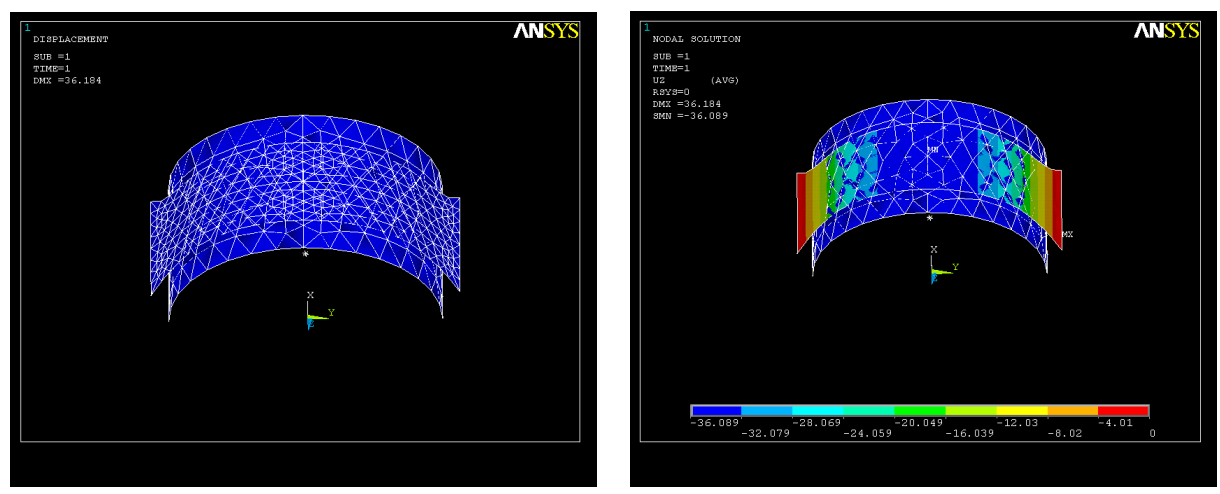

Figure 5.21: Simulation of the last forming test using ANSYS for the butt area of skin 2. Deformed mesh (left) and displacements (right).

\subsection{Discussion}

The parameters of the models proposed in this work were obtained from two kinds of tests: standard tensile tests for the extraction of the elastic moduli of the calfskins, and tests based on the experiments of Lin \& Hayhurst to extract the Poisson's ratios. To validate the proposed models for the behavior of these leathers when they are used as shoe uppers, the also standard test for the measurement of the resistance to damage on lasting was used, as well as a specific experiment designed to model the deformation of the shoe upper in a shoe forming process using lasts [111]. Although some of the assumptions are unavoidable: for example, the simplified estimation of the Poisson's ratios in the normal directions to 
Chapter 5. Behavior of the shoe upper material in gait
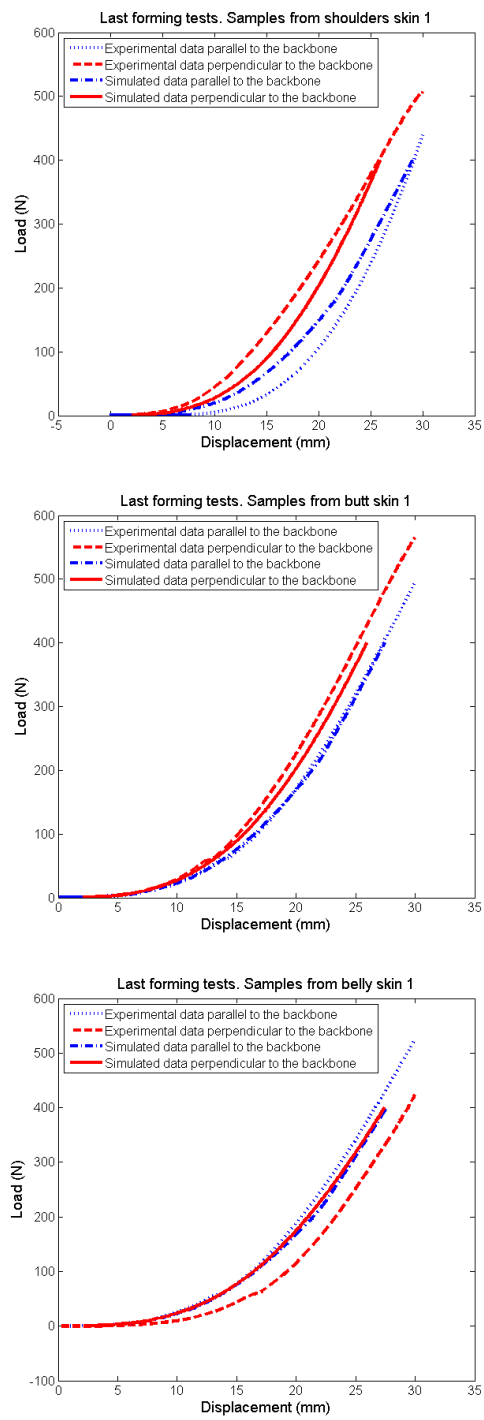

Figure 5.22: Force versus displacement of the central point of the sample in the simulation of the last forming tests for samples cut in the shoulder (top), butt (middle), and belly (bottom) areas of the calfskin 1. 

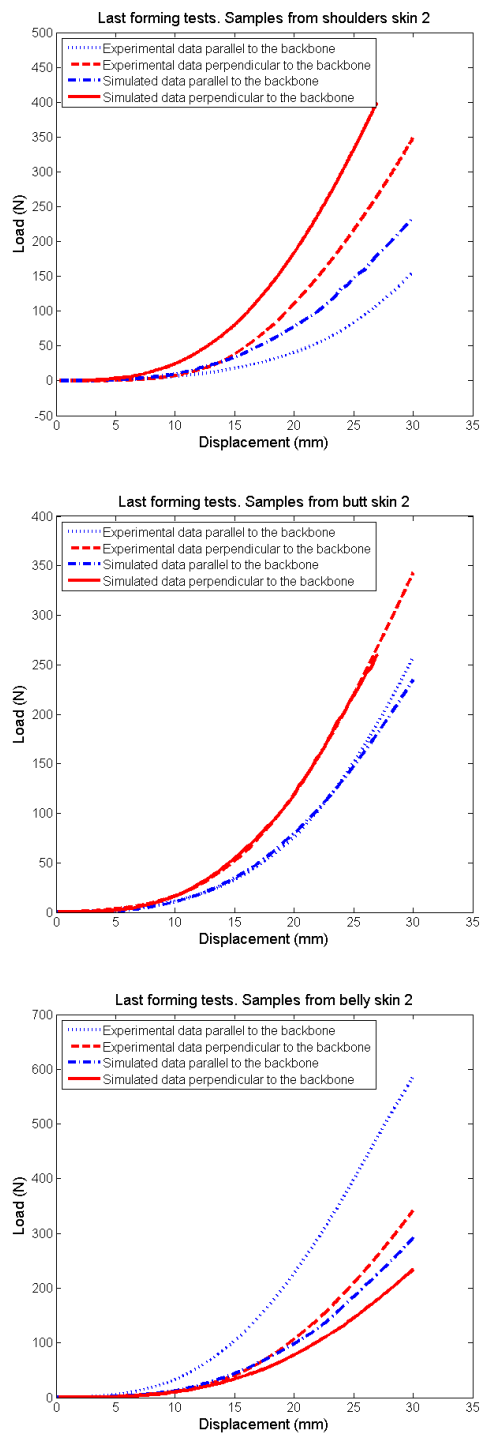

Figure 5.23: Force versus displacement of the central point of the sample in the simulation of the last forming tests for samples cut in the shoulder (top), butt (middle), and belly (bottom) areas of the calfskin 2. 
Chapter 5. Behavior of the shoe upper material in gait

the direction in which the tensile test was performed or, the estimation of the shear moduli as an average of the isotropic behavior, the mechanical behavior of the calfskin can be simulated with a linear orthotropic model when it is used as shoe upper material, which considers large deformations, and membrane and bending situations.

The main problem of the orthotropic model has been the measurement of the Poisson's ratios and shear moduli since to the author's knowledge, there does not exist any method to measure these parameters for the leathers in the standards. These parameters are very important in the development of the model since they can provide differences in the results, as it is shown in Figs. 5.19 and 5.20. For the skin areas where the Young's moduli in the two directions parallel and perpendicular to the backbone were very similar, there was a notable difference between the two models, isotropic and orthotropic, mainly due to the differences between the values of the Poisson's ratios. Therefore, tests for the measurement of these ratios as well as for the measurement of the shear moduli must be designed for the leather in order to analyze the accurate behavior of the model.

In this work, a linear approximation has been performed at the beginning of the curves below $5 \mathrm{MPa}$, due to the fact that the dorsal pressures measured on the foot surface are under this value, as the graphs of the peak pressures show in Fig. 4.7 in Chapter 4, or as it has been reported in $[2,91,92]$. Above this value, non-linearities appear and therefore, new constitutive equations should be used to model the leather behavior. Obviously, the Lin \& Hayhurst model can be used in this case. To the author's knowledge, the Lin \& Hayhurst model has not been implemented in any FE commercial software. Therefore, it should be introduced as a model defined by the user in available commercial softwares in order to be used. This can be carried out in the commercial software ABAQUS, for example, using the UMAT subroutine, which is mainly based on determining 
the Jacobian matrix, $\partial \triangle \sigma / \partial \triangle \varepsilon$ for the mechanical constitutive model. In appendix I, the UMAT subroutine for the Lin \& Hayhurst membrane behavior is provided, although to be used with shell elements also the transverse shear stiffness should be defined (see Abaqus User Subroutines Reference Manual).

\subsection{Summary}

The results of this chapter show how the behavior of the calfskin analyzed in this thesis can be modeled with an orthotropic model when it is used as shoe upper material. However, there are many other kinds of leathers that can be used as shoe upper materials and which can even present different behavior to that presented by the calfskin. In addition, the shoe uppers are not homogeneous; many other components made of different materials can be part of the shoe uppers. The behavior of these components will also have to be studied if the deformation of shoes with these components wants to be analyzed. This highlights the difficulty to establish a constitutive equation for any shoe upper whose behavior wants to be simulated in the simulator. Therefore, other techniques of modeling should also be studied. 
Chapter 5. Behavior of the shoe upper material in gait 


\section{Chapter 6}

\section{Artificial Neural Networks for modeling flexibility tests}

This chapter shows a procedure based on Artificial Neural Networks (ANNs) to reduce the number of flexibility tests that can be performed to characterize the materials used as shoe uppers. These tests provide the relationship between the exerted force by a sphere pushing a sample of material and the displacement of its central point. Using the elastic parameters of the material as inputs to an ANN, it is possible to find a neural model that provides a single equation for the relationship between force and displacement instead of a different characteristic curve for each material. The achieved results show the suitability of the novel proposed approach [71].

\subsection{Introduction}

The deformation of the shoe upper material in gait is mainly due to the contact with the foot dorsum. In order to model this contact, a whole 
Chapter 6. Artificial Neural Networks for modeling flexibility tests

study about the elasticity of the shoe upper material must be performed. Several tests must be carried out to determine the elastic parameters that characterize it. In this chapter, a model to predict the characterization of the shoe upper materials is proposed. The model is aimed at reducing the quantity of elasticity tests necessary to determine the behavior of the materials subjected to this kind of contact. In particular, the goal is modeling the exerted force on different shoe upper materials as a function of the displacement and the material parameters in a flexibility test. The flexibility tests have been designed to simulate the contact between the foot and the shoe upper. The final aim is to have a general model able to simulate these tests for different materials without having to carry them out. The advantage of this general model is that it would be possible to obtain the characterization for new materials without performing the test, if the new materials are not considerably different to those used to obtain the neural model. This way, the quantity of experimentation needed to characterize the shoe upper materials will be drastically reduced.

As the foot dorsum has little soft tissue, the foot dorsum behavior can be assumed to be determined by the behavior of the bones that form it [10], and in this sense, a set of tests called flexibility tests were designed by the IBV, Institute of Biomechanics of Valencia (http://www.ibv.org/). These tests were based on simulating how a bone pushes a piece of material. These tests were aimed at being applied to any kind of material, not necessarily leathers, that can be used as shoe upper material. These tests consist of applying a controlled force by means of a sphere that simulates a bone on a sample of material fixed to a gag. The result of these tests is the force that the sphere exerts on the sample versus the displacement of the central point of the material sample (Fig. 6.1).

Three materials that are commonly used in the footwear manufacture were subjected to these tests in order to simulate how the material was deformed 
6.1. Introduction
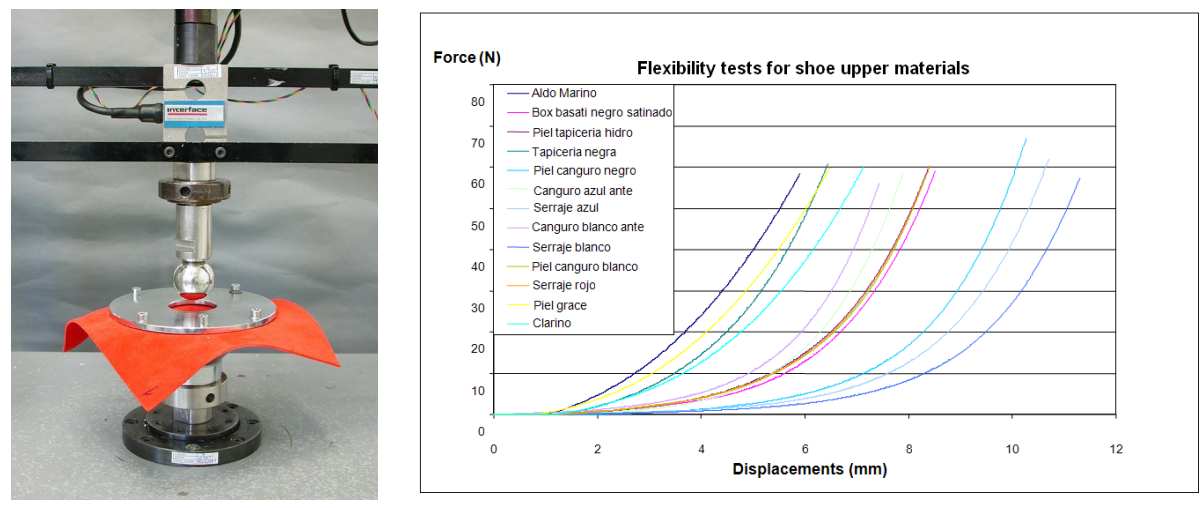

Figure 6.1: Flexibility test (left). Force versus material displacement (right). Image from $I B V$.

by the pressure of the bones. With the results of these tests, which provide the curves of the force exerted versus the displacement of the central point of the material shell, it is possible to derive an expression that characterizes the material. Different materials are usually independently modeled. However, there are several approaches within Machine Learning (ML) that allow obtaining a single model for all the materials. In this chapter, we show how a Multilayer Perceptron (MLP) is able to characterize all three materials with a single equation that has the following inputs: the elastic parameters of the corresponding material (Young's modulus and Poisson's ratio), the thickness, and the displacement of the central point of the material shell. The output of the network is given by the force that the sphere exerts for that displacement.

The relevance of obtaining a single equation for the three materials stems from the fact that this equation will model the behavior under contact of most of the materials used as shoe uppers in footwear manufacture, just 
Chapter 6. Artificial Neural Networks for modeling flexibility tests

by introducing their elastic parameters and the thickness. It should be emphasized that this procedure can be applied to model the behavior under contact of other materials like for example, foams used as reinforcements or shoe counters, and hence, the contact with the foot surface of any kind of shoe upper could be modeled. ANNs involve a novel approach with respect to the classical techniques of modeling each material separately.

It should be pointed out that ML in general, and Artificial Neural Networks (ANNs) in particular, have been widely applied to many different fields but their use in Biomechanics is still limited. In Biomechanics, ANNs have been applied only for the characterization of the human movement and for the biomechanical modeling of the movement control [5], but they had not been used to characterize any material before [71]. Here, ANNs also involve a novel approach with respect to the classical techniques of modeling.

Section 6.2 describes the Artificial Neural Networks in general and the Multilayer Perceptron in particular, as well as the learning algorithm used in this thesis: the backpropagation algorithm. Section 6.3 describes the experimental setup used for the modeling of the flexibility tests. Section 6.4 presents the obtained results, and Section 6.5 analyzes the implications of the developed tool.

\subsection{Artificial Neural Networks}

There are two main approaches to describe Artificial Neural Networks (ANNs). Some authors describe ANNs as biological models that can be applied to engineering problems [45]. However, other authors consider ANNs as mathematical models related to statistical models, either linear 


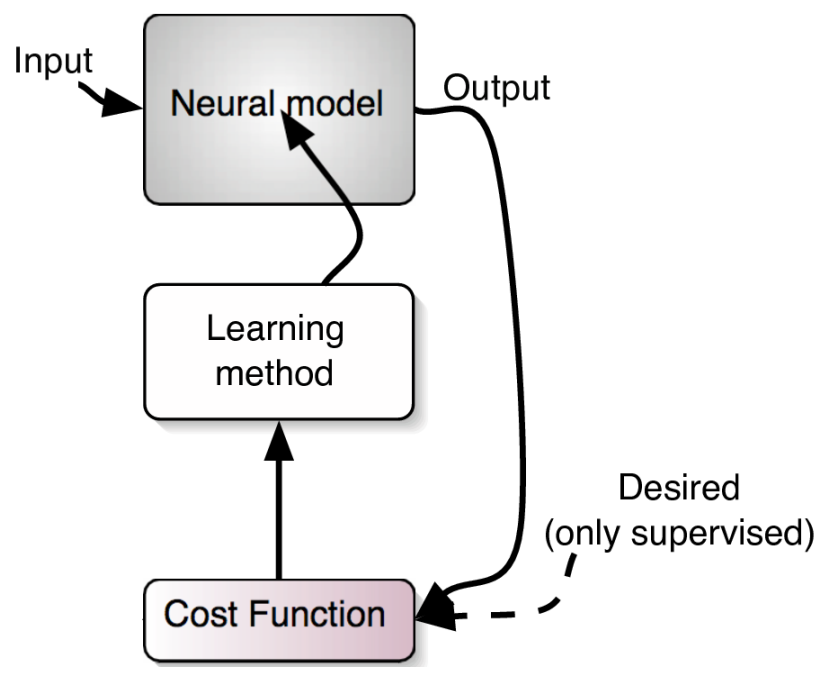

Figure 6.2: Schematic diagram of a neural model. Image from [49].

or non-linear $[46,47,48]$. Chapter 7 and Chapter 8 of this thesis are focused on the mathematical approach of the artificial neural models. In this approach, an artificial neural system can be described as it is shown in Fig. 6.2. In this figure, three main parts can be observed:

- Neural model. It is the structure used to solve a certain problem. This model can be either linear or non-linear, and it can have one or more than one outputs, it can consist of a combination (linear, nonlinear, hierarchical, etc.) of simple neural models. The performance of this model depends on several parameters, called synaptic weights, which determine the complexity of the neural model.

- Cost function. It provides an evaluation about the quality of the solution obtained by the neural model. If an external signal to carry out this evaluation is available, then the neural model is called supervised model. If an external signal is not available, then the neural 
Chapter 6. Artificial Neural Networks for modeling flexibility tests

model is called unsupervised model [113].

- Learning method. The task of the learning algorithm is to obtain those parameters of the neural models that provide a solution to the tackled problem. If this solution does not exist, the task of the learning algorithm is to find the best possible solution according to the criterion set by the cost function. Therefore, the learning algorithm depends on the chosen cost function.

Summarizing, there are three basic elements in an artificial neural model. Since all these elements can change independently to the others, there is a huge amount of models/cost functions/ learning algorithms [45]. In this thesis, we have used the most widely used neural model, the Multilayer Perceptron (MLP) trained by supervised algorithms.

\subsubsection{The Multilayer Perceptron}

In this Section, the Multilayer Perceptron (MLP) is described. It is probably the most widely used neural network due to its interesting characteristics: universal function approximator and non-linear classifier. The MLP has shown excellent results in many different applications [49].

\section{Neurons}

An MLP is a neural network made up by multiple, similar, non-linear processing units, termed neurons by analogy to the integrate-and-fire action of neural cells in biological nervous systems. Each neuron carries out a many-to-one mapping from its inputs to a single scalar output. The principal components of a neuron model are the synaptic weights, a sum 
function that relates these synaptic weights with the input vector, and the activation function $[114,115]$.

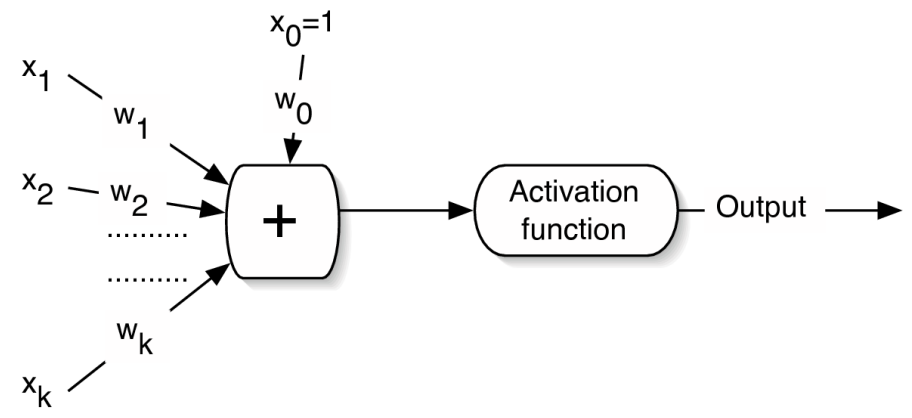

Figure 6.3: Schematic diagram of the most used neuron model.

A general schematic diagram of a neuron model is shown in Fig. 6.3. First, the sum of the product between input vectors and synaptic weights is computed. Let be $\mathbf{w}=\left[w_{0}, w_{1}, \ldots, w_{k}\right]$ the synaptic weight vector and $\mathbf{x}=\left[1, x_{1}, \ldots, x_{k}\right]$ the input vector, this sum can be given by the scalar product of both vectors. From a biological point of view, this function represents the action of the inputs produced in the axons of the biological neurons [45]. The coefficient $w_{0}$ plays a relevant role in the processing of the neuron, and it is called threshold or bias.

The activation function is very important since the neural network capabilities to solve complex problems stem from it. It is a non-linear function that provides the output of the neuron and whose argument is the aforementioned combination between synaptic weights and input vector. The most used functions are the sign function, the hyperbolic tangent, the sigmoidal function (hyperbolic tangent modified by restricting the range of values between 0 and 1), and the Gaussian function.

- Sign function. It was the first used activation function in an artificial 
neural model [45]. This function proposes a crisp separation of the input data, so that they are classified as \pm 1 . This function is defined as Eq. 6.1 shows:

$$
\varphi(x)=\left\{\begin{array}{cl}
-1 & \text { if } x<0 \\
1 & \text { if } x \geq 0
\end{array}\right.
$$

- Sigmoidal function. Since many learning algorithms need a differentiable activation function, and the sign function can not be differentiated in the origin, the sigmoidal function was proposed. This function is given by the Eq. 6.2:

$$
\varphi(x)=\frac{a}{1+e^{-b x}}
$$

where $a$ stands for the amplitude of the function and $b$ is the slope in the origin; the higher the value of $b$, the closer the sigmoidal function to the sign function (but with the outputs ranging between 0 and a). The bipolar version of the sigmoidal function (outputs ranging between $-a$ and $+a$ ) is given by the hyperbolic tangent, Eq. 6.3:

$$
\varphi(x)=a \frac{1-e^{-b x}}{1+e^{-b x}}
$$

- Gaussian function. An alternative activation function is used in a different type of neural networks, known as Radial Basis Function (RBF), Eq. 6.4:

$$
\varphi(x)=K_{1} e^{-K_{2}(x-c)^{2}}
$$


being $K_{1}, K_{2}$ and $c$, values which determine the amplitude of the Gaussian function, its width, and its center, respectively. The main characteristic of this activation function, which makes different from others, is its locality. The function tends to zero from a certain value away from $\mathrm{x}$ on; this way, only in a reduced range of input values, the output of the activation function is considerably different from zero. Gaussian functions have their center and width values typically heuristically determined from the data.

\section{Architecture}

The architecture of a neural model gives information about how neurons are arranged in the neural model. In the case of MLP, as shown in Fig. 6.4, neurons are arranged in layers: one input layer, one output layer, and one or more than one hidden layers [45]. This arrangement in layers is due to the fact that the outputs of the neurons of a certain layer are used as inputs to the neurons of next layer (network without feedback) and/or to neurons of previous layers (networks with feedback or recurrent networks).

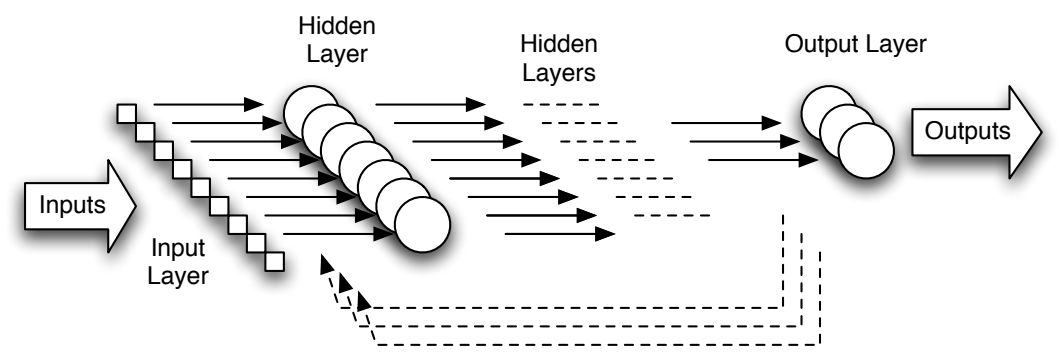

Figure 6.4: Multilayer perceptron. Dotted arrows stand for recurrent neural systems. Image from [49]. 
Chapter 6. Artificial Neural Networks for modeling flexibility tests

The number of neurons in both input and output layers is given by the problem to solve, whereas the number of hidden layers, and the neurons in each layer should be chosen by the designer of the neural network. It is shown that an MLP with one hidden layer is a universal approximator of continuous functions; if the function to model is not continuous, then two hidden layers become necessary [116]. Although there are several rules to assess the approximately optimal number of hidden neurons, there is no precise methodology to determine this number exactly [49]. Therefore, trial-and-error procedures are usually carried out to estimate the number of hidden neurons, often relying on cross-validation or other evaluation procedures.

\section{Cost function}

A neural system should be designed to present a desired behavior, hence, it is necessary to define what is desired, being used a cost function for that. The task consists of implementing the final goal of the neural system in the cost function. This is the case of supervised systems; in these systems, the model is designed to provide an output as similar as possible to a certain desired signal (Fig. 6.2). An error signal is defined from the difference between the neural model output and the desired values. The goal of the neural model is to obtain an error signal equal to zero; therefore, if an error function is defined provided that its minimum corresponds to a zero error, then the goal is transformed in a problem of function minimization $[117,118]$. The most used cost functions can be found in Table 6.1. In this table, the desired signal is denoted by $d$, the output of the neural model by $o$, and the error signal by $e$, being $e=d-o$. The parameter $p$ is any natural number. The parameter $\beta$ controls the error ranges in which a certain cost function is used; this parameter is used in those cases in which 
different subfunctions are defined for a certain cost function. In the case of the logistic cost function, $\alpha$ controls the robustness to outliers [49].

Table 6.1: Most used cost functions

\begin{tabular}{|c|c|}
\hline Name & Cost function \\
\hline $\mathbf{L}_{2}$ & $e^{2}$ \\
\hline $\mathbf{L}_{1}$ & $|e|$ \\
\hline $\mathbf{L}_{\mathbf{p}}$ & $\frac{1}{p}|e|^{p}$ \\
\hline Entropic & $\left\{\begin{array}{c}d \log \left(\frac{d}{o}\right)+(1-d) \log \left(\frac{1-d}{1-o}\right) \\
0 \leq o \leq 1\end{array}\right.$ \\
\hline Logistic & $\frac{1}{\alpha} \log (\cosh (\alpha e))$ \\
\hline Huber & $\left\{\begin{array}{cl}0.5 e^{2} & \text { for }|e| \leq \beta \\
\beta|e|-0.5 \beta^{2} & \text { otherwise }\end{array}\right.$ \\
\hline Talvar & $\begin{cases}0.5 e^{2} & \text { for }|e| \leq \beta \\
0.5 \beta^{2} & \text { otherwise }\end{cases}$ \\
\hline
\end{tabular}

\subsubsection{Backpropagation algorithm}

A learning algorithm is a procedure which adapts the parameters and/or architecture of the neural model in order to solve a certain problem. The algorithms to adapt the architecture of the MLP are known as pruning methods or growing methods, depending on the strategy to carry out this adaptation [113]. Pruning methods have been more widely used than grow- 
Chapter 6. Artificial Neural Networks for modeling flexibility tests

ing methods. Learning algorithms to adapt the parameters of the neural model tend to be based on the minimization of the cost function chosen to solve the posed problem. First neural models were formed by only one neuron and they used $L_{2}$ as cost function, so that the minimum was obtained by solving a system of linear equations [114]. However, the practical application of these models involved solving this system of equations in every instant of processing; taking into account the huge number of unknown quantities and the technological state of those years (1950-1960), other approaches were researched in order to solve these systems of equations. The most important characteristics that a learning algorithm must show, are the following [113]:

- Efficiency. Ability to solve the problem with the minimum computational burden.

- Robustness. The algorithm should present immunity to undesired noise.

- Independence on the initial conditions. The algorithm should show similar solutions independently of the values used to initialize the algorithm.

- Generalization capabilities. The algorithm should provide the adequate outputs when inputs different to the training data set, are used.

- Scalability with the size and complexity of the data. The algorithm should have a computational burden that does not strongly depend on the dimensionality and size of the problem to solve.

The backpropagation algorithm (BP) was the first algorithm used for MLP adaptation [119, 120,121], and it is still the most known and used nowa- 
days. The goal pursued by the algorithm is to obtain the minimum of the cost function, which is usually denoted as $J$. The actual solution of $J$ is computationally unfeasible in many applications. Moreover, the solution of $J$ in a certain instant may be uninteresting in many cases since the properties of the input signals to the neural model may be time-dependent and the neural models must adapt to these changes. Then, an iterative solution is proposed by means of Eq. 6.5, where $\mathbf{w}$ represents the synaptic weights of the neural model and the subscript stands for the time instant.

$$
\mathbf{w}_{n+1}=\mathbf{w}_{n}+\triangle \mathbf{w}_{n}
$$

Eq. 6.5 is used to find out the optimal increase/decrease in the synaptic weights that enables to find the minimum of the function. A low computational burden approach is based on a geometrical analysis of the problem. This analysis is based on finding the direction of the minimum from a certain point $\mathbf{w}_{n}$ in the synaptic weight space of the neural model. Since the function gradient points to the direction of the function maximum, the approach will be based on finding the function minimum by moving the synaptic weights in the opposite gradient direction. Thus, $\triangle \mathbf{w}_{n}=-\alpha \nabla_{\mathbf{w}_{n}} J$, which is known as delta rule, where $\alpha$ is the so-called learning rate or adaptation constant $[46,117,122]$. Therefore, Eq. 6.5 provides Eq. 6.6.

$$
\mathbf{w}_{n+1}=\mathbf{w}_{n}-\alpha \nabla_{\mathbf{w}_{n}} J
$$


Chapter 6. Artificial Neural Networks for modeling flexibility tests

The BP algorithm based on Eq. 6.6 is a gradient-descent algorithm which backpropagates the error signals from the output layer to the input layer, thus optimizing the values of the synaptic weights through an iterative process. Therefore, two stages can be considered:

- Feed-forward propagation: The output of the neural network is obtained, and then, the error is computed by comparing this output with the desired signal.

- Backpropagation: Depending on the error between the network output and the desired values, the algorithm optimizes the values of the synaptic weights by means of error backpropagation from the output layer to the input layer, and through the hidden layers.

To describe the algorithm, an MLP with an input layer, a hidden layer and an output layer is considered. The extension to more hidden layers is trivial.

Given the inputs to the network $\left\{x_{1}, \ldots, x_{n}\right\}$, which are the inputs to the neurons of the hidden layer, $x_{0}= \pm 1$ the input for the bias $w_{m 0}$, and $\left\{w_{m 1}, \ldots, w_{m n}\right\}$ the synaptic weights that connect the inputs to the hidden neuron $m$, the linear combination provided by the sum function of the $m$ neuron in the $t$ iteration is given by Eq. 6.7.

$$
v_{m}(t)=\sum_{i=0}^{n} w_{m i}(t) x_{i}
$$

If $\varphi$ stands for the activation function, the output of the $m$ neuron is given by Eq. 6.8 . 


$$
y_{m}(t)=\varphi_{m}\left(v_{m}(t)\right)
$$

The $\left\{y_{m}\right\}$ set defines the outputs of the hidden layer neurons. Thus, they will be the inputs to the output layer. If $\left\{h_{p 1}, \ldots, h_{p r}\right\}$ stand for the synaptic weights that connect the inputs $\left\{y_{m}\right\}_{m=1}^{r}$ to the output $p$ neuron, being the bias $h_{p 0}$, and $\mathrm{r}$ the number of hidden neurons, the linear combination provided by the sum function of the $p$ neuron in the $t$ iteration will be as shown in Eq. 6.9.

$$
z_{p}(t)=\sum_{j=0}^{r} h_{p j}(t) y_{j}(t)
$$

If $\phi$ is the activation function of the output layer neurons, the output of the neuron $p$ will be as Eq. 6.10 shows.

$$
o_{p}(t)=\phi_{p}\left(z_{p}(t)\right)
$$

If $d_{p}$ stands for the desired output, the error is defined as shown in Eq. 6.11.

$$
e_{p}=d_{p}-o_{p}
$$


Chapter 6. Artificial Neural Networks for modeling flexibility tests

The iterative process that updates the synaptic weights until reaching their optimum values is given by the delta rule, and it is expressed as shown in Eq. 6.12, where $w$ is the synaptic weight to be updated and $\alpha$ the adaptation constant (or learning rate) that measures the learning speed of the considered weight.

$$
\triangle w(t)=-\alpha \frac{\partial J}{\partial w(t)}
$$

In the learning process of the backpropagation algorithm, the first updated weights are the weights of the output layer. Then, the partial derivative of the cost function with respect to these synaptic weights can be obtained applying the chain rule as shown in Eq. 6.13.

$$
\frac{\partial J}{\partial h_{p j}(t)}=\frac{\partial J}{\partial e_{p}(t)} \frac{\partial e_{p}(t)}{\partial o_{p}(t)} \frac{\partial o_{p}(t)}{\partial z_{p}(t)} \frac{\partial z_{p}(t)}{\partial h_{p j}(t)}
$$

The results of the first partial derivative depends of the cost function $J$. If $L_{2}$ is chosen, which is the most habitual, the result will be $2 e_{p}(t)$. The result of the second partial derivative is easily obtained from Eq. 6.11 and it is equal to -1 . The third derivative depends of the activation function, and it can be obtained from Eq. 6.10 as Eq. 6.14 shows.

$$
\frac{\partial o_{p}(t)}{\partial z_{p}(t)}=\phi^{\prime}\left(z_{p}(t)\right)
$$


Finally, the result of the forth derivative is obtained from Eq. 6.9 as Eq. 6.15 shows.

$$
\frac{\partial z_{p}(t)}{\partial h_{p j}(t)}=y_{j}(t)
$$

Therefore, the update of the output layer weights is given by Eq. 6.16.

$$
\triangle h_{p j}(t)=2 \cdot \alpha \cdot e_{p}(t) \cdot \phi^{\prime}\left(z_{p}(t)\right) \cdot y_{j}(t)
$$

The next step is to update the weights of the hidden layer neurons. The partial derivative of the cost function with respect to these weights is also obtained with the chain rule and it is expressed as Eq. 6.17 shows.

$$
\frac{\partial J}{\partial w_{m i}(t)}=\sum_{p} \frac{\partial J}{\partial e_{p}(t)} \frac{\partial e_{p}(t)}{\partial o_{p}(t)} \frac{\partial o_{p}(t)}{\partial z_{p}(t)} \frac{\partial z_{p}(t)}{\partial y_{m}(t)} \frac{\partial y_{m}(t)}{\partial v_{m}(t)} \frac{\partial v_{m}(t)}{\partial w_{m i}(t)}
$$

Therefore, the update of the weights is obtained from the partial derivative results as shown in Eq. 6.18.

$$
\triangle w_{m i}(t)=2 \cdot \alpha \cdot \sum_{p} e_{p}(t) \cdot \phi^{\prime}\left(z_{p}(t)\right) \cdot h_{p m}(t) \cdot \varphi_{m}^{\prime}\left(v_{m}(t)\right) \cdot x_{i}
$$


Chapter 6. Artificial Neural Networks for modeling flexibility tests

In the case of more than one hidden layer, the process is the same. The algorithm is backpropagated from the output layer through all the hidden layers up to the input layer. Thus, each hidden layer adds its corresponding factor to the update algorithm.

\section{Drawbacks of the backpropagation algorithm}

In spite of the advantages offered by the $\mathrm{BP}$ algorithm, it also shows a number of drawbacks that should be known [45, 113, 123]:

- Neuron saturation. Since the derivative of the activation function appears in the weight update, and this derivative equals zero for the most used activation functions in the function extremes, weights are not updated in these zones although the modeling error may be different from zero. Therefore, the neural network will be unable of modifying the synaptic weights independently of the committed error.

There are some methods to solve this problem. One possibility is to use activation functions whose derivatives present a value different from zero. Another possibility is to modify the activation function as: $\varphi_{\beta}(x)=\beta x+(1-\beta) \varphi(x)$, where $1 \geq \beta \geq 0$. In this case, $\beta=1$ initially; thus, the derivative of the activation function is equal to 1 . Later, $\beta$ will decrease while the network is learning up to achieve $\beta=0$; what corresponds to the situation when the modified activation function is equal to the original. This method avoids the neuron saturation at the beginning to the learning process that it is the moment when this may occur with more probability.

Other possibility is that the amplitude and the slope of the sigmoidal function (if this is the function chosen as activation function) change 
in an adaptative way while the network learns. The sigmoidal function was given by Eq. 6.2:

$$
\varphi(x)=\frac{a}{1+e^{-b x}}
$$

Therefore, the update of the $a$ and $b$ parameters can be formulated using a decreasing of the cost function gradient as Eq. 6.19 shows.

$$
\begin{aligned}
& a(t+1)=a(t)-\alpha \nabla_{a} J \\
& b(t+1)=b(t)-\alpha \nabla_{b} J
\end{aligned}
$$

Another analogous possibility can be formulated in the case of the hyperbolic tangent is used as activation function.

- Weight initialization. Weight initialization is basic in order to achieve a good modeling. Since the learning algorithm is based on finding the minimum of the error function that is closest to a certain initial point, this minimum may be a local minimum, not a global one, and therefore this initial point is fundamental for ensuring that the network finally achieves a global minimum. There are some algorithm to avoid the local minimum problem, one of them is the ERA (Expanded Range Approximation) algorithm. This algorithm modifies the backpropagation algorithm compressing the desired outputs towards their mean value at the beginning of the learning and extending them up to their original value at the end of the training process. Thus, a global minimum is reached [124]. 
Chapter 6. Artificial Neural Networks for modeling flexibility tests

Weight initialization also affects algorithm behavior in the convergence speed; the convergence speed depends not only on the learning rate, but also on the initial distance to the minimum. It affects the neuron saturation too since large values of the weights can also involve a neuron saturation.

- Plateaus. Weight update is proportional to the derivative of the error function. This derivative equals zero or a very low value on the flat parts of the error function. Therefore, weights are hardly updated.

- Choice of the learning rate. Too high values may involve instabilities while too low values may make the converge speed very slow. There are many algorithms which propose strategies to find an optimal learning rate. They tend to be based on the following claim: "the value of the learning rate should be high far away from the minimum and should be low near the minimum". For example, one of the classical approaches is adding the momentum term to the delta rule defined to update the synaptic weights. This term is given by the increments of the previous weights as shown in Eq. 6.20.

$$
\triangle w(t)=-\alpha \cdot \triangle J+\mu \cdot \triangle w(t-1)
$$

The new term controls the approaching speed to the minimum, and the momentum coefficient $\mu$ controls the importance of this correction term.

- Early stopping. There are different criteria to carry out the learning stopping, such as, to fix a number of epochs (the computation of all the outputs of the neural model for all the available input patterns) in 
6.3. Materials and methods

advance, an error threshold, to find plateaus in the cost function, etc. Data are usually split into two sets: a training set and a validation set. The former is used to train the network, whereas the latter is used to check the behavior of the network with patterns different from those used in the training process. The goal is to obtain a model with good generalization properties. The generalization error tends to decrease as the learning process is progressing until a certain epoch, in which the generalization error starts to increase because the network is overfitting the patterns of the training data set. When this change in the tendency of the generalization error is observed, the learning must be stopped. This procedure is known as crossvalidation. There are also different criteria to decide the rate of patterns that should be assigned to each data set: $66 \%$ of the patterns to the training set, a small percentage if there is a large amount of data available, and there are also dynamic processes to carry out the selection of these sets $[46,113]$.

- Architecture choice. The number of hidden neurons and layers is a difficult choice, being determined in many cases by using trialand-error procedures. Nonetheless, there also pruning and growing methods that are used to find optimal structures [125, 126].

Some other modifications to the backpropagation algorithm can be found in $[49]$.

\subsection{Materials and methods}

Samples of three of the upper materials used in the manufacture of the shoes used to measure the dorsal pressures in Chapter 5 were subjected 
Chapter 6. Artificial Neural Networks for modeling flexibility tests

to these tests. In particular, samples of the materials MU05-0531, MU050532, MU05-0533 were provided by the footwear manufacturer to perform the tests (Table 6.2, extracted from Table 4.1).

Table 6.2: Data of the materials used in the flexibility tests.

\begin{tabular}{lccc}
\hline Material & $\begin{array}{c}\text { Thickness } \\
\text { mm }\end{array}$ & $\begin{array}{c}\text { Young's modulus } \\
\text { MPa }\end{array}$ & Poisson's ratio \\
\hline MU05-0531 & 0.75 & 28.997 & 0.042 \\
MU05-0532 & 0.64 & 9.297 & 0.047 \\
MU05-0533 & 0.77 & 8.616 & 0.064 \\
\hline
\end{tabular}

To evaluate the upper elasticity, the IBV (Institute of Biomechanics of Valencia) carried out tests that enabled to determine the stiffness of the upper material simulating the pressure exerted by a bone. The tests consisted of applying a controlled force by means of a metallic sphere of diameter 3.6 $\mathrm{cm}$ on a sample of material fixed to a gag (Fig. 6.1 (left)).

Samples of the three upper materials were subjected to these tests. A gag of $40 \mathrm{~mm}$ of interior diameter was used. The tests were quasi-static, the velocity was $0.08 \mathrm{~mm} / \mathrm{s}$ and the maximum force applied was $60 \mathrm{~N}$. The results are shown in Fig. 6.5. These graphs represent the force that the sphere exerted versus the displacement of the central point of the shell of material.

The use of ANNs is proposed to model these flexibility tests. The aim is to obtain a general model for the simulation of these tests that allows to characterize the force versus displacement for different materials at the same time. This way, it would no longer be necessary the individual characterization of each material, thus reducing the number of tests to carry out. An additional advantage is that with a general model, it would also be possible to obtain the characterization for new materials using the same 

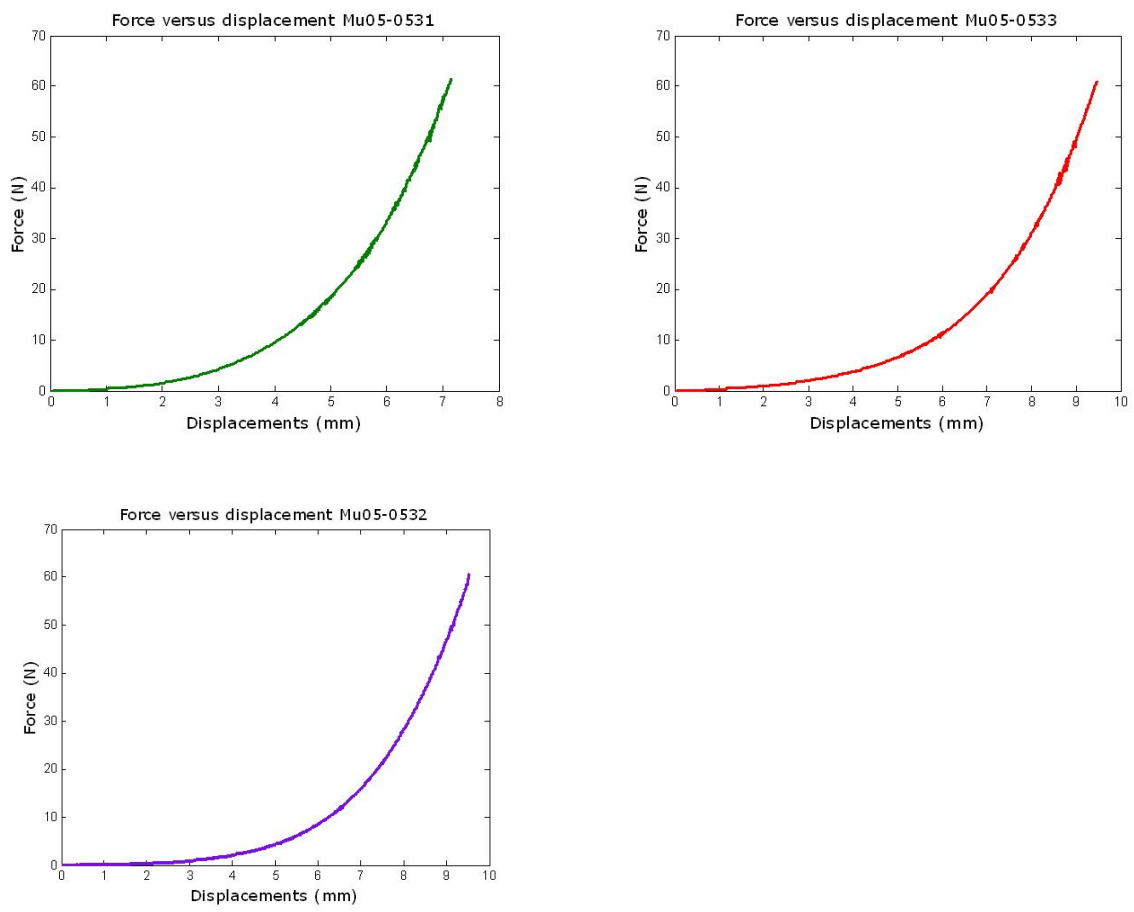

Figure 6.5: Force versus displacement for the three material under study. 
Chapter 6. Artificial Neural Networks for modeling flexibility tests

model, albeit results should be understood with caution if the new material considered is considerably different to those used to obtain the neural model.

A Multilayer Perceptron (MLP) was used for modeling these tests since it is a universal function approximator with generalization capabilities which are especially interesting in real problems such as that tackled in this work $[117,113,127]$. An MLP is composed of a layered arrangement of artificial neurons in which each neuron of a given layer feeds all the neurons of the next layer (Section 6.2.1).

The inputs $x_{i}$ to the neurons (Fig. 6.3) are multiplied by the synaptic weights $w_{i}$, which represent the synaptic connectivity between neurons. The output of a neuron is usually taken to be a sigmoid-shaped (sigmoid or hyperbolic tangent function) $\varphi$.

$$
\text { output }_{j}=\varphi\left(\sum_{i=0}^{P} w_{i j} \cdot x_{i}\right)
$$

In Eq. 6.21, the subindex $j$ represents the $j_{t h}$ neuron, $P$ the number of input patterns and $x_{0}=1$ is the bias input to the neuron. The structure is completely defined by taking $x_{i}$ to be the external inputs, and output ${ }_{j}$ to be the output of the neuron. The power of the method is demonstrated by the fact that the logistic regression is actually equivalent to one neuron with a sigmoid activation function [127]. The MLP extends this concept by using a complex architecture of neurons, as illustrated in Fig. 6.4.

For this problem, the hyperbolic tangent in hidden neurons was used for the activation function, as well as a linear function for the output neuron since 
6.3. Materials and methods

we are dealing with a modeling problem (non-linear functions in output neurons are usually employed when facing a classification problem).

The network was on-line trained using the classical backpropagation algorithm modified by the Expanded Range Algorithm (ERA) with a momentum term to speed up convergence (Eq. 6.20).

As previously mentioned, ERA is a method to mitigate the effect of local minima in gradient-descent algorithms. In this technique, the training set for the network is expanded progressively from an initial average setting to the original complete training set. The expansion process generates different sets of training data and is controlled by an homotopy parameter $\lambda \in[0,1]$. Initially, $\lambda=0$ and $d_{p}(0)=<d>$, where $\langle d>$ denotes the arithmetic mean of the original $p$ example training patterns (desired signal) and $d_{p}(0)$ is the initial setting of the desired signal for the $p_{t h}$ pattern in the ERA algorithm. This training set is then expanded for each training epoch, in a step-wise manner, until $\lambda=1$, at which point the training set is identical to the original set of input/output pairs. If it is assumed a structure with a single output neuron, as it is the case of the presented application, the expansion of the training set as a function of $\lambda, d_{p}(\lambda)$ is given by (Eq. 6.22):

$$
d_{p}(\lambda)=<d>+\lambda\left[d_{p}-<d>\right]
$$

where $d_{p}$ is the $p_{t h}$ member of the original training set.

The ability of the ERA algorithm to avoid local minima of the error function is based on three assumptions:

1. The problem defined by $\lambda=0$ has only one global minimum. 
Chapter 6. Artificial Neural Networks for modeling flexibility tests

2. The first small step $(\lambda=\eta<1)$ away from the $\lambda=0$ solution keeps the system in a global minimum.

3. The range can be progressively expanded to $\lambda=1$ without displacing the system from the global minimum at any step

These three points are satisfied for the XOR problem [124]; however, ERA can break down in more complex situations. The reason for this is that no general strategy has been found for step size variation during the course of the expansion of the training set so as to ensure global convergence. This is a difficult problem due to the dependence on the training set, the network architecture and the initialization of the synaptic weights. In this work, $\lambda$ was increased from 0 to 1 in a homogenous step-wise manner. In particular, ten steps were considered, and thus, if $t$ is the number of training iterations, $\lambda$ value will increase 0.1 each $t / 10$ iterations.

\subsection{Results}

The following features were used as inputs to the MLP model: displacement of the sphere $(\mathrm{mm})$, thickness $(\mathrm{mm})$, Young's modulus $\left(\mathrm{N} / \mathrm{m}^{2}\right)$ and Poisson's ratio. The output of the model is the exerted force by the sphere on the material sample. Hence, the MLP is aimed at modeling the exerted force depending on the displacement and the analyzed material parameters.

Experimental data was split into two data sets: a training data set to obtain the neural network model and a test data set to evaluate the model performance with different data from those used to obtain the model. This way, generalization capabilities are guaranteed. The training data set was 
formed by 6543 patterns whereas the test data set consisted of 3272 patterns. Training was early stopped by cross-validation $[117,127]$.

The model architecture (number of hidden neurons) and parameters (learning rate, momentum coefficient, and standard deviation of the random distribution of initial weights) were optimized by trial-and-error procedures. The best neural model showed an architecture $4 \times 6 \times 1$. Only a hidden layer was enough to obtain accurate results. The number of hidden layers was varied from 2 to 12 . Synaptic weights were initialized following a pseudorandom Normal distribution with mean zero and standard deviation unity. The learning rate was set to 0.01 and the momentum coefficient was set to 0.5. The optimum instant to stop the training procedure was after 2977 iterations (Fig. 6.6).

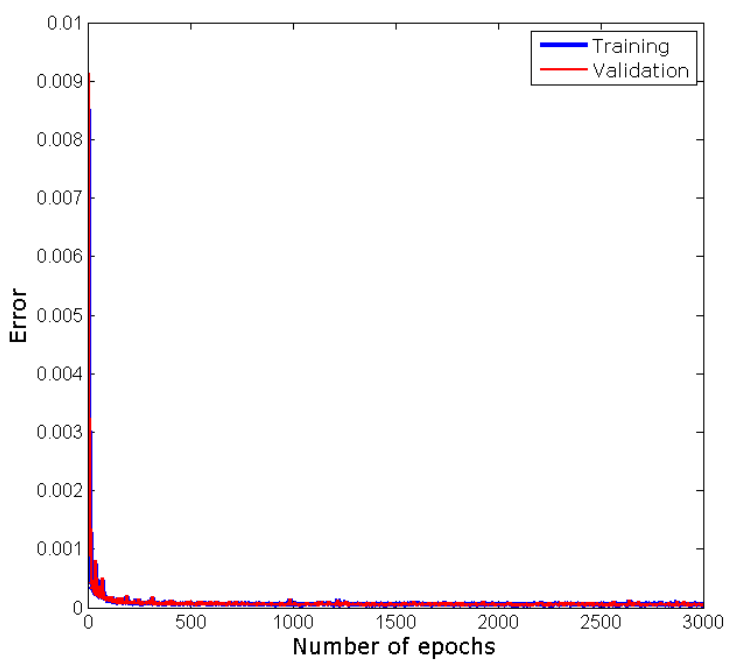

Figure 6.6: Error decay with the number of epoch for the validation and training processes.

Table 6.3 shows a summary of the achieved results. The following error 
functions are given:

- Mean Error (ME). The Mean Error is used as a measure of bias. It is defined in Eq. 6.23, where $N$ is the number of patterns used to calculate $\mathrm{ME}, d_{i}$ is the actual (desired) value of the force that should be modeled, and $o_{i}$ is the value that is predicted by the neural network. Positive values mean that the predictor tends to yield forces that are lower than the actual ones, while negative values represent an over-biased predictor. Therefore, the desired values for ME should be as close to zero as possible.

$$
\mathrm{ME}=\frac{1}{N} \sum_{i=1}^{N}\left(d_{i}-o_{i}\right)
$$

- Mean Absolute Error (MAE). The Mean Absolute Error, which is defined in Eq. 6.24, is used as a measure of accuracy. Since absolute values are used, negative errors in the prediction do not compensate positive errors, and, hence, MAE gives a fair idea of the accuracy of the predictor.

$$
\mathrm{MAE}=\frac{1}{N} \sum_{i=1}^{N}\left|d_{i}-o_{i}\right|
$$

- Root Mean Square Error (RMSE). Oftentimes, the use of RMSE is preferred to MAE as an accuracy measure. RMSE is defined as shown 
in Eq. 6.25.

$$
\mathrm{RMSE}=\sqrt{\frac{1}{N} \sum_{i=1}^{N}\left(d_{i}-o_{i}\right)^{2}}
$$

Table 6.3: Results achieved in the MLP modeling of the exerted force to the sphere. All values are expressed in Newtons.

\begin{tabular}{lcc}
\hline Error & Training data set & Test data set \\
\hline ME & 0.001 & 0.002 \\
MAE & 0.055 & 0.054 \\
RMSE & 0.110 & 0.107 \\
\hline
\end{tabular}

In order to assess the errors provided in Table 6.3, it is necessary to know the range of desired values. In particular, in our database force values were ranged from 0 to $61.71 \mathrm{~N}$, being the mean value equal to $12.86 \mathrm{~N}$, and the standard deviation $16.02 \mathrm{~N}$. Therefore, on average, relative errors are lower than $1 \%$ of the standard deviation, which is an accurate regression, as it is shown in Fig. 6.7, where part of the patterns belonging to the validation data set together with the real values are plotted. Plotted patterns have been randomly selected to provide a useful and understandable representation, since the performance for all the patterns was approximately similar.

\subsection{Discussion}

In this work, we have presented an application of ANNs to Footwear Biomechanics. In particular, the goal was modeling the exerted force on different 
Chapter 6. Artificial Neural Networks for modeling flexibility tests

Neural network modeling

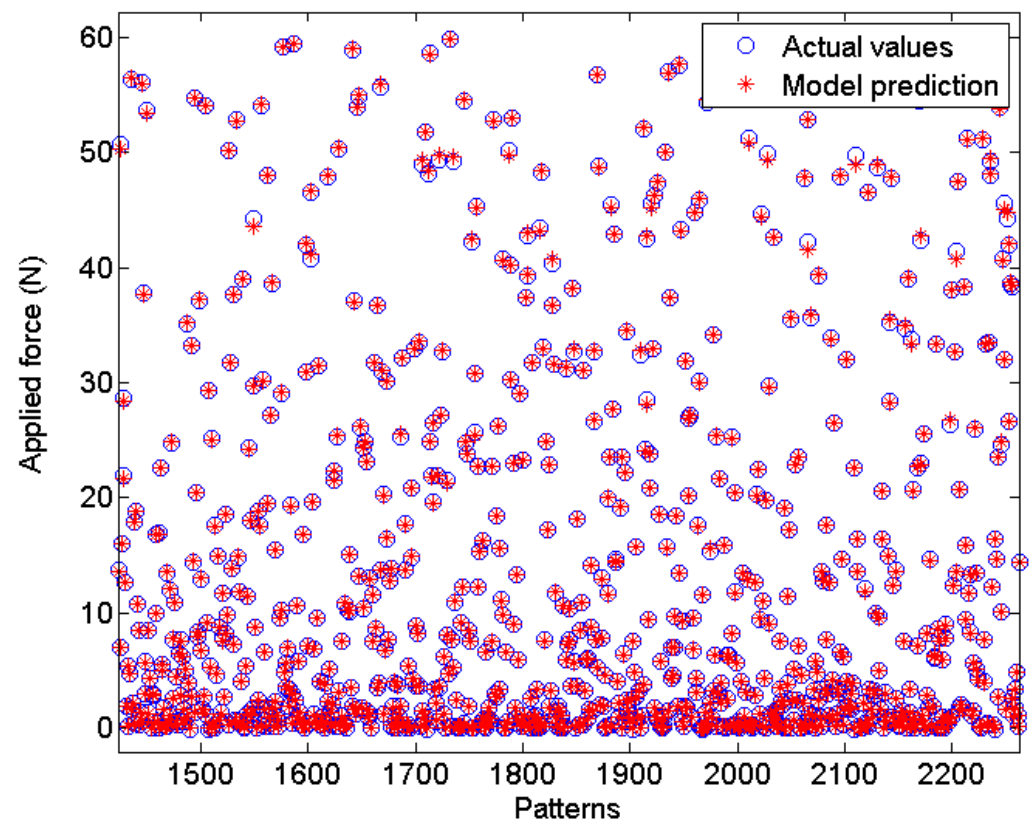

Figure 6.7: Neural network modeling (circles) and real values of force (stars) for approximately third of the patterns belonging to the validation data set (random selection of patterns) 
shoe upper materials as a function of the displacement and the material parameters in a flexibility test. The final aim was to have a general model able to simulate the flexibility tests for different materials without having to carry them out. The advantage of the obtained general model is that it would be possible to characterize new materials without performing the test, if the new materials are similar to those used to obtain the neural model (as, for example, the shoe upper materials shown in Fig. 6.1, right). This way, the quantity of experimentation necessary to characterize the shoe upper materials will be drastically reduced. Achieved results have shown that an MLP-based modeling has a high level of accuracy, and with only one model, it has been possible to model three different material behaviors. Therefore, it is possible to use a single mathematical function that given the material parameters and the corresponding displacement, models the exerted force with high accuracy.

\subsection{Summary}

The research presented in this thesis has been focused on simulating the contact between the foot and the shoe upper in a virtual way, so that a fast characterization of the used materials will be very useful to reduce the experimental costs. The aim of the tool developed in this chapter is precisely that, to reduce the tests needed to characterize a material modeling its behavior. Future work will be devoted to get the whole simulation of the shoe deformation in gait including all footwear components, so that the application of this tool to characterize components like reinforcements, insoles etc., will improve the development of the footwear virtual simulator. 
Chapter 6. Artificial Neural Networks for modeling flexibility tests 


\section{Chapter 7}

\section{Artificial Neural Networks for predicting dorsal pressures over the foot surface}

In this chapter, Artificial Neural Networks (ANNs) are proposed to predict the dorsal pressure over the foot surface exerted by the shoe upper materials while walking. As in the previous chapter, a model that is based on the Multilayer Perceptron (MLP) is used since it can provide a single equation to model the exerted pressure for all the materials used as shoe uppers. Five different models are produced, one model for each one of the four subjects (individuals) under study and an overall model for the four subjects. The goal is to find models with good generalization capabilities (i.e., models that work appropriately not only for the cases used to train the model but also for new cases) in order to have a useful predictor in routine practice. New cases may involve either new materials for the same subject or even new subjects and new materials. To accomplish this goal, 
Chapter 7. Artificial Neural Networks for predicting dorsal pressures over the foot surface

two thirds of the patterns are used to obtain the model (training data set) and the remaining third is kept for validation purposes. The achieved accuracy was very satisfactory since correlation coefficients between the predicted output and the actual pressure in the validation data set were higher than 0.95 for those models developed for individual subjects. For the much more challenging problem of an overall prediction for all the subjects, the correlation coefficient was close to 0.9 in the validation data set (i.e., with data not previously seen by the model).

\subsection{Introduction}

Given the good capabilities shown by the ANNs in the previous chapter to model the flexibility tests, the use of the ANNs was also proposed to predict the dorsal pressures on the foot surface exerted by the shoe upper materials in gait.

This approach was based on the results of the dorsal pressure distributions obtained in Chapter 4 to study the viability of constructing a generic animation of the foot to be used in the simulator. Those results showed up the similarity of the dorsal pressure distribution exerted by different shoe upper materials measured by the same sensor placed on an anatomical point of a given subject. This suggested that ANNs could be good predictors for this kind of pressures. The results of this work show that ANNs are indeed a good predictor for this kind of pressures as already published in [73]. To the author's knowledge, ANNs had never been used to model the foot-shoe contact before the work presented by the author in [71], thus being the current work another novel approach in this field.

Section 7.2 describe the data collection and methods used. Section 7.3 
7.2. Materials and methods

presents the results achieved by the predictor. Section 7.4 presents some conclusions and proposals for further work.

\subsection{Materials and methods}

Six variables were used to perform the prediction. The inputs to the neural model included the material properties, i.e., Young's modulus and Poisson's ratio, and the thickness (Table 4.1, Chapter 4), as well as the coordinates $\mathrm{x}, \mathrm{y}$, and $\mathrm{z}$ of the positions of the fourteen anatomical points on which the sensors were placed during a whole step (Section 4.2.2). The output of the model corresponded with the pressures recorded by the sensors during the step (Section 4.3).

The outcome of the neural predictor model represents an overall expression to obtain the exerted pressure for all the materials by including the material properties, the thickness, and the position of the sensor as inputs to the neural model (Eq. 7.1).

$$
\text { pressure }=\phi^{\star}\left[\sum_{i=0}^{N} h_{i} \cdot \phi\left(\sum_{j=0}^{M} w_{i j} \cdot x_{j}\right)\right]
$$

In Eq. 7.1, $\left\{x_{j}\right\}$ are the inputs of the predictor, $M$ is the number of inputs of the predictor, $N$ is the number of hidden neurons, $\left\{w_{i j}\right\}$ are the synaptic weights that connect the input layer to the hidden layer, $\left\{h_{i}\right\}$ are the synaptic weights that connect the hidden layer to the output layer, $\phi$ is the activation function of the neurons belonging to the hidden layer, and $\phi^{\star}$ is the activation function of the output neuron. 
Chapter 7. Artificial Neural Networks for predicting dorsal pressures over the foot surface

Thus, when a new material is used for a subject, it is not necessary to carry out new tests to measure the dorsal pressure curve since the MLP can extrapolate it, which drastically reduces the time and cost. This approach requires obtaining a model for each subject, although we have also produced a "generic" overall model for all the subjects. This "generic" model should provide predictions for all the materials and all the subjects. Table 7.1 shows the number of records used in each of the four subjectbased data sets as well as in the overall data set. The number of records shows the number of time samples used to measure the exerted pressure and the positions of the sensors during a complete step. Therefore, each time sample was used as a pattern to either train or test the neural models.

Table 7.1: Number of records (time samples) for the different data sets.

\begin{tabular}{lc}
\hline Data set & Number of time samples \\
\hline Subject \#1 & 125 \\
Subject \#2 & 95 \\
Subject \#3 & 95 \\
Subject \#4 & 114 \\
All subjects & 429 \\
\hline
\end{tabular}

In order to train the models, two subsets were produced for each data set. A training data set made up of two thirds of the records was used to train the model in order to provide accurate predictions. However, since the final goal was not the accurate prediction for the cases already known but rather for new ones, a validation data set made up for the remaining third of the records was used to guarantee the generalization capabilities of the models [127]. If all the available information is used to develop a model, the model tends to work very accurately for the training data but its performance may be very poor with new unseen data, i.e., the model is over-fitted to the data. In order to avoid over-fitting, the data is split 
7.3. Results of the neural prediction

into a training data set used to develop the model and a validation data set that is only used to evaluate the model developed with the training data. Therefore, models are evaluated with unseen data which guarantees an adequate performance for new data thus optimizing the usefulness of the model and preventing the model from over-fitting [126, 127].

Selected models were those that guaranteed an adequate performance for the validation data set. This guarantee was achieved by an early stopping of the learning process of the models using cross-validation [117]. More than 1,000 models for each data set were produced and tested by varying the possible values of the model and learning parameters. The learning rate was varied between 0.001 and 0.3 , the value of the momentum coefficient was varied between 0 and 0.5 , the number of hidden neurons was varied between 3 and 48 (only one hidden layer was sufficient to produce accurate results), and the standard deviation of the initial synaptic weights was varied between 0.05 and 1 (initial synaptic weights were produced randomly according to a Gaussian distribution). Moreover, a sufficiently high number of iterations $(5,000)$ was used in order to work with an early-stopping criterion for the learning process (Fig. 7.1). Training of the network was accomplished using the backpropagation learning algorithm [127] and the Expanded Range Approximation (ERA) algorithm, which proposes a variant to supervised learning algorithms in order to obtain the global minimum of the cost function (Section 6.3, Chapter 6).

\subsection{Results of the neural prediction}

For each subject, the pressure on fourteen foot anatomical points was recorded as well as the position of the sensors during a complete step. A similar pattern of the pressure curve measured by the same sensor during 
Chapter 7. Artificial Neural Networks for predicting dorsal pressures over the foot surface

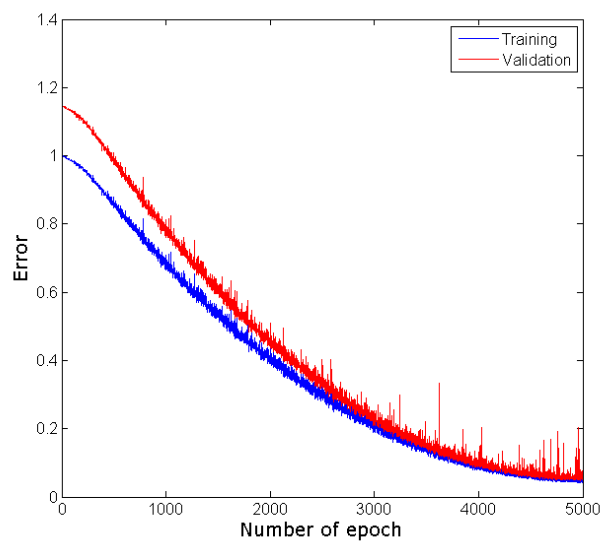

Figure 7.1: Error decay with the number of epoch for the validation and training processes.

the step was obtained for each of the materials, but with different values for the pressure due to the different parameters that characterize each material (Young's modulus, Poisson's ratio, and thickness). This showed the tendency of a subject to walk in the same way using the same design of shoe [44] (Section 4.3), thus suggesting the feasibility of obtaining a single model for all the materials.

Table 7.2: Results achieved by the best neural models in the training data set. Correlation coefficients are adimensional whereas all the error values (ME, MAE, $R M S E)$ are provided in $k P a$.

\begin{tabular}{|l|cccc|}
\hline \multirow{2}{*}{ Data set } & \multicolumn{4}{|c|}{ Training data set } \\
& ME & MAE & RMSE & r \\
\hline Subject \#1 (39) & -0.5010 & 3.5846 & 5.5409 & 0.9779 \\
Subject \#2 (42) & 0.1214 & 2.6912 & 4.2168 & 0.9702 \\
Subject \#3 (42) & -0.0179 & 1.9245 & 2.9540 & 0.9827 \\
Subject \#4 (42) & 0.1814 & 1.2953 & 2.0042 & 0.9836 \\
All subjects (33) & 0.2480 & 5.1860 & 8.6256 & 0.8959 \\
\hline
\end{tabular}


7.3. Results of the neural prediction

Table 7.3: Results achieved by the best neural models in the validation data set. Correlation coefficients are adimensional whereas all the error values (ME, MAE, RMSE) are provided in $k P a$.

\begin{tabular}{|l|cccc|}
\hline & \multicolumn{4}{|c|}{ Validation data set } \\
Data set & ME & MAE & RMSE & r \\
\hline Subject \#1 (39) & -0.0204 & 3.8423 & 5.7801 & 0.9791 \\
Subject \#2 (42) & 0.0139 & 2.9935 & 4.6365 & 0.9644 \\
Subject \#3 (42) & 0.0754 & 2.2381 & 3.5899 & 0.9757 \\
Subject \#4 (42) & 0.1960 & 1.4432 & 2.2863 & 0.9795 \\
All subjects (33) & 0.2735 & 5.3862 & 8.8002 & 0.8867 \\
\hline
\end{tabular}

Table 7.2 and Table 7.3 show the best results achieved in the prediction for the five data sets in training and validation, respectively. The number of hidden neurons corresponding to the best model is shown within parentheses for each data set. It should be pointed out that the best architecture was quite similar for the five data sets, which suggests that the mathematical model that performed the best prediction was similar for all the subjects. This is a relevant conclusion since an overall model that yields a fair prediction can be achieved beyond the subject-based model and even beyond the overall model for the four subjects and the five materials proposed in this work. The values of the following performance indices (see definitions in Section 6.4, Chapter 6) are shown in Tables 7.2 and 7.3.

- The Mean Error (ME) that is used as a measure of bias between the actual (desired) value of the pressure and the value predicted by the neural network.

- Mean Absolute Error (MAE) and Root Mean Square Error (RMSE), as measures of the accuracy.

- Correlation Coefficient (r), as a measure of the fit between the predicted pressure and the actual one. 
Chapter 7. Artificial Neural Networks for predicting dorsal pressures over the foot surface

One of the first conclusions that can be drawn from Tables 7.2 and 7.3 is the similar accuracy between training and validation, which indicates a balanced prediction. That is worthy of note since the validation data set was not used to train the model at all; therefore, the models obtained are able to provide predictions for unseen cases with roughly the same precision as that achieved in the training data set, which is made up of the patterns used to "teach" the model.

As it was already mentioned, the architectures of the different models are quite similar, which suggests that the underlying mathematical model should also be similar. That conclusions is corroborated when applying the model obtained for a given subject to another one; e.g., if the model obtained from Subject 1 data is applied to Subject 2, the correlation coefficient is 0.968 , very close to that obtained when applying the model obtained from the training data set of Subject 1 to the corresponding validation data set. This result shows up the generalization capability of the developed models that work appropriately with subjects different to those used to obtain the models. The same conclusion is reached when using a model developed from the information of some of the materials to a new one.

The interpretation of the correlation coefficient is straightforward since it is an adimensional index giving the similarity between the desired pressure and the predicted one. Tables 7.2 and 7.3 show that for subject-based models, correlation coefficients are higher than 0.96 (in both training and validation subsets). Therefore, the model is able to predict pressures that are very similar to the actual ones; it can be used as a reliable predictor of exerted pressures, and, in turn, as an indirect comfort predictor.

When working with the four subjects at the same time, the correlation coefficient decreases to 0.89 , which is still a very acceptable value. It is 
reasonable for $r$ to be lower in the overall data set than in the subjectbased data sets since dealing with the information of different subjects at the same time is more difficult. However, the main problem is that the number of subjects was very low (only four), which makes it difficult for the predictor to make an accurate prediction. If a higher number of subjects was available, the problem of the inter-individual variability would be drastically reduced because the neural network would have a wider range of scenarios from which draw overall conclusions.

The interpretation of the error values (ME, MAE, RMSE) is not that straightforward since these values provide errors measured in $\mathrm{kPa}$. The prediction capability of the neural network can be understood by analyzing the range of pressure values present in the data sets. Pressure values range between $1 \mathrm{kPa}$ and $376.6 \mathrm{kPa}$ (Section 4.3, Chapter 4), with a mean value of $19 \mathrm{kPa}$ and a standard deviation of $19 \mathrm{kPa}$. Therefore, the errors shown in Tables 7.2 and 7.3 correspond to very accurate models since MAE is approximately $10 \%$ of the mean value (and also of the standard deviation) of the pressures, being this result applicable to the different sensors; i.e., the wider the range of measured pressures for a give sensor, the higher the committed error. Fig. 7.2 shows the prediction provided by the neural model in two particularly challenging cases, in which abrupt peaks appear that make the modeling more complicated. Fig. 7.3 shows the prediction provided by the neural model for a case in which abrupt peaks does not appear.

\subsection{Discussion}

The predictions achieved are accurate since the correlation coefficients between the actual pressures and the predicted ones are higher than 0.96 for 
Chapter 7. Artificial Neural Networks for predicting dorsal pressures over the foot surface
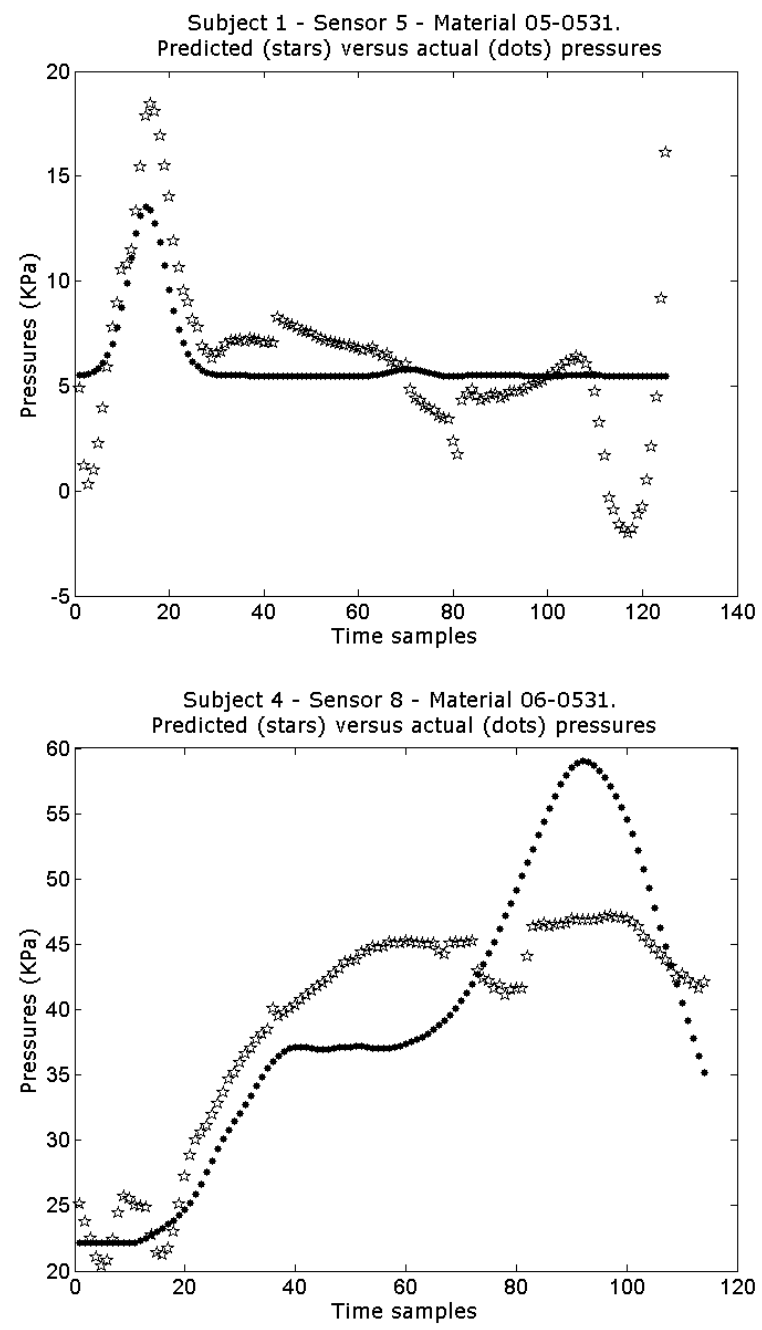

Figure 7.2: Experimental (dots) and predicted (stars) pressures for two particular challenging cases. Top: Subject 1, sensor 5, material MU05-0531. Bottom: Subject 4, sensor 8, material MU06-0531. 
7.4. Discussion

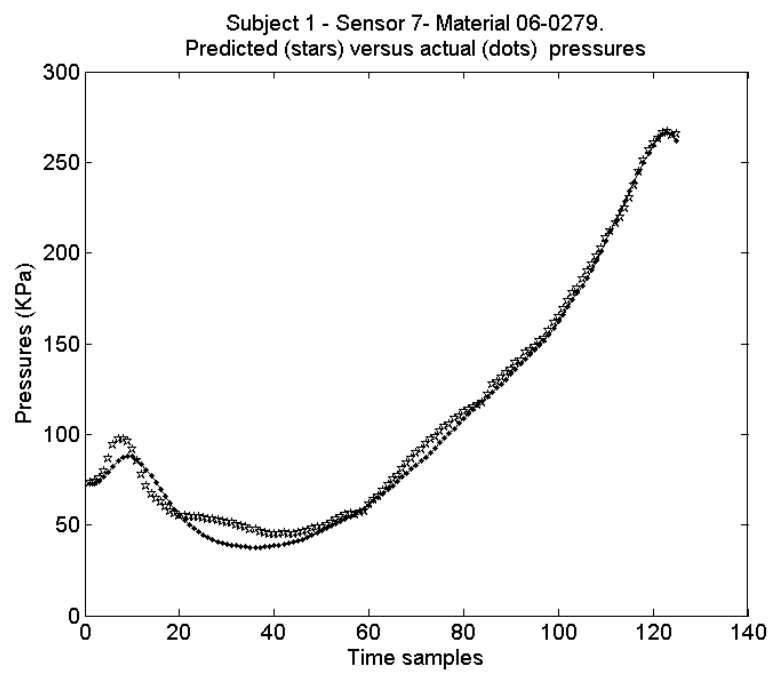

Figure 7.3: Experimental (dots) and predicted (stars) pressures for a particular case: Subject 1, sensor 7, material MU06-0279.

subject-based predictors and approximately 0.9 for an overall predictor. The measures of bias and accuracy have also shown that the proposed approach is a powerful tool for dealing with a reliable predictor that can be used to reduce the number of comfort tests during the footwear production process. For testing new shoes, it would be needed to prepare a biomechanical laboratory, specific sensors, anatomists to place sensors, technicians to manage the software, cameras, and later, professional staff for analyzing the data. However, this chapter has shown how ANNs can be used to obtain a simplified model to predict the exerted pressure for all the materials used as shoe uppers, thus decreasing the number of experiments to measure these pressures in the future.

Our ongoing research is focused on analyzing the most relevant features for prediction. This research can be used to determine whether some of 
Chapter 7. Artificial Neural Networks for predicting dorsal pressures over the foot surface

the six variables used for prediction can be withdrawn in order to simplify the predictor. It could also be used to reduce costs if some of the features measured were not necessary to achieve a sufficiently reliable pressure prediction.

To predict comfort in footwear, the information from the normal pressures should be complemented with the information about the shear forces. The shear forces in footwear are related to the rubbing and the sliding of the shoe with respect to the foot. No works that take into account the pressures exerted by these forces have been found in the literature, so that our ongoing research will also be focused on searching sensors able to measure these pressures on the foot surface in order to obtain their values to be added to the model.

As mentioned in Section 7.3, we feel that a larger data set made up of more subjects would enable us to obtain a more accurate overall predictor. Also, the training data set will be increased with new pressure data from shoes with different designs for each individual. It will then be possible to study the reliability of a more "generic" prediction model. Obtaining this kind of "generic" predictor for dorsal pressures for one individual would be crucial for many fields related to footwear, such as, Footwear Biomechanics, Footwear Industry, and Orthopedic Footwear.

\subsection{Summary}

In this chapter, the use of a predictor based on neural networks has been proposed for use in the selection of shoe upper materials. Given the properties of a material and the position of the sensors at any stage of the step, the pressure measured by the sensors for that material can be pre- 
dicted. This considerably reduces the time and cost involved in the analysis of pressures of this kind, which greatly influence footwear comfort [2]. It should also be emphasized that the inter-subject applicability of the model has been shown since models obtained using data from a certain subject work well when applying it to a different subject.

Our ongoing research is focused on a deeper study of feature relevance and the use of Fuzzy Logic in order to incorporate subjective information about comfort within the predictor. This way, we would be able to adapt the comfort prediction not just using pressures but how subjects experience comfort with different shoes, too. Also, we expect that a larger data set made up of more subjects would enable us to obtain a more accurate overall predictor. We are currently working on obtaining a larger data set with more subjects in order to verify this assumption. 
Chapter 7. Artificial Neural Networks for predicting dorsal pressures over the foot surface 


\section{Chapter 8}

\section{Conclusions}

\subsection{Virtual Reality and Finite Element Methods}

In this thesis, virtual reality and simulation techniques have been proposed as a viable alternative to the current procedures carried out to assess footwear. In Chapter 3, a prototype of a virtual simulator for footwear which performs virtual evaluations (in virtual environments) of the footwear features has been presented, Simucal. This virtual simulator is aimed at considerably reducing the temporal and economic costs involved in the development of a new footwear design.

Chapter 3 has also described a preliminary computational FE model that can be used to simulate the contact between the foot and the shoe upper as well as a procedure to automatically perform the initial alignment between the real scanned foot and any designed shoe. Although some of the assumptions have still been unavoidable in this first model, Simucal will provide a quantitative and visual analysis of the problems caused by footshoe interaction. This will allow the footwear manufacturers to virtually 
analyze the functionality of their footwear design.

It can be concluded that an animation of the foot that is reconstructed using the procedure presented in Chapter 4 can be introduced as the generic gait for a subject in the footwear virtual simulator since there does not exist any biomechanical model of the foot able to simulate its movements in a complete step. With this kind of animation, Simucal will provide in a future an evaluation of the problems caused by shoe-foot interaction while walking.

The results of Chapter 5 have shown how the behavior of the calfskin can be modeled with a linear orthotropic model when it is used as shoe upper material. However, some additional tests must still be designed aiming at determining the whole group of parameters that take part in the constitutive equation of this kind of leathers. New experiments have to be designed in order to validate whether this model is able to predict the shear forces at the contact areas. Moreover, a validation of the model for the whole foot-shoe upper interaction is needed.

\subsection{Artificial Neural Networks}

The research of this thesis is focused on developing the simulation of the contact between the foot and the shoe upper in a virtual way, so a fast characterization of the used materials is very useful to reduce costs. The aim of the tool developed in Chapter 6 is precisely that, to reduce the tests needed to characterize a material. Chapter 6 shows a procedure based on Artificial Neural Networks (ANNs) to reduce the number of flexibility tests that can be performed to evaluate the elasticity of the materials used as shoe uppers. Using the elastic parameters of the material as inputs 
to an ANN, it is possible to find a neural model that provides a single model for the relationship between force and displacement provided by these tests instead of a different characteristic curve for each material. Achieved results have shown the suitability of the novel proposed approach. The relevance of obtaining a simplified model for all the materials stems from the fact that this approach will model the behavior under contact of most of materials used as shoe uppers in footwear manufacture. It should be emphasized that this procedure can be applied to model the behavior under contact of other materials like for example, foams used as reinforcements or shoe counters, and hence, the contact with the foot surface of any kind of shoe upper could be modeled.

Chapter 7 has proposed the use of a predictor based on Artificial Neural Networks to predict the dorsal pressures on the foot surface while walking. Given the properties of the material and the position of the sensors at any stage of the step, the pressure measured by the sensors for that material can be predicted. This considerably reduces the time and cost involved in the analysis of pressures of this kind, which greatly influence footwear comfort. ANNs are used to obtain a single equation to model the exerted pressure for all the materials used as shoe uppers, thus decreasing the number of experiments necessary to measure these pressures in the future. Five different models have been produced, one model for each one of the four subjects (individuals) under study plus an overall model for the four subjects, and the achieved accuracy has been very satisfactory. It should also be emphasized that the inter-subject applicability of the model has been shown since models obtained using data from a certain subject work well when applying it to a different subject.

The main goal of predicting pressures on the foot surface is to indirectly predict the comfort in footwear. our ongoing research is focussed on the 
Chapter 8. Conclusions

use of Fuzzy Logic in order to incorporate subjective information about comfort within the predictor. This way, we would be able to adapt the comfort prediction not only using pressures but also the subjective opinions about the comfort of different users.

\subsection{Final conclusions}

The three techniques used in this thesis (Virtual Reality, Finite Element Methods, and Artificial Neural Networks) have been aimed at simulating the contact between the foot and the shoe upper in gait in order to predict the pressure exerted by the shoe on these areas. The final goal is the virtual assessment of footwear. Although very good results were obtained by the ANNs, this technique presents the disadvantage that the networks have to be previously trained. This involves to have previous data of the dorsal pressures exerted by some shoes on the foot surface of an individual from a biomechanical study of his/her performance in gait. Therefore, this technique is useful and fast when previous data of particular individuals have already been stored. The advantage of the FE modeling is that it is not needed previous knowledge about dorsal pressures, but a complete characterization of the materials that form the shoe is necessary. As it has been shown up, this is also a difficult task.

In summary, both techniques present benefits and drawbacks performing the dorsal pressure prediction. Therefore, future work must be carried out to improve these tools in order to combine both adding some additional techniques as Fuzzy Logic as mentioned before.

On the other hand, Virtual Reality has shown to be a very useful technique for the footwear assessment. Simucal, the developed footwear virtual sim- 
ulator, has been a good support for the FE model. In the near future, this virtual simulator will be adapted to support the ANN models too.

In conclusion, multidisciplinary techniques have been especially useful to solve the problem tackled in this thesis since the solutions to real problems, like the study of the foot-shoe upper interaction, usually need to come from multidisciplinary approaches. Virtual reality has been combined with FEM to build a virtual footwear simulator. ANNs have shown good capabilities to quickly characterize some materials to be used in the simulator. Even the pressures provided by the simulator could be validated with the pressures predicted by the developed neural predictor, thus minimizing the experimentation. Therefore, multidisciplinary techniques become a need and an especially valuable characteristic of this thesis.

\subsection{Future work}

The designed virtual simulator will be the basis for the development of new models for the footwear behavior. These models will be able to analyze a more realistic behavior of the materials using non-linear models and considering heterogeneous materials. This virtual simulator will also be adapted to run the ANNs models developed for the dorsal or other kind of pressure predictions.

Moreover, the procedure carried out to study the viability of obtaining a generic animation for the foot performance during the step will be extended to determine whether or not the gait of the subject is similar for any shoe to be worn.

Regarding the biomechanical models for leathers, our future work will carry on coping with all the problems found in the calfskin study until getting 
the best model for the simulation of leather behavior in gait. New experiments to completely determine all the parameters that take part in the constitutive equation of the model, as well as the validation of the model with other experiments that better represent the real contact between the shoe upper material and the foot upper surface will be carried out.

Another future work will be devoted to get the whole simulation of the shoe deformation in gait, including both upper and soles. Therefore, the procedure presented in chapter 6 will be applied to characterize most of the footwear components like reinforcements, insoles etc., in order to improve the development of a virtual tool that allow footwear manufacturers to analyze their designs in a much faster way.

Regarding neural prediction of pressures, our ongoing research is focused on a deeper study of feature relevance and the use of Fuzzy Logic in order to incorporate subjective information about comfort within the ANNs predictor. Also, we feel that a larger data set made up of more subjects would enable us to obtain a more accurate overall predictor. 


\section{Chapter 9}

\section{Conclusiones}

\subsection{Realidad Virtual y Elementos Finitos}

En esta Tesis se ha propuesto la realidad virtual y las técnicas de simulación como una alternativa viable a los actuales procedimientos que se llevan a cabo para evaluar el calzado. En el capítulo 3 se ha presentado Simucal, un prototipo de simulador virtual para el calzado que realiza evaluaciones virtuales (en entornos virtuales) de sus características funcionales, el cuál, tiene como objetivo reducir considerablemente los costes económicos y temporales que genera producir un nuevo diseño. En este capítulo se ha descrito también un modelo computacional de elementos finitos que puede ser utilizado para simular el contacto entre el pie y el corte del calzado, además de un procedimiento para ajustar automáticamente el pie al calzado en el instante inicial, y aunque algunas de las suposiciones llevadas a cabo para desarrollar esta aplicación son todavía inevitables, Simucal podrá proporcionar en un futuro análisis cuantitativos y visuales de los problemas causados por el calzado. 
A partir de los resultados obtenidos en el capítulo 4, se puede concluir también que una animación del pie durante el paso, que es reconstruida siguiendo el procedimiento presentado en este capítulo, puede ser usada como la forma de caminar genérica de un sujeto dado que no existe hasta la fecha ningún modelo biomecánico del pie que simule sus movimientos en un paso completo debido a su complejidad. Con esta animación, Simucal será capaz de evaluar los problemas causados por la interacción pie-zapato al caminar.

Los resultados del capítulo 5 han mostrado como el comportamiento del cuero de piel de becerro puede ser modelado con un modelo lineal ortotrópo cuando se utiliza como corte de calzado. Sin embargo, para determinar el conjunto completo de parámetros que definen la ecuación constitutiva de este tipo de cueros, se deberan diseñar experimentos adicionales. También se requeriran nuevos experimientos para validar si este modelo es capaz de predecir las fuerzas cortantes que actúan en las áreas de contacto, y para hacer una validación de si este modelo es capaz de simular la interacción completa entre el pie y el corte del calzado.

\subsection{Redes Neuronales Artificiales}

La investigación llevada a cabo en esta tesis tiene el propósito de simular el contacto entre el pie y el corte del calzado al caminar de modo virtual, de manera que una rápida caracterización de los materiales utilizados como cortes de calzado sería muy útil para reducir los costes asociados a diseñar un nuevo prototipo. El propósito de la herramienta desarrollada en el capítulo 6 es precísamente éste, reducir el número de experimentos llevados a cabo para caracterizar un material. El capítulo 6 muestra un procedimiento basado en Redes Neuronales Artificiales para reducir el 
número de tests de flexibilidad que se llevan a cabo para evaluar la elasticidad de los materiales usados como cortes de calzado. Con los parámetros elásticos del materiales como entrada a la red, es posible encontrar un modelo que proporciona una única ecuación para la relación entre la curva fuerza-desplazamiento proporcionada por estos tests, en lugar de llevar a cabo la experimentación para cada material evaluado. La relevancia de obtener un modelo simplificado para todos los materiales radica en el hecho de que este enfoque modelará el comportamiento bajo contacto de la mayoría de los materiales usados como corte de calzado. Además, se debe enfatizar que este procedimiento puede ser aplicado para modelar el comportamiento bajo contacto de otros componentes del calzado.

En el capítulo 7 se propone el uso de un predictor basado también en redes neuronales para predecir las presiones dorsales en la superfice del pie mientras se camina. Dadas las propiedades del material y la posición de un sensor en cualquier etapa del paso, se puede predecir la presión ejercida por el material. Ésto reduce considerablemente el coste temporal y económico que implicaría medir este tipo de presiones para cada usuario y con cada zapato. Las redes neuronales artificiales proporcionan una única ecuación que modela la presión ejercida por todos los materiales usados como cortes de calzado, de este modo se minimizará la cantidad de experimentos necesarios para medir estas presiones en un futuro.

Se han producido cinco modelos diferentes, uno para cada uno de los sujetos y uno global para los cuatro sujetos bajo estudio. La exactitud de los modelos ha sido muy satisfactoria incluso se ha demostrado la aplicabilidad de dichos modelos entre sujetos ya que el modelo obtenido para un sujeto es aplicable al resto de sujetos.

El principal objetivo de predecir las presiones dorsales (presiones en la superficie del pie) es predecir de manera indirecta el confort en el calzado. 
Nuestro próximo objetivo es el uso de la Lógica Difusa para incorporar la información subjetiva sobre el confort que proporcionan los individuos dentro del predictor, de este modo seremos capaces de adaptar la predicción, no sólo usando presiones sino también las opiniones subjetivas de los usuarios.

\subsection{Conclusiones finales}

Las tres técnicas presentadas en esta tesis: Realidad Virtual, el Método de los Elementos Finitos y las Redes Neuronales Artificiales tienen el propósito de simular el contacto entre el pie y el corte del calzado al caminar con el fin de predecir las presiones en las áreas de contacto, y el objetivo final es la evaluación virtual del calzado. Aunque se han obtenido muy buenos resultados con las Redes Neuronales Artificiales, esta técnica presenta la desventaja de que las redes han de ser previamente entrenadas, lo que implica tener datos previos de las presiones ejercidas por algunos zapatos en la superficie del pie del individuo que camina, las cuales procederán de estudios biomecánicos de su forma de caminar. Por tanto, esta técnica es útil y rápida cuando se tienen estos datos previos. La ventaja de modelar con los elementos finitos es que no se necesita conocimiento previo de presiones dorsales, sino una caracterización completa de los materiales que forman el zapato. Como hemos mostrado en esta tesis, esto no es tarea fácil.

En resumen, ambas técnicas presentan ventajas e incovenientes a la hora de realizar la predicción de las presiones dorsales. Por tanto, será necesario profundizar más en estas técnicas y ver si es posible combinarlas, para conseguir la mejor herramienta posible para la predicción del confort en el calzado. 
Por otro lado, la realidad virtual ha mostrado ser una técnica muy útil para la evaluación del calzado. El simulador desarrollado, Simucal, ha sido un buen soporte para el modelo de elementos finitos. En un futuro cercano, este simulador podría también ser soporte para los modelos propuestos basados en redes neuronales.

En conclusión, las técnicas multidisciplinares han sido especialmente útiles para resolver el problema al que nos enfrentábamos en esta tesis ya que los propblemas reales como éste necesitan muchas veces de enfoques multidisciplinares. La Realidad Virtual ha sido combinada con los Elementos Finitos para construir un simulador virtual para el calzado. Las Redes Neuronales Artificiales han mostrado buenas capacidades para modelar rápidamente algunos materiales usados en el simulador. Incluso, las presiones proporcionadas por el simulador podrían ser validadas con las presiones proporcionadas por el predictor neuronal desarrollado, minimizando de este modo la experimentación. Las técnicas multidisciplinares se convierten así en una necesidad y una característica especialmente valiosa de esta tesis.

\subsection{Trabajos futuros}

El simulador virtual diseñado será la base para el desarrollo de nuevos modelos de comportamiento para el calzado. Estos modelos serán capaces de analizar un comportamiento más realista de los materiales no lineales y considerando materiales hetereogéneos. Este simulador virtual también será adaptado para lanzar los modelos de redes neuronales desarrollados para la predicción de las presiones dorsales o de cualquier tipo de presiones.

Además, el procedimiento llevado a cabo para estudiar la viabilidad de 
obtener una animación genérica del movimiento del pie al caminar se extenderá para determinar si la forma de andar de un sujeto es la misma independientemente del zapato que lleve.

Respecto al modelo biomecánico del corte del calzado, nuestro trabajo futuro se centrará en solucionar los problemas encontrados en la completa caracterización del cuero de piel de becerro con nuevos experimentos y nuevos modelos.

Otros trabajos futuros intentarán conseguir la simulación del comportamiento de todo el zapato al caminar, ésto incluirá corte y suela. Por tanto, el procedimiento desarrollado en el capítulo 6 será extendido para caracterizar la mayoría de los componentes del calzado con el fin de que los fabricantes analicen sus diseños de forma rápida con la herramienta desarrollada.

Respecto a la predicción de las redes neuronales, nuestro trabajo futuro se centrará en un trabajo más profundo sobre la relevancia de las distintas variables de entrada al modelo y en como introducir la información subjetiva sobre el confort mendiante, por ejemplo, lógica difusa. Nuestro trabajo futuro se centrará también en obtener más presiones de más individuos con más tipos de zapatos con el fin de mejorar la calidad del predictor global desarrollado. 


\section{Appendix I: The UMAT subroutine for the Lin \& Hayhurst model}

C

SUBROUTINE UMAT (STRESS, STATEV , DDSDDE, SSE, SPD , SCD ,

1 RPL, DDSDDT , DRPLDE, DRPLDT ,

2 STRAN, DSTRAN , TIME, DTIME, TEMP , DTEMP , PREDEF, DPRED , CMNAME,

3 NDI , NSHR, NTENS, NSTATEV , PROPS , NPROPS , COORDS , DROT , PNEWDT,

4 CELENT, DFGRD0, DFGRD1, NOEL, NPT, LAYER, KSPT , KSTEP , KINC)

C

INCLUDE 'ABA_PARAM. INC'

C

CHARACTER $* 8$ CMNAME

DIMENSION STRESS (NTENS), STATEV (NSTATEV),

1 DDSDDE (NTENS, NTENS), DDSDDT (NTENS), DRPLDE (NTENS),

2 STRAN (NTENS), DSTRAN (NTENS), TIME (2), PREDEF (1), DPRED (1),

3 PROPS (NPROPS), COORDS (3), DROT $(3,3), \operatorname{DFGRDO}(3,3), \operatorname{DFGRD} 1(3,3)$

$\mathrm{C}$

C LOCAL ARRAYS

C RCGDT - RIGHT CAUCHY-GREEN DEFORMATION TENSOR

C LST - LAGRANGIAN (OR GREEN) STRAIN TENSOR

C KST - KIRCHHOFF STRESS TENSOR

C SFT - PRODUCT OF $\mathrm{S} * \mathrm{Ft}$

C-

$\mathrm{C}$

REAL RCGDT, LST, KST, SFT

C

DIMENSION RCGDT (6), $\operatorname{LST}(6), \operatorname{KST}(6), \operatorname{SFT}(3,3)$

c

PARAMETER $(Z E R O=0 . \mathrm{DO}$, ONE $=1 . \mathrm{DO}, \mathrm{HALF}=0.5 \mathrm{DO})$

C

MATERIAL PROPERTIES 
C

$$
\begin{aligned}
\text { A11 } & =\operatorname{PROPS}(1) \\
\text { A22 } & =\operatorname{PROPS}(2) \\
\text { A33 } & =\operatorname{PROPS}(3) \\
\text { A12 } & =\operatorname{PROPS}(4) \\
\text { A23 } & =\operatorname{PROPS}(5) \\
\text { A13 } & =\operatorname{PROPS}(6) \\
\text { A77 } & =\operatorname{PROPS}(7)
\end{aligned}
$$

$\mathrm{C}$

$\mathrm{C}$

$\mathrm{C}$

C

JACOBIAN OF THE DEFORMATION TENSOR

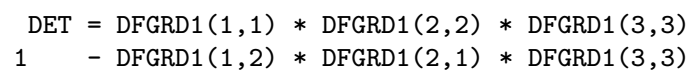




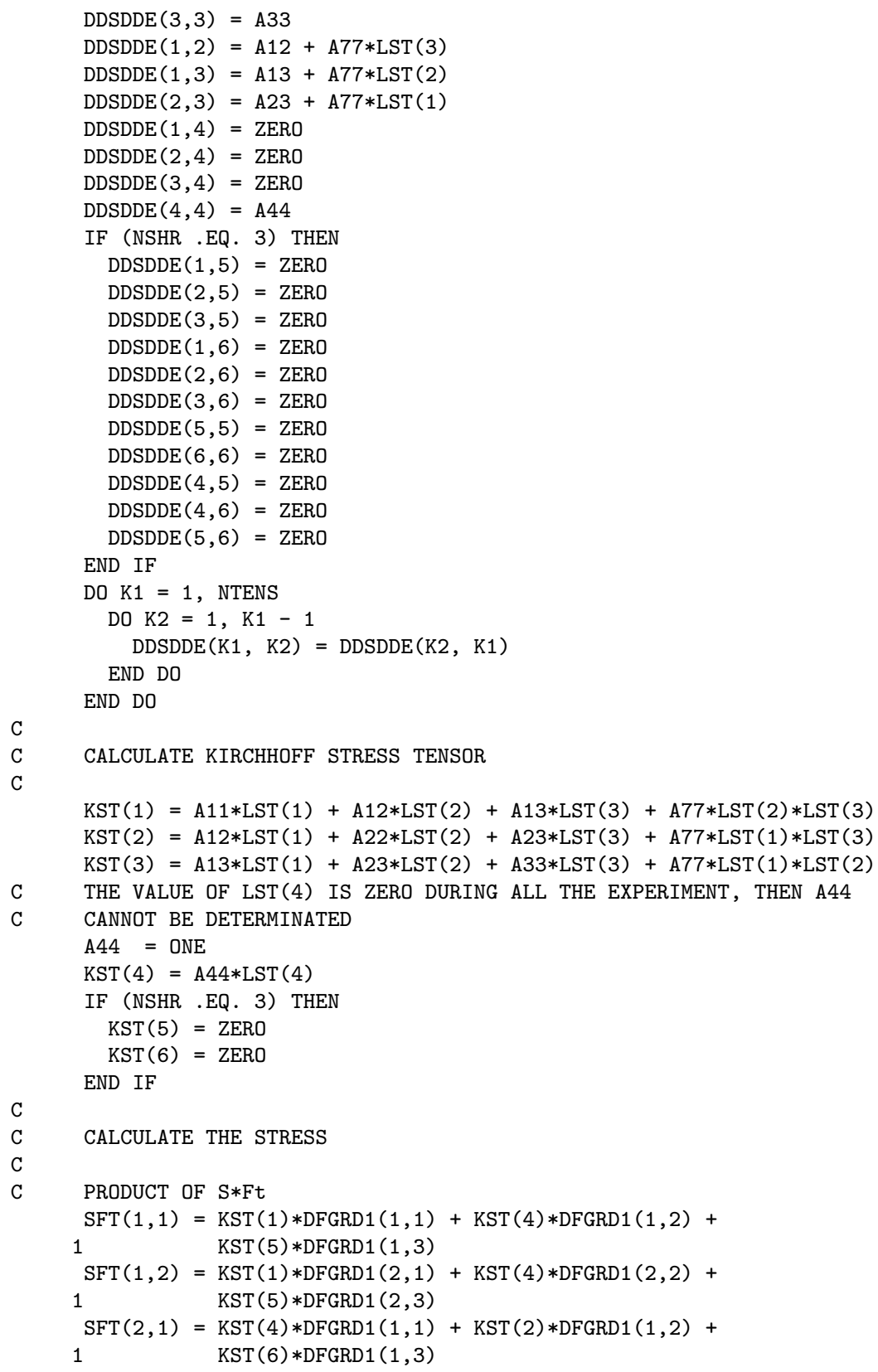




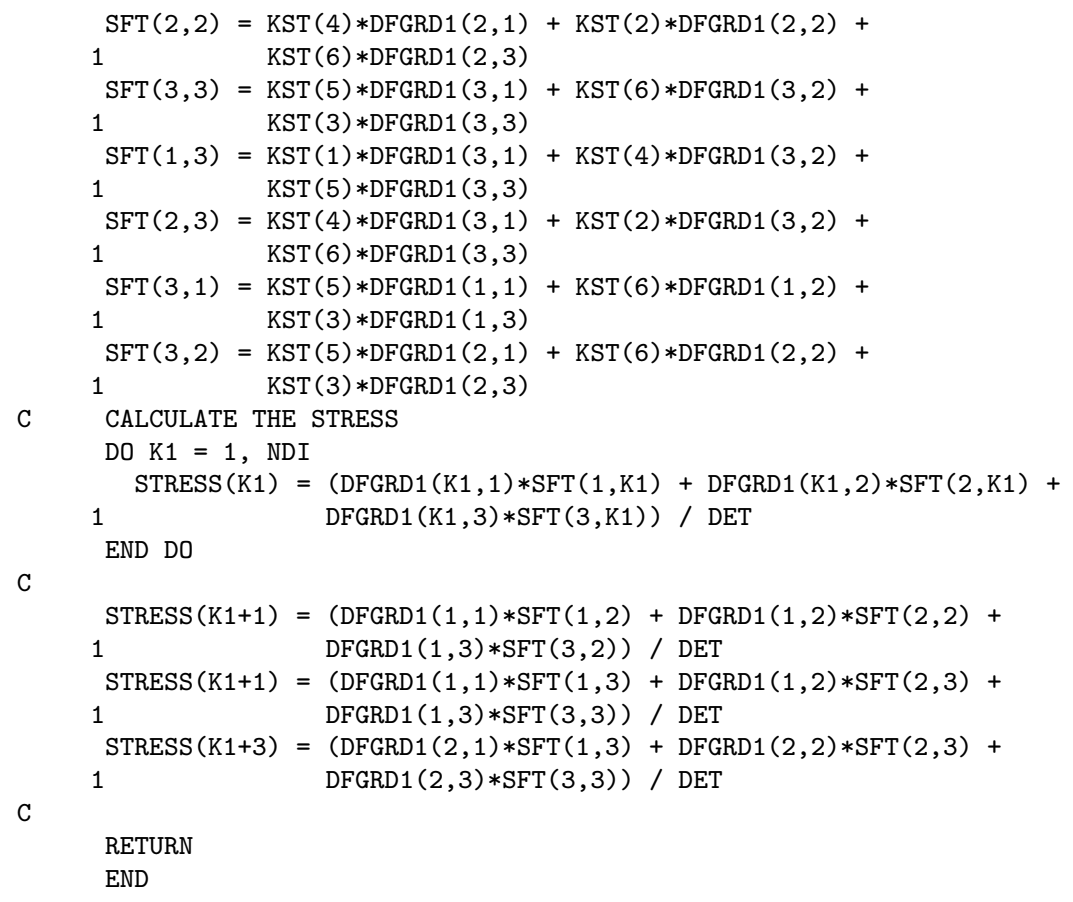




\section{List of Figures}

1.1 Work flow of computer-aided engineering approach for footwear design proposed by Cheung et al. in [1]. . . . . . . . . 3

1.2 Building blocks of the proposed work flow for a footwear ICAD system. . . . . . . . . . . . . . . . 4 4

2.1 The 2D Lemmon's finite element model. Image from [12]. . 14

2.2 A schematic illustration of a plug inserted in the midsole of

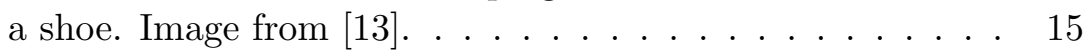

2.3 Axisymmetric mesh used for FE analysis of stud impacts on protected ankle. Image from $[15] \ldots \ldots . . . . . .16$

2.4 Goske's plane strain finite element model. Image from [17]. 18

2.5 Actis' foot model. Image from [19]. . . . . . . . . . . 20

2.6 Chu's foot, ankle, and orthosis model. Image from [21]. . . 21

2.7 (A) Finite element models of the foot bones with cartilage and major plantar ligaments, and (B) the complete model of the foot with soft tissues [32]. . . . . . . . . 26

2.8 (A) Finite element models of the flat insole (consisted of Microcel Puff), (B) TCI-1 (consisted of PPT, Microcel Puff and Thermocork), and (C) TCI-2 (consisted of medium Plastazote and PPT) [32] . . . . . . . . . . . . . 26 
2.9 (A) The finite element mesh of soft tissues, (B) bony and ligamentous structures, and (C) flat and custom-molded insoles. Figure from [34]. . . . . . . . . . . . . . . 27

2.10 A typical graph of all curves of a patient normalized to the gait cycle (horizontal axis) and into the range $0-1$ (vertical axis) using the clinical ranges of angles, moments and powers (The curves with values above one or below zero had values off the scale on the conventional plots). Image from $[64]$

2.11 (a) Results of six patients whose SOM curves run through a cluster of nodes surrounded by the square. (b) The histogram shows the number of times each node was activated by the six curves. Image from [64] . . . . . . . . . . . . . 34

3.1 Software program structure developed for the simulator. . . 40

3.2 Foot, upper and sole meshes. . . . . . . . . . . . . . 40

3.3 Membrane loading and bending on a plane element. . . . . 46

3.4 Plane triangular element in local coordinates. . . . . . . . . 49

3.5 Area coordinates. . . . . . . . . . . . . . . . 53

3.6 Nodes of the upper mesh inside the foot mesh in frame 0 (left). Distribution of forces normal to the upper surface in frame 0 (right). . . . . . . . . . . . . . 64

3.7 Detection of minimum $x$ coordinate point and middle axis for the shoe (top) and for the foot (bottom). Image from IBV (Institute of Biomechanics of Valencia). . . . . . . . 67

3.8 Initial foot-shoe alignment. . . . . . . . . . . . . 68

3.9 Points from a square shell that are inside a sphere, which are placed on the sphere surface with an algorithm that considers the sphere as an enclosed surface and looks for the closest point on the sphere surface to the node of the square shell. . . . . . . . . . . . . . . . 70 
3.10 Clamped plate with a point load at the center (top) and pinched hemisphere (bottom). . . . . . . . . . . 71

3.11 Pinched cylinder (top) and the Scordelis-Lo roof (bottom). 72

3.12 Validation of the elastic model with a clamped plate, with the pinched cylinder, with the pinched hemisphere, and with the Scordelis-Lo roof. . . . . . . . . . . . . . . . 73

3.13 Tensile test results for three leathers used as shoe uppers in this work. . . . . . . . . . . . 77

3.14 Meshes for the foot, for the shoe upper, and for the shoe sole. 79

3.15 Simulation of the initial fit between the shoe and the foot for material I where the normal force distribution is shown in colors in the images at the bottom of the figure. The initial screen of the user interface is shown in the top left image, and the second screen of the user interface is shown in the top right image. . . . . . . . . . . . 81

4.1 3D static foot form record. Image from IBV. . . . . . . 90

4.2 Key anatomic landmarks (Image from Infoot de I-ware Lab-

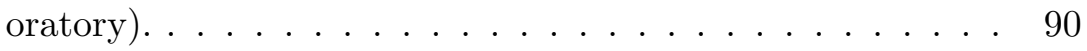

4.3 Foot movement analysis system. Image from IBV . . . . . . 91

4.4 Foot animation. . . . . . . . . . . . . . . . . . . 93

4.5 Kind of shoe used in the experiments. . . . . . . . . . 94

4.6 Distribution of the pressure sensors on the foot surface. Image from IBV. . . . . . . . . . . . . . . . . . . . 94

4.7 Peak pressure distributions along a complete step for all the subjects on all the sensors. . . . . . . . . . . 96

4.8 Dorsal pressure distribution for subject 1, sensor 1 to sensor $6 \ldots \ldots \ldots \ldots \ldots \ldots \ldots \ldots$

4.9 Dorsal pressure distribution for subject 1, sensor 7 to sensor $12 \ldots \ldots \ldots \ldots \ldots \ldots \ldots \ldots$ 
4.10 Dorsal pressure distribution for subject 1, sensors 13 and 14. 99

4.11 Dorsal pressure distribution for subject 3 on sensor 1 to sensor 4. . . . . . . . . . . . . . 100

4.12 Dorsal pressure distribution for subject 3 sensor 5 to sensor $10 \ldots \ldots \ldots \ldots \ldots 10 \ldots \ldots$

4.13 Dorsal pressure distribution for subject 3 sensor 11 to sensor 14. . . . . . . . . . . . . . . 102

4.14 Dorsal pressure distributions on sensor 10 for each subject. 103

5.1 Schematic diagram of the structure of leather before (a) and after (b) tanning process. Figure from [104]. . . . . . . . . 111

5.2 Shape and dimensions of uniaxial tensile test specimen. Dimensions in mm (figure from [104]). . . . . . . . . . . 115

5.3 Calfskin (left). Universal testing machine (right). . . . . . . 115

5.4 Test to measure the resistance to damage on lasting. . . . . 116

5.5 Simulation of the resistance to damage on lasting using ANSYS . . . . . . . . . . . . . . . 117

5.6 The shape and size of specimens for cylindrical last forming tests. All dimensions are in mm. . . . . . . . . . . . . . . 118

5.7 Simulation of the shoe forming process with shoe lasts. . . . 119

5.8 Simulation of the last forming test using ANSYS. . . . . . . 120

5.9 Tensile stress versus axial strain for shoulder (top), butt (middle), and belly (bottom) areas of skin 1. . . . . . . 121

5.10 Tensile stress versus axial strain for shoulder (top), butt (middle), and belly (bottom) areas of skin 2. . . . . . . 122

5.11 Stress versus strain from shoulder (top), butt (middle), and belly (bottom) areas of skin 1 . Results of the tensile test following the standard UNE-EN-ISO 3376 . . . . . . . . 124 
5.12 Stress versus strain from shoulder (top), butt (middle), and belly (bottom) areas of skin 2. Results of the tensile test following the standard UNE-EN-ISO 3376 . . . . . . . 125

5.13 Lateral strain versus axial elongation for shoulder (top), butt (middle), and belly (bottom) areas of skin 1. . . . . . 127

5.14 Lateral strain versus axial elongation for shoulder (top), butt (middle), and belly (bottom) areas of skin 2. . . . . 128

5.15 Deformation of the calfskin sample. . . . . . . . . . . . 129

5.16 Example of orthotropic material. . . . . . . . . . . 132

5.17 Load versus displacement of the central point of the samples cut in shoulder, butt and belly areas of skin 1 (top left) and of skin 2 (top right). Stress versus strain for the shoulder, butt and belly areas of skin 1 (bottom left) and of skin 2 (bottom right). PD and PL mean perpendicular and parallel to the backbone respectively. . . . . . . . . . . . . 137

5.18 Simulation of the resistance to damage on lasting using ANSYS for the shoulder area of skin 1. Deformed mesh (left) and displacements (right). . . . . . . . . . . . . 138

5.19 Force versus displacement of the central point of the sample in the simulation of resistance to damage on lasting for samples cut in the shoulder (top), butt (middle), and belly (bottom) areas of calfskin 1. . . . . . . . . . . . . . 139

5.20 Force versus displacement of the central point of the sample in the simulation of resistance to damage on lasting for samples cut in the shoulder (top), butt (middle), and belly (bottom) areas of calfskin 2. . . . . . . . . . . . 140

5.21 Simulation of the last forming test using ANSYS for the butt area of skin 2. Deformed mesh (left) and displacements (right) . . . . . . . . . . . . . . . . . 141 
5.22 Force versus displacement of the central point of the sample in the simulation of the last forming tests for samples cut in the shoulder (top), butt (middle), and belly (bottom) areas of the calfskin $1 . \ldots \ldots \ldots$. . . . . . . . . 142

5.23 Force versus displacement of the central point of the sample in the simulation of the last forming tests for samples cut in the shoulder (top), butt (middle), and belly (bottom) areas of the calfskin $2 . \ldots \ldots \ldots \ldots$

6.1 Flexibility test (left). Force versus material displacement (right). Image from IBV. . . . . . . . . . . . . . 149

6.2 Schematic diagram of a neural model. Image from [49]. . . 151

6.3 Schematic diagram of the most used neuron model. . . . . . 153

6.4 Multilayer perceptron. Dotted arrows stand for recurrent neural systems. Image from [49]. . . . . . . . . . . . . 155

6.5 Force versus displacement for the three material under study.169

6.6 Error decay with the number of epoch for the validation and training processes. . . . . . . . . . . . 173

6.7 Neural network modeling (circles) and real values of force (stars) for approximately third of the patterns belonging to the validation data set (random selection of patterns) $\ldots 176$

7.1 Error decay with the number of epoch for the validation and training processes. . . . . . . . . . . . . 184

7.2 Experimental (dots) and predicted (stars) pressures for two particular challenging cases. Top: Subject 1, sensor 5, material MU05-0531. Bottom: Subject 4, sensor 8, material MU06-0531. . . . . . . . . . . . . . 188

7.3 Experimental (dots) and predicted (stars) pressures for a particular case: Subject 1, sensor 7, material MU06-0279. . 189 


\section{List of Tables}

3.1 Data of the material used as shoe sole. . . . . . . . . . 65

3.2 Data of the tested meshes (S.I.). . . . . . . . . . 74

3.3 Data of the leathers used as shoe uppers. . . . . . . . 78

4.1 Mechanical properties of the leathers used as shoe upper materials. Data provided by the IBV. . . . . . . . . . 95

5.1 Young's moduli for calfskin 1. . . . . . . . . . . . . 134

5.2 Poisson's ratios for calfskin 1. . . . . . . . . . . . . 134

5.3 Shear moduli for calfskin 1. . . . . . . . . . . . . . . . 134

5.4 Young's moduli for calfskin 2. . . . . . . . . . . . . . . 134

5.5 Poisson's ratios for calfskin 2. . . . . . . . . . . 135

5.6 Shear moduli for calfskin $2 \ldots \ldots \ldots$

6.1 Most used cost functions . . . . . . . . . . . . . . . . 157

6.2 Data of the materials used in the flexibility tests. . . . . . 168

6.3 Results achieved in the MLP modeling of the exerted force to the sphere. All values are expressed in Newtons. . . . . . 175

7.1 Number of records (time samples) for the different data sets. 182 
7.2 Results achieved by the best neural models in the training data set. Correlation coefficients are adimensional whereas all the error values (ME, MAE, RMSE) are provided in $\mathrm{kPa} .184$

7.3 Results achieved by the best neural models in the validation data set. Correlation coefficients are adimensional whereas all the error values (ME, MAE, RMSE) are provided in $\mathrm{kPa} .185$ 


\section{Bibliography}

[1] J. T. Cheung, Y. Jia, D. Wai-Chi, and M. Zhang, "Current methods in Computer-Aided Engineering for footwear design," Footwear Science, vol. 1, no. 1, pp. 31-46, 2009.

[2] C. Jordan and R. Bartlett, "Pressure distribution and perceived comfort in casual footwear," Gait and Posture, vol. 3, no. 4, pp. 215-220, 1995.

[3] P. Azariadis, V. Moulianitis, S. Alemany, J. C. González, P. de Jong, M. van der Zande, and D. Brands, "Virtual shoe test bed: A computeraided engineering tool for supporting shoe design," Computer-Aided Design E Applications, vol. 4, no. 6, pp. 741-750, 2007.

[4] S. G. Papageorgiou, V. C. Moulianitis, and N. A. Aspragathos, "Transfer VM concepts to cloth design and manufacturing," In: Proceedings of Eurasia-Tex Conference on 3D Body Scanning and Virtual Try-On Systems, Athens, Greece, pp. 41-49, 2003.

[5] W. I. Schöllhorn, "Applications of artificial neural nets in Clinical Biomechanics," Clinical Biomechanics, vol. 19, no. 9, pp. 876-898, 2004.

[6] M. J. Rupérez, C. Monserrat, M. Alcañiz, S. Alemany, and S. Puigcerver, "Footwear virtual simulator for the analysis of comfort and functionality in new designs of shoes," In: Proceedings of the 8th Footwear Biomechanics Symposium. Frederick E. C., Yang, S.W., (Eds.). Taipei, Taiwan, pp. 9192, 2007.

[7] T. J. Hwang, K. Lee, H. Y. Oh, and J. H. Jeong, "Derivation of template shoe-lasts for efficient fabrication of customordered shoe-lasts," ComputerAided Design, vol. 37, no. 12, pp. 1241-1250, 2005. 
[8] L. Kos and J. Donovnik, "A system for footwear fitting analysis," In: Proceedings of the Intenational Design Conference-Design, Dubrovnik, pp. 1187-1192, 2002.

[9] C. P. Witana, J. Feng, and R. S. Goonetilleke, "Dimensional differences for evaluating the quality of footwear fit," Ergonomics, vol. 47, no. 12, pp. 1301-1317, 2004.

[10] M. J. Rupérez, C. Monserrat, S. Alemany, M. C. Juan, and M. Alcañiz, "Contact model, fit process and, foot animation for the virtual simulator of the footwear comfort," Computer-Aided Design, vol. 42, no. 5, pp. 425-431, 2010 .

[11] S. Nakamura, R. O. Crowninshield, and R. R. Cooper, "An analysis of soft tissue loading in the foot - A preliminary report," Bulletin of Prosthetics Research, vol. 18, pp. 27-34, 1981.

[12] D. Lemmon, T. Y. Shiang, A. Hashmi, J. S. Ulbrecht, and P. R. Cavanagh, "The effect of insoles in therapeutic footwear - A finite element approach," Journal of Biomechanics, vol. 30, no. 6, pp. 615-620, 1997.

[13] A. Erdemir, J. J. Saucerman, D. Lemmon, B. Loppnow, B. Turso, J. S. Ulbrecht, and P. R. Cavanagh, "Local plantar pressure relief in therapeutic footwear: Design guidelines from finite element models," Journal of Biomechanics, vol. 38, no. 9, pp. 1798-1806, 2005.

[14] M. R. Shorten, "Finite element modeling of soccer shoe soles," In: Proceedings of World Cup Scientific Congress, Lyons, France, 1998.

[15] S. Ankrah and N. I. Mills, "Analysis of ankle protection in association football," Sports Engineering, vol. 7, no. 1, pp. 41-52, 2004.

[16] R. Verdejo and N. J. Mills, "Heel-shoe interactions and the durability of EVA foam running-shoe midsoles," Journal of Biomechanics, vol. 37, no. 9, pp. 1379-1386, 2004.

[17] S. Goske, A. Erdemir, M. Petre, S. Budhabhatti, and P. R. Cavanagh, "Reduction of plantar heel pressures: Insole design using finite element analysis," Journal of Biomechanics, vol. 39, no. 13, pp. 2363-2370, 2006.

[18] I. R. Spears, J. E. Miller-Young, J. Sharma, R. F. Ker, and F. W. Smith, "The potential influence of the heel counter on internal stress during static standing: A combined finite element and positional MRI investigation," Journal of Biomechanics, vol. 40, no. 12, pp. 2774-2780, 2007. 
[19] R. L. Actis, L. B. Ventura, K. E. Smith, P. K. Commean, D. J. Lott, T. K. Pilgram, and M. J. Mueller, "Numerical simulation of the plantar pressure distribution in the diabetic foot during the pushoff stance," Medical and Biological Engineering and Computing, vol. 44, no. 8, pp. 653-663, 2006.

[20] R. L. Actis, L. B. Ventura, D. J. Lott, K. E. Smith, P. K. Commean, M. K. Hastings, and M. J. Mueller, "Multi-plug insole design to reduce peak plantar pressure on the diabetic foot during walking," Medical and Biological Engineering and Computing, vol. 46, no. 4, pp. 363-371, 2008.

[21] T. M. Chu, N. P. Reddy, and J. Padovan, "Three-dimensional finite element stress analysis of the polypropylene, ankle-foot orthosis: Static analysis," Medical Engineering and Physics, vol. 17, no. 5, pp. 372-379, 1995.

[22] S. Syngellakis, M. A. Arnold, and R. Rassoulian, "Assessment of the nonlinear behaviour of plastic ankle foot orthoses by the finite element method," In: Proceedings of the Institution of Mechanical Engineers, Part H, vol. 214, no. 5, pp. 527-539, 2000.

[23] M. R. Shorten, "Biomechanically driven developments in sport shoe technology: The example of tuned air," In: Proceedings of the 4th Symposium on Footwear Biomechanics, Canmore, Canada, 1999.

[24] G. Baroud, U. J. Goerke, H. Guenther, D. J. Stefanyshyn, J. E. Miller, and B. M. Nigg, "A non-linear hyperelastic finite element model of energy return enhancement in sport surfaces and shoes," In: Proceedings of the 4th Symposium on Footwear Biomechanics, Canmore, Canada, pp. 18-19, 1999.

[25] T. Y. Shiang, "The nonlinear finite element analysis and plantar pressure measurement for various shoe soles in heel region," In: Proceedings of the National Science Council, Republic of China, Part B, vol. 21, no. 4, pp. 168$174,1997$.

[26] N. Even-Tzur, E. Weisz, Y. Hirsch-Falk, and A. Gefen, "Role of EVA viscoelastic properties in the protective performance of a sport shoe: Computational studies," Bio-Medical Materials and Engineering, vol. 16, no. 5, pp. 289-299, 2006.

[27] S. Alemany, I. García, E. Alcántara, J. C. González, and L. Castillo, "Integration of plantar pressure measurements in a finite element model for the optimization of shankpiece design for high-heeled shoes," In: Proceedings of the 6th Symposium on Foolwear Biomechanics, Queenstown, New Zealand, 2003. 
[28] Z. Sun, D. Howard, and M. Moatamedi, "Finite element analysis of footwear and ground interaction," Strain, vol. 41, pp. 113-117, 2005.

[29] Z. Barani, M. Haghpanahi, and H. Katoozian, "Three dimensional stress analysis of diabetic insole: A finite element approach," Technology and Health Care, vol. 13, no. 3, pp. 185-192, 2005.

[30] T. Nishiwaki, "Running shoe sole stiffness evaluation method based on eigen vibration analysis," Sports Technology, vol. 1, no. 1, pp. 76-82, 2008.

[31] T. Oda, K. Oomori, and Y. Kaneko, "Running shoes development using a finite element ankle-joint model," In: Proceedings of the 6th Symposium on Footwear Biomechanics, Queenstown, New Zealand, 2003.

[32] W. P. Chen, C. W. Ju, and F. T. Tang, "Effects of total contact insoles on the plantar stress redistribution: A finite element analysis," Clinical Biomechanics, vol. 18, no. 6, pp. 17-24, 2003.

[33] S. C. Lin, C. H. Lin, F. T. Tang, and W. P. Chen, "Combining experimental material property test and finite element analysis to investigate the plantar foot pressure distribution during standing," In: Proceedings of 21st Congress of the Internalional Society of Biomechanics, Taipei, Taiwan. Journal of Biomechanics, vol. 40, no. S2, pp. 337-338, 2007.

[34] J. T. Cheung and M. Zhang, "A 3-dimensional finite element model of the human foot and ankle for insole design," Archives of Physical Medicine and Rehabilitation, vol. 86, no. 2, pp. 353-358, 2005.

[35] J. T. Cheung and M. Zhang, "Parametric design of pressure-relieving foot orthosis using statistics-based finite element method," Medical Engineering and Physics, vol. 30, no. 3, pp. 269-277, 2008.

[36] J. T. Cheung and M. Zhang, "A 3D finite element simulation of foot-shoe interface," In: Proceedings of the 8th Symposium on Footwear Biomechanics, Taipei, Taiwan, pp. 45-46, 2007.

[37] J. Yu, J. Cheung, Y. Z. Y. Fan, A. Leung, and M. Zhang, "Development of a finite element model of female foot for high-heeled shoe design," Clinical Biomechanics, vol. 23, no. S1, 2008.

[38] S. P. Budhabhatti, A. Erdemir, M. Petre, J. Sferra, B. Donley, and P. R. Cavanagh, "Finite element modeling of the first ray of the foot: A tool for the design of interventions," Journal of Biomechanical Engineering, vol. 129, no. 5, pp. 750-756, 2007. 
[39] Y. C. Hsu, Y. W. Gung, S. L. Shih, C. K. Feng, S. H. Wei, C. H. Yu, and C. S. Chen, "Using an optimization approach to design an insole for lowering plantar fascia stress - A finite element study," Annals of Biomedical Engineering, vol. 36, pp. 1345-1352, 2008.

[40] P. J. Antunes, G. R. Dias, A. T. Coelho, F. Rebelo, and T. Pereira, "Hyperelastic modelling of corkpolyurethane gel composites: Non-linear FEA implementation in 3D foot model," Malerials Science Forum, vol. 587-588, pp. 700-705, 2008.

[41] F. Cui, R. P. Lee, and X. Zeng, "Impact analysis of shoes using the structural intensity technique," In: Proceedings of 4 th European Conference of the International Federation for Medical and Biological Engineering, Antwerp, Belgium, pp. 2081-2084, 2008.

[42] W. Chung, S. H. Kim, and K. H. Shin, "A method for planar development of 3D surfaces in shoe pattern design," Journal of Mechanical Science and Technology, vol. 22, no. 5, pp. 1510-1519, 2008.

[43] M. J. Rupérez, C. Monserrat, and M. Alcañiz, "Simulation of the deformation of materials in shoe uppers in gait: Force distribution using finite elements," International Journal on Interactive Design and Manufacturing, vol. 2, no. 2, pp. 59-68, 2008.

[44] M. J. Rupérez, S. Alemany, C. Monserrat, J. Olaso, M. Alcañiz, and J. C. González, "A study of the viability of obtaining a generic animation of the foot while walking for the virtual testing of footwear using dorsal pressures," Journal of Biomechanics, vol. 42, pp. 2040-2046, 2009.

[45] M. Arbib, The Handbook of Brain Theory and Neural Networks. MIT Press, 2003.

[46] C. M. Bishop, Neural Networks for Pattern Recognition. Clarendon Press, 1995.

[47] R. O. Duda, P. E. Hart, and D. G. Stork, Pattern Classification. Wiley, 2001.

[48] B. D. Ripley, Pattern Recognition and Neural Networks. Cambridge University Press, 1996.

[49] E. Soria, J. D. Martín, and P. J. G. Lisboa, Clasical training methods. Metaheuristic procedures for training neural networks, Springer-Verlag, 2006. 
[50] P. J. G. Lisboa, "A review of evidence of health benefit from artificial neural networks in medical intervention," Neural Networks, vol. 15, no. 1, pp. 11$39,2002$.

[51] M. E. Brier, J. M. Zurada, and G. R. Aronoff, "Neural network predicted peak and trough gentamicin concentrations," Pharmaceutical research, vol. 12, no. 3, pp. 406-412, 1995.

[52] A. Nebot, F. E. Cellier, and D. A. Linkens, "Synthesis of an anaesthetic agent administration system using fuzzy inductive reasoning," Artificial Intelligence in Medicine, vol. 8, no. 2, pp. 147-166, 1996.

[53] X. S. Zhang, J. W. Huang, and R. J. Roy, "Modeling for neuromonitoring depth of anesthesia," Critical Reviews in Biomedical Engineering, vol. 30, no. 1-3, 2002.

[54] L. Leistritz, E. Kochs, M. Galicki, and H. Witte, "Prediction of movement following noxious stimulation during 1 minimum alveolar anesthetic concentration isoflurane/nitrous oxide anesthesia by means of middle latency auditory evoked responses," Clinical Neurophysiology, vol. 113, no. 6, pp. 930-935, 2002.

[55] M. E. Hernando, E. J. Gómez, F. D. Pozo, and R. Corcoy, "DIABNET: A qualitative model-based advisory system for therapy planning in gestational diabetes," Informatics for Health and Social Care, vol. 21, no. 4, pp. 359374, 1996.

[56] S. G. Mougiakakou and K. S. Nikita, "A neural network approach for insulin regime and dose adjustment in type 1 diabetes," Diabetes Technology 8 Therapeutics, vol. 2, no. 3, pp. 381-389, 2000.

[57] Z. Trajanoski, W. Regittnig, and P. Wach, "Simulation studies on neural predictive control of glucose using the subcutaneous route," Computer Methods and Programs in Biomedicine, vol. 56, no. 2, pp. 133-139, 1998.

[58] G. Hatzakis and C. Tsoukas, "Neural networks in the assessment of HIV immunopathology," In: Proceedings of the AMIA Symposium, p. 249, 2001.

[59] S. Sardari and D. Sardari, "Applications of artificial neural network in AIDS research and therapy," Current Pharmaceutical Design, vol. 8, no. 8, p. 659, 2002.

[60] J. D. Martín-Guerrero, G. Camps-Valls, E. Soria-Olivas, A. J. SerranoLópez, J. J. Pérez-Ruixo, and N. V. Jiménez-Torres, "Dosage individualization of erythropoietin using a profile-dependent support vector regression," 
IEEE Transactions on Biomedical Engineering, vol. 50, no. 10, pp. 1136$1142,2003$.

[61] J. D. Martín-Guerrero, E. S. Olivas, G. C. Valls, A. J. Serrano-López, J. J. Pérez-Ruixo, and N. V. J. Torres, "Use of neural networks for dosage individualisation of erythropoietin in patients with secondary anemia to chronic renal failure," Computers in Biology and Medicine, vol. 33, no. 4, pp. 361373, 2003.

[62] S. H. Holzreiter and M. E. Köhle, "Assessment of gait patterns using neural networks," Journal of Biomechanics, vol. 26, no. 6, p. 645, 1993.

[63] J. B. Bishop, M. Szpalski, S. K. Ananthraman, D. R. McIntyre, and M. H. Pope, "Classification of low back pain from dynamic motion characteristics using an artificial neural network," Spine, vol. 22, no. 24, pp. 2991-2298, 1997.

[64] J. G. Barton, A. Lees, P. Lisboa, and S. Attfield, "Visualisation of gait data with Kohonen self-organising neural maps," Gait \& Posture, vol. 24, no. 1, pp. 46-53, 2006.

[65] J. G. Barton, "Interpretation of gait data using Kohonen neural networks," Gait \& Posture, vol. 10, no. 1, pp. 85-86, 1999.

[66] J. G. Barton, P. Lisboa, and A. Lees, "Topological clustering of patients using a self organising neural map," Gait ES Posture, vol. 12, no. 1, p. 57, 2000 .

[67] J. G. Barton, P. Lisboa, A. Lees, and S. Attfield, "Gait quality assessment using self-organising artificial neural networks," Gait \& Posture, vol. 25, no. 3, pp. 347-379, 2007.

[68] J. G. Barton and A. Lees, "Comparison of shoe insole materials by neural network analysis," Medical and Biological Engineering and Computing, vol. 34, no. 6, pp. 453-459, 1996.

[69] J. M. Twomey, A. E. Smith, and M. S. Redfern, "A predictive model for slip resistance using artificial neural networks," IIE Transactions, vol. 27, no. 3, pp. 374-381, 1995.

[70] B. Kirk, M. Carré, S. Haake, and G. Manson, "Using neural networks to understand relationships in the traction of studded footwear on sports surfaces," Journal of Biomechanics, vol. 39, no. S1, p. S183, 2006. 
[71] M. J. Rupérez, J. D. Martín, C. Monserrat, and M. Alcañiz, "Neural networks for modeling the contact foot-shoe upper," Machine Learning Applications and Trends: Algorithms, Methods, and Techniques, vol. 2, no. 27, pp. 561-573, 2010.

[72] M. J. Rupérez, J. D. Martín-Guerrero, C. Monserrat, and M. Alcañiz, "Artificial neural networks for predicting dorsal pressures on the foot surface while walking," Expert System with Application. Submitted.

[73] M. J. Rupérez, J. D. Martín-Guerrero, C. Monserrat, and M. Alcañiz, "Modeling dorsal pressures on the foot surface using artificial neural networks," In: Proceedings of the XXII Congress of the International Society of Biomechanics, Cape Town, South Africa, 2009.

[74] B. K. Wong and Y. Selvi, "Neural network applications in Finance: A review and analysis of literature (1990-1996)," Information and Management, vol. 34, no. 3, pp. 129-139, 1998.

[75] B. Widrow, D. E. Rumelhart, and M. A. Lehr, "Neural networks: Applications in Industry, Business and Science," Communications of the ACM, vol. 37, no. 3, pp. 93-105, 1994.

[76] B. Curry and L. Moutinho, "Neural networks in marketing: Modelling consumer responses to advertising stimuli," European Journal of Marketing, vol. 27, no. 7, pp. 5-20, 1993.

[77] M. Plumbley, "Communications and neural networks: Theory and practice," EEE International Conference on Acoustics, Speech, and Signal Processing (ICASSP'97), vol. 1, p. 135, 1997.

[78] C. Fernández, E. Soria, J. D. Martín, and A. J. Serrano, "Neural networks for animal science applications: Two case studies," Expert Systems with Application, vol. 31, no. 2, pp. 444-450, 2006.

[79] J. J. Holewski, K. M. Moss, R. M. Stess, P. M. Graf, and C. Grunfeld, "Prevalence of foot pathology and lower extremity complications in a diabetic outpatient clinic," Journal of Rehabilitation Research and Development, vol. 26, no. 3, pp. 35-44, 1989.

[80] O. N. Onwuanyi, "Calcaneal spurs and plantar heel pad pain," The Foot, vol. 10, pp. 182-185, 2000.

[81] J. C. González, E. Alcántara, A. Bataller, and A. Gracía, "Physiological and subjective evaluation of footwear thermal response over time," In: Proceeding of Fifth Symposium on Footwear Biomechanics. Hennig, E., Stacoff, A., Gerber, H., (Eds.). Zürich, pp. 40-41, 2001. 
[82] O. Zienkiewicz and R. Taylor, El método de los elementos finitos, vol. 1. New York, NY, USA: McGraw-Hill, 1994.

[83] J. L. Batoz, "An explicit formulation for an efficient triangular platebending element," International Journal for Numerical Methods in Engineering, vol. 18, no. 7, pp. 1077-1089, 1982.

[84] J. L. Batoz, K. J. Bathe, and L. W. Ho, "A study of three-node triangular plate bending elements," International Journal for Numerical Methods in Engineering, vol. 15, no. 12, pp. 1771-1812, 1980.

[85] O. Zienkiewicz and R. Taylor, El método de los elementos finitos, vol. 2. New York, NY, USA: McGraw-Hill, 1994.

[86] P. G. Bergan and C. A. Felippa, "A triangular membrane element with rotational degrees of freedom," Computer Methods in Applied Mechanics and Engineering, vol. 50, pp. 25-69, 1985.

[87] T. Belytschko, W. K. Liu, and B. Moran, Nonlinear Finite elements for Continua and Structures. Wiley, 1998.

[88] R. Macneal and R. L. Harder, "A proposed standard set of problems to test finite element accuracy," Finite Element Analysis Design, vol. 1, pp. 3-20, 1985 .

[89] R. G. Mitton, "Mechanical properties of leather fibres," Journal of Society Leather Trades' Chemists, vol. 29, pp. 169-194, 1945.

[90] R. G. Mitton, "Tensile properties and their variability in chrome-tanned calfskins," Journal of Society Leather Trades' Chemists, vol. 32, pp. 310$323,1948$.

[91] M. Wilson, R. Browne, and S. Rose, "Low modulus, 'stretchy' upper materials," SATRA Bulletin, no. June, 1995.

[92] J. Olaso, J. C. González, S. Alemany, E. Medina, A. López, C. Martín, J. Prat, and C. Soler, "Study of the influence of fitting and walking condition in foot dorsal pressure," In proceedings of 8th Footwear Biomechanics Symposium. Frederick E. C., Yang, S.W., (Eds.). Taipei, Taiwan, pp. 4142, 2007.

[93] A. M. Habraken and S. Cescotto, "Contact between deformable solids: The fully coupled approach," Mathematical and Computer Modelling, vol. 28, no. 4-8, pp. 153-169, 2006. 
[94] D. Pantuso, K. J. Bathe, and P. A. Bouzinov, "A finite element procedure for the analysis of thermo-mechanical solids in contact," Computers $\&$ Structures, vol. 75, no. 6, pp. 551-573, 2000.

[95] P. Papadopoulus and J. M. Solberg, "A lagrange multiplier method for the finite element solution of frictionless contact problems," Mathematical and Computer Modelling, vol. 28, no. 12, pp. 373-384, 1998.

[96] J. M. Solberg and P. Papadopoulus, "A finite element method for contact/impact," Finite Elements in Analysis Design, vol. 30, no. 15, pp. 297311, 1998.

[97] P. Wriggers, Computational Contact Mechanics. Second ed. Springer, 2006.

[98] C. Jordan, C. J. Payton, and R. M. Bartlett, "Perceived comfort and pressure distribution in casual footwear," In Proceedings of the Fifth EMED User Meeting. Clinical Biomechanics, vol. 12, no. 3, p. 5, 1997.

[99] J. García-Hernández, S. Heras, J. Alfons, R. Paredes, B. Nácher, S. Alemany, E. Alcántara, and J. C. González, "The MORFO3D foot database," Lecture Notes in Computer Science, vol. 3523, pp. 658-665, 2005.

[100] B. Nácher, E. Alcántara, S. Alemany, J. García-Hernández, and A. Juan, "3D foot digitizing and its application to footwear fitting," In: Proceedings of 3D Modeling (Numerisation 3D), Paris, France, 2004.

[101] M. C. Carson, M. E. Harrington, N. Thompson, J. J. O'Connor, and T. N. Theologis, "Kinematic analysis of a multi-segment foot model for research and clinical applications: A repeatability analysis," Journal of Biomechanics, vol. 34, no. 10, pp. 1299-1307, 2001.

[102] B. A. MacWilliams, M. Cowley, and D. E. Nicholson, "Foot kinematics and kinetics during adolescent gait," Gait \& Posture, vol. 17, no. 3, pp. 214-224, 2003.

[103] F. Bookstein, Morphometric tools for landmark data. Cambridge University Press, 1991.

[104] J. Lin and D. R. Hayhurst, "Constitutive equations for multi-axial straining of leather under uni-axial stress," European Journal of Mechanics A/Solids, vol. 12 , no. 4, pp. 471-492, 1993.

[105] W. T. Roddy, Chemistry and Technology of Leathers. American Chemical Society Monograph 134, New York: Reinhold Publishing Corp, 1956. 
[106] J. R. Kanagy, "Significance of the results of some physical upper leather," Journal of American Leather Chemists Association, vol. 50, pp. 112-148, 1955.

[107] A. G. Marriot, Some mechanical properties of air dry leather impregnated with film forming polymers. PhD thesis, University of Surrey, UK, 1978.

[108] Y. Lanir and Y. Fung, "Two-dimensional mechanical properties of rabbit skin-1. Experimental results," Journal of Biomechanics, vol. 7, pp. 29-34, 1974

[109] Y. Lanir and Y. Fung, "Two-dimensional mechanical properties of rabbit skin-2. Experimental results," Journal of Biomechanics, vol. 7, pp. 171-182, 1974

[110] M. Maeser, Dynamic and nondestructive testing of leather. American Chemical Society Monograph 134, New York: Reinhold Publishing Corp, 1956.

[111] J. Lin, I. C. H. D. R. Hayhurst, and D. C. Reedman, "Modelling of the performance of leather in a uni-axial shoe-last simulator," Journal of Strain Analysis, vol. 27, no. 4, pp. 187-196, 1992.

[112] E. Giner and J. Albelda, Análisis y diseño de materiales compuestos. Universidad Politécnica de Valencia, 2001.

[113] S. Haykin, Neural networks: A comprehensive foundation (3rd Ed.). New York: Macmillan, 2008.

[114] S. Haykin, Adaptive Filter Theory. Prentice-Hall, 1996.

[115] R. Hecht-Nielsen, Neurocomputing. Addison-Wesley, 1989.

[116] G. Cybenko, Continuous valued neural network with two hidden layers are sufficient. Report, Department of Computer Science, Tufts University, Medford, USA, 1988.

[117] C. M. Bishop, Pattern Recognition and Machine Learning. Springer, Singapore, 2006.

[118] M. H. Hassoun, Fundamentals of Artificial Neural Networks. MIT Press, 1995.

[119] Y. LeCun, "Une procedure d'apprentissage pour reseau a seuil asymmetrique (a learning scheme for asymmetric threshold networks)," Proceedings of Cognitiva, vol. 85, pp. 599-604, 1985. 
[120] D. E. Rumelhart, G. E. Hinton, and R. J. Williams, "Learning representations by back-propagating errors," Nature, vol. 323, pp. 533-536, 1986.

[121] P. J. Werbos, Beyond regression: New tools for prediction and analysis in the behavioral sciences. PhD thesis, Harvard University, Cambridge, MA, USA, 1974.

[122] D. G. Luenberger, Linear and Nonlinear Programming. Addison-Wesley, 1984.

[123] G. B. Orr and K. R. Müller, eds., Neural Networks: Tricks of the Trade. Lecture Notes on Computer Science. Springer, 1998.

[124] D. Gorse, A. J. Shepherd, and J. G. Taylor, "The new ERA in supervised learning," Neural Networks, vol. 10, no. 2, pp. 343-352, 1997.

[125] R. Reed, "Pruning algorithm - A survey," IEEE Transactions on Neural Networks, vol. 4, no. 5, pp. 740-747, 1993.

[126] R. D. Reed and R. J. M. II, Neural Smithing, Supervised Learning in Feedforward Artificial Neural Networks. Cambridge, MA, Estados Unidos: The MIT Press, 1999.

[127] S. Haykin, Neural Networks and Learning Machines, 3rd Edition. Pearson Education, Upper Saddle River, NJ, USA., 2009. 


\section{Glossary}

Counter: a semi-rigid cup of leather forming the back of the shoe designed to fit around the heel.

Cloud creep: a kind of foamed material.

Dorsal pressures: pressures on the upper surface of the foot.

$\boldsymbol{E} \boldsymbol{V A}$. Ethylene Vinyl Acetate: a kind of foamed material used in footwear to construct different protecting parts.

Facia: is a connective tissue that surrounds muscles, groups of muscles, blood vessels, and nerves.

Gait: a person's manner of walking.

Hallux valgus: is an enlargement of bone or tissue around the joint at the base of the big toe (metatarsophalangeal joint). The big toe (hallux) may turn in toward the second toe (angulation), and the tissues surrounding the joint may be swollen and tender.

Heelstrike: the beginning of stance phase of a step.

Insole: a removable sole worn in a shoe for warmth or to improve the fit or the fixed inner sole of a boot or shoe. 
Insole conformity: similarity in form or type.

Last: a shoemaker's model for shaping or repairing a shoe or boot.

Malleolus: is the name for the bony prominence on each side of the ankle.

Metatarsal bones: the metatarsal bones are a group of five long bones in the forefoot analogous to the metacarpal bones of the hand. Lacking individual names, the metatarsal bones are numbered from the medial side (side of big toe): the first, second, third, fourth, and fifth metatarsal.

Microcell Puff and Microcell Puff Lite: kinds of foamed material used to construct plugs for the midsoles aimed at reducing the plantar pressure.

Midsole: the middle part of a sole.

Pad: the fleshy underpart of an animal's foot or of a human finger.

Plantar pressures: pressures on the foot sole.

Plastazote: polyethylene foam designed to be modeled at $275^{\circ} \mathrm{F}$ from a model or from the patient's foot directly.

Poron: a kind of foamed material used to construct plugs for the midsoles aimed at reducing the plantar pressure.

Pronation: is a rotational movement of the foot at the subtalar and talocalcaneonavicular joints. For the foot, pronation will cause the sole of the foot to face more laterally than when standing in the anatomical position.

Push off: the end of stance phase of a step.

Shankpiece: a steel piece used to improve the resistance of high or medium high-heeled shoes.

Shoe upper: is the upper part of the shoe, that is the part of the shoe 
that is not the sole.

Stud: a small projection fixed to the base of a shoe or boot to provide better grip. 
Multidisciplinary Techniques for the Simulation of the Contact between the Foot and the Shoe Upper in Gait: Virtual Reality, Computational Biomechanics, and Artificial Neural Networks.

María José Rupérez Moreno, July 2011 
